\title{
Deep venous obstruction
}

Citation for published version (APA):

van Vuuren, TMAJ. (2019). Deep venous obstruction: towards optimizing treatment strategies. [Doctoral Thesis, Maastricht University]. Maastricht University. https://doi.org/10.26481/dis.20190110tv

Document status and date:

Published: 01/01/2019

DOI:

10.26481/dis.20190110tv

Document Version:

Publisher's PDF, also known as Version of record

\section{Please check the document version of this publication:}

- A submitted manuscript is the version of the article upon submission and before peer-review. There can be important differences between the submitted version and the official published version of record.

People interested in the research are advised to contact the author for the final version of the publication, or visit the DOI to the publisher's website.

- The final author version and the galley proof are versions of the publication after peer review.

- The final published version features the final layout of the paper including the volume, issue and page numbers.

Link to publication

\footnotetext{
General rights rights.

- You may freely distribute the URL identifying the publication in the public portal. please follow below link for the End User Agreement:

www.umlib.nl/taverne-license

Take down policy

If you believe that this document breaches copyright please contact us at:

repository@maastrichtuniversity.nl

providing details and we will investigate your claim.
}

Copyright and moral rights for the publications made accessible in the public portal are retained by the authors and/or other copyright owners and it is a condition of accessing publications that users recognise and abide by the legal requirements associated with these

- Users may download and print one copy of any publication from the public portal for the purpose of private study or research.

- You may not further distribute the material or use it for any profit-making activity or commercial gain

If the publication is distributed under the terms of Article $25 \mathrm{fa}$ of the Dutch Copyright Act, indicated by the "Taverne" license above, 


\section{Deep venous obstruction:}

towards optimizing treatment strategies 
(C) Timme van Vuuren, Maastricht 2019, All rights reserved

Cover: Peter van Vuuren

Layout: Tiny Wouters

Production: ProefschiftMaken || www.proefschriftmaken.nl

ISBN: 978-94-6380-153-9 


\title{
Deep venous obstruction:
}

\section{towards optimizing treatment strategies}

\author{
PROEFSCHRIFT \\ ter verkrijging van de graad van doctor aan de Universiteit Maastricht, \\ op gezag van de Rector Magnificus, Prof. dr. Rianne M. Letschert, \\ volgens het besluit van het College van Decanen, \\ in het openbaar te verdedigen \\ op vrijdag 11 januari 2019 om 12.00 uur
}

door

Timme Maria Angela Johannes van Vuuren 


\section{Promotor}

Prof. dr. C.H.A. Wittens

\section{Copromotor}

Dr. R. de Graaf

\section{Beoordelingscommissie}

Prof. dr. P.M. Steijlen (voorzitter)

Prof. dr. M.W. de Haan

Dr. R.J.M.W. Rennenberg

Dr. A.M. Wiersema (Westfriesgasthuis)

Prof. dr. W. Wisselink (VU Medisch Centrum) 


\section{Contents}

$\begin{array}{lll}\text { Chapter } 1 & \text { General introduction } & 7\end{array}$

Chapter 2 Reconstruction of the femoro-ilio-caval outflow by 21 percutaneous and hybrid interventions in symptomatic deep venous obstruction

Chapter 3 Patency rates and clinical outcomes in a cohort of 200 patients treated with a dedicated venous stent

Chapter 4 Stent extension into a single inflow vessel is a valuable option after endophlebectomy

Chapter 5 Stent extension below the common femoral vein in extensive chronic iliofemoral venous obstruction

Chapter 6 Abdominal and pubic collateral veins as indicators of deep venous obstruction

Chapter 7 Venous stent patency may be affected by collateral vein lumen size

Chapter 8 Illusory angiographic signs of significant iliac vein compression in healthy volunteers

Chapter 9 The Self-Assessed Venous Severity questionnaire for monitoring symptoms of deep venous obstructions

Chapter 10 A randomized controlled trial comparing venous stenting with conservative treatment in patients with deep venous obstruction: research protocol

Nederlandse samenvatting

Valorisation addendum

List of publications

Dankwoord 



\section{Chapter 1}

Deep venous obstruction

Current outcomes and implications for improvement 



\section{General introduction}

Deep venous obstruction is relatively common in patients with chronic venous disease and is caused by either post-thrombotic vein damage, extraluminal vein compression or a combination of both. With a deep venous thrombosis (DVT) incidence of 1-2 per 1000 patients per year, a large medical and economic burden is created. ${ }^{1,2}$

As already mentioned in Virchow's triad, a DVT can occur due to a hypercoagulable state, vascular wall damage and venous stasis. ${ }^{3}$ For a long time, the hypercoagulable state and venous stasis have been encompassed in the conservative management of a DVT. The vein wall damage was initially not incorporated in the treatment strategy since, until a few years ago, valvar insufficiency was thought to be the singular contributing factor. ${ }^{1,4,5}$ With increasing in-depth knowledge of the pathophysiological mechanisms of a post-DVT state, it is now known that vein wall scarring and vein wall fibrosis occur because of incomplete recanalization. ${ }^{6-8}$ Therefore, venous outflow of the leg is compromised, and venous hypertension can occur. This is specifically an issue with intensified exercise. Especially in those patients with ilio femoral DVTs, this recanalization process is inadequate. These patients show a higher risk for developing a so-called post thrombotic syndrome (PTS) with incidences up to $50 \% .^{9-11}$

A second frequent cause of deep venous obstruction is extraluminal vein compression of which the iliac vein compression syndromes (IVCS) or non-thrombotic iliac vein compression syndromes are best known. ${ }^{12}$ Most frequently, the May-Thurner syndrome (MTS) is found. (Figure 1.1) This is an anatomical obstruction whereby the left common iliac vein is compressed between the fifth lumbar vertebral body and the right common iliac artery. ${ }^{13}$ Currently, it is believed that a compression of $>50 \%$ is significant thus indicating treatment when accompanied by clinical complaints. ${ }^{14-16}$ Although previous research presented a MTS prevalence of $24 \%$ in cadaver studies, the exact prevalence of symptomatic and clinically relevant MTS in the general population is still unknown. ${ }^{14,17-20}$

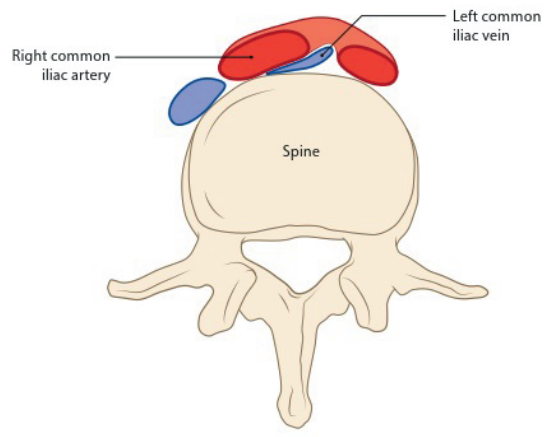

Figure 1.1 Example of May Thurner compression point: left common iliac vein is compressed between the fifth lumbar vertebral body and the right common iliac artery. 


\section{Clinical symptoms and signs}

Both the intraluminal and extraluminal vein obstruction can impede ambulatory venous outflow of the lower extremity and result in venous hypertension. These obstructions are associated with clinical signs and symptoms shared with other types of chronic venous disease, such as pain (but more specifically during ambulation), heaviness, and paresthesia. ${ }^{21}$ Moreover, clinical signs like varicose leg veins, hyperpigmentation, and eventually venous ulceration may develop over time.

All these venous complaints can be subdivided using several scores, with known advantages and disadvantages. One example is the Ginsberg score which was developed to find PTS after hip or knee arthroplasty. ${ }^{22}$ This score classified PTS as being present, when leg pain and leg swelling were present in addition to valvular incompetence. The main disadvantage of this score relies in the fact that it does not take vein occlusion into account.

A second clinical scoring item, the Brandjes score, was developed to investigate the effect of compression stockings in patients with a proximal DVT. ${ }^{23}$ The Brandjes score gives points to a number of subjective and objective criteria and classifies PTS as being present based on the score of two consecutive visits 3 months apart (Table 1.1). This score gives 4 points to the development of a venous ulcer which also brings the problem that, once a patient develops a venous ulcer, the score will remain high.

Table $1.1 \quad$ Brandjes scoring system.

\begin{tabular}{lclc}
\hline Subjective criteria & & Objective criteria & Score \\
\hline Symptoms & Score & Sign & 1 \\
\hline Spontaneous pain in calf & 1 & Calf circumference increased by $1 \mathrm{~cm}$ & 1 \\
Spontaneous pain in thigh & 1 & Ankle circumference increased by $1 \mathrm{~cm}$ & 1 \\
Pain in calf on standing/walking & 1 & Pigmentation & 1 \\
Pain in thigh on standing/walking & 1 & Venectasia & 1 \\
Edema of foot/calf & 1 & Newly formed varicosis & 1 \\
Heaviness of the leg & 1 & Phlebitis & 4 \\
Spontaneous pain and pain on walking/standing & 1 & Venous ulcer & \\
Impairment of daily activities & 1 & & \\
\hline
\end{tabular}

Next to abovementioned scoring systems, other classifications have been developed and have been used to diagnose and follow PTS symptoms. The Widmer score, initially developed for chronic venous insufficiency, is one example (Table 1.2.) The Widmer score relies purely on clinical signs which brings back the main disadvantage of it; as clinical signs merely change over time, this score is unable to follow results of PTS treatment. $^{24}$ 
Table 1.2 The Widmer scoring system.

\begin{tabular}{ll}
\hline Stage & Symptoms \\
\hline 1 & Ankle flare \\
& Subclinical edema \\
2 & Edema \\
& Pigmentation \\
& Lipodermatosclerosis \\
& White skin atrophy \\
& Leg ulcer \\
& Leg ulcer in the past \\
\hline
\end{tabular}

The CEAP classification, developed to score chronic venous disease, scores clinical signs according to the highest $\mathrm{C}$ score (Table 1.3). ${ }^{25}$ Unfortunately, this classification is inadequate, as it does not include the patients' complaints. Moreover, it is almost impossible to longitudinally follow changes in the $\mathrm{C}$ score since most skin changes merely change over time. Next to the CEAP classification, the Venous Clinical Severity Score (VCSS) is a validated scoring system, widely used to score patients' complaints and clinical signs. In this more dynamic score, a reflection of the patients' perception is taken into account (Table 1.4). ${ }^{26}$ Unfortunately, this comprises only 1 item, representing a maximum of 3 out of 30 points, or in other words, $10 \%$ of the total score. Consequently, this hardly ever influences the outcomes in a significant way.

Table 1.3 CEAP classification.

\begin{tabular}{ll}
\hline Class & Signs \\
\hline 0 & No visible or palpable signs of venous disease \\
1 & Telangiectases, reticular veins, malleolar flare \\
2 & Varicose veins \\
3 & Edema without skin changes \\
4 & Skin changes ascribed to venous disease (pigmentation, venous eczema, \\
& lipodermatosclerosis) \\
6 & Skin changes as above with healed ulceration \\
6 & Skin changes as above with active ulceration \\
\hline
\end{tabular}

For the post thrombotic syndrome, the Villalta scale was created for scoring clinical signs and symptoms uniformly and longitudinally (Table 1.5). ${ }^{27,28}$ Due to the lack of a gold standard diagnostic test for PTS, both clinical signs and patients' symptoms were incorporated in this classification. The main advantage of this scale is displayed by the fact that it includes the patients' experience. In addition, it was shown in previous research that Villalta scores correlate well with patient-perceived quality of life. ${ }^{1}$ 
Table 1.4 Venous clinical severity score.

\begin{tabular}{|c|c|c|c|c|}
\hline Descriptor & Score 0 & Score 1 & Score 2 & Score 3 \\
\hline Pain & None & $\begin{array}{l}\text { Occasional, not } \\
\text { restricting activity }\end{array}$ & $\begin{array}{l}\text { Daily, moderate, } \\
\text { limitation of activity }\end{array}$ & $\begin{array}{l}\text { Daily, severe, } \\
\text { limitation of } \\
\text { activities }\end{array}$ \\
\hline $\begin{array}{l}\text { Varicose veins (> } 4 \mathrm{~mm} \\
\text { diameter) }\end{array}$ & None & Few, scattered & $\begin{array}{l}\text { Multiple, GS } \\
\text { confined to calf or } \\
\text { thigh }\end{array}$ & $\begin{array}{l}\text { Extensive, calf } \\
\text { and thigh GS and } \\
\quad \text { LS }\end{array}$ \\
\hline Venous edema & None & $\begin{array}{l}\text { Evening ankle } \\
\text { edema }\end{array}$ & $\begin{array}{l}\text { Afternoon edema, } \\
\text { above ankle but } \\
\text { below knee }\end{array}$ & $\begin{array}{l}\text { Morning edema } \\
\text { above ankle and } \\
\text { knee }\end{array}$ \\
\hline Skin pigmentation & None & $\begin{array}{l}\text { Diffuse, limited in } \\
\text { area and old }\end{array}$ & $\begin{array}{l}\text { Diffuse, over most of } \\
\text { lower third or recent } \\
\text { pigmentation }\end{array}$ & $\begin{array}{l}\text { Wider } \\
\text { distributed, } \\
\text { above lower } \\
\text { third }\end{array}$ \\
\hline Inflammation & None & $\begin{array}{l}\text { Mild cellulitis, } \\
\text { marginal area } \\
\text { around ulcer }\end{array}$ & $\begin{array}{l}\text { Moderate cellulitis, } \\
\text { most of lower third }\end{array}$ & $\begin{array}{l}\text { Severe cellulitis, } \\
\text { above lower } \\
\text { third }\end{array}$ \\
\hline Induration & None & $\begin{array}{l}\text { Focal, } \\
\text { circummalleolar } \\
\qquad(<5 \mathrm{~cm})\end{array}$ & $\begin{array}{l}\text { Medial or lateral, } \\
\text { less than lower third } \\
\text { of leg }\end{array}$ & $\begin{array}{l}\text { Entire lower third } \\
\text { of leg or more }\end{array}$ \\
\hline No. Of active ulcers & 0 & 1 & 2 & 3 \\
\hline $\begin{array}{l}\text { Duration of active } \\
\text { ulceration in months }\end{array}$ & None & $<3$ & $3-12$ & $\begin{array}{l}\text { Not healed after } \\
12\end{array}$ \\
\hline $\begin{array}{l}\text { Diameter of largest ulcer in } \\
\mathrm{cm}\end{array}$ & None & $<2$ & $2-6$ & $>6$ \\
\hline $\begin{array}{l}\text { Use of compression } \\
\text { stockings }\end{array}$ & $\begin{array}{l}\text { Not used or non- } \\
\text { compliant }\end{array}$ & Intermittent use & Most days & Full compliance \\
\hline
\end{tabular}

GS: greater saphenous vein, LS: lesser saphenous vein

Table $1.5 \quad$ Villalta PTS score.

\begin{tabular}{|c|c|c|c|c|}
\hline Symptoms/ clinical signs & None & Mild & Moderate & Severe \\
\hline \multicolumn{5}{|l|}{ Symptoms } \\
\hline Pain & 0 points & 1 point & 2 points & 3 points \\
\hline Cramps & 0 points & 1 point & 2 points & 3 points \\
\hline Heaviness & 0 points & 1 point & 2 points & 3 points \\
\hline Paresthesia & 0 points & 1 point & 2 points & 3 points \\
\hline Pruritis & 0 points & 1 point & 2 points & 3 points \\
\hline \multicolumn{5}{|l|}{ Clinical signs } \\
\hline Pretibial edema & 0 points & 1 point & 2 points & 3 points \\
\hline Skin induration & 0 points & 1 point & 2 points & 3 points \\
\hline Hyperpigmentation & 0 points & 1 point & 2 points & 3 points \\
\hline Redness & 0 points & 1 point & 2 points & 3 points \\
\hline Venous ectasia & 0 points & 1 point & 2 points & 3 points \\
\hline Pain on calf compression & 0 points & 1 point & 2 points & 3 points \\
\hline
\end{tabular}




\section{Treatment of deep venous obstructions}

In the past, the only possible treatment option for PTS was conservative management, including the use of compression stockings, anticoagulation and an advice to walk as much as possible. Later, this was substituted by open bypass surgery in selected, severe post thrombotic cases. The high morbidity and moderate patency rates (34$88 \%$ ) caused by compression of the bypass or large hematomas called for new minimal invasive therapies. ${ }^{29,30}$

In order to reduce clinical symptoms with complication numbers as low as possible, several treatment options have been developed over the last decade. The introduction of duplex ultrasound, dedicated magnetic resonance venography protocols and phlebography's enabled this development of minimally invasive endovenous therapies. The evaluation of the deep and superficial venous system simplified, which in turn created opportunities to plan treatment more accurately than ever before. This rapid evolution in imaging techniques made possible, a superior evaluation of post thrombotic changes, like scarification, wall thickening, and irregularities as well as the extent of disease. ${ }^{31-34}$ Moreover, the collateral venous flow, as an indication of deep venous obstruction, can be examined. ${ }^{16}$

In post thrombotic obstructions, the interventional treatment is preferably performed under sedation or general anesthesia, due to the pain a patient can experience when dilating the vein with a balloon. Also, the procedure may take up to several hours, as the recanalization of the occluded or stenotic fibrotic tissue can be challenging. ${ }^{35}$ Venous access is derived with ultrasound guidance to prevent arterial cannulation or cannulation of collateral veins. With the patient in a supine position, a sheath is introduced into the femoral vein, through which a contrast agent can be administered. This contrast agent provides an overview image of the post thrombotic vessels, thereby also guiding the recanalization procedure. During this process, the treating physicians should carefully evaluate whether the correct pathway is recanalized, as patients with PTS often present with many collateral routes. It is therefore important to evaluate the recanalization course in both anterior-posterior and lateral planes. Subsequently, ballooning, stent sizing and stent deployment can be performed. Specific attention is given to completely cover the full diseased vein tract. ${ }^{36}$ Irrespective of the deployed stent type, balloon expendable or self-expendable stents, ballooning is performed after stent deployment in order to ensure optimal stent positioning and configuration (Figure 1.2). ${ }^{37,38}$ This endovenous PTA and stenting technique has been performed for several years and several studies have shown primary patency rates between $32 \%$ and $98.7 \%$ and secondary patency rates of $66 \%$ to $96 \% .^{39-41}$ with clinical improvement, illustrated by decreased Villalta scales and VCSS scores. 

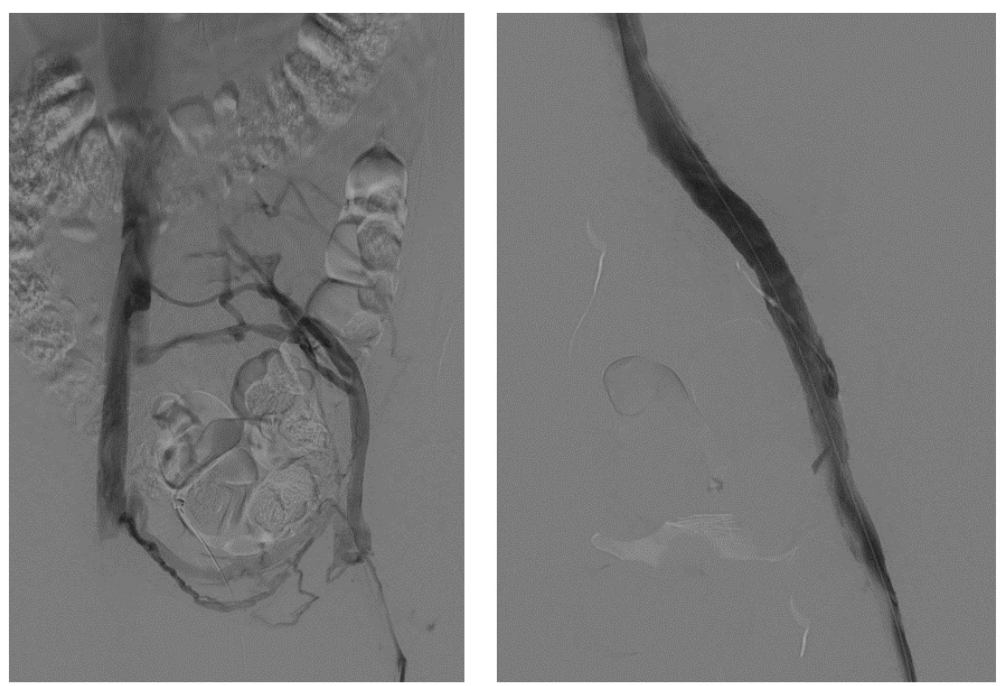

Figure 1.2 Left: Venography image of occluded left common iliac vein with numerous collaterals Right: Venography image after stent deployment showing normal venous outflow in left common iliac vein.

As inflow into stents seems to play a major role in sustaining stent patency, abovementioned percutaneous procedures are not advised to patients with extensive post thrombotic obstructions with involvement of major inflow veins. In cases were venous changes extend below the sapheno-femoral junction (SFJ), hybrid procedures including an endophlebectomy and arterio venous fistula (AVF) have been described in several small series to provide better inflow (Figure 1.3). ${ }^{42-44}$ In these cases, recanalization is performed first. Second, the common femoral vein is incised and as much intraluminal scar tissue as possible is removed. Afterwards, the vein is closed with or without a bovine patch, depending on the remaining vein lumen diameter. Next, an arteriovenous fistula is created between the common femoral vein and common femoral artery in order to prevent the thrombotic effects of the current desobstructed vein. At last, stents will be deployed in the post thrombotic vein tract and post-delivery dilated to the maximum diameter. 


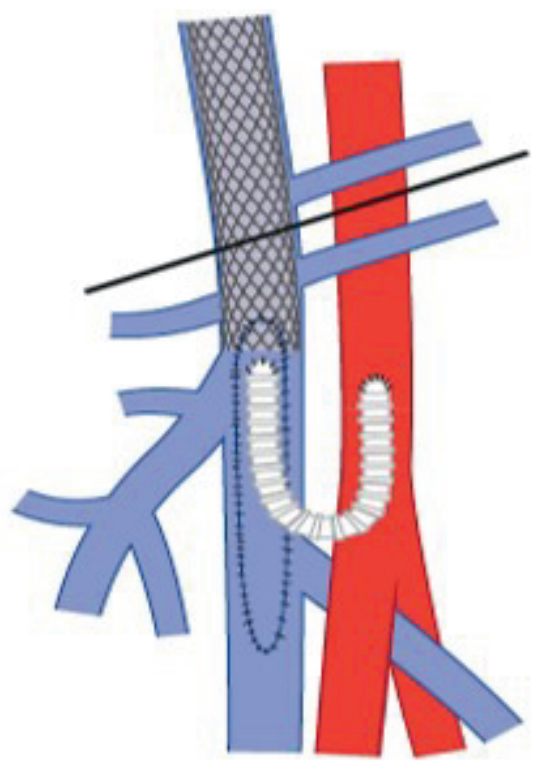

Figure 1.3 Arterio-venous fistula between common femoral vein and common femoral artery in a loop like fashion.

\section{Venous compression syndromes}

The second component of deep venous obstruction includes the iliac vein compression syndromes. The determined outflow obstruction caused by this compression can be treated similar to deep venous post thrombotic obstructions. About $30 \%$ of cases show some intraluminal spurs which also require recanalization before PTA. ${ }^{13}$ The most important issue to focus on, is correct stent placement in the common iliac vein. There should be a balance between solid coverage of the ipsilateral compression part without compromising the contralateral outflow.

Primary patency rates between $92 \%$ and $96 \%$ and secondary patency rates between $98 \%$ and $99 \%$ have been described with clinical improvement in several retrospective cohort studies. $^{40,41}$

\section{Points of improvement}

Although favorable outcomes and patency rates have been found, no clear factors that contribute to patency loss have been identified. Most current clinical evidence is 
based on retrospective, single-center studies though large prospective randomized trials are lacking.

Also, the incidence of (clinically relevant) iliac vein compression syndromes and more specifically MTS, in the general population is unclear. it is still unclear when exactly venous lumen reductions become significant enough to give rise to symptoms and thus when these should be treated.

Moreover, there is still debate regarding the classification of PTS. Currently, the Villalta scale is used a gold standard to diagnose PTS. When gaining more in-depth knowledge in PTS follow up, it was noticed that a large group of patients presents with low or moderate Villalta scores but debilitating complaints in daily life. As a result, venous claudication was introduced and scored for all PTS patients presenting at our outpatient clinic. Besides, as already suggesting in previous literature future research should focus on clinical scoring in relation to a patients' quality of life.

\section{Aims and outline of this thesis}

This thesis evaluates the outcomes of patients treated in the Maastricht University Medical Center and Klinikum Aachen over the past decade. We then identified variables that could influence and improve outcomes, specifically improve stent patency. This thesis investigates both the technical and clinical optimization methods and focused on pre-operative patient selection.

Chapter 11 summarizes the main outcomes and issues described in this book. 


\section{References}

1. Kahn SR, Shbaklo H, Lamping DL, Holcroft CA, Shrier I, Miron MJ, Roussin A, Desmarais S, Joyal F, Kassis J, Solymoss S, Desjardins L, Johri M, Ginsberg JS. Determinants of health-related quality of life during the 2 years following deep vein thrombosis. J Thromb Haemosts. 2008;6:1105-12.

2. Guanella R, Ducruet T, Johri M, Miron MJ, Roussin A, Desmarais S, Joyal F, Kassis J, Solymoss S, Ginsberg JS, Lamping DL, Shrier I, Kahn SR. Economic burden and cost determinants of deep vein thrombosis during 2 years following diagnosis: a prospective evaluation. J Thromb Haemost. 2011;9:2397-405.

3. Aird WC. Vascular bed-specific thrombosis. J Thromb Haemost. 2007;5 Suppl 1:283-91.

4. Mohr DN, Silverstein MD, Heit JA, Petterson TM, O'Fallon WM, Melton LJ. The venous stasis syndrome after deep venous thrombosis or pulmonary embolism: a population-based study. Mayo Clin Proc. 2000;75:1249-56.

5. Prandoni P, Villalta S, Bagatella P, Rossi L, Marchiori A, Piccioli A, Bernardi E, Girolami B, Simioni P, Girolami A. The clinical course of deep-vein thrombosis. Prospective long-term follow-up of 528 symptomatic patients. Haematologica. 1997;82:423-8.

6. Neglen P, Thrasher TL, Raju S. Venous outflow obstruction: An underestimated contributor to chronic venous disease. J Vasc Surg. 2003;38:879-85.

7. Kurstjens RL, de Wolf MA, Konijn HW, Toonder IM, Nelemans PJ, de Graaf R, Wittens CH. Intravenous pressure changes in patients with postthrombotic deep venous obstruction: results using a treadmill stress test. J Thromb Haemost. 2016;14:1163-70.

8. Comerota AJ, Oostra C, Fayad Z, Gunning W, Henke P, Luke C, Lynn A, Lurie F. A histological and functional description of the tissue causing chronic postthrombotic venous obstruction. Thromb Res. 2015;135:882-7.

9. Kahn SR, Shrier I, Julian JA, Ducruet T, Arsenault L, Miron MJ, Roussin A, Desmarais S, Joyal F, Kassis J, Solymoss S, Desjardins L, Lamping DL, Johri M, Ginsberg JS. Determinants and time course of the postthrombotic syndrome after acute deep venous thrombosis. Ann Intern Med. 2008;149:698-707.

10. Subbiah R, Aggarwal V, Zhao H, Kolluri R, Chatterjee S, Bashir R. Effect of compression stockings on post thrombotic syndrome in patients with deep vein thrombosis: a meta-analysis of randomised controlled trials. Lancet Haematol. 2016;3:e293-300.

11. Vedantham S, Goldhaber SZ, Kahn SR, Julian J, Magnuson E, Jaff MR, Murphy TP, Cohen DJ, Comerota AJ, Gornik HL, Razavi MK, Lewis L, Kearon C. Rationale and design of the ATTRACT Study: a multicenter randomized trial to evaluate pharmacomechanical catheter-directed thrombolysis for the prevention of postthrombotic syndrome in patients with proximal deep vein thrombosis. Am Heart J. 2013;165:523-30.e3.

12. May R, Thurner J. The cause of the predominantly sinistral occurrence of thrombosis of the pelvic veins. Angiology. 1957;8:419-27.

13. Cockett FB, Thomas ML. The iliac compression syndrome. Br J Surg. 1965;52:816-21.

14. Nordstrom M, Lindblad B, Bergqvist D, Kjellstrom T. A prospective study of the incidence of deep-vein thrombosis within a defined urban population. J Intern Med. 1992;232:155-60.

15. Cheng L, Zhao H, Zhang FX. Iliac Vein Compression Syndrome in an Asymptomatic Patient Population: A Prospective Study. Chinese Med J. 2017;130:1269-75.

16. Kurstjens RL, de Wolf MA, van Laanen JH, de Haan MW, Wittens $\mathrm{CH}$, de Graaf R. Hemodynamic significance of collateral blood flow in chronic venous obstruction. Phlebology. 2015;30:27-34.

17. Raju S, Neglen P. High prevalence of nonthrombotic iliac vein lesions in chronic venous disease: a permissive role in pathogenicity. J Vasc Surg. 2006;44:136-43; discussion 44.

18. Brinegar KN, Sheth RA, Khademhosseini A, Bautista J, Oklu R. Iliac vein compression syndrome: Clinical, imaging and pathologic findings. World J Radiol. 2015;7:375-81.

19. Kibbe MR, Ujiki M, Goodwin AL, Eskandari M, Yao J, Matsumura J. Iliac vein compression in an asymptomatic patient population. J Vasc Surg. 2004;39:937-43. 
20. Oguzkurt L, Ozkan U, Ulusan S, Koc Z, Tercan F. Compression of the left common iliac vein in asymptomatic subjects and patients with left iliofemoral deep vein thrombosis. J Vasc Intervent Radiol. 2008;19:366-70; quiz 71.

21. Wittens C, Davies AH, Baekgaard N, Broholm R, Cavezzi A, Chastanet S, de Wolf M, Eggen C, Giannoukas A, Gohel M, Kakkos S, Lawson J, Noppeney T, Onida S, Pittaluga P, Thomis S, Toonder I, Vuylsteke M, Kolh P, de Borst GJ, Chakfe N, Debus S, Hinchliffe R, Koncar I, Lindholt J, de Ceniga MV, Vermassen F, Verzini F, De Maeseneer MG, Blomgren L, Hartung O, Kalodiki E, Korten E, Lugli M, Naylor R, Nicolini P, Rosales A. Editor's Choice - Management of Chronic Venous Disease: Clinical Practice Guidelines of the European Society for Vascular Surgery (ESVS). Eur J Vasc Endovasc Surg. 2015;49:678-737.

22. Ginsberg JS, Turkstra F, Buller HR, MacKinnon B, Magier D, Hirsh J. Postthrombotic syndrome after hip or knee arthroplasty: a cross-sectional study. Arch Intern Med. 2000;160:669-72.

23. Brandjes DP, Buller HR, Heijboer H, Huisman MV, de Rijk M, Jagt H, ten Cate JW. Randomised trial of effect of compression stockings in patients with symptomatic proximal-vein thrombosis. Lancet (London, England). 1997;349:759-62.

24. Kolbach DN, Sandbrink MW, Neumann HA, Prins MH. Compression therapy for treating stage I and II (Widmer) post-thrombotic syndrome. Cochrane Database Syst Rev. 2003:Cd004177.

25. Rabe E, Pannier F. Clinical, aetiological, anatomical and pathological classification (CEAP): gold standard and limits. Phlebology. 2012;27 Suppl 1:114-8.

26. Marston WA, Vasquez MA, Lurie F, Wakefield TW, Rabe E, Shortell CK, Lohr JM, Passman MA, McLafferty RB. Multicenter assessment of the repeatability and reproducibility of the revised Venous Clinical Severity Score (rVCSS). J Vasc Surg Venous Lymphat Disord. 2013;1:219-24.

27. Wik HS, Enden TR, Ghanima W, Engeseth M, Kahn SR, Sandset PM. Diagnostic scales for the postthrombotic syndrome. Thromb Res. 2017.

28. Rabinovich A, Kahn SR. The postthrombotic syndrome: current evidence and future challenges. J Thromb Haemost. 2017;15:230-41.

29. AbuRahma AF, Robinson PA, Boland JP. Clinical, hemodynamic, and anatomic predictors of long-term outcome of lower extremity venovenous bypasses. J Vasc Surg. 1991;14:635-44.

30. Palma EC, Esperon R. Vein transplants and grafts in the surgical treatment of the postphlebitic syndrome. J Cardiovasc Surg. 1960;1:94-107.

31. Labropoulos N, Borge M, Pierce K, Pappas PJ. Criteria for defining significant central vein stenosis with duplex ultrasound. J Vasc Surg. 2007;46:101-7.

32. Arnoldussen C, Lam Y, Ito N, Winkens B, Kooi ME, Wittens CHA, Wildberger JE. Gadobutrol versus gadofosveset-trisodium in MR venography of the lower extremities. Eur Radiol. 2017;27:4986-94.

33. Arnoldussen C, Strijkers R, Lambregts D, Lahaye M, de Graaf R, Wittens C. Feasibility of identifying deep vein thrombosis characteristics with contrast enhanced MR-Venography. Phlebology. 2014;29:119-24.

34. Arnoldussen CW, Kurstjens R, de Wolf MA, de Graaf R, Das M, Wittens $\mathrm{CH}$. Assessment of postoperative and postintervention remodeling with dynamic $C T$ venography after treatment of chronic venous obstructions: important observations. J Vasc Surg Venous Lymphat Disord. 2014;2:106-7.

35. Mahnken AH, Thomson K, de Haan M, O'Sullivan GJ. CIRSE standards of practice guidelines on iliocaval stenting. Cardiovasc Intervent Radiol. 2014;37:889-97.

36. Jalaie H, Schleimer K, Barbati ME, Gombert A, Grommes J, de Wolf MA, de Graaf R, Wittens $\mathrm{CH}$. Interventional treatment of postthrombotic syndrome. Gefasschirurgie. 2016;21:37-44.

37. de Graaf R, de Wolf M, Sailer AM, van Laanen J, Wittens C, Jalaie H. Iliocaval Confluence Stenting for Chronic Venous Obstructions. Cardiovasc Intervent Radiol. 2015;38:1198-204.

38. de Graaf R, Arnoldussen C, Wittens $\mathrm{CH}$. Stenting for chronic venous obstructions a new era. Phlebology. 2013;28 Suppl 1:117-22.

39. Seager MJ, Busuttil A, Dharmarajah B, Davies AH. Editor's Choice-- A Systematic Review of Endovenous Stenting in Chronic Venous Disease Secondary to Iliac Vein Obstruction. Eur J Vasc Endovasc Surg. 2016;51:100-20. 
40. Wen-da W, Yu Z, Yue-Xin C. Stenting for chronic obstructive venous disease: A current comprehensive meta-analysis and systematic review. Phlebology. 2016;31:376-89.

41. Razavi MK, Jaff MR, Miller LE. Safety and effectiveness of stent placement for iliofemoral venous outflow obstruction: systematic review and meta-analysis. Circ Cardiovasc Interv. 2015;8:e002772.

42. Comerota AJ, Grewal NK, Thakur S, Assi Z. Endovenectomy of the common femoral vein and intraoperative iliac vein recanalization for chronic iliofemoral venous occlusion. J Vasc Surg. 2010;52:243-7.

43. Vogel D, Comerota AJ, Al-Jabouri M, Assi Zl. Common femoral endovenectomy with iliocaval endoluminal recanalization improves symptoms and quality of life in patients with postthrombotic iliofemoral obstruction. J Vasc Surg. 2012;55:129-35.

44. Puggioni A, Kistner RL, Eklof B, Lurie F. Surgical disobliteration of postthrombotic deep veins-endophlebectomy--is feasible. J Vasc Surg. 2004;39:1048-52. 



\section{Chapter 2}

Reconstruction of the femoro-ilio-caval outflow by percutaneous and hybrid interventions in symptomatic deep venous obstruction 


\section{Abstract}

\section{Objectives/background}

Deep venous obstruction is relatively prevalent in patients with chronic venous disease. Endovascular treatments and hybrid interventions can be used to relieve venous outflow obstructions. This paper assesses mid-term clinical outcomes and patency rates in a large cohort after percutaneous and hybrid interventions.

\section{Methods}

This was a prospectively analysed cohort study. Patients with symptomatic deep venous obstruction who presented at a tertiary referral hospital were divided into three groups: patients who underwent percutaneous stenting for non-thrombotic iliac vein compression syndrome (IVCS group); patients with postthrombotic syndrome (PTS) treated by percutaneous stent placement (P-PTS group); and PTS patients with obstruction involving the veins below the saphenofemoral junction in which a hybrid procedure was performed, combining stenting with open surgical disobliteration (H-PTS group). Patency rates, complications, and clinical outcomes were analysed.

\section{Results}

A total of 425 lower extremities in 369 patients were treated. At 60 months, primary patency, assisted primary patency, and secondary patency rates were $90 \%, 100 \%$, and $100 \%$ for IVCS, and $64 \%, 81 \%$, and $89 \%$ for the P-PTS group, respectively. The H-PTS group, showed patency rates of $37 \%, 62 \%$, and $72 \%$, respectively, at 36 months. Venous claudication subsided in $90 \%$, $82 \%$, and $83 \%$, respectively. At the 24 month follow-up, mean Venous Clinical Severity Score decreased for all patients and improvement in Villalta score was seen in postthrombotic patients. The number of complications was related to the extent of deep venous obstruction in which patients in the H-PTS group showed the highest complication rates (81\%) and reinterventions (59\%).

\section{Conclusion}

Percutaneous stent placement to treat non-thrombotic iliac vein lesions, and post-thrombotic iliofemoral obstructions are safe, effective, and showed patency rates comparable with previous research. Patients with advanced disease needing a hybrid procedure showed a lower patency rate and more complications. However, when successful, the clinical outcome was favourable at mid-term follow-up and the procedure may be offered to selected patients. 


\section{Introduction}

Minimally invasive endoluminal interventions have revolutionised many facets of modern medicine. Since the 1990s great advances have been made in the treatment of iliac and vena cava obstructions where endovascular treatment options frequently surpass conservative therapy and open surgery ${ }^{1-5}$

Caval and ilio-femoral venous obstructive disease is common in patients with chronic venous insufficiency and is caused by either post-thrombotic vein damage, extraluminal vein compression, or a combination of both. This post-thrombotic vein damage with vein wall fibrosis, intraluminal scarification, and valvular damage can result after a deep venous thrombosis (DVT), presenting as the so called postthrombotic syndrome (PTS). ${ }^{6,7}$ Clinically, this is associated with the same symptoms and clinical signs as other types of chronic venous disease, for example pain (especially during ambulation), oedema, and venous ulceration.

As mentioned, the second most common cause of chronic venous obstruction is related to extraluminal vein compression of which iliac vein compression syndromes (IVCS) are the best known. IVCS consist of symptoms associated with chronic venous disease due to compression of the iliac vein by an extraluminal structure (usually an overlying artery). ${ }^{8}$ Additionally, there can be some degree of spurs or webs due to chronic local vein wall irritation in about $30 \%$ of cases. ${ }^{9,10}$ In this subgroup, a smooth vessel wall and focal indentation will be seen during recanalisation, indicating compression without post thrombotic vein damage. ${ }^{11}$ Both the post-thrombotic and IVCS obstruction impede ambulatory venous outflow of the lower extremity and lead to venous hypertension. Venous outflow of the leg seems most dependent on the iliac tract, which explains the significantly higher incidence of PTS after an ilio-femoral DVT when compared with calf level DVTs. ${ }^{6,12}$ PTA and stenting of iliac lesions have been shown to improve outflow of affected legs. ${ }^{1-4}$ Moreover, stenting is performed and has been proven to be more effective in patients with a common femoral vein (CFV), external iliac vein, common iliac vein (CIV), or inferior vena cava (IVC) obstruction. ${ }^{2-4}$ Because stenting distal to the CFV has a higher risk of early failure due to low flow, this is generally not offered and is avoided. ${ }^{1,13-15}$ In addition, there is currently no evidence suggesting any clinical benefit from stenting below the common femoral confluence, which is where the deep femoral vein (DFV) and femoral vein (FV) merge into the CFV.

However, open surgical disobliteration, may be a solution for selected patients with post-thrombotic changes at and below the CFV. ${ }^{16-18}$

The goal of this study is to report on the experience, clinical outcome, and stent patency in patients treated for IVCS and post-thrombotic femoral, iliac, and caval obstructive lesions by percutaneous and hybrid techniques. 


\section{Methods}

In this cohort study data were collected prospectively and analysed retrospectively. All patients eligible for treatment at the authors' tertiary referral care centre (vascular surgery department) were included. Between September 2009 and January 2016, 369 consecutive patients were treated. All patients showed signs of chronic caval, iliac, or femoral vein obstruction on diagnostic imaging and demonstrated symptoms of venous hypertension like pain interfering with daily activities, and/or clinical signs classified according to the CEAP classification as C3 (edema) or C4a-C6 (skin changes). ${ }^{19}$ Additionally, venous claudication was a symptom indicating treatment and was defined as the onset or worsening of pain during routine exercise, which subsides during rest, especially while sitting or lying down. ${ }^{14}$

Patients who received stents after catheter directed thrombolysis for an acute DVT were excluded from this study. Non-fully grown adolescents (bone age analysed if applicable) with a DVT <1 year previously, and patients with a life expectancy of $<1$ year were not eligible for treatment. Also, patients with an occlusion extending far into the FV and DFV were not eligible for treatment as it is impossible to re-establish the inflow to the stent. Furthermore, treatment was not offered to those patients who were intolerant of anticoagulant therapy since this was obligatory after the intervention.

Patients had magnetic resonance venography (MRV) and duplex ultrasonography (DUS) imaging at baseline, and conventional multiplanar venography during intervention.

IVCS was defined as clinical complaints related to a $>50 \%$ lumen diameter reduction on DUS or MRV in combination with compression and a collateral network on venography. Intraluminal synechiae outside the compressed segment, with formation of collaterals, was considered a sign of post-thrombotic obstruction.

On the basis of findings, three groups were created in which interventions were performed. The IVCS patients with non-thrombotic syndrome had percutaneous stent placement alone. Percutaneous stent placement (P-PTS group) patients, had a percutaneous stent placement when obstruction was limited to segments central to the saphenofemoral junction (SFJ) in the CFV or when a trabeculation free landing zone in the CFV between the SFJ and the DFV and FV confluence was identified. The inflow from the FV, DFV, and/or collaterals was minimally impaired.

The third group (H-PTS) consisted of patients with PTS and obstruction below the SFJ, especially when the CFV was occluded or when there was a risk that stent placement could displace intraluminal tissue and obstruct FV or DFV inflow. Also, when the orifices of the FV and/or the DFV were occluded patients were included in the H-PTS group. 
Furthermore, endophlebectomy and arteriovenous fistulae (AVF) were performed as a second stage in some patients treated in the P-PTS group with stent thrombosis and clinical complaints interfering with daily activities.

Clinical scores, stent patency rates, complication rates, and number of reinterventions are presented per leg for all three groups.

\section{Clinical assessment before intervention}

At baseline, CEAP classification and the Venous Clinical Severity Score (VCSS) were scored for all patients. PTS was diagnosed using the Villalta score. With increasing clinical experience, the importance of venous claudication was recognised and a separate scoring for this item was added to the baseline and follow-up data collection. For this reason, not all venous claudication scores were present at baseline. The number of entered data is described in the text. Consequently, scores were inconsistently entered into a digital database at the various follow-up visits and at the discretion of the treating physician.

\section{DUS}

DUS (MyLab Alpha, Esaote, Genoa, Italy; ProSound Alpha 7 Premier machine, Hitachi Aloka, Tokyo, Japan) at baseline included imaging of the IVC from the level of the liver downward, the renal and gonadal veins, the iliac veins, and any apparent collateral network in the supine position using a convex probe (frequency range 1-8 $\mathrm{MHz}$ ). Special attention was given to any clues of post-thrombotic damage or IVCS, that is, venous stenosis, external vein compression, vessel wall changes, intraluminal scarification, and enlarged collateral veins. With the patient in an erect position, the femoral, popliteal, and calf veins were examined using a linear array probe (frequency range 3-13 MHz). Inflow of the FV, DFV, and GSV into the CFV was evaluated during flow augmentation. Furthermore, the superficial and deep veins were tested for valvular segmental reflux. Insufficiency was scored as retrograde flow $>1 \mathrm{~s}$ for the deep veins and $>0.5 \mathrm{~s}$ for the superficial veins.

\section{MRV}

A 1.5-Tesla MRI system (Intera, Philips Healthcare, Best, the Netherlands) was used. A dedicated 12 element phased array peripheral vascular coil with cranio-caudal coverage of $128 \mathrm{~cm}$ (Philips) was used for signal reception. Examinations were performed in the supine position. Prior to contrast injection, all patients had a standard two dimensional noncontrast enhanced balanced turbo field echo (BTFE) sequence to visualise the abdominal and pelvic veins. By use of a remote controlled dual head injector (Spectris; Bayer Medrad, Indianola, PA, USA) the contrast agent (Gadobutrol; Gadavist, Bayer HealthCare, Berlin, Germany) was administered 
intravenously at $1.0 \mathrm{ml} / \mathrm{second}(0.2 \mathrm{ml} / \mathrm{kg}$ body weight, equal to $0.2 \mathrm{mmol} / \mathrm{kg})$ in the median cubital vein. Contrast injection was followed by a $20 \mathrm{ml}$ saline flush injection at the same flow rate. Acquisition of the first scan volume was started $30 \mathrm{~s}$ after contrast administration. A three dimensional ultrafast gradient echo sequence (Ultrafast GE, THRIVE, Philips Healthcare) with fat suppression (spectral pre-saturation with inversion recovery) was used for high resolution steady state imaging of the venous system, ensuring coverage of the popliteal veins up to the suprarenal IVC.

\section{Stent placement}

Endovascular interventions were performed in an angiosuite, whereas hybrid procedures were performed in a hybrid operation room. Ultrasound guided venous access was obtained through the ipsilateral mid-femoral vein, below the inflow of the main deep femoral vein branches. In general, a 6-F sheath was introduced to initiate recanalisation. After successfully traversing the guidewire through the obstruction, the 6-F sheath was changed for a 10-F sheath to facilitate balloon angioplasty and stent deployment. Routinely, 5000 IU of heparin were administered before angioplasty. Predilatation was performed in which balloons were sized according to the diameter of the desired stent, generally between 12 and $16 \mathrm{~mm}$, increasing from the CFV to the CIV, and up to $25 \mathrm{~mm}$ for the IVC. The type of stents used varied during the course of the study, on the basis of experience. Without specific indication, stents used included the sinus $\mathrm{XL}$, sinus $\mathrm{XL}$-flex, sinus Venous and sinus Obliquus (Optimed $\mathrm{GmbH}$, Ettlingen, Germany), $\mathrm{VICl}$ (Veniti, St. Louis, MO, USA), Zilver Vena (Cook, Limerick, Ireland), and Venovo (Bard, Covington, GA, USA). Post-dilatation was always performed. Detailed descriptions of the recanalisation and stenting techniques are described elsewhere. ${ }^{14,15,20}$ Post-stenting assessment of the in- and outflow of the treated segments was routinely done by multiplanar venography (Figure 2.1).

\section{Hybrid intervention: endophlebectomy and AVF}

The technical issues and operative techniques of the hybrid procedure have been described in detail elsewhere. ${ }^{21}$ During surgery, the GSV, FV, and branches of the DFV were tested for flow. The presence or absence of spontaneous flow was reported. Afterwards, the CFV was opened, disobliterated, and closed with or without a patch. Since there are no objective measures to quantify adequate flow and the dissected CFV and cranial stents are highly thrombogenic after CFV obliteration, a fistula was created to temporarily increase the flow until re-endothelisation had occurred. ${ }^{16,17,21,22}$ 

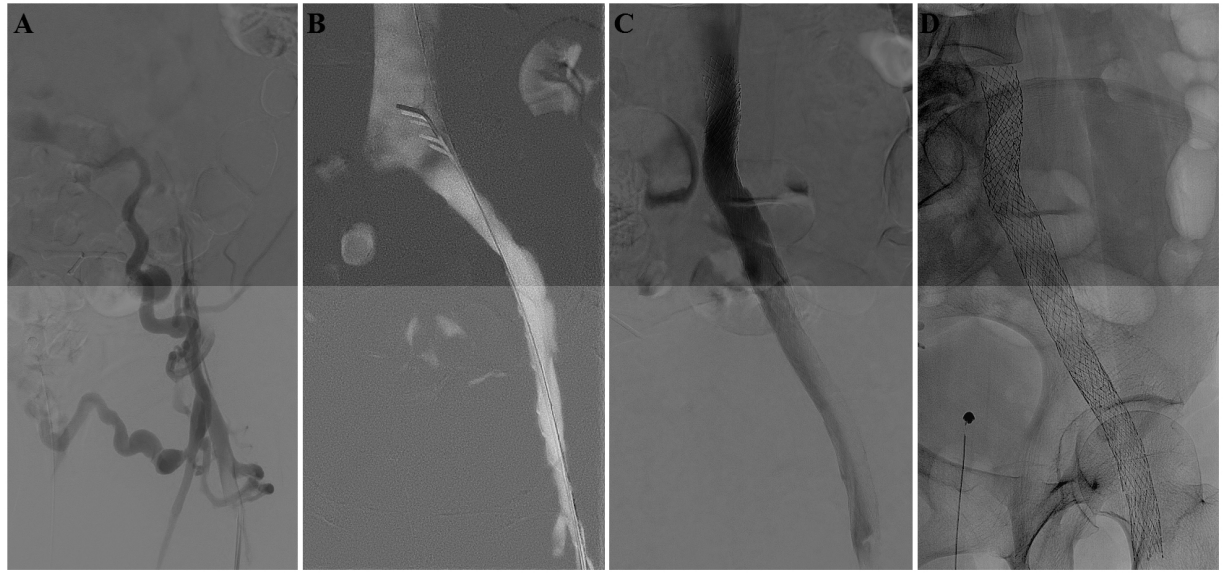

Figure 2.1 (A) Pre-interventional venography of the left iliac tract in a patient with post-thrombotic syndrome who received percutaneous treatment. The proximal iliac tract is not visualised because of lumen occlusion. Multiple collaterals are seen; a secondary sign of vein obstruction. (B) Roadmap image following guidewire recanalisation and percutaneous transluminal angiography of the left iliac tract. Notice the typical translucency in the common iliac vein caused by the compression of the overlying common iliac artery. (C) Venography after stent deployment. Contrast flows freely through the stents. (D) Fluoroscopic image of the stents post-deployment. Stents reach from the proximal common iliac vein into the proximal common femoral vein.

\section{Post-operative care}

All patients received intermittent pneumatic compression during hospitalisation. Patients not using anticoagulants prior to intervention were started directly postintervention on low molecular weight heparins and vitamin $\mathrm{K}$ antagonists, aiming at an international normalised ratio (INR) level of 3-4. Patients using anticoagulants at intervention continued the use during hospitalisation and thereafter aiming at an INR level of 3-4. Anticoagulants were ceased after 6 months if stenosis, stent configuration problems, hypercoagulability, or previous history of recurrent DVT were absent. After the hybrid procedures, a second hospitalisation was planned to close the AVF. Closure of the AVF only took place when there were no stenoses or obstructions of the stented segments or vein segment distal to the stents found with DUS 2-3 weeks postintervention. The aim was to occlude all AVFs within 2 months; however, this was not possible in a number of patients owing to inadequate venous inflow into stents. AVFs were closed using an Amplatzer vascular plug (St Jude Medical, St. Paul, MN, USA) through a contralateral transfemoral arterial access. A minority of AVFs, especially the non-looped or non-polytetrafluoroethylene fistulae, were closed surgically. 


\section{Clinical and DUS surveillance}

After treatment, all clinical scores were re-evaluated. The VCSS was presented as the mean clinical important difference. A decrease in Villalta score of $>2$ points was scored as improvement. A score of -2 or +2 points versus baseline was defined as no improvement. An increase of $>2$ points was scored as worsening. ${ }^{23}$

During follow-up, the same DUS techniques as preintervention were used, with specific attention to stent and, if applicable, AVF patency, presence of lumen diameter reduction of $>50 \%$, presence of post-interventional haematomas, and stent integrity. Features such as stent fracture, kinking, or tapering resulting in lumen reduction and apparent flow impairment were recorded. A suspicion of kinking was scored as any instent angulation causing flow impairment, most frequently caused by rigidity of the stent. When the suspicion of kinking arose, a plain X-ray in three planes was performed.

Tapering was scored as an indentation at the stent end due to external compression, causing flow impairment with filling of collaterals.

\section{Analysis and statistics}

All continuous data are presented as mean $\pm S D$, except for non-normally distributed data (presented as median [range]). Categorical data are presented as frequencies and percentages. An $\alpha$ of $<.05$ was considered statistically significant. Kaplan-Meier survival estimation was used to calculate patency rates. SEM values of $>10 \%$ were discarded as being unreliable and were not reported. Patency analyses were based on the total number of extremities treated, whereas all other calculations were per patient. Clinical scoring data were absent after 24 month follow-up, as these data were available in $<20 \%$ of patients. A clinically relevant difference of minimally 0.5 times the SD of the difference in pre- and posttreatment scores was used as a cutoff value (of 210 [57\%] patients with both a baseline and at least one follow-up score). The study was registered under number NCT02650453 in the clinicaltrials.gov database.

\section{Results}

\section{Demographics}

The mean age of the total population was $43 \pm 14$ years (range 17-77 years) and 257 (70\%) patients were female. Most (261 [93\%]) patients in the P-PTS and H-PTS groups had one or more known DVTs in their medical history. Twenty-one patients (6\%) referred for analysis of chronic venous disease had suffered an occult DVT with postthrombotic vein damage. Patients in the P-PTS and H-PTS groups had a median of 6 (range 1-48) symptomatic years after their first DVT. A minority of IVCS patients was 
also known to have a history of DVT; however, these were distal or contralateral DVTs. The majority (242 [66\%]) of patients were classified as CEAP class COeC3. Treatment indication in these patients was based on the presence of venous claudication and other subjective complaints like pain or oedema. At baseline, venous claudication was present in 33 patients with IVCS (of 83 patients in whom claudication was noted at baseline), 113 P-PTS patients (of 181 patients in whom claudication was noted at baseline) and $70 \mathrm{H}-\mathrm{PTS}$ patients (of 83 patients of whom claudication was noted at baseline).

All patient characteristics are provided per group in Tables 2.1 and 2.2.

Table $2.1 \quad$ Demographics.

\begin{tabular}{lcccc}
\hline & IVCS & P-PTS & H-PTS & Total \\
& $\mathrm{N}=87(24)$ & $\mathrm{N}=196(53)$ & $\mathrm{N}=86(23)$ & $\mathrm{N}=369$ \\
\hline N legs* & $87(21)$ & $221(53)$ & $109(26)$ & 417 \\
Number of females & $74(85)$ & $127(65)$ & $56(65)$ & $257(70)$ \\
Mean age \pm SD (y) & $43 \pm 13$ & $45 \pm 13$ & $40 \pm 15$ & $43 \pm 14$ \\
Single DVT & $18(21)$ & $133(68)$ & $53(62)$ & $204(55)$ \\
Recurrent DVT & $6(7)$ & $44(22)$ & $31(36)$ & $81(22)$ \\
Silent DVT & N.A. & $19(10)$ & $2(2)$ & $21(6)$ \\
History of PE & $5(6)$ & $31(16)$ & $13(15)$ & $49(13)$ \\
Median time between DVT and treatment (y) & N.A. & $7(1-48)$ & $5(1-40)$ & $6(1-48)$ \\
\hline
\end{tabular}

Note. Data are $\mathrm{n}$ (\% of subgroup) unless otherwise indicated. IVCS: iliac vein compression syndrome; P-PTS: post-thrombotic syndrome with percutaneous treatment; H-PTS: post-thrombotic syndrome with hybrid treatment; DVT: deep venous thrombosis; NA: not applicable; PE: pulmonary embolism. * Percentage of total number of legs.

Table 2.2 CEAP classification and reflux.

\begin{tabular}{lcccc}
\hline & IVCS & P-PTS & H-PTS & Total \\
& $\mathrm{N}=87(24)$ & $\mathrm{N}=196(53)$ & $\mathrm{N}=86(23)$ & $\mathrm{N}=369$ \\
\hline N of patients with CEAP & $84(97)$ & $184(94)$ & $83(97)$ & $351(95)$. \\
C-class 0 & $5(6)$ & $16(9)$ & $7(8)$ & $28(8)$ \\
C-class 1 & $10(12)$ & $16(9)$ & $9(11)$ & $35(10)$ \\
C-class 2 & $20(24)$ & $22(12)$ & $10(12)$ & $52(14)$ \\
C-class 3 & $36(43)$ & $60(33)$ & $31(37)$ & $127(34)$ \\
C-class 4a & $6(7)$ & $33(18)$ & $15(18)$ & $54(15)$ \\
C-class 4b & $2(2)$ & $8(4)$ & $4(5)$ & $14(4)$ \\
C-class 5 & $2(2)$ & $12(7)$ & $3(4)$ & $17(5)$ \\
C-class 6 & $4(5)$ & $15(8)$ & $4(5)$ & $23(6)$ \\
Superficial venous reflux & $45(52)$ & $72(37)$ & $12(14)$ & $129(35)$ \\
Deep venous reflux & $12(14)$ & $53(27)$ & $15(17)$ & $80(22)$ \\
Perforator vein reflux & $31(36)$ & $17(9)$ & $12(14)$ & $60(4)$ \\
\hline
\end{tabular}

Note. Data are $\mathrm{n}$ (\% of subgroup) unless otherwise indicated. IVCS: iliac vein compression syndrome; P-PTS: post-thrombotic syndrome with percutaneous treatment; H-PTS: post-thrombotic syndrome with hybrid treatment. 


\section{Intervention}

In 284 (77\%) patients, a left sided intervention was performed, 29 (8\%) had a right sided and $52(14 \%)$ had bilateral intervention. An isolated IVC treatment was performed in four (1\%) patients. Thus, a total of 425 lower extremities were treated. Unilateral disease was treated with a median number of two (range 1-5) stents per patient and a median of five (range 2-8) for bilateral cases. In the H-PTS group 80 (93\%) patients received stenting in combination with endophlebectomy and an AVF. Seven of those 80 patients had a bilateral endophlebectomy and AVF. Four patients were stented with an endophlebectomy without an AVF and two patients received stenting with an AVF but without an endophlebectomy. A bovine patch was used when closing the venotomy in 42 (49\%) patients.

\section{Patency rates and re-interventions}

Primary patency rates at the 60 month follow-up were $90 \%$ and $64 \%$ for the IVCS and P-PTS groups, respectively. Assisted primary patency rates were $100 \%$ and $81 \%$, and secondary patency rates $100 \%$ and $89 \%$, respectively. The HPTS group had a shorter follow-up of 36 months showing a primary, assisted primary, and secondary patency rate of $37 \%, 62 \%$, and $72 \%$, respectively. Patency rates are shown in Figure 2.2(A-D).

In the IVCS group there were no thrombotic stent occlusions. Primary patency loss was due to re-interventions of persistent stenoses. In the P-PTS population 36 (18\%) patients had a thrombotic stent occlusion. These thrombotic stent occlusions were treated by thrombolysis with secondary stenting in 19 patients, of whom two received thrombolysis twice and two required a thrombectomy. The remaining 15 thrombotic stent occlusions did not induce severe complaints interfering with daily activities and therefore did not receive an additional intervention. In the H-PTS group 33 (38\%) patients suffered a thrombotic stent occlusion, of whom 10 (12\%) received thrombolysis. Sixteen cases (8\%) in the P-PTS group and 10 cases $(12 \%)$ in the HPTS group required a secondary open surgical procedure (revision or additional endophlebectomy and/or AVF) to optimise inflow into the stents. Re-stenting for new or previously unrecognised lesions, residual compression, or stent related issues was performed in seven (8\%), 49 (25\%), and 46 (53\%) cases in the IVCS, P-PTS, and H-PTS groups, respectively (Table 2.3 ).

All in all, the majority of patients who were treated in a hybrid fashion needed at least one re-intervention (59\%) versus $27 \%$ in the P-PTS and $8 \%$ in the IVCS groups. In addition, they needed the first re-interventions earlier after their primary intervention compared with the IVCS group. The median number of days between the first and second intervention in the IVCS group was 143 days (range 20-300 days) versus 140 days (range 17-355 days) in the P-PTS group and 48 days (range 11-115 days) for the H-PTS group. 
A Patency rates total population

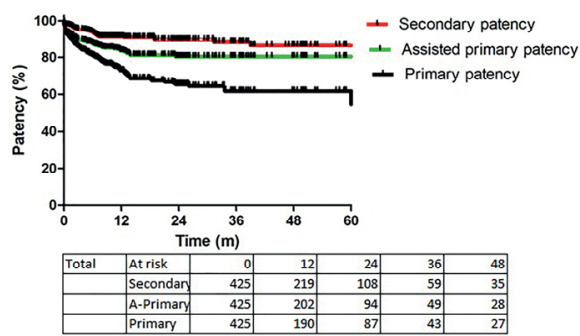

B

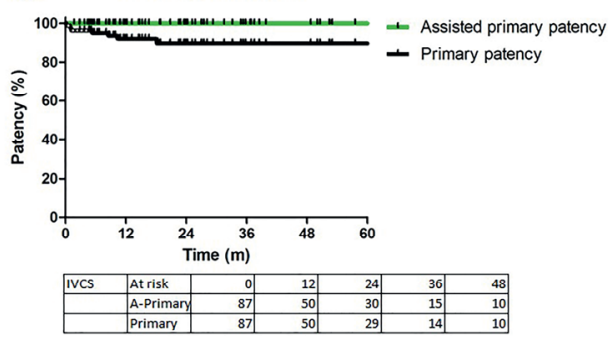

C Patency rates P-PTS

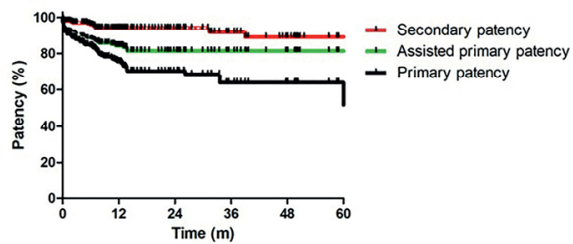

\begin{tabular}{|l|l|r|r|r|r|r|}
\hline P-PTS & At risk & 0 & 12 & 24 & 36 & 48 \\
\hline & Secondan & 229 & 114 & 61 & 37 & 22 \\
\hline & A-Primary & 229 & 106 & 52 & 30 & 18 \\
\hline & Primary & 229 & 101 & 49 & 27 & 17 \\
\hline
\end{tabular}

D

Patency rates H-PTS

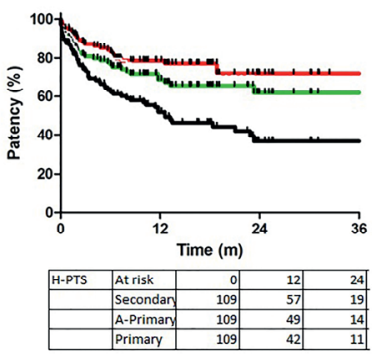

I Secondary patency - Assisted primary patency - Primary patency

Figure 2.2 Cumulative patency rates of stented limbs in the total population, and of the iliac vein compression syndrome (IVCS), percutaneous post-thrombotic syndrome (P-PTS) and postthrombotic syndrome with hybrid treatment (H-PTS; definitions explained in text) subgroups. The number at risk at each interval is given below the graph. All SEM are $<10 \%$. (A) Patency rates of the total population. (B) Patency rates of the IVCS subpopulation. (C) Patency rates of the P-PTS subpopulation. (D) Patency rates of the H-PTS subpopulation.

\section{Other complications}

The most frequent complications were thrombotic stent occlusion and restenosis (Table 2.4). Contralateral DVT was rare and only seen in two patients (1\%). Mortality was zero and there were no instances of clinical pulmonary embolism. However, minor and major bleeding, necessitating supplementary treatment or re-intervention, was seen after treatment of $32(9 \%)$ and $13(4 \%)$ patients, respectively, and occurred mainly in the H-PTS group. Of special concern in the hybrid subpopulation was the high incidence of wound related complications, such as wound infections in 23 (27\%), lymphorrhoea in $28(33 \%)$, and wound dehiscence in 10 (12\%) patients. 
Table 2.3 Number of re-interventions per patient.

\begin{tabular}{lcccc}
\hline & IVCS & P-PTS & H-PTS & Total \\
& $\mathbf{N = 8 7 ( 2 4 )}$ & $\mathbf{N = 1 9 6 ( 5 3 )}$ & $\mathbf{N = 8 6 ( 2 3 )}$ & $\mathbf{N = 3 6 9}$ \\
\hline None & $80(92)$ & $144(74)$ & $35(41)$ & $259(70)$ \\
$>1$ reintervention & $0(0)$ & $22(11)$ & $19(22)$ & $41(11)$ \\
Only PTA & $0(0)$ & $11(6)$ & $10(12)$ & $21(6)$ \\
CD-Thrombolysis & $0(0)$ & $21(11)$ & $10(12)$ & $31(8)$ \\
Thrombectomy & $0(0)$ & $2(1)$ & $3(4)$ & $5(1)$ \\
Restenting & $7(8)$ & $38(19)$ & $42(49)$ & $87(24)$ \\
Restenting + thrombectomy & $0(0)$ & $3(2)$ & $2(2)$ & $5(1)$ \\
Restenting + AVF & $0(0)$ & $1(1)$ & $1(1)$ & $2(1)$ \\
Restenting + endophlebectomy + AVF & $0(0)$ & $7(4)$ & $0(0)$ & $7(2)$ \\
AVF + endophlebectomy + restenting & $0(0)$ & $0(0)$ & $1(1)$ & $1(<1)$ \\
After hybrid procedure & & & & \\
Endophlebectomy & $0(0)$ & $0(0)$ & $1(1)$ & $1(<1)$ \\
Creating AVF & $0(0)$ & $4(2)$ & $1(1)$ & $5(1)$ \\
AVF + thrombectomy & $0(0)$ & $2(1)$ & $0(0)$ & $2(1)$ \\
AVF + endoflebectomy + bypass & $0(0)$ & $2(1)$ & $0(0)$ & $2(1)$ \\
Revision AVF + Thrombectomy & N.A. & N.A. & $6(7)$ & $6(2)$ \\
Excision PTFE AVF & N.A. & $2(1)$ & $4(4)$ & $6(2)$ \\
Transposition & $0(0)$ & $2(1)$ & $2(2)$ & $4(1)$ \\
Bypass & $0(0)$ & $0(0)$ & $1(1)$ & $1(<1)$ \\
\hline
\end{tabular}

Note. Data are $\mathrm{n}$ (\% of subgroup) unless otherwise indicated. IVCS: iliac vein compression syndrome; P-PTS: post-thrombotic syndrome with percutaneous treatment; H-PTS: post-thrombotic syndrome with hybrid treatment; PTA: percutaneous transluminal angioplasty; CD: catheter directed; AVF: arteriovenous fistula; N.A.: not applicable; PTFE: polytetrafluoroethylene.

\section{Clinical scores}

Significant improvement in VCSS was seen in all groups during the first 24 months of follow-up (Figure 2.3). A significant improvement of Villalta scale was seen for the postthrombotic patients. A clinically positive treatment effect was seen in $63 \%, 72 \%$, and $73 \%$ of patients in the IVCS, PPTS, and H-PTS groups, respectively. No response to therapy was seen in $24 \%, 23 \%$, and $22 \%$ of patients, respectively. Worsening of symptomatology was observed in $14 \%, 5 \%$, and $6 \%$ of IVCS, P-PTS, and H-PTS patients. A separate analysis for this non-response group of patients will be performed in future research.

Venous claudication was calculated in those patients where a baseline score and at least one follow-up score was available. This reduced in 26/29 (90\%) patients with IVCS after a median follow-up of 17 months (range 12-26 months). In 64/78 (82\%) P-PTS patients venous claudication was absent after a median of 13 months (range 11-24 months) and in 50/60 (83\%) H-PTS patients after 16 months (range 11-25 months). During follow-up, ulceration healed in 20/23 (87\%) of the patients presenting with an active ulcer at baseline. However, ulcer recurrence was seen in four (20\%) patients. 
Table 2.4 Complications per patient.

\begin{tabular}{|c|c|c|c|c|}
\hline & $\begin{array}{c}\text { IVCS } \\
\mathrm{N}=87(24)\end{array}$ & $\begin{array}{c}\text { P-PTS } \\
\mathrm{N}=196(53)\end{array}$ & $\begin{array}{c}\mathrm{H}-\mathrm{PTS} \\
\mathrm{N}=86(23)\end{array}$ & $\begin{array}{c}\text { Total } \\
\mathrm{N}=369\end{array}$ \\
\hline None & $63(73)$ & $98(50)$ & $25(29)$ & $186(50)$ \\
\hline Thrombotic stent occlusion & $0(0)$ & $36(18)$ & $33(38)$ & 69 (19) \\
\hline Stent stenosis & $2(2)$ & $30(15)$ & $24(28)$ & $56(15)$ \\
\hline Residual stent compression & $16(18)$ & $14(7)$ & $4(5)$ & $34(9)$ \\
\hline Minor bleeding & $2(2)$ & $16(8)$ & $14(16)$ & $32(9)$ \\
\hline Major bleeding & $0(0)$ & $2(1)$ & $11(13)$ & $13(4)$ \\
\hline Aneurysma spurium & $1(1)$ & $0(0)$ & $1(1)$ & $2(1)$ \\
\hline DVT distally of stents & $0(0)$ & $1(1)$ & $3(4)$ & $4(1)$ \\
\hline Contralateral DVT & $0(0)$ & $1(1)$ & $1(1)$ & $2(1)$ \\
\hline Thrombophlebitis & $1(1)$ & $2(1)$ & $0(0)$ & $3(1)$ \\
\hline Anaphylactoid reaction to contrast & $0(0)$ & $0(0)$ & $0(0)$ & $1(<1)$ \\
\hline \multicolumn{5}{|l|}{ Non-specific } \\
\hline Urinary tract infection & $0(0)$ & $2(1)$ & $0(0)$ & $2(1)$ \\
\hline Priapism LMWH & $0(0)$ & $1(1)$ & $0(0)$ & $1(<1)$ \\
\hline Urinary retention & $0(0)$ & $1(1)$ & $0(0)$ & $1(<1)$ \\
\hline \multicolumn{5}{|l|}{ Stent integrity issues } \\
\hline Tapering & $1(1)$ & $11(6)$ & $5(6)$ & $17(5)$ \\
\hline Kinking & $0(0)$ & $3(2)$ & $1(1)$ & $4(1)$ \\
\hline Fracture & $0(0)$ & $1(1)$ & $0(0)$ & $1(<1)$ \\
\hline Angulation & $0(0)$ & $6(3)$ & $3(4)$ & $9(2)$ \\
\hline Implosion & $0(0)$ & $4(2)$ & $1(1)$ & $5(1)$ \\
\hline \multicolumn{5}{|l|}{ Open-surgery related } \\
\hline Wound infection & N.A. & $5 *(3)$ & $23(27)$ & $28(8)$ \\
\hline Seroma & N.A. & $1 *(1)$ & $5(6)$ & $6(2)$ \\
\hline lymphorrhea & N.A. & $1^{*}(1)$ & $28(33)$ & $29(8)$ \\
\hline Wound dehiscention & N.A. & $0(0)$ & $10(12)$ & $10(3)$ \\
\hline
\end{tabular}

Note. Data are $\mathrm{n}$ (\% of subgroup) unless otherwise indicated. IVCS: iliac vein compression syndrome; P-PTS: post-thrombotic syndrome with percutaneous treatment; H-PTS: post-thrombotic syndrome with hybrid treatment; DVT: deep venous thrombosis; LMWH: low molecular weight heparin; NA: not applicable. ${ }^{*}$ Related to secondary open surgical procedures. N.A. not apllicable 

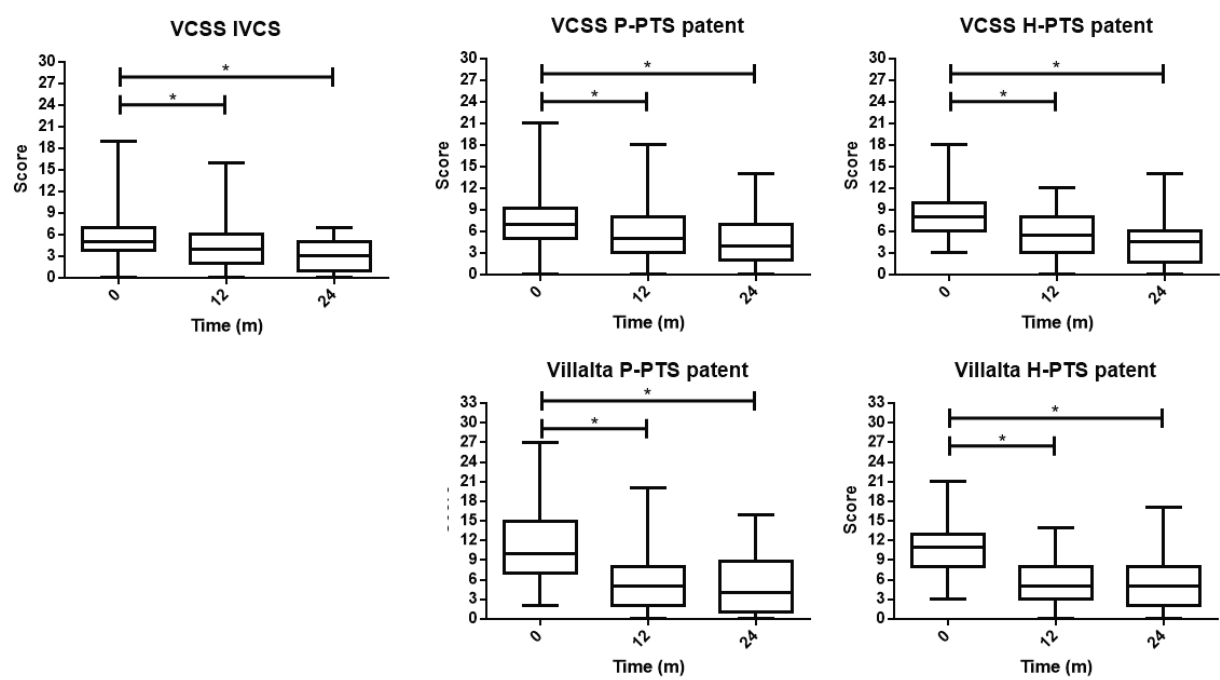

Figure 2.3 Venous Clinical Severity Score (VCCS) and Villalta score of the three subpopulations given as clinical scores over 24 months. Note. IVCS: iliac vein compression syndrome; P-PTS: percutaneous post-thrombotic syndrome; H-PTS: post-thrombotic syndrome with hybrid treatment.

\section{Discussion}

In this study, the routine clinical use of stenting in 369 patients with PTS and IVCS is described.

Patency rates in patients with IVCS were 90\%, 100\%, and $100 \%$ at 60 months and were comparable with previous studies. ${ }^{2,3,4}$ The main patency related issues were allied to stent geometry and stent placement problems. Regarding this, future outcomes are expected to improve even more with the introduction of dedicated venous stents, as excellent results of a dedicated venous stent during 12 months follow-up already support this. ${ }^{14}$

In contrast to the IVCS group, the post-thrombotic subgroups did show stent occlusions. However, secondary patency rates in the P-PTS group were shown to be $89 \%$ and comparable with results described in previous reviews. ${ }^{2,3,4}$ The primary patency of $64 \%$ in P-PTS patients was most likely lower as a result of inadequate inflow. This is exemplified by the fact that venography during re-interventions frequently showed residual post-thrombotic stenosis distal to the stents. Moreover, patency rates in H-PTS group of $37 \%, 62 \%$, and $72 \%$ at 36 months, proved that impaired flow due to prior thrombosis of the vein tracts below the CFV reduces patency outcomes. 
Currently, there is no definitive test available to evaluate the adequacy of inflow prior to stenting or directly after deployment of stents. For this reason, clinical judgment must be relied upon. In general, it is believed that post-thrombotic changes of the CFV below the SFJ with affected orifices of FV and DFV provide inadequate inflow and need a hybrid procedure. Future research should focus on a scoring system to quantify the flow. Afterwards, the evaluation of a cutoff flow value to predict stent inflow and related patency might be performed. Next, decision making based on such a haemodynamic evaluation of DUS, MRV, venography, and IVUS images should be evaluated with respect to the stent patency and clinical outcome. Moreover, if treatment type can be predicted more precisely it is possible to overcome complication related issues, as seen in the H-PTS group.

Since patients in the H-PTS group were more prone to several surgery related complications, like wound infections $(27 \%)$ and wound dehiscence $(12 \%)$, the primary patency rate was negatively influenced. Because of the recently disobliterated CFV, this area is more prone to compression by haematomas or lymphocoeles (33\%). Moreover, deployed stents are inherently thrombogenic specifically in combination with the stent material and the exposed collagen of the disobliterated area. Furthermore, the AVF may potentially induce intimal hyperplasia causing stenosis of the distal stents or stenosis of the disobliterated area.

In all, but especially in patients treated for PTS, the quality of anticoagulation might be an issue, as it is known that INR values can be inconsistent and influence stent patency. Therefore, a post-operative INR target range of 3-4 (in accordance with Dutch guidelines) was chosen. Since INR levels are regulated by different specialised entities, an analysis of all INR levels was not possible. Of further note is the fact that interventions, including the surgical disobliteration, were performed on patients already anticoagulated.

This has the disadvantage of increasing the rate of post-operative bleeding, though it is believed it is necessary to reduce the risk of early thrombotic stent occlusion.

To prevent such an acute stent occlusion, all patients are treated with a pre-operative INR level of 2.0-2.5. Oral anticoagulants were stopped 24 hours before the intervention to prevent massive haematomas.

Patients with severe post-thrombotic obstructions, needing a hybrid intervention, have a high risk of complications and re-interventions. However, the hybrid treatment may improve quality of life and pain, and significantly reduce venous claudication when successful. It may be offered to a select group of patients with marked impairment of quality of life and debilitating complaints interfering with daily activities.

The hybrid procedure should be discussed with the patient in detail, explaining all possible complications, to make an adequate balanced risk/benefit analysis.

Although favourable results have been presented, there are some limitations to this research, which might cause some bias. As there are no large clinical trials to compare 
the H-PTS outcomes with, clinical outcomes were partly comparable with previous research, but no response to therapy was seen in $24 \%$ of IVCS and $23 \%$ of P-PTS patients, respectively. ${ }^{2,3,4}$ Besides this, worsening of complaints was shown in $14 \%$ and $5 \%$ of patients, respectively. As venous reflux was present at baseline in many patients and was not routinely assessed during follow-up, this non-response might be explained by residual reflux.

In addition, the incompleteness of clinical score data may be a bias in analyzing the treatment effect. Furthermore, especially in the cases of non-response to stenting in IVCS, an initial indication issue might be present. As symptomatic venous disease and anatomical compression of the iliac veins are both common in the Western population, in some patients the compression might not have been the reason for the symptoms. ${ }^{11}$ Stenting in these patients can be seen as a type of overtreatment. As currently no validated haemodynamic tests exist to assess the relative influence of the iliac vein compression on the lower extremity, it is impossible to differentiate between symptomatic chronic venous compression and anatomical variance in some patients. Future prospective trials should take the abovementioned issues into account to improve optimal care in patients with deep venous obstruction.

\section{Conclusion}

Percutaneous stent placement to treat non-thrombotic iliac vein lesions, and postthrombotic ilio-femoral obstructions is safe, effective, and showed patency rates comparable with previous research. Patients with advanced disease needing a hybrid procedure showed lower patency rates and more complications. However, when successful, the clinical outcome is favourable at mid-term follow-up and the procedure may be offered to selected patients. 


\section{References}

1. Neglen P, Hollis KC, Olivier J, Raju S. Stenting of the venous outflow in chronic venous disease: longterm stent-related outcome, clinical, and hemodynamic result. J Vasc Surg. 2007;46(5):979-90.

2. Seager MJ, Busuttil A, Dharmarajah B, Davies AH. Editor's Choice-- A Systematic Review of Endovenous Stenting in Chronic Venous Disease Secondary to Iliac Vein Obstruction. Eur J Vasc Endovasc Surg. 2016;51(1):100-20.

3. Wen-da W, Yu Z, Yue-Xin C. Stenting for chronic obstructive venous disease: A current comprehensive meta-analysis and systematic review. Phlebology. 2015.

4. Razavi MK, Jaff MR, Miller LE. Safety and Effectiveness of Stent Placement for Iliofemoral Venous Outflow Obstruction: Systematic Review and Meta-Analysis. Circ Cardiovasc Interv. 2015;8(10): e002772.

5. Wittens C, Davies AH, Baekgaard N, Broholm R, Cavezzi A, Chastanet S, et al. Editor's Choice Management of Chronic Venous Disease: Clinical Practice Guidelines of the European Society for Vascular Surgery (ESVS). Eur J Vasc Endovasc Surg. 2015;49(6):678-737.

6. Kahn SR, Shbaklo H, Lamping DL, Holcroft CA, Shrier I, Miron MJ, et al. Determinants of health-related quality of life during the 2 years following deep vein thrombosis. J Thromb Haemost. 2008;6(7): 1105-12.

7. Enden T, Haig Y, Klow NE, Slagsvold CE, Sandvik L, Ghanima W, et al. Long-term outcome after additional catheter-directed thrombolysis versus standard treatment for acute iliofemoral deep vein thrombosis (the CaVenT study): a randomised controlled trial. Lancet. 2012;379(9810):31-8.

8. Meissner MH, Eklof B, Smith PC, Dalsing MC, DePalma RG, Gloviczki P, et al. Secondary chronic venous disorders. J Vasc Surg. 2007;46 Suppl S:68s-83s.

9. May R, Thurner J. The cause of the predominantly sinistral occurrence of thrombosis of the pelvic veins. Angiology. 1957;8(5):419-27.

10. Cockett FB, Thomas ML. The iliac compression syndrome. Br J Surg. 1965;52(10):816-21.

11. Kibbe MR, Ujiki M, Goodwin AL, Eskandari M, Yao J, Matsumura J. Iliac vein compression in an asymptomatic patient population. J Vasc Surg. 2004;39(5):937-43.

12. Kahn SR, Shrier I, Julian JA, Ducruet T, Arsenault L, Miron MJ, et al. Determinants and time course of the postthrombotic syndrome after acute deep venous thrombosis. Annals of internal medicine. 2008;149(10):698-707.

13. Neglen P, Tackett TP, Jr., Raju S. Venous stenting across the inguinal ligament. J Vasc Surg. 2008;48(5):1255-61.

14. de Wolf MA, de Graaf R, Kurstjens RL, Penninx S, Jalaie H, Wittens $\mathrm{CH}$. Short-Term Clinical Experience with a Dedicated Venous Nitinol Stent: Initial Results with the Sinus-Venous Stent. Eur J Vasc Endovasc Surg. 2015.

15. Friedrich de Wolf MA, Arnoldussen CW, Grommes J, Hsien SG, Nelemans PJ, de Haan MW, et al. Minimally invasive treatment of chronic iliofemoral venous occlusive disease. Journal of Vascular Surgery: Venous and Lymphatic Disorders. 2013;1(2):146-53.

16. Vogel D, Comerota AJ, Al-Jabouri M, Assi Zl. Common femoral endovenectomy with iliocaval endoluminal recanalization improves symptoms and quality of life in patients with postthrombotic iliofemoral obstruction. J Vasc Surg. 2012;55(1):129-35.

17. Puggioni A, Kistner RL, Eklof B, Lurie F. Surgical disobliteration of postthrombotic deep veins-endophlebectomy--is feasible. Journal of vascular surgery : official publication, the Society for Vascular Surgery [and] International Society for Cardiovascular Surgery, North American Chapter. 2004;39(5):1048-52; discussion 52.

18. Comerota AJ, Grewal NK, Thakur S, Assi Z. Endovenectomy of the common femoral vein and intraoperative iliac vein recanalization for chronic iliofemoral venous occlusion. J Vasc Surg. 2010;52(1):243-7.

19. Eklof B, Rutherford RB, Bergan JJ, Carpentier PH, Gloviczki P, Kistner RL, et al. Revision of the CEAP classification for chronic venous disorders: consensus statement. J Vasc Surg. 2004;40(6):1248-52. 
20. de Graaf R, de Wolf M, Sailer AM, van Laanen J, Wittens C, Jalaie H. Iliocaval Confluence Stenting for Chronic Venous Obstructions. Cardiovasc Intervent Radiol. 2015.

21. de Wolf MA, Jalaie H, van Laanen JH, Kurstjens RL, Mensinck MJ, de Geus MJ, et al. Endophlebectomy of the common femoral vein and arteriovenous fistula creation as adjuncts to venous stenting for post-thrombotic syndrome. Br J Surg. 2017;104(6):718-25.

22. Raju S, Neglen P, Doolittle J, Meydrech EF. Axillary vein transfer in trabeculated postthrombotic veins. Journal of vascular surgery : official publication, the Society for Vascular Surgery [and] International Society for Cardiovascular Surgery, North American Chapter. 1999;29(6):1050-62; discussion 62-4.

23. Norman GR, Sloan JA, Wyrwich KW. The truly remarkable universality of half a standard deviation: confirmation through another look. Expert Rev Pharmacoecon Outcomes Res. 2004;4(5):581-5. 


\section{Chapter 3}

Patency rates and clinical outcomes in a cohort of 200 patients treated with a dedicated venous stent 


\section{Abstract}

\section{Objective}

Minimally invasive interventions by recanalization, percutaneous transluminal angioplasty, and stenting in post-thrombotic syndrome (PTS) obstructions and iliac vein compression syndrome (IVCS) have shown good results. Until recently, no dedicated venous stents were available, and stent-related issues accounted for a decrease in patency scores. The introduction of dedicated stents with more flexibility and higher radial forces could result in higher patency scores. This study focused on the outcomes of patients treated by a dedicated sinus-Venous stent (OptiMed $\mathrm{GmbH}$, Ettlingen, Germany). Patency rates and clinical evaluation are described for both PTS and IVCS patients.

\section{Methods}

A total of 200 patients treated at a tertiary university referral center were analyzed. A percutaneous procedure was performed in 103 (51\%) PTS patients and 48 (24\%) IVCS patients. In $49(25 \%)$ patients, a hybrid procedure was executed. Patency rates and complications were analyzed by duplex ultrasound. Clinical improvement was scored by Venous Clinical Severity Score, Villalta scale, and venous claudication rates.

\section{Results}

The mean age was $43.2 \pm 14.5(17-81)$ years, and $66 \%$ were female. Mean Villalta score decreased from $10.5 \pm 4.2(3-24)$ to $5.3 \pm 3.8(0-14)$ at the latest follow-up $(P<.001)$. Venous Clinical Severity Score decreased by a total of 3 points $(P<.001)$. At baseline, venous claudication was present in 132 patients, which subsided in 115 (87\%). Overall patency scores revealed a primary patency of $68 \%$, assisted primary patency of $83 \%$, and secondary patency of $90 \%$ with a median follow-up of 12 (11-12) months. Of all included patients, $122(61 \%)$ did not have a complication during follow-up; the most frequent complications were in-stent restenosis $(n=23)$ and occlusion $(n=25)$ of the stented tract.

\section{Conclusions}

Short-term clinical results using the sinus-Venous stent are comparable to previous research. Loss of stent patency due to stent-related issues like kinking or tapering is hardly ever seen in this short-term follow-up. 


\section{Introduction}

Chronic venous cavoiliofemoral obstruction can cause debilitating symptoms interfering with daily activities and decreasing patients' quality of life. ${ }^{1}$ In the recent past, the most promising treatment consisted of pain medication, compression stockings, and anticoagulation; bypass surgery was reserved only for severely affected cases. ${ }^{2}$ With the introduction of minimally invasive interventions by recanalization, percutaneous transluminal angioplasty (PTA), and stenting, treatment has been revolutionized. In both post-thrombotic syndrome (PTS) obstructions with intraluminal scarring tissue after a deep venous thrombosis (DVT) and iliac vein compression syndrome (IVCS), good results have been reported. ${ }^{3-5}$

Previous systematic reviews have shown high technical success up to $98 \%$ and a low complication rate (0\%-9\%). ${ }^{3-5}$ Restoration of the natural venous outflow tract, by PTA and stenting, reduces venous hypertension and consequently leads to a reduction of clinical symptoms. ${ }^{6}$ In previous literature, reduced venous pressure has been shown after interventional treatment. ${ }^{7}$ Most notably, this is the main factor in the relief of edema by $64 \%$ to $68 \%$, pain relief in up to $69 \%$ to $82 \%$, and ulcer healing rate of $56 \%$ to $100 \%$. With this relatively new indication of endovenous stenting, a primary patency rate between $32 \%$ and $99 \%$ and a secondary patency rate of $66 \%$ to $96 \%$ have been reported. Even so, clinical severity scores showed statistically significant decreases of $>50 \%(P<.0001)$ in multiple cohort studies. ${ }^{3-5}$

Initially, treatment was performed with stents developed for the arterial system. However, these stents show specific characteristics to overcome the negative effects of atherothrombotic plaques. In the venous system, the main pathophysiologic issue consists of intraluminal vein scarification and is not related to atherothrombotic plaques. $^{8}$ Subsequently, dedicated venous stents were developed with favorable features for the venous system.

The main goal of these stents is to counter extraluminal compression with or without intraluminal vessel wall scarification. With the procured benefits of a one-piece nitinol, high-grade electropolished stent, high radial forces and more flexibility can be provided. Moreover, these stents are equipped with radiopaque markers with a smooth running pullback technique that will make deployment of stents easier. Therefore, it is expected that stent-related complications like in-stent restenosis, kinking, and tapering will occur less frequently. ${ }^{9}$ Furthermore, appropriate stent sizes make it possible to treat long venous segments. Currently, different types of dedicated venous stents have been developed. Large prospective trials will show whether these stent types have fewer complications and acceptable patency rates. In small cohort studies, favorable results have already been shown. ${ }^{10-12}$

This study focused on the outcomes of patients treated by a dedicated sinus-Venous stent (OptiMed GmbH, Ettlingen, Germany). Patency rates and clinical evaluation are described for both PTS patients and patients with IVCS. 


\section{Methods}

All patients treated with a sinus-Venous stent (Figure 3.1) at a tertiary university hospital between 2012 and July 2015 were included. A total of 200 patients with unilateral or bilateral complaints of venous outflow obstruction due to postthrombotic changes or iliac vein compression, irrespective of Clinical, Etiology, Anatomy, and Pathophysiology (CEAP) classification, were taken into account. Patients were eligible for treatment when related complaints consisted of pain, heaviness, cramps, edema, or venous claudication interfering with daily activities. Venous claudication was defined as the onset or worsening of pain during ambulation that subsided during rest, especially on resting or elevating the legs, and scored as either being present or not. In $31 \%$ of all patients, a CEAP clinical class between CO and C2 was found, in which the driving force for treatment was debilitating complaints scored as venous claudication.

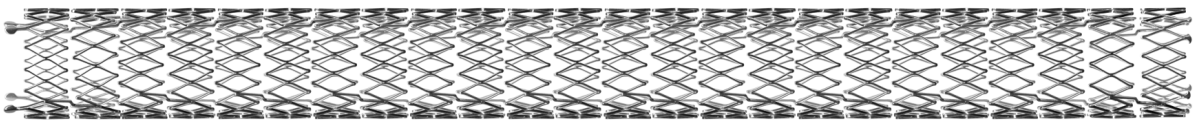

Figure 3.1 The Sinus Venous stent (Optimed GmbH, Ettlingen, Germany). Radiopaque markers shown at cranial part of the stent.

Beside clinical complaints, signs of deep venous obstruction on additional imaging were indispensable to be eligible for treatment. To analyze the extent of postthrombotic changes at baseline, all patients had a duplex ultrasound (DUS) and magnetic resonance venography (MRV) screening. A thorough description of DUS and MRV imaging techniques has been published previously. ${ }^{13}$ For the IVCS patients, these results were combined with phlebography. IVCS was referred to as an external compression of $>50 \%$ lumen reduction in the presence of a collateral network, better detected with venography than with intravascular ultrasound (IVUS), and related clinical complaints. ${ }^{14}$ In this group, none of the patients experienced a previous DVT in the index leg, and no post-thrombotic scarification or vein wall fibrosis was apparent. All PTS patients had one or multiple DVTs in the index leg with additional vein wall fibrosis or intraluminal scarification. The treatment of this group of patients was subdivided into a sole percutaneous treatment or a more extensive hybrid treatment with an endophlebectomy and arteriovenous fistula (AVF). The decision between an anticipated sole percutaneous treatment and more extensive hybrid treatment was made according to the DUS and MRV results. When PTS obstruction was limited to segments central to the saphenofemoral junction in the common femoral vein (CFV) or when a trabeculation-free landing zone in the CFV between the saphenofemoral 
junction and the deep femoral vein (DFV) and femoral vein (FV) confluence was identified, a percutaneous procedure was thought to be sufficient. Otherwise, a hybrid procedure was planned. Moreover, when the orifices of the FV and the DFV were occluded, patients were thought to be in need of the hybrid procedure. Roadmap phlebography was performed at the start of the intervention, and definitive differentiation was made intraoperatively.

An exclusion criterium for this study was a history of DVT $<12$ months ago as sufficient natural recanalization might still occur. ${ }^{15}$

\section{Scoring and follow-up}

Follow-up of stent patency was standardized for all patients at 2 and 6 weeks and 3, 6, and 12 months after intervention. Clinical scores were evaluated by the treating physician and filled out at the expense of the scoring doctor. At baseline and during follow-up, the highest clinical class of CEAP, Venous Clinical Severity Score (VCSS), and venous claudication were scored for IVCS and PTS patients. For all PTS patients, the Villalta score was noted at baseline and during follow-up. As this is a retrospective analysis and the scores were evaluated by the treating physician, there are some missing values. In all treated patients, at least one of the clinical scores was available. The number of available and entered data are recorded for each value.

The patency of stents was analyzed by DUS, in which the existence of any antegrade flow in the stents and spontaneous Doppler signal were scored as a patent stent tract. Specifically, attention was given to the presence of persistent external stent compression, in-stent restenosis of any degree, postinterventional hematomas, and stent integrity giving flow impairment. Kinking was scored as any in-stent angulation causing flow impairment, which was caused by rigidity of the stent in the majority of cases. When this was noted, an abdominal radiograph in anteroposterior, lateral, left and right, and oblique directions was performed to prove it. Tapering was scored as an indentation at the stent end due to external compression, causing a flow impairment.

\section{Interventions}

All interventional procedures have been described previously. ${ }^{16,17}$ In short, patients with IVCS were treated by PTA and stenting using local anesthesia. PTS patients with scarification of caval, common iliac vein (CIV), or external iliac vein cranial to the confluence of the FV and DFV were treated under sedation (percutaneous PTS group). In patients with post-thrombotic changes extending to the CFV or in whom it was suspected that stenting of the CFV would displace intraluminal tissue across the orifices of the FV and DFV, an endophlebectomy of the CFV was chosen. Afterward, a 
6-mm polytetrafluoroethylene graft, looplike AVF was created between the CFV and common femoral artery (hybrid PTS group). ${ }^{17}$

Predilation was performed in which balloons were sized according to the diameter of the desired stent, generally somewhat oversized between 12 and $18 \mathrm{~mm}$ increasing from the CFV to the CIV and up to $24 \mathrm{~mm}$ for the inferior vena cava (Maxi LD; Cordis, Fremont, Calif). If the vein did not expand with angioplasty, a highpressure balloon was used to achieve adequate predilation. The sinus-Venous (OptiMed) stents were used in the CIV, external iliac vein, CFV, and DFV. Iliac stents were positioned at least at the level of the CIV confluence, generally extending to the spinous process (Figure 3.2).
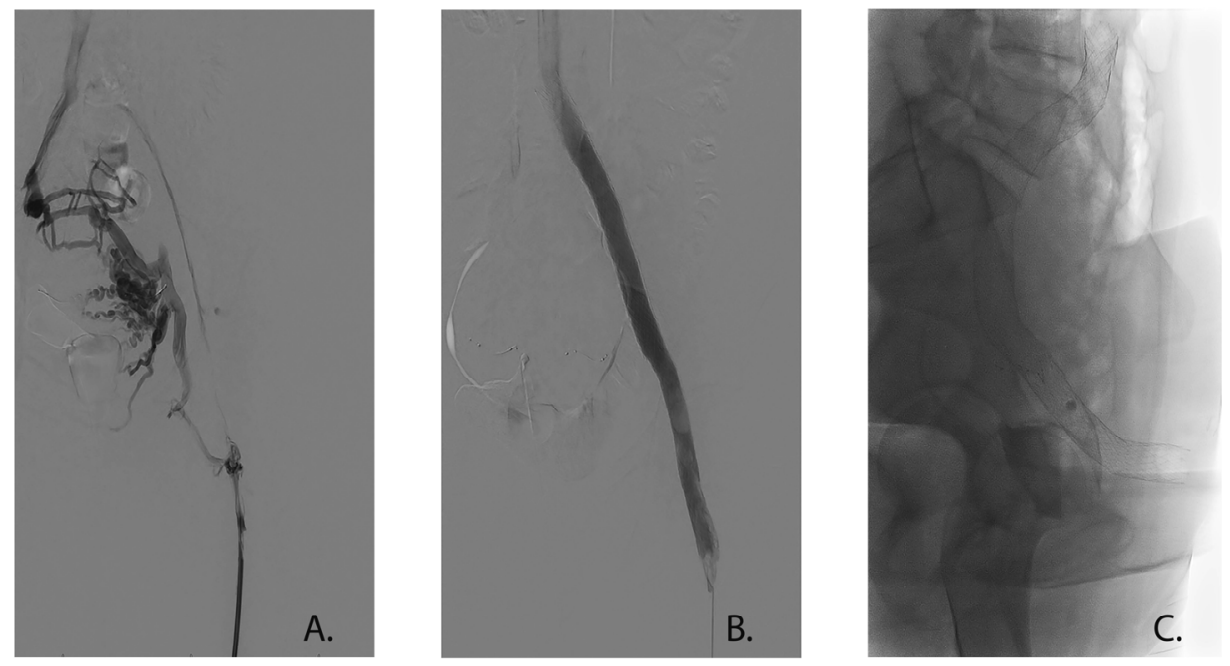

Figure 3.2 Example of intervention. (A) Roadmap venography showing iliofemoral occlusion on the left side and collateral flow. (B) Image of completion venography showing adequate inflow through the extended stent into iliac stents. Collateral pathways disappeared. (C) Lateral view of deployed stents showing the high flexibility.

The iliac part was stented with stents of $18 \times 60$ to $80 \mathrm{~mm}, 16 \times 40$ to $150 \mathrm{~mm}$, or $14 \times 40$ to $150 \mathrm{~mm}$, depending on the vein lumen after ballooning. The FV was stented with $14 \times 40$ to $150 \mathrm{~mm}, 12 \times 40$ to $150 \mathrm{~mm}$, or $10 \times 40$ to $100 \mathrm{~mm}$ stents. For the caval obstructions, a sinus-XL 24x80 to $160 \mathrm{~mm}$ (OptiMed) was used as these stents were available in larger diameters. Next, postdilation was performed with the same balloons used for predilation.

During interventions, heparin (5000 units) was administered once to all patients. Afterward, the anticoagulation regimen was pursued according to previously published articles. ${ }^{17}$ 
In the hybrid group, an attempt was made to perform closure of the AVF after 6 weeks to 3 months. Phlebography was performed with balloon occlusion of the AVF. When there was slow washout of contrast material ( $>4$ seconds), collaterals were filled, or a stenosis distal to the AVF was found, the AVF closure was not performed. In cases with a dominant inflow vessel without collateral filling, additional stenting into this target vessel was performed.

Consent of individual patients was not obtained as this is not required under Dutch law for retrospective studies. The study was approved by the review board of the medical ethical committee of the AzM/UM (METC 16-4-225).

\section{Statistics}

All continuous data were given as average with standard deviation, except nonnormally distributed data (these were given as median with interquartile range and clearly stated as such). Categorical data were presented as frequencies and percentages. Normally distributed clinical scores were compared using a paired t-test. Non-normally distributed data were compared with a nonparametric Wilcoxon rank test.

Kaplan-Meier survival estimation was used to calculate patency rates. Standard error of the mean values of $>10 \%$ were discarded as being unreliable and were not reported. Patency analyses were based on the total number of extremities treated, in contrast to all other calculations data, which were calculated per patient.

An $\alpha$ of $\leq .05$ was considered statistically significant. All data were analyzed by SPSS Statistics version 23.0 (IBM Corp, Armonk, NY).

\section{Results}

The study included 200 patients with a mean age of 43.2614 .5 (17-81) years. A total of 132 (66\%) were female. In 103 (51\%) patients, a percutaneous procedure was performed to treat post-thrombotic changes up to the CFV confluence; 49 (25\%) patients had a hybrid procedure, and 48 (24\%) were stented because of IVCS. Table 3.1 shows the demographics of all included subjects.

In most cases, the left side was treated $(n=147)$, and the inferior caval vein was additionally stented in 32 patients. The distribution of diseased vein segments is shown in Table 3.2.

In all patients, technical success was achieved, with a maximum of $30 \%$ persistent instent stenosis and brisk flow of contrast material through the stented tract with an arbitrary washout of the contrast material of $<4$ seconds. For the PTS subjects, recanalization was performed to acquire stent placement between healthy vein segments as much as possible. The number of deployed stents differed somewhat, 
depending on the underlying extent of venous obstruction. In those patients with caval treatment, a sinus-XL (OptiMed) was deployed. Table 3.3 shows the distribution of the number of stents used.

Table 3.1 Demographics of all included patients.

\begin{tabular}{|c|c|c|c|c|c|}
\hline & $\begin{array}{l}\text { Hybrid } \\
49(25) \\
\end{array}$ & $\begin{array}{c}\text { Treatment } \\
\text { Percutaneous PTS } \\
103(51) \\
\end{array}$ & $\begin{array}{c}\text { IVCS } \\
48(24)\end{array}$ & $\begin{array}{c}\text { Total } \\
200 / 200\end{array}$ & P-value \\
\hline Age (mean $\pm S D$, min-max) & $40,3(16.5 ; 18-75)$ & $43,7(14.8 ; 17-81)$ & $44,4(11.3 ; 25-77)$ & & 0.31 \\
\hline Females & $31(63)$ & $64(62)$ & $37(77)$ & $132 / 200$ & $0.18^{*}$ \\
\hline Recurrent DVT & $14(29)$ & $27(26)$ & $46(96)$ & $87 / 200$ & $0.17^{*}$ \\
\hline $\mathrm{C}-0$ & $4(8)$ & $8(8)$ & $6(12)$ & & $0.22 *$ \\
\hline $\mathrm{C} 1$ & $8(16)$ & $5(5)$ & $4(8)$ & & \\
\hline $\mathrm{C} 2$ & $8(16)$ & $10(10)$ & $8(17)$ & & \\
\hline $\mathrm{C} 3$ & $17(35)$ & $48(47)$ & $14(29)$ & & \\
\hline $\mathrm{C} 4$ & $7(14)$ & $16(14)$ & $6(12)$ & & \\
\hline $\mathrm{C} 5$ & $2(4)$ & $2(4)$ & $2(4)$ & & \\
\hline C6 & $2(4)$ & $7(7)$ & $6(12)$ & & \\
\hline Treatment side & & & & & $0.11^{*}$ \\
\hline Left & $31(62)$ & $70(67)$ & $46(96)$ & $147 / 239$ & \\
\hline Right & $3(8)$ & $10(10)$ & $0(0)$ & $13 / 239$ & \\
\hline Bilateral & $2(4)$ & $4(4)$ & $2(4)$ & $8 / 239$ & \\
\hline Vena cava and bilateral & $12(24)$ & $19(19)$ & $0(0)$ & $31 / 239$ & \\
\hline Vena cava and unilateral & $1(2)$ & $0(0)$ & $0(0)$ & $1 / 239$ & \\
\hline PE & $6(12)$ & $11(11)$ & $5(10)$ & $22 / 199$ & $0.95^{*}$ \\
\hline Smoking & $3(6)$ & $7(7)$ & $1(2)$ & $11 / 199$ & $0.48^{*}$ \\
\hline Hypertension & $1(2)$ & $1(1)$ & $2(4)$ & $4 / 199$ & $0.43^{*}$ \\
\hline Diabetes & $0(0)$ & $6(6)$ & $0(0)$ & $6 / 199$ & $0.05^{*}$ \\
\hline Cardiac disease & $0(0)$ & $1(1)$ & $0(0)$ & $1 / 199$ & $0.62 *$ \\
\hline Renal disease & $10(20)$ & $17(17)$ & $5(10)$ & $32 / 183$ & $0.07^{*}$ \\
\hline
\end{tabular}

Scores are based in total number of patients. N: number, DVT: deep venous thrombosis, C of CEAP classification, IVCS: iliac vein compression syndrome, PTS: post thrombotic syndrome, PE: pulmonary embolism * A non- parametric test (Kruskall Wallis) for more than 2 independent samples was used for categorical or non- normal distributed variables.

\section{Clinical scores}

All baseline clinical scores were compared with the 12-month follow-up scores, with a median follow-up of 12 (11-12) months. Mean Villalta score decreased from 10.564 .2 $(3-24)$ to $5.363 .8(0-14)$ at the latest follow up $(P<.001)$. The VCSS of the total group decreased by a total of 3 points, ranging from a median score of 7.0 (5-9) at baseline to a median of $4.0(2.5-7.0)$ at latest follow-up $(P<.001)$. Table 3.4 shows the clinical scores at baseline and at the latest follow-up divided per group.

Venous claudication was present in 132 (70\%) patients and remained in 17 patients (13\%) after treatment $(P<.01)$. 
Table 3.2 Distribution of diseased vein segments.

\begin{tabular}{lccc}
\hline & Hybrid & PercutaneousPTS & IVCS \\
\hline LCIV & 0 & 16 & 36 \\
LCFV & 4 & 3 & 0 \\
LEIV+LCIV & 0 & 19 & 10 \\
LCIV+LEIV+LCFV & 22 & 30 & 0 \\
LCIV+LEIV+LCFV+LFPV & 5 & 2 & 0 \\
RCIV & 0 & 0 & 0 \\
RCFV & 1 & 3 & 0 \\
REIV+RCIV & 2 & 4 & 0 \\
RCIV+REIV+RCFV & 0 & 3 & 0 \\
RCIV+REIV+RCFV+RFVP & 0 & 0 & 2 \\
LCIV+RCIV & 2 & 4 & 0 \\
VCI+RCIV & 1 & 0 & 0 \\
VCI+LCIV+RCIV & 4 & 6 & 0 \\
VCI+LCIV+LEIV+RCIV+REIV & 3 & 3 & 0 \\
VCI+LCIV+LEIV+LCFV+RCIV+REIV & 4 & 6 & 0 \\
CCI+LCIV+LEIV+RCIV+REIV+RCFV & 0 & 1 & 0 \\
VCI+LCIV+LEIV+LCFV+RCIV+REIV+RCFV & 1 & 3 & 0 \\
\hline
\end{tabular}

IVC, Inferior vena cava; IVCS, iliac vein compression syndrome; LCFV, left common femoral vein; LCIV, left common iliac vein; LEIV, left external iliac vein; LFPV, left femoral profunda vein; PTS, post-thrombotic syndrome; RCFV, right common femoral vein; RCIV, right common iliac vein; REIV, right external iliac vein; RFVP, right femoral profunda vein.

Table 3.3 Number deployed stents.

\begin{tabular}{llccc}
\hline & & \multicolumn{3}{c}{ Treatment } \\
\cline { 2 - 4 } No of stents & Hybrid & Percutaneous PTS & IVCS \\
& 1 & $5(10)$ & $21(20)$ & $38(79)$ \\
2 & $22(45)$ & $50(48)$ & $6(13)$ \\
3 & $7(15)$ & $11(11)$ & $4(8)$ \\
4 & $2(4)$ & $9(9)$ & \\
5 & $6(12)$ & $5(5)$ & \\
6 & $4(8)$ & $4(4)$ & \\
& 7 & $2(4)$ & $2(2)$ & \\
8 & $1(2)$ & $1(1)$ & \\
\hline
\end{tabular}

$\mathrm{N}$ : number, IVCS: iliac vein compression syndrome, PTS: post thrombotic syndrome. Values are reported as number (\%).

Table 3.4 Clinical scores of included patients before- and after intervention.

\begin{tabular}{lcccc}
\hline & \multicolumn{4}{c}{ Treatment } \\
\cline { 2 - 4 } Score & Hybrid & Percutaneous PTS & IVCS & Based on \\
\hline Villalta scale before intervention & $10,2 \pm 3.8(3-20)$ & $11,2 \pm 4.5(3-24)$ & 135 \\
Villalta scale after intervention & $5,3 \pm 3.4(1-13)$ & $5,2 \pm 3.8(0-14)$ & 93 \\
VCSS before intervention & $7,5(6-10)$ & $7(6-9)$ & $6(4-7)$ & 171 \\
VCSS after intervention & $4(3-9)$ & $5(2-7)$ & $4(2-7)$ & 113 \\
Venous claudication before intervention & $44(90)$ & $66(64)$ & $22(46)$ & 190 \\
Venous claudication after intervention & $4(8)$ & $11(11)$ & $2(4)$ & 135 \\
\hline
\end{tabular}

VCSS: venous clinical severity score, IVCS: iliac vein compression syndrome, PTS: post thrombotic syndrome. Categorical variables are presented as number (\%). Continuous variables are presented as mean \pm standard deviation (minimum-maximum) or median (interquartile range). Scores are based on number of patients. 


\section{Patency scores}

Overall patency scores revealed a primary patency of 68\%, assisted primary patency of $83 \%$, and secondary patency of $90 \%$ at a median of 12 (11-12) months of follow-up (Figure 3.3).

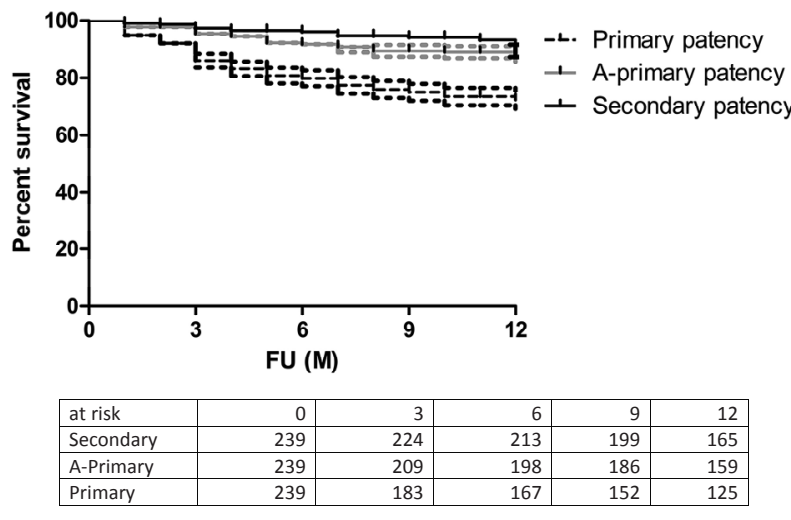

Figure 3.3 Overall Kaplan-Meier curves of stent patency. FU: follow up.

Because a hybrid treatment is more prone to additional interventions, we calculated patency scores for each specific group. A primary patency of $40 \%$, assisted primary patency of $73 \%$, and secondary patency of $76 \%$ were found at a median follow-up of $12(9-12)$ months in the hybrid group.

The percutaneous PTS group revealed patency scores of $71 \%, 82 \%$, and $90 \%$, respectively, at a median followup of 12 (11-12) months. At last follow-up, the IVCS showed a primary patency rate of $92 \%$, assisted primary patency of $96 \%$, and secondary patency of $100 \%$ at a median follow-up of 12 (11-12) months (Figure 3.4.)
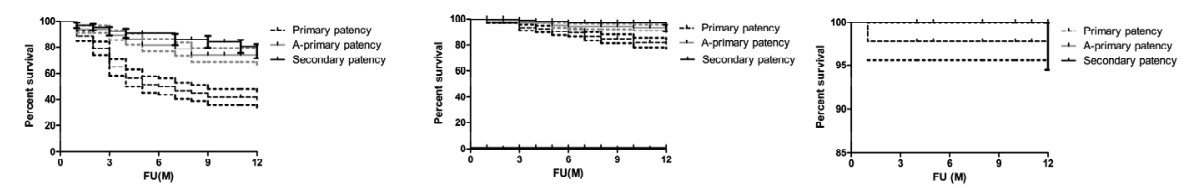

\begin{tabular}{|l|r|r|r|r|r|}
\hline at risk Hybrid & 0 & 3 & 6 & 9 & 12 \\
\hline Secondary & 63 & 64 & 57 & 49 & 43 \\
\hline A-Primary & 63 & 62 & 56 & 47 & 42 \\
\hline Primary & 63 & 55 & 35 & 27 & 23 \\
\hline
\end{tabular}

\begin{tabular}{|l|r|r|r|r|r|}
\hline at risk PTS & 0 & 3 & 6 & 9 & 12 \\
\hline Secondary & 126 & 116 & 112 & 106 & 93 \\
\hline A-Primary & 126 & 107 & 103 & 99 & 86 \\
\hline Primary & 126 & 103 & 96 & 87 & 72 \\
\hline
\end{tabular}

\begin{tabular}{|l|r|r|r|r|r|}
\hline at risk IVCS & 0 & 3 & 6 & 9 & 12 \\
\hline Secondary & 50 & 48 & 46 & 45 & 36 \\
\hline A-Primary & 50 & 45 & 43 & 42 & 33 \\
\hline Primary & 50 & 43 & 41 & 40 & 32 \\
\hline
\end{tabular}

Figure 3.4 Kaplan-Meier curves of stent patency per group. FU: follow up, IVCS: iliac vein compression syndrome, PTS: post thrombotic syndrome. 
Because the occurrence of multiple DVTs could result in higher occlusion rates, the patency scores of PTS patients were analyzed for one or multiple DVTs. In patients with only one DVT in the medical history, this showed patency rates of $65 \%, 85 \%$, and $90 \%$ compared with $60 \%, 75 \%$, and $80 \%$, respectively, for multiple DVTs (Figure 3.5 ).
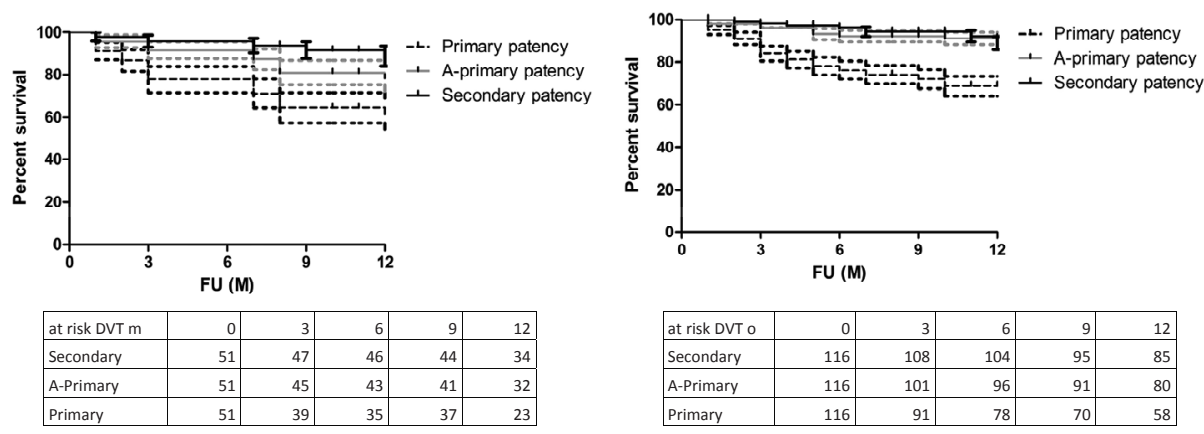

Figure 3.5 Kaplan-Meier curves of stent patency related to the number of deep venous thromboses (DVTs). FU: follow up.

\section{Complications}

Of all included patients, 122 (61\%) did not have a complication during follow-up. Moreover, some patients had multiple complications. The most frequent complications were an in-stent restenosis $(n=23)$ and an occlusion $(n=25)$ of the stented tract. A differentiation was made between a true in-stent restenosis and a recurrent iliac vein compression. When clinical complaints were apparent and the instent restenosis or persistent iliac vein compression was $>50 \%$ (51\%-99\%), this was treated with restenting (meaning stent-in-stent deployment) of the affected segments $(n=16)$. In three patients, a sole PTA was performed to treat a significant in-stent restenosis. In addition, four patients presented with an in-stent restenosis $<50 \%$ (30\%-50\%) and without any complaints, which resulted in conservative management in all of them. If anticoagulation had already been stopped at the time of detection of the in-stent restenosis, it was restarted and a more frequent follow-up was instituted to analyze the degree of in-stent restenosis and clinical complaints. In the aforementioned four patients, the stenosis remained stable during follow-up. In the hybrid group, an attempt was made to close the AVF after 6 weeks to 3 months. A stenosis distal to the AVF was found in 18 patients, of whom 12 received a stent extension into the most dominant inflow vessel. In the remaining six, no additional stenting was possible because of filling of collaterals and related inadequate inflow. When a patient presented with an occlusion after the 3-month follow-up, without the onset or worsening of clinical complaints, no additional treatment was offered $(n=12)$. 
In this case, the patient was advised to use anticoagulation to prevent collateral occlusion. Second, extensive exercise, like climbing the stairs and ambulation as much as possible irrespective of complaints of pain, was recommended to stimulate collateral flow. When an occlusion was noted with the onset of clinical complaints of $<3$ weeks, thrombolysis was offered. Thrombolysis was performed in 10 patients, of whom 3 showed insufficient thrombus resolution. Subsequently, further treatment was stopped, and conservative management was started. In the remaining patients, five had stenting and two received an AVF after successful lysis. At last, three patients had a thrombectomy to treat very early stent occlusion. The number of other complications is presented in Table 3.5. Most notably, there was no death, stent fracture, or angulation, and one patient presented with a contralateral DVT. Other complications included severe menstruation due to anticoagulation therapy, allergy to contrast material, and heparininduced thrombocytopenia.

Table $3.5 \quad$ Number of complications.

\begin{tabular}{lrrrr}
\hline Complication & Hybrid & Percutaneou PTS & IVCS & Total \\
\hline In stent restenosis & $4(2)$ & $19(10)$ & $0(0)$ & $23(12)$ \\
Distal CFV stenosis & $18(9)$ & $0(0)$ & $0(0)$ & $18(9)$ \\
Occlusion & $15(8)$ & $10(5)$ & $0(0)$ & $25(13)$ \\
Tapering & $3(2)$ & $0(0)$ & $0(0)$ & $3(2)$ \\
Persistent external stent compression & $2(1)$ & $8(4)$ & $4(2)$ & $14(7)$ \\
Minor bleeding & $4(2)$ & $0(0)$ & $0(0)$ & $4(2)$ \\
Major bleeding & $2(1)$ & $0(0)$ & $0(0)$ & $2(1)$ \\
Stent migration & $0(0)$ & $1(1)$ & $0(0)$ & $1(1)$ \\
Wound infection & $10(5)$ & $0(0)$ & $0(0)$ & $10(5)$ \\
Wound abcess & $1(1)$ & $0(0)$ & $0(0)$ & $1(1)$ \\
Seroma & $2(1)$ & $0(0)$ & $0(0)$ & $2(1)$ \\
Lymphocele & $4(2)$ & $0(0)$ & $0(0)$ & $4(2)$ \\
Mortality & $0(0)$ & $0(0)$ & $0(0)$ & $0(0)$ \\
Contralateral DVT & $0(0)$ & $1(1)$ & $0(0)$ & $1(1)$ \\
Other & $3(2)$ & $0(0)$ & $2(1)$ & $5(3)$ \\
\hline
\end{tabular}

DVT: deep venous thrombosis, CFV: common femoral vein.

\section{Discussion}

This article describes the results of 200 patients treated with a dedicated venous stent and a median follow-up of 12 months.

For the overall group, primary patency rate was $68 \%$, assisted primary patency was $83 \%$, and secondary patency was $90 \%$. Furthermore, we analyzed whether recurrent DVTs revealed lower patency scores, which currently did not result in a significant difference.

In general, presented differences in PTS and IVCS patency rates were comparable to previous research. ${ }^{3-5,18}$ At large, this can be explained by the fact that venous inflow in 
PTS patients is more compromised because of intraluminal scarring below the FV. Accordingly, it was proven that patients with more extensive post-thrombotic changes show lower patency rates. Because dedicated venous stents have been available for only the past few years, there is currently lack of data for large comparative studies. The primary patency rates in this research were $40 \%$ for hybrid PTS, $71 \%$ for percutaneous PTS, and 92\% for IVCS. These numbers were partly in line with research of Stuck et al., ${ }^{11}$ who showed a primary patency of $83 \%$ for PTS and IVCS patients with the sinus-Obliquus stent (OptiMed) at 10 months of follow-up. However, patency rates of hybrid procedures cannot be compared as those were not performed; they are thought to be lower because of higher probability of hematomas, lymphoceles, and intimal hyperplasia. Moreover, most included patients were younger, healthy women. A second study by O'Sullivan et al. ${ }^{12}$ revealed a clinical patency rate of $85 \%$ with the Zilver Vena stent (Cook Medical, Bloomington, Ind). Compared with the results presented in our study, higher patency numbers are encountered in deploying the sinus-Venous stents. On the contrary, patients analyzed in the Zilver Vena study were included after an acute DVT and malignant venous obstruction and are thus not comparable to our study participants.

Irrespective of the type of stents used, the clinical scores in this study were comparable to previous research. A decrease of VCSS by 3 points has recently been shown in a large cohort with long-term follow-up. ${ }^{19}$ However, the study of Murphy et al. analyzed results in Wallstents (Boston Scientific, Marlborough, Mass), which were initially developed as arterial stents. Moreover, the overall majority of those patients were treated because of caval involvement. Specifically, for the dedicated venous stents, Stuck et al. ${ }^{11}$ revealed outcomes in which the Villalta score decreased by 6 points and the VCSS decreased by 3 points. Moreover, de Wolf et al. ${ }^{10}$ showed a decrease of 6.5 points in Villalta score and a decrease of 3 points in VCSS.

As clinical scores and short-term patency in arterial stent design and dedicated venous design seem to be more or less comparable, a thorough examination should be performed of the occurrence and number of related complications. This specific factor is able to differentiate between multiple stent designs in long-term follow-up. With the introduction of dedicated venous stents, previous research has already demonstrated promising results in reducing the stent-related loss of patency. ${ }^{10}$ In analyzing the number and type of complications, there are several issues specifically described in arterial stent design. Most notably, arterial stents are more rigid, are known to show stent shortening, and may seem to be less able to withstand external compression forces at the iliac part. As a consequence, tapering or kinking of the stent might occur. ${ }^{9}$ The presented outcomes of this study showed tapering in three patients. Moreover, kinking, or a suspicion of kinking, was not seen at all, although this should be mentioned with some prudence as the outcomes of these dedicated venous stents have only short-term follow-up. 
The positioning of stents in the venous system is a challenging procedure in general. This was exemplified by the fact that persistent compression due to arterial overriding was seen 14 times as a learning curve effect. The placement of the stent at the level of the CIV confluence extending into the caval vein is generally not recommended for fear of impairing contralateral outflow, causing an increased risk of contralateral iliac vein thrombosis. ${ }^{20}$ In this cohort, stents were positioned at least at the level of the CIV confluence, generally extending to the spinous process. Eventually, only one patient did develop a DVT, compared with a contralateral DVT incidence of $0 \%$ to $10 \%$ in the Wallstent configuration. ${ }^{18,21}$ This difference in DVT incidence can be explained by the deployment technique but also by the idea that arterial stents usually have a more closed-cell design compared with the open-cell design of the sinus-Venous stent. The smaller interstices of the arterial stents appear to become lined with neointima more often as a stent with an open-cell design. Subsequently, a greater flow is allowed, and contralateral flow will not be jeopardized. ${ }^{21}$

Although favorable results for the sinus-Venous stent have been shown, some limitations should be mentioned. Most notably, long-term outcomes for dedicated venous stents are lacking, and this should be taken into account in prospective research. Overall, to be able to define the iliac confluence more accurately, the use of IVUS might play an important role in future trials. By this means, the number of persistent compressions may be further reduced without giving rise to higher numbers of contralateral DVTs as IVUS is able to calculate the percentage of lumen reduction. ${ }^{21}$ Related to this, the anticoagulation regimen should be standardized, and when coumadin therapy is used, international normalized ratio levels should be analyzed to know whether this is a confounder in stent occlusion or flow impairment. In addition, with the introduction of new oral anticoagulant therapy, a new era of anticoagulation regimen might be opened. Inasmuch as there is no conclusive evidence regarding the type and duration of anticoagulation use after stent placement, we started a trial to analyze the outcomes after coumadin therapy or new oral anticoagulant therapy.

Last, clinical scoring should be standardized for all patients, and the researcher should be obliged to fill out all clinical data at every follow-up moment. Associated with this, the quality of life is probably the most important outcome and should be extensively analyzed to evaluate the clinical effect more precisely. Both general quality of life questionnaires, like the 36-Item Short Form Health Survey (version 2), and diseasespecific outcomes, like the Venous Insufficiency Epidemiological and Economic Study on Quality of Life/Symptoms, should be examined prospectively. Conclusively, when all these factors are standardized, a thorough comparative study can be performed to show the results after stent treatment. 


\section{Conclusion}

Short-term clinical results using the sinus-Venous stent are comparable to previous research. Loss of stent patency due to stent-related issues like kinking or tapering is hardly ever seen in this short-term follow-up. 


\section{References}

1. Kahn SR, Shbaklo H, Lamping DL, Holcroft CA, Shrier I, Miron MJ, et al. Determinants of health-related quality of life during the 2 years following deep vein thrombosis. J Thromb Haemost. 2008;6(7): 1105-12.

2. AbuRahma AF, Robinson PA, Boland JP. Clinical, hemodynamic, and anatomic predictors of long-term outcome of lower extremity venovenous bypasses. J Vasc Surg. 1991;14(5):635-44.

3. Wen-da W, Yu Z, Yue-Xin C. Stenting for chronic obstructive venous disease: A current comprehensive meta-analysis and systematic review. Phlebology. 2016;31(6):376-89.

4. Seager MJ, Busuttil A, Dharmarajah B, Davies AH. Editor's Choice-- A Systematic Review of Endovenous Stenting in Chronic Venous Disease Secondary to Iliac Vein Obstruction. Eur J Vasc Endovasc Surg. 2016;51(1):100-20.

5. Razavi MK, Jaff MR, Miller LE. Safety and Effectiveness of Stent Placement for lliofemoral Venous Outflow Obstruction: Systematic Review and Meta-Analysis. Circ Cardiovasc Interv. 2015;8(10):e002772.

6. Lee BB, Nicolaides AN, Myers K, Meissner M, Kalodiki E, Allegra C, et al. Venous hemodynamic changes in lower limb venous disease: the UIP consensus according to scientific evidence. Int Angiol. 2016;35(3):236-352.

7. Kurstjens RL, de Wolf MA, Konijn HW, Toonder IM, Nelemans PJ, de Graaf R, et al. Intravenous pressure changes in patients with postthrombotic deep venous obstruction: results using a treadmill stress test. J Thromb Haemost. 2016;14(6):1163-70.

8. Jalaie H, Arnoldussen C, Barbati M, Kurstjens R, de Graaf R, Grommes J, et al. What predicts outcome after recanalization of chronic venous obstruction: hemodynamic factors, stent geometry, patient selection, anticoagulation or other factors? Phlebology. 2014;29(1 suppl):97-103.

9. Raju S, Tackett P, Jr., Neglen P. Reinterventions for nonocclusive iliofemoral venous stent malfunctions. J Vasc Surg. 2009;49(2):511-8.

10. de Wolf MA, de Graaf R, Kurstjens RL, Penninx S, Jalaie H, Wittens CH. Short-Term Clinical Experience with a Dedicated Venous Nitinol Stent: Initial Results with the Sinus-Venous Stent. Eur J Vasc Endovasc Surg. 2015;50(4):518-26.

11. Stuck AK, Kunz S, Baumgartner I, Kucher N. Patency and Clinical Outcomes of a Dedicated, SelfExpanding, Hybrid Oblique Stent Used in the Treatment of Common Iliac Vein Compression. J Endovasc Ther. 2017;24(1):159-66.

12. O'Sullivan GJ, Sheehan J, Lohan D, McCann-Brown JA. Iliofemoral venous stenting extending into the femoral region: initial clinical experience with the purpose-designed Zilver Vena stent. J Cardiovasc Surg (Torino). 2013;54(2):255-61.

13. Kurstjens RL, van Vuuren TM, de Wolf MA, de Graaf R, Arnoldussen CW, Wittens $\mathrm{CH}$. Abdominal and pubic collateral veins as indicators of deep venous obstruction. J Vasc Surg Venous Lymphat Disord. 2016;4(4):426-33.

14. Wittens CHA. Invited Commentary. J Vasc Surg Venous Lymphat Disord. 2017;5(5):687-8.

15. Markel A, Meissner M, Manzo RA, Bergelin RO, Strandness DE, Jr. Deep venous thrombosis: rate of spontaneous lysis and thrombus extension. Int Angiol. 2003;22(4):376-82.

16. Jalaie H, Schleimer K, Barbati ME, Gombert A, Grommes J, de Wolf MA, et al. Interventional treatment of postthrombotic syndrome. Gefasschirurgie. 2016;21(Suppl 2):37-44.

17. de Wolf MA, Jalaie H, van Laanen JH, Kurstjens RL, Mensinck MJ, de Geus MJ, et al. Endophlebectomy of the common femoral vein and arteriovenous fistula creation as adjuncts to venous stenting for post-thrombotic syndrome. Br J Surg. 2017;104(6):718-25.

18. Neglen P, Hollis KC, Olivier J, Raju S. Stenting of the venous outflow in chronic venous disease: longterm stent-related outcome, clinical, and hemodynamic result. J Vasc Surg. 2007;46(5):979-90.

19. Murphy EH, Johns B, Varney E, Raju S. Endovascular management of chronic total occlusions of the inferior vena cava and iliac veins. J Vasc Surg Venous Lymphat Disord. 2017;5(1):47-59. 
Patency rates and clinical outcomes in a cohort of 200 patients treated with a dedicated venous stent

20. Caliste XA, Clark AL, Doyle AJ, Cullen JP, Gillespie DL. The incidence of contralateral iliac venous thrombosis after stenting across the iliocaval confluence in patients with acute or chronic venous outflow obstruction. J Vasc Surg Venous Lymphat Disord. 2014;2(3):253-9.

21. Murphy EH, Johns B, Varney E, Buck W, Jayaraj A, Raju S. Deep venous thrombosis associated with caval extension of iliac stents. J Vasc Surg Venous Lymphat Disord. 2017;5(1):8-17. 



\section{Chapter 4}

Stent extension into a single inflow vessel is a valuable option after endophlebectomy 


\section{Abstract}

\section{Background}

Venous stenting with an endophlebectomy and arteriovenous fistula can be performed in patients with extensive post-thrombotic changes. However, these hybrid procedures can induce restenosis, sometimes requiring stent extension, into a single inflow vessel. This study investigates the effectiveness of stenting into a single inflow vessel.

\section{Methods}

All evaluated patients had temporary balloon occlusion of the arteriovenous fistula to evaluate venous flow into the stents. When stent inflow was deemed insufficient, AVF closure was postponed and additional stenting was performed. Patency rates and clinical outcomes were evaluated.

\section{Results}

Twenty-four (38\%) of 64 patients had additional stenting. The primary, assisted primary and secondary patency were $60 \%, 70 \%$ and $70 \%$ respectively. Villalta score reduced by 6.1 points $(P<0.001)$, and venous clinical severity score by 2.7 points $(P=0.034)$.

\section{Conclusion}

Stenting through the femoral confluence into a single inflow vessel is a feasible bailout option if primary hybrid intervention fails with relative high patency rates and clinical improvement. 


\section{Introduction}

Percutaneous transluminal angioplasty (PTA) with venous stenting is a procedure that is gaining increasing interest in the treatment of patients with iliofemoral deep venous obstruction. ${ }^{1}$ This technique shows promising clinical results, excellent patency rates, and low morbidity and mortality. ${ }^{2-7}$ However, successful endovascular treatment in patients with post thrombotic trabeculations and synechiae below the level of the femoral confluence remains a subject of debate and is not routinely performed. ${ }^{8,9}$ Sufficient venous inflow from infra-inguinal vessels (i.e. the femoral vein, FV, and deep femoral vein DFV) plays an important role in patency and clinical improvement. Previous literature findings show reduced patency and subsequently less favorable clinical outcome, whenever inflow is inadequate. ${ }^{9,10}$

However, accurate quantification of inflow and outflow at the level of the common femoral vein (CFV) is currently not possible. Therefore, patient exclusion for an endovascular procedure is usually based on signs of post-thrombotic scar tissue through the CFV confluence, identified on duplex ultrasound (DUS), magnetic resonance venography (MRV) or venography with or without intravascular ultrasound (IVUS). ${ }^{11-14}$ Nevertheless, venous stenting in these patients can still be performed in combination with endophlebectomy and the creation of an arteriovenous fistula (AVF), i.e. a hybrid procedure. ${ }^{14-18}$ Recurrent stenosis in the CFV after hybrid procedures remains an issue in maintaining long-term stent patency and clinical success. Likely, this is a multifactorial process partly due to per-operative vein wall injury, increased thrombogenicity, vein compression due to surrounding scarred tissue and AV-fistula flow. Because of recurrent restenosis, patients require re-intervention and stent extension beyond the endophlebectomy area, typically into a single inflow vessel.

Interestingly, most guidelines discourage stenting below the inguinal ligament, more specifically the femoral confluence. ${ }^{8,19}$ This might partly be because of the presumed risk of stent related complications, i.e. fracture or kinking. Though, first experience with dedicated venous stents highlighted some favorable characteristics, which might eliminate this reason to refrain from distal stenting. ${ }^{14}$ Moreover, after failure of primary surgical treatment, the only available option left may be distal stent extension. Thus, the aim of this study was to investigate whether secondary venous stenting into one inflow vessel caudal to the CFV is feasible and clinically effective after primary hybrid deep venous recanalization fails.

\section{Methods}

We retrospectively analyzed all patients with venous hybrid interventions performed between July 2013 and July 2015. This study included 64 limbs of 61 patients, treated 
primarily by a hybrid procedure at the Maastricht University Medical Centre. All patients were analyzed preoperative by DUS and MRV to locate and assess the severity of obstructions or stenosis. All patients suffered from iliofemoral deep venous obstruction with extension of post-thrombotic vein damage below the femoral confluence and significant complaints interfering with daily activities. Patients with less than 12 months postoperative follow-up were excluded.

Baseline characteristics of all patients were collected and consisted of sex, age, occurrence of deep venous thrombosis (DVT), superficial and deep venous treatment history, Venous Clinical Severity Score (VCSS), Villalta score, and the anamnestic assessment of venous claudication. Venous claudication was scored as being present or absent. Only when patients experienced onset or worsening of pain during ambulation and specifically exercise like climbing the stairs or performing sports it was scored as being present. This pain subsided during rest, especially when sitting or lifting the leg. Venous claudication was referred to as the most important complaint to treat patients.

\section{Hybrid procedure}

Patients were recanalized, received one or multiple stents, an endophlebectomy and an AVF. This procedure has been described in detail before but will be explained briefly. ${ }^{10,20}$ All hybrid procedures were performed under general anesthesia and full anticoagulation. Venous access was acquired through ultrasound-guided puncture of the ipsilateral femoral vein and/or right jugular vein. After successful guidewire and balloon-recanalization, the common femoral vein was opened longitudinal and the intraluminal synechiae were carefully removed. The venotomy was closed by primary closure or patch-plasty and a $6 \mathrm{~mm}$ polytertafluoroethylene AVF was created between the common femoral vein and common femoral artery to reduce the risk of early stent thrombosis.

\section{Hemodynamic evaluation and endovascular stent extension}

The long-term changes caused by an AVF are still unknown. Possible complications might be intimal hyperplasia due to shear stress or cardiac overload. ${ }^{21,22}$ Therefore, all patients were planned for endovascular occlusion of the AVF six weeks to three months after the intervention. Temporary balloon occlusion of the AVF was performed to evaluate venous outflow of the leg. After contralateral common femoral artery (CFA) access, a $5 F 55 \mathrm{~cm}$ sheath was positioned into the ipsilateral CFA, thereafter the AVF loop was catheterized and a $6 \times 20 \mathrm{~mm}$ non-compliant balloon (Powerflex, Cardinal Health/Cordis, USA) was positioned inside the AVF. With the balloon occluding the AVF, an angiography was performed through the ballooncatheter lumen. In case spontaneous venous flow was deemed sufficient, the AVF was 
closed with an Amplatzer plug (St. Jude Medical, Plymouth, MN, USA). Based on our six-year experience, sufficient inflow was scored as an arbitrary cutoff of contrast washout of 4 seconds. When flow was deemed insufficient, the AVF was not closed. An ascending venography, with contrast administration from the foot veins, was performed to identify the major outflow veins and possible alternative routes via collaterals. Additional stenting with a dedicated venous stent (Sinus Venous, Optimed, Optimed $\mathrm{GmbH}$ ) was performed into the vessel below the sapheno-femoral junction with highest quality and flow, i.e. the dominant inflow vessel and the AVF was not closed. This dominant inflow vessel was referred to as the 'target vessel' (Figure 4.1). Patency, complications, VCSS and Villalta score were analyzed 12 months after additional stenting.

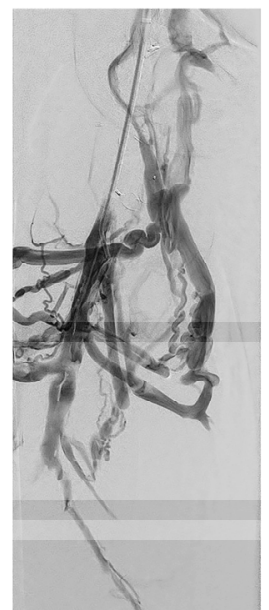

A.

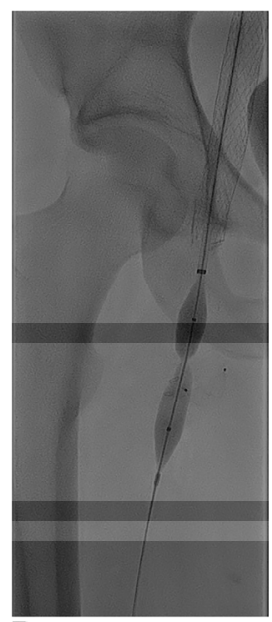

B.

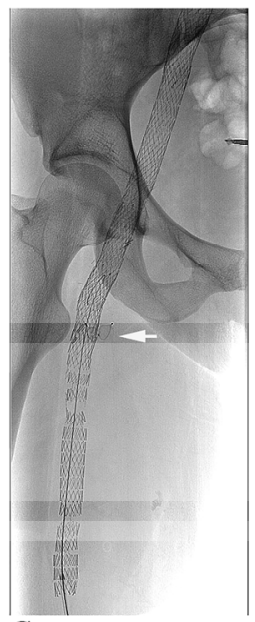

C.

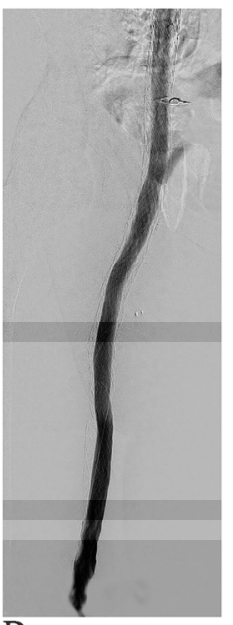

D.

Figure 4.1 Example of recanalization, balloon occlusion and plug occlusion of AV-fistula. (A). After retrograde recanalization from jugular access angiography from the profunda femoral vein shows occlusion of the CFV and an extensive collateral venous network. (B). High-pressure PTA (up to $30 \mathrm{Atm}$.) with a diameter of $12 \mathrm{~mm}$ was necessary to provide enough space for the stent to deployed. (C). Spot image showing a 12x150 mm sinus Venous stent (Optimed GmbH, Ettlingen, Germany) in position. The $8 \mathrm{~mm}$ Amplatzer plug (arrow) placed during an earlier procedure occludes the AV-fistula. Notice the two gaps in the distal stent segment, caused by suboptimal deployment from the jugular approach. (D). However, no residual stenosis was seen and flow was deemed excellent on completion angiography.

\section{Statistics}

All normally distributed continuous data are presented as average with their standard deviation. Non-normally distributed data are presented as median values with inter quartile ranges. Categorical data are shown as frequencies and percentages. A $P \leq .05$ 
was considered statistical significant. Paired T-tests were used to analyze the difference in clinical scoring before and after treatment. Kaplan-Meier survival estimation was used to calculate patency rates. Statistical analysis was performed with IBM SPSS version 23.0 software for Windows (IBM Corporation, Armonk, NY, USA), survival analysis was performed with Graph Pad Prism 5.01 (GraphPad Software, Inc. La Jolla, USA).

\section{Results}

\section{Demographics}

In total, 64 limbs were treated by PTA, stenting and endophlebectomy. In 38 limbs (59.4\%), the balloon occlusion test showed insufficient spontaneous venous inflow. Six out of these 38 limbs did not experience any clinical complaints, while eight had no adequate calibre target vessel suitable for stent extension. In all of them, a conservative management, i.e. compressive stockings and anticoagulation, was maintained. Twenty-four patients (38\%) did experience complaints of heaviness or leg swelling and had one dominant inflow vessel at venography. Additional stenting in the target vessel was successfully performed in all of them. Four of these patients were lost to follow-up and were excluded from further analysis. A total of 20 limbs in 20 patients could be included. Median follow up was 14 months (IQ range 12-21). Demographics of the included patients are shown in Table 4.1. Of all included patients with a median age of 41 years (IQR 26-55), 70\% were female. In 10 patients (50\%), thrombophilia testing was performed of which $60 \%$ had a positive result, mainly indicating Factor $V$ Leiden. Since most patients were already on coumadin therapy when presenting at our outpatient clinic, it was not possible to receive reliable results regarding thrombophilia tests.Moreover, it is not proven that those patients with positive thrombophilia test have higher DVT recurrence rates and as a result no additional tests were performed. ${ }^{23}$ A median stent length of $4.5 \mathrm{~cm}(2.25-10 \mathrm{~cm})$ was deployed in the additional procedure.

\section{Clinical scoring}

Tables 4.1 and 4.2 show clinical scoring before first intervention and after treatment. Statistically significant improvement was seen in Villalta scores and VCSS scores. VCSS was analyzed pre- and post-treatment in 13 patients and showed a mean decrease of 2.7 points $(P=0.034)$. Villalta was scored pre- and postoperative in 13 patients and showed a significant mean decrease of 6.1 points $(P<0.001)$. Venous claudication was scored pre- and postoperative for all patients and was present in 18 (90\%) subjects 
before treatment. After treatment, there was absence of claudication in 17 (90\%) patients.

Table 4.1 Demographics of patients.

\begin{tabular}{|c|c|c|c|}
\hline Demographics & & Percentage & based on $\mathrm{nr}$ patients \\
\hline Age (yr) & & & 20 \\
\hline (median IQR) & $41(26-55)$ & & \\
\hline Females (N) & 14 & 70 & 20 \\
\hline DVT left & 14 & 70 & 20 \\
\hline right & 1 & 5 & 20 \\
\hline bilateral & 5 & 25 & 20 \\
\hline Trombophilia positive & 6 & 60 & 10 \\
\hline VC & 18 & 90 & 20 \\
\hline VCSS score & & & 13 \\
\hline$($ mean $\pm S D(\min -\max )$ & $8,5 \pm 3,2(3-16)$ & & \\
\hline Villalta score (mean \pm SD (min-max) & & & 13 \\
\hline$($ mean $\pm S D(\min -\max )$ & $11 \pm 3,9(4-18)$ & & \\
\hline Abdominal collateral & 17 & 85 & 20 \\
\hline CEAP highest $\mathrm{C}$ & & & 20 \\
\hline $\mathrm{CO}$ & 2 & 10 & \\
\hline $\mathrm{C} 1$ & 5 & 25 & \\
\hline $\mathrm{C} 2$ & 2 & 10 & \\
\hline C3 & 3 & 15 & \\
\hline $\mathrm{C} 4$ & 7 & 35 & \\
\hline C5 & 0 & 0 & \\
\hline $\mathrm{C} 6$ & 1 & 5 & \\
\hline
\end{tabular}

Yr: year, N: number, DVT: deep venous thrombosis, VC: venous claudication, VCSS: venous clinical severity score.

Table 4.2 Post interventional scores.

\begin{tabular}{llccc}
\hline & Outcome & & Based on number patients & $P$-value \\
\hline Side $(\mathrm{N}, \%)$ & left & $17(85 \%)$ & 20 & \\
& $\begin{array}{l}\text { right } \\
\text { bilateral }\end{array}$ & $3(15 \%)$ & & \\
VC & & 0 & 19 & \\
VCSS & & 1 & 13 & 0,034 \\
(mean \pm SD (min-max) & & $5,8 \pm 3,2(0-11)$ & 13 & $<0,001$ \\
Villalta & & & 20 & \\
(mean \pm SD (min-max) & minor & $3(15 \%)$ & & \\
Complication $(\mathrm{N}, \%)$ & major & $7(35 \%)$ & 20 & \\
& & $5(25 \%)$ & & \\
Reintervention $(\mathrm{N}, \%)$ & & & & \\
\hline
\end{tabular}

$\mathrm{N}$ : number, VC: venous claudication, VCSS: venous clinical severity score. VCSS and Villalta post interventional scores are compared to pre interventional scores. A p-value of $\leq .05$ was considered statistical significant. 


\section{Outcome}

Figure 4.2 shows the Kaplan-Meier estimation graph of all treated patients. Patency rates were calculated starting from stenting into the target vessel. Seven (35\%) patients received additional stenting into the FV and 13 (65\%) patients into the DFV.

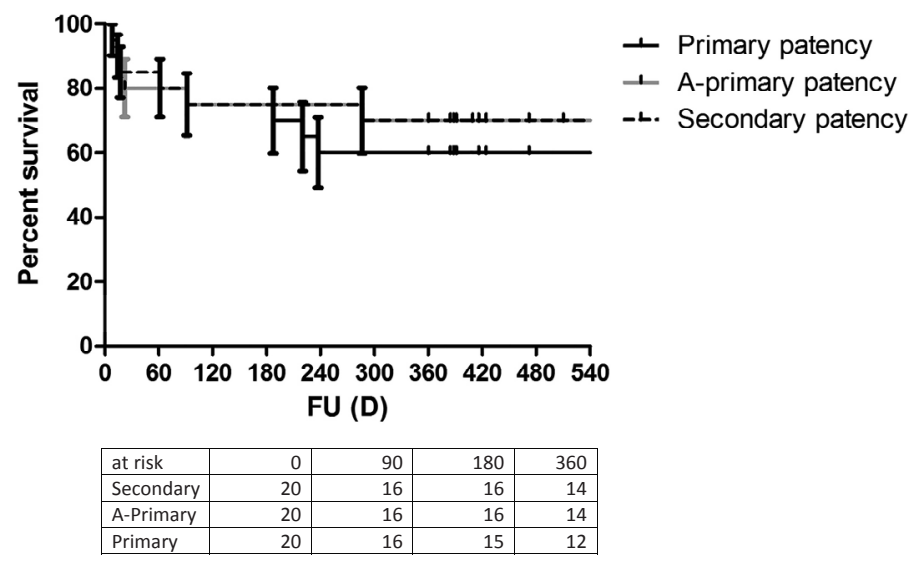

Figure 4.2 Kaplan-Meier survival analysis.

Primary patency of the entire stented tract at 12-month follow-up was $60 \%$. Assisted primary patency and secondary patency were $70 \%$ at 12 -month follow-up. Five patients $(25 \%)$ had additional interventions after stenting into the target vessel. Primary patency was lost in four of them due to stenosis of the iliac tract. Two patients $(10 \%)$ were only treated by PTA and two subjects $(10 \%)$ received new stents of their previously stented iliac tract. One patient (5\%) presented with loss of primary patency due to acute occlusion of the stents for which thrombolysis was started; however, this ultimately did not result in long-term patency.

Seven additional patients (35\%) presented with occlusion of the targeted stented tract which resulted in loss of secondary patency and was scored as a major complication. However, the iliac stents were patent in all of them and thus did not result in loss of secondary patency of the whole stented tract.

One patient (5\%) experienced fever due to superficial cutaneous infection of the puncture location for which oral antibiotic treatment was given, related to as minor complication. Whenever a PTA was deemed necessary, this was defined as minor complication as well. In six patients (30\%), the AVF closed spontaneously, in six patients (30\%) the AVF remained patent so far but will be closed in the follow-up period. In the remaining eight (40\%) patients, the AVF was closed after a median of 160 days. 


\section{Discussion}

In this study, we describe the results of bailout stent extension after failed primary hybrid recanalization of chronic iliofemoral obstructions. The moderate to good outcome of this "single inflow vein stenting" might nuance the debate about stenting below the CFV. Although some guidelines mention that stenting below the inguinal ligament should be avoided, general experience described in recent literature supports stenting into the CFV when necessary, since the main goal is to completely treat all diseased vein segments proximal to the CFV confluence. In contrast, primary stenting into one inflow vessel distal to the CFV confluence, i.e. DFV or FV, is currently not supported in the literature. Therefore, we usually offer a hybrid procedure to patients with post-thrombotic trabeculation distal to the level of the CFV confluence. This hybrid procedure, including percutaneous stenting and additional endophlebectomy, has shown favourable secondary patency between $72 \%$ and $90 \%$ and clinical decrease of Villalta with a median of 7 points. ${ }^{16,17,20,24}$ However, patient selection for this procedure remains an important topic since complications like lymphorrhoea and wound infections can occur in an amount of subjects. This should be thoroughly discussed with a patient before the first intervention in which benefits of the intervention and morbidity after intervention should be weighted against each other. Moreover, in patients with hybrid interventions, recurrent obstruction at the level of the CFV is an issue in which the only valuable option to maintain stent patency is to perform stent extension into an adequate inflow vein. We demonstrated that stenting into such a target vessel below the femoral confluence resulted in a secondary patency of $70 \%$ after 12 months. This seems surprisingly high for a bailout procedure in patients in which the primary hybrid intervention failed, which in fact, shows a lower overall patency.

Nevertheless, there is no accurate data to which our results can be compared, since primary stenting into a single vein below the CFV confluence has not been described before. Therefore, our intention was to describe the technical aspects of this endovascular solution and assess feasibility and effectiveness treating recurrent obstruction after endophlebectomy of the CFV.

We critically evaluated the cases in which patency was lost after stent extension. In all of them, stent related complications were ruled out. The possibility of kinking, residual or recurrent stent compression was eliminated by evaluating the duplex images. The fact that dedicated venous stents were used with high flexibility and radial forces might be beneficial in this case. Postoperative anticoagulation might have been a critical factor. Although we have a standard postoperative regimen of six months coumadin therapy with a target INR level of 3-4, anticoagulation therapy is monitored and regulated by a separate National institution and it cannot be completely ruled out that suboptimal anticoagulation therapy influenced primary and secondary patency. 
Another reason for stent occlusion might be impaired flow through the stented segments, caused by insufficient size or quality of the inflow vessel. In many patients with extended post-thrombotic disease, significant blood volume is forced through multiple competitive collateral veins. Therefore, less blood is reaching the CFV through the diseased FV and DFV segments. Subsequently, this altered venous outflow tract is not reaching the stents, reducing the amount of blood flow needed to maintain stent patency.

The main reason patients undergo deep venous reconstruction is the relief of complaints. The clinical success of treatment is specifically evaluated by comparing VCSS, Villalta and venous claudication scores before and after treatment. Villalta scale and venous claudication significantly improved after stent extension into a single inflow vein. More importantly, patients with stent re-occlusion did not experience worsening of their complaints compared to the situation before stent extension, expressed in Villalta and VCSS scores and more specifically the venous claudication score. This might be explained by the pre-existent collateral network, which is unlikely to be harmed by stent extension. Moreover, did all patients with stent occlusion have a patent iliac stent tract. In our experience, occlusion of the iliac tract can result in more debilitating complaints than occlusion in the distal part of the leg. This could have been found due to the lesser capability of collateral formation in the more central veins compared to the veins in the lower extremity.

As a possible limitation of this study it should be mentioned that the follow-up period is relatively short which could result in lower patency rates on the long term. With the current possibility of IVUS imaging, this could, however, provide benefit in detecting stenosis at an earlier term. With a follow-up protocol of 10 years minimum, long-term results can be provided in future research.

Future discussions might further address whether primary stenting into a single inflow vein could be a first choice treatment instead of an endophlebectomy. Apart from patency, the main advantage of this endovascular strategy would be the reduction of surgery related complications like wound infections and lymphorroea. Previous research from our group demonstrated the occurrence of lymphorroea or wound infections in about one-third of all patients with endophlebectomy and AV fistula. Due to this high possible morbidity, it would be a great advantage to opt for primary stent placement into a target vessel in a selected group of patients. Nevertheless, it seems unlikely that all patients can be treated without an endophlebectomy, since stenting into a relatively clean vein segment is a very important factor in the management of deep venous obstructions. ${ }^{8,9}$ In patients with extensive post-thrombotic changes in all potential target vessels, endophlebectomy might remain the best option. Furthermore, the high success rate of stent extension as a bailout procedure cannot be extrapolated to primary stenting into one inflow vein. The iliofemoral stents placed during the hybrid procedure are more or less incorporated in the vein wall at the time of stent extension. This might have an effect on thrombogenicity compared to primary 
stenting. Primary stenting into a dominant inflow vessel may have a higher thrombosis risk due to a relatively low flow rate and an extremely long stent length. Beyond assumptions, it would be sensible to prospectively compare the best possible endovascular option with a hybrid intervention in selected comparable patients.

\section{Conclusion}

Stenting below the femoral confluence into a single inflow vessel is a feasible bailout option if primary hybrid intervention fails. Future research should determine if a sole endovascular procedure, with primary stenting into a single inflow vessel below the femoral confluence might be a valuable alternative for CFV endophlebectomy. 


\section{References}

1. Hartung O, Loundou AD, Barthelemy P, Arnoux D, Boufi M, Alimi YS. Endovascular management of chronic disabling ilio-caval obstructive lesions: long-term results. Eur J Vasc Endovasc Surg. 2009; 38(1): 18-24.

2. Alhalbouni S, Hingorani A, Shiferson A, Gopal K, Jung D, Novak D, et al. Iliac-femoral venous stenting for lower extremity venous stasis symptoms. Ann Vasc Surg. 2012;26(2):185-9.

3. Friedrich de Wolf MA, Arnoldussen CW, Grommes J, Hsien SG, Nelemans PJ, de Haan MW, et al. Minimally invasive treatment of chronic iliofemoral venous occlusive disease. J Vasc Surg Venous Lymphat Disord. 2013;1(2):146-53.

4. Hartung O, Benmiloud F, Barthelemy P, Dubuc M, Boufi M, Alimi YS. Late results of surgical venous thrombectomy with iliocaval stenting. J Vasc Surg. 2008;47(2):381-7.

5. Neglen P, Hollis KC, Olivier J, Raju S. Stenting of the venous outflow in chronic venous disease: longterm stent-related outcome, clinical, and hemodynamic result. J Vasc Surg. 2007;46(5):979-90.

6. Rosales A, Sandbaek G, Jorgensen JJ. Stenting for chronic post-thrombotic vena cava and iliofemoral venous occlusions: mid-term patency and clinical outcome. Eur J Vasc Endovasc Surg. 2010;40(2): 234-40.

7. Titus JM, Moise MA, Bena J, Lyden SP, Clair DG. Iliofemoral stenting for venous occlusive disease. J Vasc Surg. 2011;53(3):706-12.

8. Mahnken AH, Thomson K, de Haan M, O'Sullivan GJ. CIRSE standards of practice guidelines on iliocaval stenting. Cardiovasc Intervent Radiol. 2014;37(4):889-97.

9. Neglen P, Tackett TP, Jr., Raju S. Venous stenting across the inguinal ligament. J Vasc Surg. 2008; 48(5):1255-61.

10. Kurstjens RL, de Graaf R, Barbati ME, de Wolf MA, van Laanen JH, Wittens $\mathrm{CH}$, et al. Arteriovenous fistula geometry in hybrid recanalisation of post-thrombotic venous obstruction. Phlebology. 2015; 30(1 Suppl):42-9.

11. Arnoldussen $\mathrm{CW}$, Toonder I, Wittens $\mathrm{CH}$. A novel scoring system for lower-extremity venous pathology analysed using magnetic resonance venography and duplex ultrasound. Phlebology. 2012; 27 Suppl 1:163-70.

12. Cavezzi A, Labropoulos N, Partsch H, Ricci S, Caggiati A, Myers K, et al. Duplex ultrasound investigation of the veins in chronic venous disease of the lower limbs--UIP consensus document. Part II. Anatomy. Eur J Vasc Endovasc Surg. 2006;31(3):288-99.

13. Coleridge-Smith P, Labropoulos N, Partsch H, Myers K, Nicolaides A, Cavezzi A. Duplex ultrasound investigation of the veins in chronic venous disease of the lower limbs--UIP consensus document. Part I. Basic principles. Eur J Vasc Endovasc Surg. 2006;31(1):83-92.

14. de Wolf MA, Arnoldussen $\mathrm{CW}$, Wittens $\mathrm{CH}$. Indications for endophlebectomy and/or arteriovenous fistula after stenting. Phlebology. 2013;28 Suppl 1:123-8.

15. Comerota AJ, Grewal NK, Thakur S, Assi Z. Endovenectomy of the common femoral vein and intraoperative iliac vein recanalization for chronic iliofemoral venous occlusion. J Vasc Surg. 2010; 52(1):243-7.

16. Garg N, Gloviczki P, Karimi KM, Duncan AA, Bjarnason H, Kalra M, et al. Factors affecting outcome of open and hybrid reconstructions for nonmalignant obstruction of iliofemoral veins and inferior vena cava. J Vasc Surg. 2011;53(2):383-93.

17. Puggioni A, Kistner RL, Eklof B, Lurie F. Surgical disobliteration of postthrombotic deep veins-endophlebectomy--is feasible. J Vasc Surg. 2004;39(5):1048-52; discussion 52.

18. Vogel D, Comerota AJ, Al-Jabouri M, Assi ZI. Common femoral endovenectomy with iliocaval endoluminal recanalization improves symptoms and quality of life in patients with postthrombotic iliofemoral obstruction. J Vasc Surg. 2012;55(1):129-35.

19. Bjarnason H. Handbook of Angioplasty and Stenting Procedures, Techniques in Interventional Radiology- Venoplasty and Stenting. 2010:303-15. 
20. de Wolf MA, Jalaie H, van Laanen JH, Kurstjens RL, Mensinck MJ, de Geus MJ, et al. Endophlebectomy of the common femoral vein and arteriovenous fistula creation as adjuncts to venous stenting for post-thrombotic syndrome. Br J Surg. 2017;104(6):718-25.

21. Hammes M. Hemodynamic and biologic determinates of arteriovenous fistula outcomes in renal failure patients. Biomed Res Int. 2015;2015:171674.

22. Roy-Chaudhury $P$, Arend L, Zhang J, Krishnamoorthy M, Wang $Y$, Banerjee R, et al. Neointimal hyperplasia in early arteriovenous fistula failure. Am J Kidney Dis. 2007;50(5):782-90.

23. Baglin $\mathrm{T}$, Luddington $\mathrm{R}$, Brown $\mathrm{K}$, Baglin $\mathrm{C}$. Incidence of recurrent venous thromboembolism in relation to clinical and thrombophilic risk factors: prospective cohort study. Lancet. 2003; 362(9383):523-6.

24. Jalaie H, Arnoldussen C, Barbati M, Kurstjens R, de Graaf R, Grommes J, et al. What predicts outcome after recanalization of chronic venous obstruction: hemodynamic factors, stent geometry, patient selection, anticoagulation or other factors? Phlebology. 2014;29(1 suppl):97-103. 



\section{Chapter 5}

Stent extension below the common femoral vein in extensive chronic iliofemoral venous obstructions 


\section{Abstract}

\section{Purpose}

To analyze whether primary venous stent placement into 1 dominant inflow vein peripheral to the common femoral vein (CFV) confluence is feasible.

\section{Materials and methods}

Retrospective review was performed of 14 consecutive patients who underwent primary venous stent placement into veins peripheral to the CFV between 2013 and 2016. Mean patient age was 49 years; 6 (43\%) patients were women. All patients had successful deep venous stent placement with brisk contrast flow through the stent. Patients had primary percutaneous stent placement when postthrombotic changes extended peripherally to the femoral confluence but a trabeculation-free area in the deep femoral vein (DFV) could be identified. Based on imaging findings, the DFV had to be considered the prominent inflow vein with normal anatomy. Femoral vein, DFV, and collateral inflow were minimally impaired owing to postthrombotic scarring or trabeculations.

\section{Results}

Primary, assisted primary, and secondary patency rates were $92 \%$ at a median follow-up of $481 \mathrm{~d}$ (range, 411-792 d). Venous Clinical Severity Score decreased from a mean of 8.9 to 6.4 $(P=.03)$. The Villalta scale decreased from a mean of 11.7 to $4.3(P=.003)$. Before intervention, venous claudication was present in $92 \%$ and remained in $38 \%$ after intervention $(P=.016)$.

\section{Conclusions}

Stent placement through the femoral confluence into a dominant inflow vein is a promising option in a carefully selected group of patients. 


\section{Introduction}

Postthrombotic syndrome is a debilitating, frequently longterm complication following a deep vein thrombosis. ${ }^{1,2}$ Treatment of postthrombotic obstruction peripheral to the inguinal ligament and involving the femoral confluence in particular, remains a challenge and is not specifically addressed by most guidelines. ${ }^{3}$ As venous inflow may be insufficient to support stent patency in such cases, a hybrid procedure combining endophlebectomy and arteriovenous fistula (AVF) might be a good alternative to an endovascular procedure. Small cohort series have shown favorable results in patients for whom alternative interventional treatment options were deemed suboptimal; ${ }^{4-7}$ however, the number of complications and reinterventions is considerably higher compared with percutaneous treatment alone. First, owing to the open surgical procedure, wound infections and wound dehiscence can occur. Second, compression from hematomas or lymphoceles in the recently operated common femoral vein (CFV) may negatively affect primary patency. Third, the AVF may potentially induce intimal hyperplasia and in-stent stenosis. ${ }^{4,8}$

It seems ideal to keep open surgical therapy to a minimum and choose a minimally invasive intervention when possible. The main challenge is to select patients in whom a nonfibrotic venous segment can be found for stent placement, a practice that adheres to the widely held belief that stent placement should be performed "from healthy to healthy". 9 The potential of this strategy is supported by earlier results showing bailout stent placement into a single inflow vein to be a feasible option after primary hybrid surgical intervention with secondary patency rates of $70 \%{ }^{10}$ The aim of this study was to analyze whether primary venous stent placement into a dominant inflow vein peripheral to the CFV confluence is feasible in selected cases.

\section{Materials and methods}

This study was approved by the local institutional review board and ethical committee. Between July 2013 and January 2016, 79 patients were treated for postthrombotic changes extending peripherally to the CFV. All patients underwent successful stent placement. In 65 (82\%) patients, a hybrid procedure combining endophlebectomy and AVF was performed to increase inflow into the stents. In 14 (18\%) patients, primary stent placement peripheral to the femoral confluence was performed, and these patients were included in this study.

All consecutively included patients presented with substantial clinical symptoms, such as pain, edema, and venous claudication, that interfered with daily activities. In patients with minimal clinical signs, the presence of venous claudication was the main indication for treatment. In all patients, both duplex ultrasound (US) and magnetic resonance (MR) venography revealed signs of postthrombotic stenosis, 
trabeculations, or scarring. Baseline characteristics, such as age, sex, side of treatment, and clinical scores, were retrospectively analyzed and entered into a database. Patency rates and complications were analyzed for all included patients.

\section{Selection criteria for primary stent placement peripheral to the femoral confluence}

All patients were treated by an interventional radiologist. Treatment planning was based on a multidisciplinary consultation between the radiologist and 2 vascular surgeons. Before intervention, eligible patients underwent evaluation of postthrombotic obstructions by duplex US and MR venography. Based on the extensiveness of postthrombotic scarring and collateral venous flow, patients were treated with either a hybrid surgical intervention or percutaneous stent placement. When obstruction extended peripherally to the femoral confluence, especially when the CFV was occluded or when there was a risk that stent placement could displace intraluminal tissue and compromise the inflow of the femoral vein (FV) or deep femoral vein (DFV), a hybrid procedure with endophlebectomy and AVF was preferred. Also, when the orifices of the FV and/or the DFV were occluded, patients were included in the hybrid surgical treatment group. These patients were excluded from analysis in this study, as the goal was to analyze the outcome of stent placement peripheral to the femoral confluence into 1 inflow vein.

Patients had primary percutaneous stent placement when postthrombotic changes extended peripherally to the femoral confluence but a trabeculation-free area in the DFV could be identified. Based on imaging findings, the DFV had to be considered the prominent inflow vein with normal anatomy. Furthermore, the inflow from the FV, DFV, and collaterals needed to be minimally impaired owing to postthrombotic scarring or trabeculations (Table 5.1).

The demographics of all included patients are shown in Table 5.2. Mean patient age was 49 years, and $43 \%$ of patients were women. All patients had successful deep venous stent placement with brisk contrast flow through the stent without significant stenosis.

\section{Intervention}

After traversing the postthrombotic trabeculations, transluminal angioplasty was performed generally using a 16- $\mathrm{mm}$ balloon for the common iliac vein, a 14-mm balloon for the external iliac vein, and 12-mm balloon for the FV and/or DFV. Thereafter, stents were deployed, and dilation of the stents was performed after deployment. A $14-\mathrm{mm}$ or $16-\mathrm{mm}$ sinus-Obliquus stent (Optimed $\mathrm{GmbH}$, Ettlingen, Germany) was placed in bilateral iliac veins. A $14-\mathrm{mm}$ or $16-\mathrm{mm}$ sinus-Venous stent (Optimed $\mathrm{GmbH}$ ) was placed in unilateral iliac veins. The stents were extended into 
the dominant inflow vein with highest quality and flow. The diameter of this target vein needed to be at least $10 \mathrm{~mm}$ to enable deploying a 10 - to $12-\mathrm{mm}$ stent. This was tested by balloon occlusion of the CFV with administration of contrast medium directly into the DFV (Figure 5.1a-d). Sufficient inflow was deemed as an arbitrary cutoff of contrast washout of 4 seconds.

Table 5.1 Distribution of postthrombotic changes before treatment in treated veins.

\begin{tabular}{lcccc}
\hline Number & Iliac vein & Common femoral vein & Femoral vein & Deep femoral vein \\
\hline 1 & $50 \%-90 \%$ & $<10 \%$ & $50 \%-90 \%$ & $10 \%-50 \%$ \\
2 & $50 \%-90 \%$ & $10 \%-50 \%$ & $10 \%-50 \%$ & $10 \%-50 \%$ \\
3 & $10 \%-50 \%$ & $10 \%-50 \%$ & $10 \%-50 \%$ & $10 \%-50 \%$ \\
4 & $<10 \%$ & $<10 \%$ & $10 \%-50 \%$ & $10 \%-50 \%$ \\
5 & $50 \%-90 \%$ & $10 \%-50 \%$ & $10 \%-50 \%$ & $10 \%-50 \%$ \\
6 & $<10 \%$ & $<10 \%$ & $<10 \%$ & $<10 \%$ \\
7 & $50 \%-90 \%$ & $10 \%-50 \%$ & $10 \%-50 \%$ & $10 \%-50 \%$ \\
8 & $50 \%-90 \%$ & $50 \%-90 \%$ & $10 \%-50 \%$ & $0 \%$ \\
9 & $50 \%-90 \%$ & $50 \%-90 \%$ & $0 \%$ & $0 \%$ \\
10 & $10 \%-50 \%$ & $10 \%-50 \%$ & $50 \%-90 \%$ & $0 \%$ \\
11 & $50 \%-90 \%$ & $50 \%-90 \%$ & $50 \%-90 \%$ & $0 \%$ \\
12 & $50 \%-90 \%$ & $10 \%-50 \%$ & $10 \%-50 \%$ & $<10 \%$ \\
13 & $50 \%-90 \%$ & $10 \%-50 \%$ & $10 \%-50 \%$ & $<10 \%$ \\
14 & $50 \%-90 \%$ & $10 \%-50 \%$ & $<10 \%$ & $<10 \%$ \\
\hline
\end{tabular}

Table 5.2 Demographics.

\begin{tabular}{lc}
\hline Characteristics & Value \\
\hline Age, $y$, mean \pm SD (minimum-maximum & $49 \pm 12(33-73)$ \\
Sex & $6(43)$ \\
Female, $\mathrm{n}(\%)$ & $8(57)$ \\
Male, $\mathrm{n}(\%)$ & \\
Side target vessel & $8(57)$ \\
Left, $\mathrm{n}(\%$ ( & $6(43)$ \\
Right, $\mathrm{n}(\%)$ & \\
CEAP, highest C, $\mathrm{n}$ & 0 \\
C0 & 1 \\
C1 & 1 \\
C2 & 7 \\
C3 & 2 \\
C4 & 1 \\
C5 & 2 \\
C6 & 2 \\
\hline
\end{tabular}

$\mathrm{Y}$ : year; $\mathrm{n}$ : number; C: clinical

\section{Follow-up}

After intervention, intermittent pneumatic compression stockings were applied when the patient was immobilized. All patients were evaluated before hospital discharge and at 2 weeks, 6 weeks, 3 months, 6 months, and 12 months after intervention. 
During every follow-up visit, stent patency was evaluated, and clinical outcomes were determined based on the Venous Clinical Severity Score, Villalta scale, and presence or absence of venous claudication. Stent patency was evaluated by duplex US and documented as patent (showing any blood flow through the stents) or occluded. Stenosis was documented based on the percentage of lumen reduction. When the detected stenosis was $>50 \%$ and clinical symptoms recurred or worsened over time, an additional treatment was discussed.

After initial treatment, all patients received low-molecularweight heparin and warfarin therapy simultaneously until an international normalized ratio of 3-4 was reached. Warfarin was continued for a minimum of 6 months and longer when stent stenosis occurred or when the medical history required prolonged therapy. The evaluation of international normalized ratio levels was standardized and regulated by separate institutes (all according to Dutch guidelines). Antiplatelet therapy was not used in any of the patients.
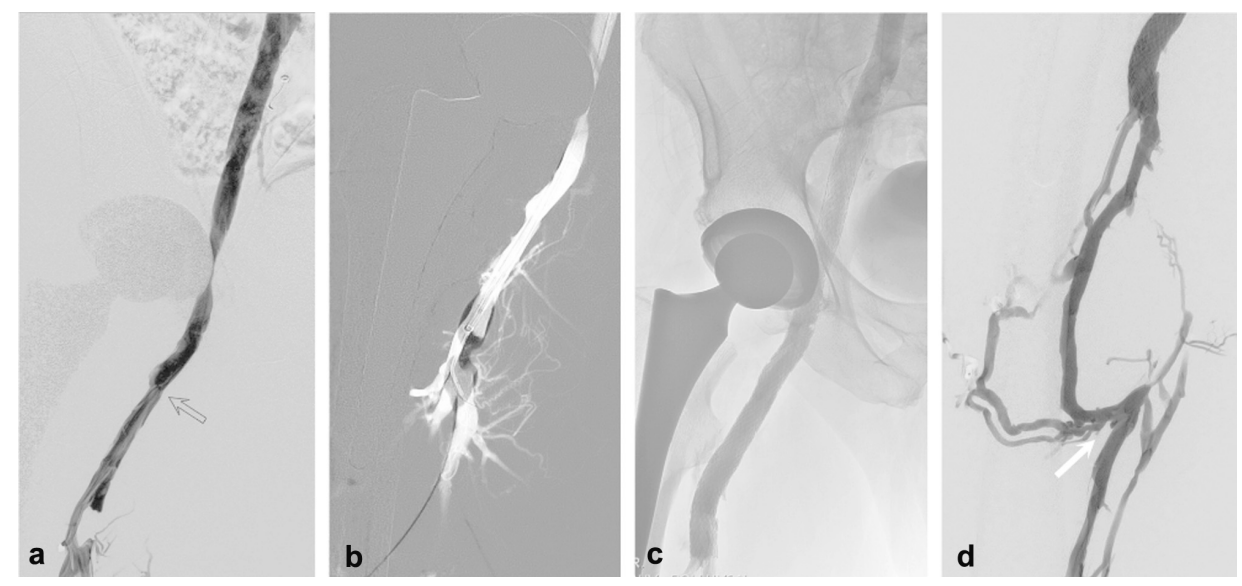

Figure 5.1 (a) Venography after stent placement from the right iliac veins into the common femoral vein. Note the significant stenosis in deep femoral vein outflow (arrow). (b) PTA with a $12 \times 40 \mathrm{~mm}$ balloon shows a waist in the balloon pointing to a fibrotic postthrombotic lesion. (c) Venography performed after stent placement from the right popliteal vein shows exclusive flow through the iliofemoral stents. No collaterals are filling, which suggests unobstructed venous outflow. (d) Venography from the popliteal vein showing occlusion of the femoral vein (arrow), with preferential flow through collaterals and deep femoral vein into the iliofemoral stents.

\section{Statistics}

All normally distributed continuous data were reported as average with SD. Nonnormally distributed data were reported as median values with interquartile ranges. 
Categorical data were reported as frequencies and percentages. An $\alpha<.05$ was considered statistically significant. Paired t tests were used to analyze the difference in clinical scoring before and after treatment. The McNemar test was used to compare binary outcomes. Kaplan-Meier survival estimation was used to calculate patency rates. SEM $>10 \%$ was regarded as unreliable, and figures were discarded this point. Patency rates were calculated per leg. Statistical analysis was performed with IBM SPSS Version 23.0 (IBM Corp, Armonk, New York), and survival analysis was performed with GraphPad Prism 5.01 (GraphPad Software, La Jolla, California).

\section{Results}

\section{Intervention}

Stent placement was performed in all patients with the DFV as the target vein. One patient $(7 \%)$ received stents after thrombolysis for an acute deep vein thrombosis and postthrombotic chronic disease more peripherally. A median of 2.5 stents (range, 1-7 stents) was used, and stents were inserted in both legs up to the iliac veins in 4 patients. In all patients, a stent was inserted in only 1 leg peripherally to the CFV. A sinus-Venous stent was placed in the target vein. The diameter and length of deployed stents in this target area were sized according to the vein quality and diameter with a variation between $10 \mathrm{~mm}$ and $14 \mathrm{~mm}$. The median length of stent extension was $105 \mathrm{~mm}$ (range, 20-150 mm).

\section{Outcomes}

Clinical outcomes were scored at the outpatient clinic by physicians and researchers who had not performed the interventional therapy. Venous Clinical Severity Score was obtained before intervention for 13 patients (mean score $8.9 \pm 4.4$; range, 3-20). Venous Clinical Severity Score after intervention decreased significantly (mean score $6.4 \pm 3.5$; range, $1-12 ; P=.03$ ). The Villalta scale was evaluated in 13 patients before and after intervention. A mean decrease of 5.7 points was seen with a mean score of $11.7 \pm 4.3$ (range, 5-20) before intervention and $6.0 \pm 3.6$ (range, 0-13) after intervention $(P=.003)$. Data regarding venous claudication before intervention were available in 13 patients, of whom 12 had symptoms indicative of claudication (92\%). After treatment, claudication subsided in 8 of the patients, for an incidence of $38 \%$ $(P=.016)$.

Figure 5.2 shows the Kaplan-Meier survival estimates of all patients. A combined primary patency, assisted primary patency, and secondary patency of $92 \%$ is shown with a median follow-up of 481 days (range, 411-792 d). During the entire follow-up period, 1 patient (7\%) experienced stent occlusion. In this patient, percutaneous 
transluminal angioplasty (PTA) and a second stent placement were performed, which resulted in a patent stent for 11 more months, which occluded thereafter. Besides the thrombotic stent occlusion of this patient, 3 more patients experienced a complication at some point during the follow-up period. In all 3 patients, an external compression of $30 \%$ in the left common iliac vein was found without any clinical symptoms. For this reason and owing to the lumen reduction without progression over time, no additional treatment was discussed. No bleeding complications occurred during treatment or follow-up.
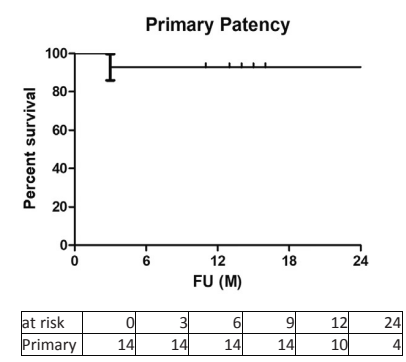
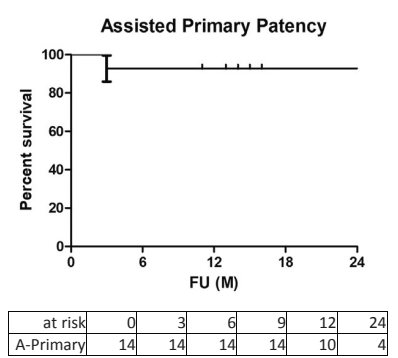
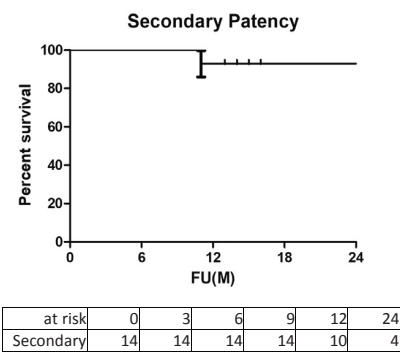

Figure 5.2 Kaplan-Meier curves of stent patency. FU, follow-up.

\section{Discussion}

This study investigated the feasibility of primary stent placement in a dominant inflow vein peripheral to the femoral confluence. Clinical outcomes and patency rates were promising and at least comparable to hybrid surgical procedures and other treatment alternatives. ${ }^{8}$ In the past, extensive open bypass surgery was performed with poor to moderate patency rates and clinical outcomes. ${ }^{11}$ Minimally invasive PTA and stent placement were subsequently introduced and replaced these major open surgical reconstructions. With refinement of angiography techniques, the addition of intravascular US, and the introduction of dedicated venous stents, the overall treatment of patients with postthrombotic obstructions has improved. However, it has repeatedly been reported that postthrombotic changes peripherally to the CFV are challenging problems for endovascular options; consequently, stent patency is considerably adversely affected. ${ }^{3,12}$ A reported alternative treatment is surgical endophlebectomy and AVF creation, which is a hybrid procedure, with moderate patency rates and clinical results. ${ }^{4,8}$ Owing to the high morbidity related to this open surgical hybrid intervention, it is not a widely accepted strategy; minimally invasive interventions, although far from perfect, are still preferred.

Thus far, stent placement peripheral to the femoral confluence has been discouraged, and large comparative studies between open surgical hybrid treatment and 
endovascular treatment options are not available. However, Neglen et al. ${ }^{9}$ described stent placement peripheral to the inguinal ligament as a promising option; this treatment should not be avoided for fear of stent fractures. The most important avoided commandment was to cover the obstructed area, as stent patency is mainly related to the type of pathology. Falcoz et al. ${ }^{13}$ published their single-center outcomes in endovascularly treated patients with postthrombotic syndrome; 8 of 21 patients had stent placement in the FV. Although 2 of 21 patients presented with an occlusion during the follow-up period, the researchers did not mention which vein area was occluded. Furthermore, 19 stents were patent at the end of follow-up, which indicated the feasibility of stent placement peripheral to the femoral confluence. Raju et al. ${ }^{14}$ emphasized this by noting in unpublished data that stent placement in the DFV could be a good option.

The results of this study stress the possibility of performing stent placement in a dominant inflow vein in selected cases, thereby avoiding open surgery-elated complications. Although, to our knowledge, there are no previous studies investigating stent placement in a dominant inflow vein peripheral to the femoral confluence, a secondary patency of $92 \%$ seems more than reasonable considering the challenging patient group treated. Therefore, with a patency up to $72 \%$ at the 36-month follow-up, the extended endovascular approach seems to be a promising alternative compared with hybrid surgical interventions. ${ }^{8}$ Moreover, surgery-related complications are prevented, and the hospital stay is shortened. Additionally, patients do not need an elective procedure to close the AVF. With acceptable patency rates, a lower complication risk for the patient, and probably lower overall costs, the potential benefits are obvious.

It must be noted that stent extension into a dominant inflow vein was performed in a selected group of patients. All included patients had an adequate DFV diameter and adequate vein quality without significant trabeculations. A hybrid surgical procedure still seems to be the best option if all inflow veins are scarred and spontaneous inflow is largely hampered. Compared with the hybrid surgical procedure, clinical scores improved to a lesser extent in patients with primary stent extension: $38 \%$ of patients with venous claudication did not improve compared with $17 \%$ of patients undergoing the hybrid procedure. ${ }^{8}$ This lower improvement with primary stent extension could be explained by the fact that the quantification of blood flow through the stents remains difficult. With the existence of collateral flow, it is possible that an increased amount of blood will tend to flow through collaterals instead of the long area of the vein with a stent. Moreover, a large number of patients have pre-existing peripheral disease, which is currently not re-evaluated after stent placement. To exclude this as a confounding factor, a comparison of peripheral disease between the hybrid surgical group and primary stent extension group should be performed in future studies. Additionally, owing to the retrospective analysis of this small group, it is possible that some of the patients treated with the hybrid surgical approach might also have been 
good candidates for sole percutaneous treatment. To omit this bias, a prospective trial is warranted with randomization of patients into hybrid treatment or percutaneous stent extension.

In 1 of 14 patients, stent occlusion occurred without any additional interventional options. Although technically more challenging, the only possible interventional therapy in this context would be to create an AVF after thrombolysis or thrombectomy. As discussed with the patient, further therapy was waived because clinical symptoms did not interfere with daily activities.

A limitation of the extended endovascular technique is that it cannot be applied to all patients with postthrombotic obstructions peripheral to the femoral confluence. In cases with extensive involvement of the DFV and occlusion of the FV, adequate inflow in the CFV may be blocked, making it impossible to sustain stent patency. ${ }^{14}$ The DFV seems the best option for stent extension, as the FV shows less obstruction in most cases. All pathways contributing to stent inflow should be considered and analyzed preoperatively by duplex US and dedicated MR venography protocols. Specific attention should be paid to routes such as the axial extension, situated between the popliteal vein and contributing to inflow in the FVs. Based on the outcomes reported in this study, it is likely that inflow plays a superior role in stent patency compared with stent length. In centers without dedicated MR imaging protocols, intravascular US via the contralateral DFV can be performed. Consequently, the decision to perform a sole percutaneous procedure or a hybrid surgical procedure must be made perioperatively.

Future research should take all the aforementioned limitations into consideration. Moreover, only a small cohort sample with retrospective results is presented in this article. Ideally, randomized controlled trials should compare results between sole percutaneous treatment and surgical hybrid interventions. Finally, anticoagulation should be closely followed and documented by the treating physician to exclude it as a confounding factor in stent occlusion. In conclusion, stent placement through the femoral confluence into a dominant inflow vein seems to be a promising option in a carefully selected group of patients. 


\section{References}

1. Kahn SR, Shbaklo H, Lamping DL, et al. Determinants of health-related quality of life during the 2 years following deep vein thrombosis. J Thromb Haemost. 2008; 6:1105-12.

2. Enden T, Haig Y, Klow NE, et al. Long-term outcome after additional catheter-directed thrombolysis versus standard treatment for acute iliofemoral deep vein thrombosis (the CaVenT study): a randomised controlled trial. Lancet. 2012;379:31-8.

3. Mahnken AH, Thomson K, de Haan M, O'Sullivan GJ. CIRSE standards of practice guidelines on iliocaval stenting. Cardiovasc Intervent Radiol. 2014;37:889-97.

4. de Wolf MA, Jalaie $\mathrm{H}$, van Laanen $\mathrm{JH}$, et al. Endophlebectomy of the common femoral vein and arteriovenous fistula creation as adjuncts to venous stenting for post-thrombotic syndrome. Br J Surg. 2017;104:718-25.

5. Vogel D, Comerota AJ, Al-Jabouri M, Assi ZI. Common femoral endovenectomy with iliocaval endoluminal recanalization improves symptoms and quality of life in patients with posthrombotic iliofemoral obstruction. J Vasc Surg. 2012;55:129-35.

6. Puggioni A, Kistner RL, Eklof B, Lurie F. Surgical disobliteration of postthrombotic deep veinsendophlebectomy-is feasible. J Vasc Surg. 2004;39:1048-52.

7. Comerota AJ, Grewal NK, Thakur S, Assi Z. Endovenectomy of the common femoral vein and intraoperative iliac vein recanalization for chronic iliofemoral venous occlusion. J Vasc Surg. 2010; 52:243-7.

8. van Vuuren T, de Wolf MAF, Arnoldussen C, et al. Reconstruction of the femoro-ilio-caval outflow by percutaneous and hybrid interventions in symptomatic deep venous obstruction. Eur J Vasc Endovasc Surg. 2017;54:495-503.

9. Neglen P, Tackett TP Jr, Raju S. Venous stenting across the inguinal ligament. J Vasc Surg. 2008; 48:1255-61.

10. van Vuuren TM, Kurstjens RL, de Wolf MA, van Laanen $J H$, Wittens $C H$, de Graaf R. Stent extension into a single inflow vessel is a valuable option after endophlebectomy. Phlebology January 1, 2017. http://journals.sagepub.com/home/phla; published online. https://doi.org/10.1177/ 0268355517739766.

11. Behrendt CA, Heidemann F, Riess HC, et al. Open surgical treatment for postthrombotic syndrome. Phlebology. 2016;31(1 Suppl):48-55.

12. Kahn SR, Solymoss S, Lamping DL, Abenhaim L. Long-term outcomes after deep vein thrombosis: postphlebitic syndrome and quality of life. J Gen Intern Med. 2000;15:425-9.

13. Falcoz MT, Falvo N, Aho-Glele S, et al. Endovascular stent placement for chronic post-thrombotic symptomatic ilio-femoral venous obstructive lesions: a single-center study of safety, efficacy and quality-of-life improvement. Quant Imaging Med Surg. 2016;6:342-52.

14. Raju S, Ward M Jr, Davis M. Relative importance of iliac vein obstruction in patients with postthrombotic femoral vein occlusion. J Vasc Surg Venous Lymphat Disord. 2015;3:161-7. 



\section{Chapter 6}

Abdominal and pubic collateral veins as indicators of deep venous obstruction 


\section{Abstract}

\section{Objective}

Chronic deep venous obstruction can cause a significant loss of quality of life, although it can be treated successfully by stenting. A clear referral pattern for additional imaging is warranted in patients with lower limb complaints. The aim of this study was to determine the value of clinically visible abdominal wall collateral veins in the diagnosis of a potentially treatable deep venous obstruction.

\section{Methods}

A total of 295 patients referred for evaluation at a tertiary, venous clinic with a collateral vein on the abdominal wall or pubic bone, visible upon physical examination, were retrospectively analysed. They were compared with a randomly selected control group of 365 patients without such a collateral vein. Duplex ultrasound, magnetic resonance venography, computed tomography venography and conventional venography were used to determine the presence or absence of deep venous obstruction.

\section{Results}

Mean age of the group with a positive collateral was $43.5 \pm 13.7$ years $(6-76)$, compared to $44.7 \pm 14.2$ years $(16-89)$ in the control group. In the collateral group $66.1 \%$ were female compared to $63.3 \%$ in the control group. Sensitivity of the abdominal wall collateral vein for any obstruction at the level of the groin or more proximal was 53\% (95\% confidence interval $[\mathrm{Cl}]$, 48-57), specificity $86 \%(95 \% \mathrm{Cl}, 79-91)$, PPV 93\% (95\% Cl, 90-96) and NPV 32\% (95\% Cl, 28-37). Sensitivity was $68 \%(95 \% \mathrm{Cl}, 62-73)$ for higher degrees of post-thrombotic obstruction and $27 \%$ $(95 \% \mathrm{Cl}, 19-36)$ in iliac vein compression.

\section{Conclusions}

A collateral vein on the abdominal wall or across the pubic bone in patients with complaints of the lower limb has an excellent positive predictive value for deep venous obstructive disease at the level of the groin or higher. Such collateral veins should therefore not be removed and symptomatic patients could be offered further diagnostics and treatment. 


\section{Introduction}

Chronic deep venous obstruction can lead to various complaints. This is often accompanied by a significant reduction in quality of life similar to diabetes mellitus, congestive heart failure and chronic obstructive pulmonary disease. ${ }^{1,2}$ The main reason for developing such a chronic obstruction is inadequate recanalisation after deep vein thrombosis (DVT), resulting in the post-thrombotic syndrome (PTS). ${ }^{3}$ Annually, one to two per 1000 of the Western population will develop a DVT, ${ }^{4,5}$ $25-56 \%$ of whom develop PTS. ${ }^{2,6-9}$ In patients with a Lower Extremity Thrombosis classification 3 or 4, a DVT of the iliocaval or common femoral veins impairing the central venous outflow, the recanalisation rate is even worse and more patients suffer from PTS afterwards. ${ }^{10,11}$ The other main cause of chronic deep venous obstruction causing a central venous outflow obstruction is non-thrombotic iliac vein compression. ${ }^{3}$ This is often referred to as May-Thurner syndrome, which describes compression of the left common iliac vein (CIV) by the overlying right common iliac artery against the lumbar vertebral column. ${ }^{12}$ However, different anatomic compression variants exist. ${ }^{3,13}$ The prevalence of such an obstruction is unclear as these compressions are often not clinically relevant; e.g. compression of $>50 \%$ has been found in $24 \%$ of the general population. ${ }^{14}$

In cases of iliac and/or CFV obstruction where conservative therapy by compression stockings and mobilisation does not yield sufficient results, percutaneous transluminal angioplasty and stenting can be performed with good clinical success. ${ }^{15-18}$ However, adequate identification of possibly treatable pathology is necessary. Therefore, it is essential to establish a clear referral pattern. Whilst a history of deep vein thrombosis and venous claudication can be indicators of deep vein obstruction and mandate additional analysis, ${ }^{19,20}$ these signs do not indicate the level of obstruction and therefore possible treatability. Another parameter that might be useful in patient selection is the presence of a collateral vein visible on the abdominal wall or over the pubic bone during physical examination. ${ }^{21}$ This develops as an alternative outflow tract for central venous outflow obstruction and underlines the potential importance of collateral circulation in deep venous obstruction. ${ }^{22-24}$ Post-thrombotic obstruction or iliac vein compression can be diagnosed using duplex ultrasound (DUS), ${ }^{25,26}$ computed tomography venography (CTV), ${ }^{27}$ magnetic resonance venography $(\mathrm{MRV}),{ }^{26,27}$ intravascular ultrasound (IVUS), ${ }^{28}$ or venography. ${ }^{24,29}$ Since these techniques can be costly and sometimes invasive, it is vital to be as selective as possible when deciding to perform imaging studies to evaluate the deep veins.

The aim of this study was to determine the value of clinically visible abdominal wall collateral veins in the diagnosis of a potentially treatable deep venous obstruction and to investigate whether they should be used as a sign of referral for further diagnostics and potential treatment. 


\section{Methods}

\section{Study design and participants}

In this retrospective observational study we evaluated the clinical value of the presence of collateral veins on the abdominal wall or over the pubic bone (Figure 6.1), visible upon physical examination. This was assessed during first patient visit at our tertiary, outpatient clinic. Clinical value was based on the presence of deep venous obstruction on imaging in patients with and without these collaterals.

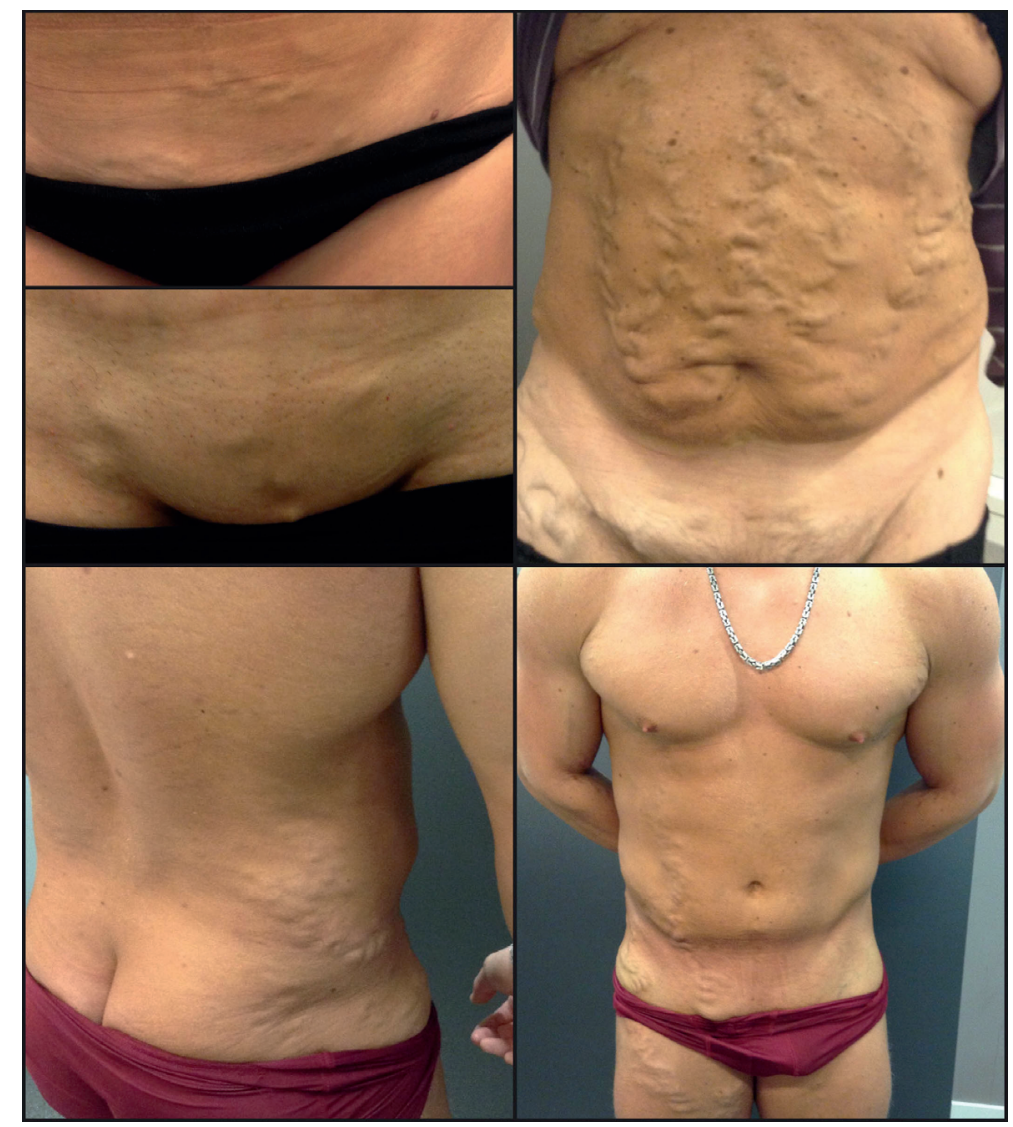

Figure 6.1 Examples of collateral veins over the pubic bone or abdominal wall.

Between May 2009 and November 2015, 1099 patients were referred to our tertiary, venous outpatient clinic. Patients who presented with a collateral vein on the abdominal wall or pubic bone (from hereon referred to as abdominal wall collateral), 
as determined by physical examination during their first visit, were included in this study. Patients also needed to have undergone DUS, CTV, MRV or conventional venography to diagnose presence of deep venous obstruction. Those who presented with an abdominal wall collateral during a later visit or who suffered a DVT $<1$ year before their visit were excluded. To create a control group, we randomly selected 403 patients from our database of patients referred to our outpatient clinic. These patients were generally referred because of suspicion of deep venous obstruction, based on complaints, a history of deep vein thrombosis or quickly recurrent varicosities. We first identified patients who did not present with an abdominal wall collateral. Thereafter, a researcher blinded for patient data randomly sampled 403 cases. Patients with insufficient imaging data of the deep venous system or a DVT <1 year before their visit were excluded from analysis in this control group. Information on complaints, venous claudication, history of DVT, previous venous interventions, C-class of the Clinical-Etiology-Anatomy-Pathophysiology (CEAP) classification for chronic venous disorders, Venous Clinical Severity Score (VCSS) and Villalta scale ${ }^{2}$ were collected for both groups of patients. Venous claudication was defined as the occurrence of heaviness or pain during walking, which subsides when the patient assumes a sitting or supine position with the leg elevated.

This study was approved by the Maastricht University Medical Centre institutional review board (METC 15-4-243). Individual patient consent was not obtained, as this is not required under Dutch law for retrospective studies.

\section{Procedures}

Imaging protocols described below were the same for both groups of patients.

\section{Duplex Ultrasound}

Patients underwent DUS during their first visit to our outpatient clinic. All DUS examinations were performed using a MyLab Alpha (Esaote, Genoa, Italy) or a ProSound Alpha 7 Premier machine (Hitachi Aloka, Tokyo, Japan). The deep venous system was visualised from the suprarenal inferior vena cava (IVC) to the CFV in the supine position, using a convex probe (frequency range 1-8 $\mathrm{MHz}$ ). Evaluation of the groin downwards was performed using a linear probe (frequency range 3-13 MHz) with the patient standing upright. However, for the purpose of this study only evaluation of the CFV was included from investigations in the erect position. Throughout the examination, available colour modalities were used to determine flow in both transverse and longitudinal planes. ${ }^{26}$ Flow division and intraluminal synechiae were considered as signs for post-thrombotic obstruction. Iliac vein compression was defined as $>50 \%$ lumen reduction compared with the diameter and transverse surface 
area of a normal contralateral common iliac vein or a healthy vessel segment more distal to the supposed compression.

\section{Magnetic Resonance Venography}

Patients referred to our dedicated venous clinic with a suspicion of outflow obstruction of the lower limb are routinely analysed with MRV. All MR examinations were performed on a 1.5-T magnetic resonance imaging system (Intera; Philips Healthcare, Best, the Netherlands). A dedicated 12-element phased-array peripheral vascular coil with a cranio-caudal coverage of $128 \mathrm{~cm}$ (Philips Healthcare, Best, the Netherlands) was used for signal reception. Patients were imaged in the supine position. Prior to contrast delivery, all patients underwent a standard twodimensional non-contrast enhanced balanced turbo field echo sequence to visualise the abdominal and pelvic veins. Then, a gadolinium-based contrast agent (Gadobutrol; Gadavist; Bayer Healthcare, Berlin, Germany) was administered intravenously at $1.0 \mathrm{ml} / \mathrm{s}(0.2 \mathrm{ml}$ per $\mathrm{kg}$ body weight, equals to $0.2 \mathrm{mmol} / \mathrm{kg})$ in the median cubital vein. Subsequently, a $20 \mathrm{ml}$ saline flush was injected at the same flow rate, using a remote controlled dual head injector (Spectris; Bayer Healthcare, Berlin, Germany). Acquisition of the first scan volume was started 30 seconds after contrast administration. A three-dimensional ultrafast gradient echo sequence (Ultrafast GE; THRIVE; Philips Healthcare, Best, the Netherlands) with fat suppression (spectral presaturation with inversion recovery) was used for high-resolution steady-state imaging of the venous vasculature, ensuring coverage of at least the popliteal veins up to the suprarenal IVC. ${ }^{26}$ Compression of a vein was defined as $>50 \%$ lumen reduction with the presence of collateral veins. Post-thrombotic obstruction was identified as the presence of intraluminal synechiae, which has been described and successfully performed before. ${ }^{26,30}$

\section{Computed Tomography Venography}

CTV was performed in patients who had a contraindication for MRV. Each examination was performed on a Siemens Flash CT scanner (Siemens Healthcare, Erlangen, Germany). An intravenous injection of $120 \mathrm{ml}$ iodine contrast medium (Ultravist 300; Bayer Healthcare, Berlin, Germany) was administered at $3.5 \mathrm{ml} / \mathrm{s}$ via an 18-gauge antecubital infusion catheter, followed by a saline flush of $40 \mathrm{ml}$ at $3.5 \mathrm{ml} / \mathrm{s}$. CT images were obtained and reconstructed with a slice thickness of $2.0 \mathrm{~mm}$ from the dome of the diaphragm to the toes, $180 \mathrm{~s}$ after the start of the injection. Images were routinely interpreted by a subspecialty trained cardiovascular radiologist on a regular picture archiving and communication system workstation using IMPAX 6.5 (Agfa Healthcare, Mortsel, Belgium). The primary criteria for deep venous obstruction were segmental non-visualisation, lack of opacification of the vein lumen, luminal 
narrowing with or without external compression, and identification of collateralisation. Compression was defined as $>50 \%$ lumen reduction compared to a normal vessel segment.

\section{Conventional venography}

Venography was performed in all cases in which treatment was indicated based on non-invasive imaging. In some cases it was performed when no pathology was found on DUS or MRV/CTV, yet deep venous obstruction was suspected on the basis of patient signs or symptoms. In the latter instance, anteroposterior venography was performed to identify typical signs of non-thrombotic iliac vein lesions, e.g. contrast translucency appearing as a filling defect; broadening/pancaking of the vein; and axial, transpelvic or ascending lumbar collaterals. ${ }^{31}$ Ultrasound guidance was used to puncture the femoral vein, circa $10 \mathrm{~cm}$ below the main deep femoral vein branch. This was done to identify any collateral veins above the level of the saphenofemoral junction, if present. Venography was performed during inspiration through a $6 \mathrm{~F}$ sheath (PreludePRO; Merit Medical Systems, South Jordan, UT, USA) with 10 cc of iodinated contrast (Ultravist 300; Bayer Healthcare, Berlin, Germany) at a flow velocity of $5 \mathrm{ml} / \mathrm{s}$. In the presence of aforementioned classic non-thrombotic iliac vein lesion signs, we determined a haemodynamically relevant downstream obstruction, i.e. significant iliac vein compression. The presence of collateral veins has been suggested to be of haemodynamic importance. ${ }^{32}$ No efforts were made to estimate iliac vein stenosis grade, since venography has never been validated to determine iliac vein lumen reduction accurately.

\section{Outcomes}

Primary outcome was the presence of deep venous obstruction of the CFV or more proximal. Obstruction was also divided into post-thrombotic disease and nonthrombotic iliac vein compression. Sub-analysis for patients with $>50 \%$ lumen reduction was performed in the post-thrombotic group. Obstruction was scored as positive if DUS or MRV/CTV identified the obstruction. Obstruction was scored as negative if both DUS and MRV/CTV did not show obstruction. If a segment was negative on one modality and not evaluated or not visualised on another modality it was also scored as negative. When no obstruction was identified on DUS, MRV or CTV, and conventional venography was performed, obstruction was scored as positive if one was identified on venography. 


\section{Statistical analysis}

Continuous data were presented as mean with standard deviation or median with interquartile range (IQR), depending on normality of distribution. An independent $t$-test or Man-Whitney $U$ test was performed to assess for differences between the two patient groups (presence and absence of abdominal wall collateral), depending on normality of distribution. Categorical data were presented using percentages and a Pearson's $\chi^{2}$ test was used to test for differences in patient characteristics. Fisher's exact test was used in cases where expected counts were less than five. $P$-values $\leq 0.05$ were considered as statistically significant. Sensitivity, specificity, positive predictive value (PPV) and negative predictive value (NPV) were calculated for the ability of abdominal wall collaterals to identify deep venous obstruction. Obstructions were stratified according to aetiology and a sub-analysis was performed for patients with $>50 \%$ lumen reduction, based on DUS. Diagnostic value analyses were performed using GraphPad Prism version 5.04 (GraphPad Software, San Diego, CA, USA), whereas all other analyses were performed using SPSS version 21.0 (IBM Corporation, Armonk, NY, USA).

\section{Results}

Of the 1099 patients who were referred to our clinic, 308 presented with an abdominal wall collateral. Eleven patients were excluded based on our exclusion criteria and an additional two patients were excluded because of insufficient imaging data. In the randomly selected control group of 403 patients, 30 patients had to be excluded according to our exclusion criteria and eight did not have full visualisation of the deep venous system. Thus, a total of 660 patients were analysed: 295 patients with an abdominal wall collateral and 365 patients without. (Figure 6.2)

Mean age of the group with a visible collateral was $43.5 \pm 13.7$ years $(6-76)$ compared to $44.7 \pm 14.2$ years $(16-89)$ in the control group $(P=0.276)$. Of all patients with a collateral, $66.1 \%$ were female compared to $63.3 \%$ in controls $(P=0.452)$. History of venous interventions was comparable between the two groups $(30.9 \%$ vs. $35.1 \%$, $P=0.549$ ). One patient in the control group had an abdominal wall collateral removed for cosmetic reasons in the past. Patients with a visible collateral more often had a history of DVT (79.3\% compared with $61.9 \%, P<0.001)$. Of those patients with a history of DVT or iatrogenic lesions, the group with a visible collateral presented at our outpatient clinic at 9.0 years (IQR, 3.0-18.0) after the event, compared to 6.0 years (IQR, 3.0-14.0) for controls $(P=0.061)$. Severity of disease, determined by venous claudication, $C$ of CEAP, VCSS and Villalta score, was not different between the two groups (Table 6.1). 


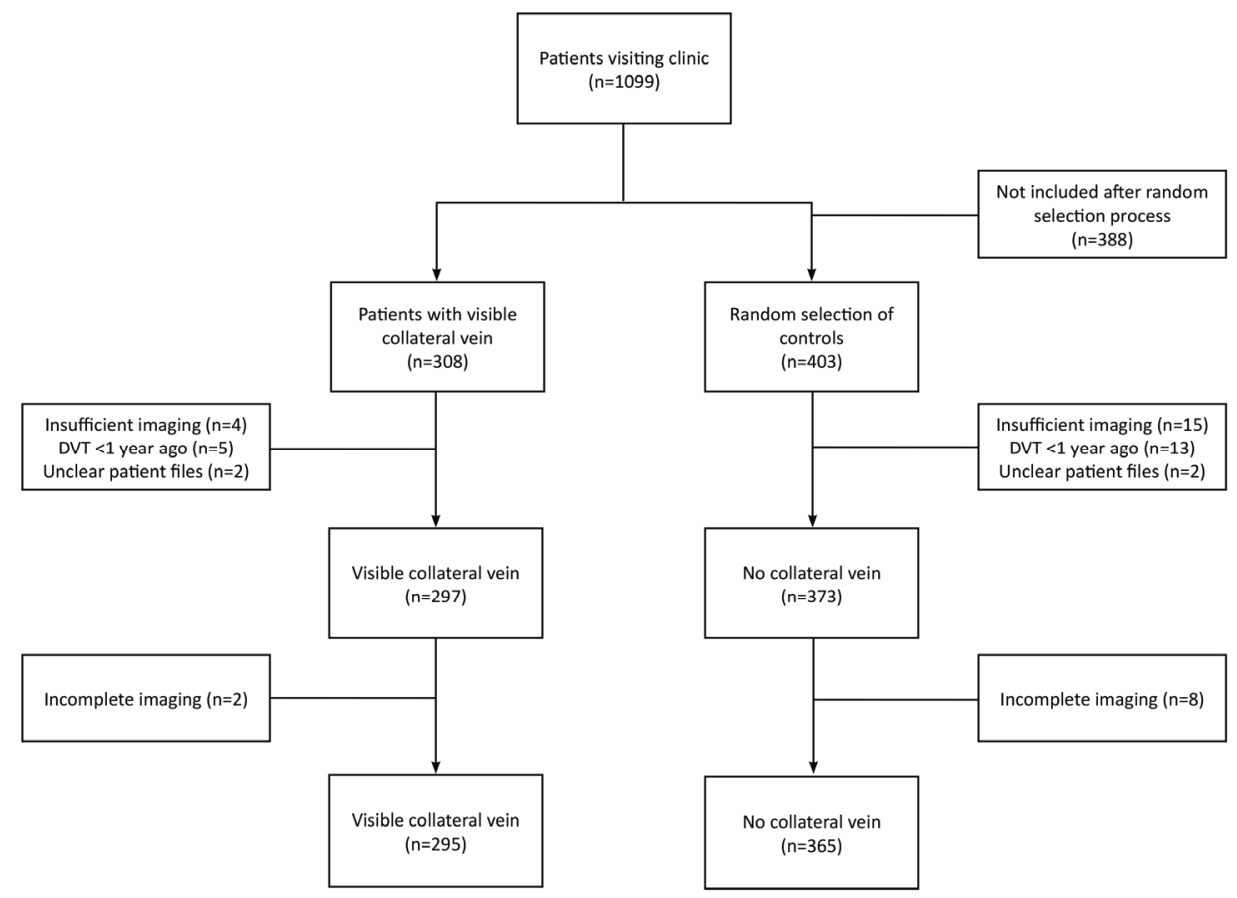

Figure 6.2 Flowchart of imaging studies. DVT, deep vein thrombosis.

A total of 522 obstructions on the level of the groin or higher were observed using DUS, CTV, MRV and venography; 404 (77.4\%) were of post-thrombotic nature, 116 (22.2\%) of non-thrombotic nature and in $2(0.4 \%)$ patients compression was observed without adequate visualisation of all lower limb vessel segments. Thus, the latter two patients might have post-thrombotic changes in the not visualised vessel segments, leading to exclusion of these patients for sub-analysis. Imaging with more than one modality was performed in 612 patients (92.7\%). More detailed information on identified pathology can be found in Figure 6.3. Of all detected post-thrombotic lesions, $60.4 \%$ were indentified in the collateral group and $39.6 \%$ in the control group $(P<0.001)$. Conversely, $26.7 \%$ of all detected non-thrombotic iliac vein compressions were identified in the collateral group compared with $73.3 \%$ in the control group $(P=0.015)$. 
Table 6.1 Patient characteristics.

\begin{tabular}{|c|c|c|c|}
\hline & $\begin{array}{l}\text { Abdominal wall } \\
\text { collateral }(n=295)\end{array}$ & $\begin{array}{l}\text { No abdominal wall } \\
\text { collateral }(n=365)\end{array}$ & $P$ \\
\hline Age - years (range) & $43.5 \pm 13.7(6-76)$ & $44.7 \pm 14.2(16-89)$ & 0.276 \\
\hline Female sex & $195(66.1)$ & $231(63.3)$ & 0.452 \\
\hline History of venous intervention ${ }^{*}$ & & & 0.549 \\
\hline No & $206(70.1)$ & 237 (64.9) & \\
\hline Superficial & 79 (26.9) & $113(30.9)$ & \\
\hline Deep & $13(4.5)$ & $20(5.4)$ & \\
\hline Removal of abdominal wall collateral & - & $1(0.3)$ & \\
\hline History of DVT & & & $<0.001^{\ddagger \ddagger}$ \\
\hline No & $55(18.6)$ & $137(37.5)$ & $<0.001^{\ddagger \ddagger}$ \\
\hline Yes & $234(79.3)$ & 226 (61.9) & $<0.001^{\text {拉 }}$ \\
\hline latrogenic lesion & $6(2.0)$ & $2(0.5)$ & 0.149 \\
\hline Years since event ${ }^{\dagger}$ & $9.0(I Q R, 3.0-18.0)$ & $6.0($ IQR, 3.0-14.0) & 0.061 \\
\hline Location of complaints $^{\ddagger}$ & & & 0.285 \\
\hline No complaints & $7(2.3)$ & $8(2.2)$ & \\
\hline Left leg & $163(55.4)$ & $163(45.2)$ & \\
\hline Right leg & $43(14.6)$ & $73(20.2)$ & \\
\hline Both legs & $76(25.9)$ & $109(30.2)$ & \\
\hline Abdomen/pelvis & $27(9.2)$ & $34(9.4)$ & \\
\hline Venous claudication $^{\S}$ & $156(54.2)$ & $172(49.0)$ & 0.194 \\
\hline VCSS & $7.4 \pm 3.5$ & $6.9 \pm 3.8$ & 0.137 \\
\hline Highest $C$ of CEAP ${ }^{* *}$ & & & 0.193 \\
\hline $\mathrm{C}_{0}$ & $21(7.2)$ & $39(10.8)$ & \\
\hline $\mathrm{C}_{1}$ & $24(8.2)$ & $29(8.0)$ & \\
\hline $\mathrm{C}_{2}$ & $56(19.2)$ & $51(14.1)$ & \\
\hline $\mathrm{C}_{3}$ & $96(33.0)$ & 121 (33.5) & \\
\hline $\mathrm{C}_{4}$ & $61(21.0)$ & 69 (19.1) & \\
\hline$C_{5}$ & $21(7.2)$ & $24(6.6)$ & \\
\hline $\mathrm{C}_{6}$ & $12(4.1)$ & $28(7.8)$ & \\
\hline Villalta scale $e^{t+}$ & & & 0.343 \\
\hline No PTS & $20(9.8)$ & $18(13.0)$ & \\
\hline Mild PTS & $74(36.3)$ & $46(33.3)$ & \\
\hline Moderate PTS & $69(33.8)$ & $38(27.5)$ & \\
\hline Severe PTS & $41(20.1)$ & $36(26.1)$ & \\
\hline
\end{tabular}

DVT: deep venous thrombosis, VCSS: venous clinical severity score, CEAP: Clinical-Etiology-AnatomyPathophysiology classification for chronic venous disease. Continuous variablesategorical variables are presented as number (\%). * 1 missing, $\$ 6$ missing, $¥ 5$ missing, $§ 21$ missing, ๆ 108 missing, ** 8 missing, †† Only given for patients with post-thrombotic disease $(n=401), 59$ missing, $¥ \ddagger$ Statistically significant.

The presence of an abdominal wall collateral visible during physical examination yielded a sensitivity of $53 \%(95 \% \mathrm{Cl}, 48-57)$, specificity of $86 \%(95 \% \mathrm{Cl}, 79-91)$, PPV of 93\% (95\% Cl, 90-96) and NPV of 32\% (95\% Cl, 28-37) for any obstruction found on imaging from the groin up to the right atrium (Table 6.2). Sub-analysis for postthrombotic obstruction demonstrated similar results with a sensitivity of $60 \%(95 \% \mathrm{Cl}$, $55-65)$, specificity of $86 \%(95 \% \mathrm{Cl}, 79-91)$, PPV of $92 \%(95 \% \mathrm{Cl}, 89-95)$ and NPV of $43 \%(95 \% \mathrm{Cl}, 37-49)$. Positive predictive value did not change when investigating 
degree of obstruction: $90 \%(95 \% \mathrm{Cl}, 85-94)$ for post-thrombotic obstructions with $>50 \%$ lumen reduction. Yet, the sensitivity increased $(68 \% ; 95 \% \mathrm{Cl}, 62-73)$, as did the NPV (58\%; 95\% Cl, 51-65). For the subgroup of non-thrombotic iliac vein compression PPV (61\%; 95\% Cl, 46-74) and sensitivity $(27 \% ; 95 \% \mathrm{Cl}, 19-36)$ were considerably lower (Table 6.2).

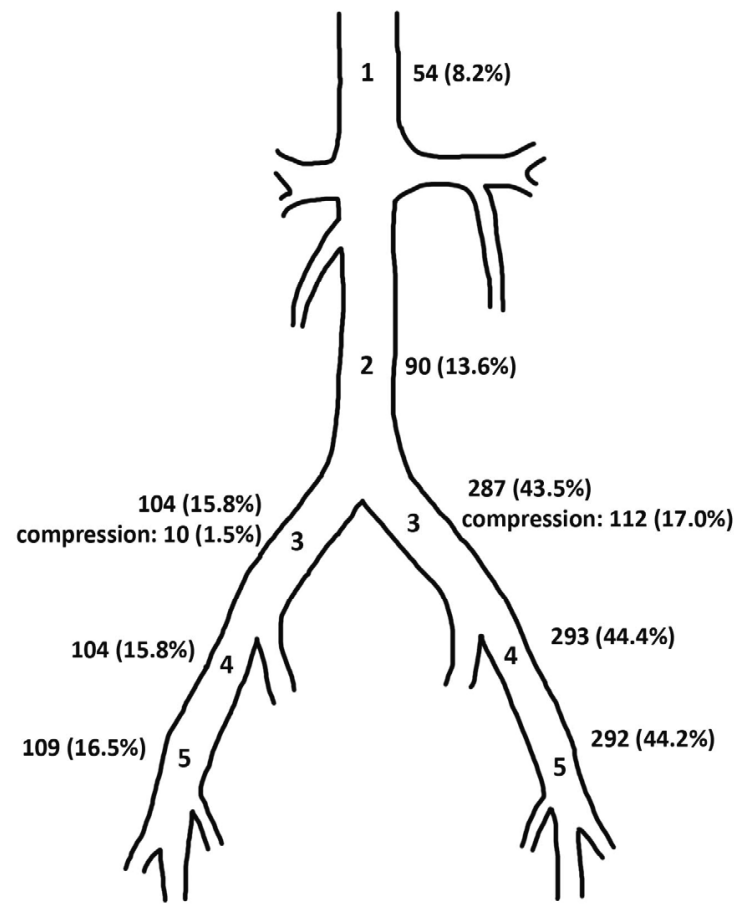

Figure 6.3 Pathology found with duplex ultrasound, magnetic resonance venography, computed tomography venography, and conventional venography $(n=660)$. lliac vein compression is only indicated in the absence of post-thrombotic changes of the iliac tract. 1, Suprarenal inferior vena cava; 2, infrarenal inferior vena cava; 3, common iliac vein; 4, external iliac vein; 5 , common femoral vein. In 138 cases (20.9\%) no obstruction was found. In 1 case $(0.2 \%)$ post-thrombotic occlusion of the right axillary and jugular veins was found.

Patients with a positive collateral vein sign without objectified obstruction often demonstrated some degree of venous pathology. For example, two patients demonstrated gonadal vein incompetence during venography. In two additional patients gonadal veins were dilated on MRV, and one patient showed slight compression of the left renal vein by the superior mesenteric artery on DUS, though not significant enough to warrant a Nutcracker syndrome diagnosis. Also, eight patients suffered from superficial vein incompetence in the area of the 
saphenofemoral junction, one from an aneurysmatic dilatation of the stump of a previously treated great saphenous vein, one of retrograde flow of the superficial epigastric vein and one from an incompetent internal pudendal vein. Four patients had no suggestion of venous pathology whatsoever.

Table 6.2 Diagnostic value of the abdominal wall collateral for different types of obstruction on the level of the groin or higher.

\begin{tabular}{lcccc}
\hline & Sensitivity & Specificity & $\begin{array}{c}\text { Positive predictive } \\
\text { value }\end{array}$ & $\begin{array}{c}\text { Negative predictive } \\
\text { value }\end{array}$ \\
\hline $\begin{array}{l}\text { Any obstruction } \\
(n=295,365)\end{array}$ & $53 \%$ & $86 \%$ & $93 \%$ & $32 \%$ \\
$\begin{array}{l}\text { Post-thrombotic } \\
\text { obstruction }\end{array}$ & $(95 \% \mathrm{Cl}, 48-57)$ & $(95 \% \mathrm{Cl}, 79-91)$ & $(95 \% \mathrm{Cl}, 90-96)$ & $(95 \% \mathrm{Cl}, 28-37)$ \\
$\begin{array}{l}(n=264,278) \\
\text { Post-thrombotic } \\
\text { obstruction }>50 \%\end{array}$ & $60 \%$ & $86 \%$ & $92 \%$ & $43 \%$ \\
$\begin{array}{l}(n=201,204) \\
\text { Non-thrombotic }\end{array}$ & $68 \%$ & $(95 \% \mathrm{Cl}, 79-91)$ & $(95 \% \mathrm{Cl}, 89-95)$ & $(95 \% \mathrm{Cl}, 37-49)$ \\
$\begin{array}{l}\text { obstruction } \\
(n=51,203)\end{array}$ & $27 \%$ & $86 \%$ & $90 \%$ & $58 \%$ \\
\hline
\end{tabular}

Number of patients included in analyses is first given for those with a collateral vein and then for those without.

\section{Discussion}

This study has shown that an abdominal wall collateral vein visible during physical examination has a positive predictive value of $93 \%$ for diagnosing deep venous obstructive disease at the level of the groin or higher. This clinical sign of venous obstruction has been described in literature before, ${ }^{21}$ as has its presence during duplex ultrasound examinations when it is often called a spontaneous Palma shunt. ${ }^{33}$ However, to our knowledge, no investigations were made with respect to its diagnostic abilities. The high PPV indicates that an abdominal wall collateral is a sign that patients should be referred for additional imaging. Specificity was fair (86\%), indicating few false negative test results, though negative predictive value was consistently very low (32\%), suggesting that no conclusions should be drawn in the absence of such a collateral vein during physical examination.

Post-thrombotic disease appears to have the most influence on the formation of a visible collateral vein, since sub-analysis yielded superior results to non-thrombotic iliac vein compression. This difference is substantiated by the fact that the collateral group more often had a history of DVT. Conversely, iliac vein compression without the presence of post-thrombotic abnormalities was more often present in patients without a visible abdominal wall collateral. The lower prevalence of abdominal wall 
collaterals in patients with non-thrombotic iliac vein compression therefore explains its inferior sensitivity in these patients.

An interesting observation is the removal of a collateral vein over the pubic bone for cosmetic reasons in one of the patients in the control group. This patient had lower limb complaints that remained stable after removal of the pubic collateral vein, which is certainly not always the case in our experience. We have also seen patients whose complaints deteriorated after removal of such a collateral vein. It is crucial that the physician realises that these collaterals are an essential part of the outflow of the leg and should never be removed, because the complaints related to the impaired outflow will likely increase. Visible collaterals on the abdominal wall or across the pubic bone are only the tip of the iceberg. In cases of CIV obstruction, blood flow can be diverted via the internal iliac vein through the pre-sacral and parametrial plexuses, ipsilateral ascending lumbar vein, ovarian veins, or paravertebral plexuses. ${ }^{22-24,26}$ When obstruction also involves the external iliac vein or CFV, the deep circumflex iliac vein, obturator vein or deep external pudendal vein can be activated too. ${ }^{22,23}$ Additionally, blood flow can be diverted through the superficial external pudendal, pubic and superficial epigastric veins, ${ }^{22,23}$ which leads to the visible collateral veins on the abdominal wall or across the pubic bone. Involvement of the IVC likely results in a more extensive collateral network, involving the paravertebral plexus and epigastric veins draining into the azygos system and the superior vena cava. ${ }^{23,26}$ These veins can lead to wide-ranging externally visible collateral formation.

Results could have been confounded by the presence of labial or scrotal varicosities. A distinction between such varicosities and collateral veins across the pubic bone could not always be determined due to succinct reporting. Isolated pudendal incompetence or incompetence of the saphenofemoral junction might have led to the presence of varicosities in the groin area. Nonetheless, better distinction between such varicosities and collateral veins would likely have led to an even higher PPV. Furthermore, save for four patients, all patients with a visible collateral showed some form of venous pathology. For example, incompetence in the area of the saphenofemoral junction might have led to retrograde flow in the superficial epigastric vein and therefore a visible collateral vein. Finally, it is conceivable that abdominal wall collaterals could also be connected to pelvic congestion syndrome, which is a complex phenomenon that is currently not completely understood and can be difficult to diagnose. ${ }^{34}$

Some limitations should be mentioned. Despite extensive imaging, the lack of intravascular ultrasound use might have influenced diagnosis of non-thrombotic iliac vein compression. Also, selection bias may be present as all patients were referred to our outpatient clinic with either complaints of the abdomen or lower limb, suspected to be of venous origin, or with known obstruction of the deep venous system. Collateral circulation can also be found in a number of different disorders. Ovarian tumours can cause dilated ovarian veins and gestational trophoblastic neoplasms can 
lead to a more pronounced uterine plexus, as can uterine arteriovenous malformations. ${ }^{23}$ These deep collateral networks could theoretically lead to a collateral sign visible upon physical examination. Finally, portal hypertension can cause collateral formation and is known to demonstrate clinically visible abdominal veins. $^{35}$ Studies in a general population with complaints of the lower limb could determine whether such a bias was actually present.

\section{Conclusion}

A collateral vein on the abdominal wall or across the pubic bone in patients with complaints of the lower limb has an excellent positive predictive value for potentially treatable deep venous obstructive disease at the level of the groin or higher. This warrants closer inspection of the groin and abdominal area in all patients with lower limb symptoms. Such collateral veins should never be removed for aesthetic purposes and symptomatic patients should be referred for additional imaging, and possibly treatment, of their obstruction. 


\section{References}

1. Carradice D, Mazari FA, Samuel N, Allgar V, Hatfield J, Chetter IC. Modelling the effect of venous disease on quality of life. Br J Surg. 2011;98(8):1089-98.

2. Kahn SR, Shbaklo H, Lamping DL, et al. Determinants of health-related quality of life during the 2 years following deep vein thrombosis. J Thromb Haemost. 2008;6(7):1105-12.

3. Meissner MH, Eklof B, Smith PC, et al. Secondary chronic venous disorders. J Vasc Surg. 2007;46 Suppl S:68S-83S.

4. White RH. The epidemiology of venous thromboembolism. Circulation. 2003;107(23 Suppl 1):14-8.

5. Naess IA, Christiansen SC, Romundstad P, Cannegieter SC, Rosendaal FR, Hammerstrom J. Incidence and mortality of venous thrombosis: a population-based study. J Thromb Haemost. 2007;5(4):692-9.

6. Mohr DN, Silverstein MD, Heit JA, Petterson TM, O'Fallon WM, Melton LJ. The venous stasis syndrome after deep venous thrombosis or pulmonary embolism: a population-based study. Mayo Clin Proc. 2000;75(12):1249-56.

7. Prandoni P, Villalta S, Bagatella $\mathrm{P}$, et al. The clinical course of deep-vein thrombosis. Prospective longterm follow-up of 528 symptomatic patients. Haematologica. 1997;82(4):423-8.

8. Schulman $\mathrm{S}$, Lindmarker $\mathrm{P}$, Holmstrom $\mathrm{M}$, et al. Post-thrombotic syndrome, recurrence, and death 10 years after the first episode of venous thromboembolism treated with warfarin for 6 weeks or 6 months. J Thromb Haemost. 2006;4(4):734-42.

9. Tick LW, Kramer MH, Rosendaal FR, Faber WR, Doggen CJ. Risk factors for post-thrombotic syndrome in patients with a first deep venous thrombosis. J Thromb Haemost. 2008;6(12):2075-81.

10. Strijkers RH, Arnoldussen CW, Wittens CH. Validation of the LET classification. Phlebology. 2015;30(1 Suppl):14-9.

11. Akesson H, Brudin L, Dahlstrom JA, Eklof B, Ohlin P, Plate G. Venous function assessed during a 5 year period after acute ilio-femoral venous thrombosis treated with anticoagulation. Eur J Vasc Surg. 1990;4(1):43-8.

12. May R, Thurner J. [A vascular spur in the vena iliaca communis sinistra as a cause of predominantly left-sided thrombosis of the pelvic veins]. Z Kreislaufforsch. 1956;45(23-24):912-22.

13. Neglen P, Berry MA, Raju S. Endovascular surgery in the treatment of chronic primary and postthrombotic iliac vein obstruction. Eur J Vasc Endovasc Surg. 2000;20(6):560-71.

14. Kibbe MR, Ujiki M, Goodwin AL, Eskandari M, Yao J, Matsumura J. Iliac vein compression in an asymptomatic patient population. J Vasc Surg. 2004;39(5):937-43.

15. Neglen P, Hollis KC, Olivier J, Raju S. Stenting of the venous outflow in chronic venous disease: longterm stent-related outcome, clinical, and hemodynamic result. J Vasc Surg. 2007;46(5):979-90.

16. Wen-da W, Yu Z, Yue-Xin C. Stenting for chronic obstructive venous disease: A current comprehensive meta-analysis and systematic review. Phlebology. 2016;31(6):376-89.

17. Seager MJ, Busuttil A, Dharmarajah B, Davies AH. Editor's Choice - A Systematic Review of Endovenous Stenting in Chronic Venous Disease Secondary to Iliac Vein Obstruction. Eur J Vasc Endovasc Surg. 2016;51(1):100-20.

18. de Wolf MA, de Graaf R, Kurstjens RL, Penninx S, Jalaie H, Wittens CH. Short-Term Clinical Experience with a Dedicated Venous Nitinol Stent: Initial Results with the Sinus-Venous Stent. Eur J Vasc Endovasc Surg. 2015;50(4):518-26.

19. Wittens C, Davies AH, Baekgaard N, et al. Editor's Choice - Management of Chronic Venous Disease: Clinical Practice Guidelines of the European Society for Vascular Surgery (ESVS). Eur J Vasc EndovasC Surg. 2015;49(6):678-737.

20. Kurstjens $R$, de Wolf $M$, de Graaf $R$, Wittens $C$. Hemodynamic changes in iliofemoral disease. Phlebology. 2014;29(1 suppl):90-6.

21. Wittens $\mathrm{CH}$, Bukkems SF, Toonder IT. Abdominal wall venous collaterals: the latent clinical sign for central chronic venous obstruction. Circulation. 2010;122(20):2089-90.

22. Thomas ML, Fletcher EW, Cockett FB, Negus D. Venous collaterals in external and common iliac vein obstruction. Clin Radiol. 1967;18(4):403-11. 
23. Umeoka S, Koyama T, Togashi K, Kobayashi H, Akuta K. Vascular dilatation in the pelvis: identification with CT and MR imaging. Radiographics. 2004;24(1):193-208.

24. Neglen P, Raju S. Balloon dilation and stenting of chronic iliac vein obstruction: technical aspects and early clinical outcome. J Endovasc Ther. 2000;7(2):79-91.

25. Labropoulos N, Borge M, Pierce K, Pappas PJ. Criteria for defining significant central vein stenosis with duplex ultrasound. J Vasc Surg. 2007;46(1):101-7.

26. Arnoldussen $\mathrm{CW}$, Toonder I, Wittens $\mathrm{CH}$. A novel scoring system for lower-extremity venous pathology analysed using magnetic resonance venography and duplex ultrasound. Phlebology. 2012;27 Suppl 1:163-70.

27. Arnoldussen $\mathrm{CW}$, de Graaf $\mathrm{R}$, Wittens $\mathrm{CH}$, de Haan MW. Value of magnetic resonance venography and computed tomographic venography in lower extremity chronic venous disease. Phlebology. 2013;28 Suppl 1:169-75.

28. Neglen P, Raju S. Intravascular ultrasound scan evaluation of the obstructed vein. J Vasc Surg. 2002;35(4):694-700.

29. Nicolaides AN. Investigation of chronic venous insufficiency: A consensus statement (France, March 59, 1997). Circulation. 2000;102(20):E126-63.

30. Fraser DG, Moody AR, Morgan PS, Martel A. Iliac compression syndrome and recanalization of femoropopliteal and iliac venous thrombosis: a prospective study with magnetic resonance venography. J Vasc Surg. 2004;40(4):612-9.

31. Raju S, Neglen P. High prevalence of nonthrombotic iliac vein lesions in chronic venous disease: a permissive role in pathogenicity. J Vasc Surg. 2006;44(1):136-43; discussion 44.

32. Kurstjens RL, de Wolf MA, van Laanen JH, de Haan MW, Wittens $\mathrm{CH}$, de Graaf R. Hemodynamic significance of collateral blood flow in chronic venous obstruction. Phlebology. 2015;30(1 Suppl):2734.

33. Mendoza E, Lattimer CR. Examination of superficial veins in the presence of deep venous disease. In: Mendoza E, Lattimer CR, Morrison N, eds. Duplex ultrasound of superficial leg veins: Springer; 2014: 279-83.

34. Tu FF, Hahn D, Steege JF. Pelvic congestion syndrome-associated pelvic pain: a systematic review of diagnosis and management. Obstet Gynecol Surv. 2010;65(5):332-40.

35. Pillai AK, Andring B, Patel A, Trimmer C, Kalva SP. Portal hypertension: a review of portosystemic collateral pathways and endovascular interventions. Clin Radiol. 2015;70(10):1047-59. 


\section{Chapter 7}

Venous stent patency may be affected by collateral vein lumen size 


\section{Abstract}

\section{Purpose}

Deep venous thrombosis causes blood flow deviation. It is hypothesized that with stent placement, developed collateral veins become redundant. This article evaluates the relation between the surface area of the collaterals and stent patency.

\section{Methods}

The azygos and hemiazygos veins were identified and the largest surface area was measured at thoracic level. Patency rates of stented tracts were evaluated and related to collateral vein lumen size.

\section{Results}

The vena cava occlusion and the azygos and hemiazygos vein surface area measurements were positive and statistically significant related (OR 1.01, 95\% $\mathrm{Cl} 1.003-1.019, P=0.004$ ) respectively (and OR 1.007, 95\% Cl 1.001-1.013, $P=0.004$ ). An azygos surface area measurement of 23 $(P<0.001)$ and hemiazygos surface area measurement of $40(P=0.008)$ was shown as cut-off point related to higher occlusion rates.

\section{Conclusions}

The surface area of major venous collateral pathways seems to be related to stent occlusion in deep venous interventions. 


\section{Introduction}

Deep venous thrombosis (DVT) is quite common and causes blood flow deviation in the venous system. ${ }^{1,2}$ Pathophysiological changes after a DVT result in alternative drainage depending on the degree of recanalization. This venous recanalization, with vein wall fibrosis and synechiae formation, is a complex process in which many factors seem to play a role. When normal anatomical routes become chronically affected due to these post-thrombotic synechiae and vein wall fibrosis, ${ }^{3-6}$ collateral veins are burdened with increased blood flow. Specifically, in caval vein obstruction, the azygos and hemiazygos, gonadal, spinal or abdominal veins have been described as predominant collaterals. ${ }^{7,8}$ Normally, the azygos and hemiazygos veins are responsible for draining the thoracic wall and upper lumbar region via posterior intercostal veins. After post-thrombotic changes of the inferior caval vein, the azygos and hemiazygos veins become more important for blood drainage. ${ }^{8}$ The recognition of these collateral pathways as well as the extent of acute and chronic obstructions can be evaluated by means of magnetic resonance venography (MRV), computed tomography, duplex ultrasound (DUS) and phlebography. ${ }^{9-11}$

More extensive DVT, involving not only the inferior caval vein but also the iliac (cavoiliac) tract, can obstruct the outflow of the whole leg. ${ }^{12}$ Subsequently, patients are more prone to develop complaints like edema and pain related to the postthrombotic syndrome (PTS). ${ }^{13,14}$ Severe cases of PTS are less responsive to conservative treatment and require more extensive percutaneous venous recanalization with trans-luminal angioplasty (PTA) and stent placement. ${ }^{15}$ Studies have shown favorable clinical outcomes ${ }^{16}$ with occlusions of the inferior caval and iliac veins to be the most challenging cases to treat. Secondary patency rates up to $93 \%$ have been described, whereas primary patency rates were $53 \% .{ }^{17}$

It is hypothesized that with PTA and stent placement, developed collateral veins become redundant when the normal physiological route is restored. For example, a restored inferior caval vein will inevitably mean that enlarged azygos and hemiazygos collateral veins would become less important. However, the hemodynamic importance of collateral pathways and their effects on stent patency after an intervention has not yet been investigated. In an effort to explore various explanations for stent failure, the collateral veins of treated patients were reanalyzed on preoperative MRV scans.

Specifically, the aim of this article was to evaluate the relation between the collateral vein lumen size of the azygos and hemiazygos collaterals and stent patency. 


\section{Methods}

This research was approved by the Ethical Committee of Maastricht (METC 16-4-183). All eligible patients treated for post-thrombotic (cavo) iliofemoral deep venous obstructions between 2012 and 2015 were evaluated. All patients experienced venous complaints and/or signs interfering with daily activities. Venous complaints and signs were defined as pain interfering with daily activities, and/or clinical signs classified according to the CEAP classification. Additionally, venous claudication was scored and regarded as a clinical complaint to perform deep venous treatment. Subjects treated for an acute thrombosis were excluded as natural recanalization did not occur and it was assumed that collateral veins would not have had ample opportunity to develop $(n=8)$. When the azygos or hemiazygos veins could not be identified with certainty, the subject was excluded $(n=1)$. Also, subjects treated with novel anticoagulant therapy were excluded as its effect on stent patency has not yet been investigated into detail ( $n=15$ of which eight subjects had an occlusion somewhere in the follow up period). With these exclusion criteria, 199 patients were eligible for analysis.

Baseline characteristics like age, gender, number of DVTs and extensiveness of postthrombotic changes were analyzed.

\section{Magnetic resonance venography}

A full description of the MR protocol has been described previously. ${ }^{18,19}$ In short, a 1.5-T MRI system (Intera, Philips Healthcare, Best, The Netherlands) was used. The venous vasculature was imaged by a threedimensional threedimensional ultrafast gradient echo sequence (Ultrafast GE, THRIVE, Philips Healthcare) with fat suppression (spectral presaturation with inversion recovery, SPIR). Gadolinium based contrast (Gadobutrol; Gadavist, Bayer HealthCare, Berlin, Germany) was intravenously administered before scanning the subjects. A full lower extremity and abdominal scan was performed to ensure coverage of the lower veins up to the upper part of the inferior caval vein. The imaging evaluation was focused on the identification of chronic venous obstruction and related collateral patterns. Intraluminal synechiae with formation of collateral veins were considered as signs for post-thrombotic obstruction.

Based on MRV findings, the lower extremity postthrombotic changes were subdivided into two scales. The first scale indicated obstruction of the common femoral and iliac veins. Subsequently, the involvement of the inferior vena cava resulted in the second scale.

The azygos and hemiazygos veins were identified and the largest found collateral vein lumen size was measured at a thoracic level (Figure 7.1). This collateral vein lumen size measurement was referred to as Region of Interest (ROI) and expressed as $\mathrm{mm}^{2}$. 


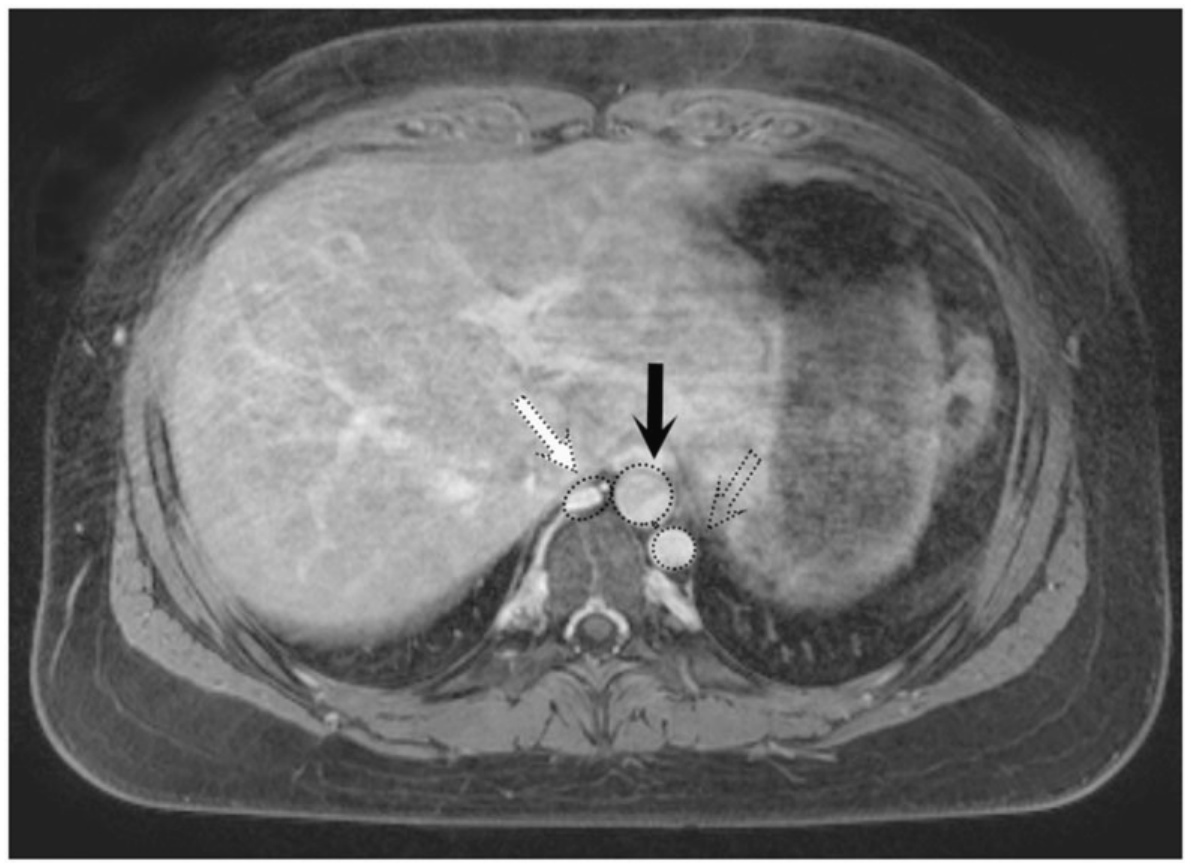

Figure 7.1 Example of ROI measurement at thoracic level. ROI: Region of Interest; White arrow: azygos vein, black arrow aorta, grey open arrow hemiazygos vein.

\section{Treatment and follow up}

A thorough description of the interventions has been described before. ${ }^{18}$

Iliac stents were positioned at least at the level of the common iliac vein (CIV) confluence, generally extending the spinous process. The iliac part was treated with a 18-14 $\mathrm{mm}$ diameter stent depending on the vein lumen after ballooning. The femoral vein was stented with $14-10 \mathrm{~mm}$ stents. For the caval obstructions, a sinus XL $24 \mathrm{x}$ 80-160 mm (Optimed, Ettlingen, Germany) was used as these stents were available in larger diameters. Bilateral treated subjects were stented in the caval vein. The iliac confluence was treated by either self-expandable stents inside the inferior vena cava (IVC) stent or by high radial force balloon-expandable stents at the same level. In both cases, bilateral iliac extensions were performed using nitinol stents. This second technique was developed to minimize stent related compression issues causing stent stenosis or occlusion.

Postintervention, all subjects were treated with anticoagulant therapy. Patients used Coumadin therapy with a target INR level between 3 and 4 for a minimum of 6 months. 
During routine clinical follow up, complaints were evaluated and entered into a digital database. A DUS was performed to analyze the stent patency and possible stent related complications. This DUS evaluation was performed before discharge, at 2 weeks, 6 weeks, 3 months, 6 months, 12 months postintervention and annually thereafter.

Primary patency was scored as open stents with adequate venous flow and flow out of stenosis requiring any additional intervention. All subjects with an occlusion during the follow up period, were included in the secondary patency group. A thrombolysis with additional stenting and an arterio-venous fistula was performed when the patient experienced recurrent or new venous complaints. In patients without clinical complaints despite stent obstruction or an occlusion existing more than 3 weeks, this therapy was waived.

\section{Statistics}

The Shapiro-Wilk test was used to test the normality of the distribution. Continuous, normally distributed data were presented as a mean with their standard deviation. Non-normally distributed interval data were presented with lowest and highest scores. Categorical data were presented as frequencies and percentages.

The Spearman correlation coefficient $(r)$ was used to inspect relationships between continuous variables. Logistic regression univariate analysis was used to measure the odds ratio (OR) between the measured collateral vein lumen size and the occlusion of stents. To show the distribution of collateral vein lumen size measurements and occlusion of stents, a ROC curve was performed. The distribution of vein collateral vein lumen size measurements was categorized and differences were compared using a chi-square test.

An $\alpha$ of $<0.05$ was considered to be statistically significant. Data were analyzed using IBM SPSS statistics v23.0 (IBM Corporation, Armonk, NY, USA)

\section{Results}

Table 7.1 shows the demographics of all included patients. The mean age was 43.2 years ( \pm 12.7 , range $17-75$ ) and $67 \%$ of the patients were female. About $27 \%$ of the patients had more than one deep vein thrombosis in the treated leg. With a median follow up of 14 months (8-27), the primary patency of this cohort was $80 \%$. An occlusion occurred in $20 \%$ somewhere during the follow up period. After a median follow up of 14 months, a total of $10 \%$ had an occluded stent.

A median of two stents were used to treat the postthrombotic lesions. In 138 (70\%) patients, this was performed using a Sinus Venous stent. In 23 (12\%) patients, an SXL was used for the caval obstruction and Andra or Obliquus stent for the iliac 
confluence. Furthermore, SXL stents ( $n=31,15.5 \%)$, Veniti stents $(n=3,1 \%)$, Zilver vena $(n=3,1 \%)$ or Venovo $(n=1,0.5 \%)$ stents were used.

Table 7.1 Demographics.

\begin{tabular}{llcc}
\hline Age, yr mean \pm SD, range & & $43.2 \pm 12,7$ & $(17-75)$ \\
Gender N, \% & Male & 66 & $33 \%$ \\
& Female & 133 & $67 \%$ \\
BMI (median, IQR) & & 27.1 & $(23.5-30.2)$ \\
Coagulopathy & & $44 / 148$ & $30 \%$ \\
PTS scale N, \% & Scale 1 & 176 & $88 \%$ \\
& Scale 2 & 23 & $12 \%$ \\
Number DVT's & 1 & 145 & $73 \%$ \\
& $>1$ & 54 & $27 \%$ \\
Time from DVT, mnth median, IQR & & 51 & $(22-134)$ \\
\hline
\end{tabular}

PTS: post-thrombotic syndrome; Yr: year; N: number; DVT: deep venous thrombosis; mnth: month.

\section{Collateral vein lumen size measurements and post-thrombotic involvement of the inferior caval vein}

The median score for azygos collateral vein lumen size was $20.5 \mathrm{~mm}^{2}$ (diameter $6.5 \mathrm{~mm}, I Q R$ 14-32) and the median score for the hemiazygos collateral vein lumen size was $33.5 \mathrm{~mm}^{2}$ (diameter $10.5 \mathrm{~mm}$, IQR 21-51).

Twenty-three patients (12\%) had post-thrombotic fibrosis in the inferior vena cava on the preinterventional MR scan. The univariate relationship between the vena cava occlusion and the collateral vein lumen size measurements was positive and statistically significant for the azygos vein (OR 1.01, 95\% Cl 1.003-1.019, $P=0.004$ ). Also, hemiazygos collateral vein lumen size measurements were statistically significant higher in patients with involvement of the vena cava compared to patients without the involvement of the vena cava (and OR 1.007, 95\% Cl 1.001-1.013, $P=0.004)$.

\section{Time to latest DVT and collateral vein lumen size}

As collateralization takes time and recanalization can occur up to 12 months after a DVT, ${ }^{20}$ the time between the latest known DVT event and MR scan was evaluated. The median time between the latest DVT and the MRV scan was 51 months (IQR 22-134). To analyze the relation between the timespan and the collateral vein lumen size, a correlation analysis was performed. A Spearman correlation test did not show a significant correlation for both the ayzgos or the hemiazygos vein ( $r-0.010, p$ 0.89; r-0.036, p 0.61, respectively). 
Also, the number of DVTs before stenting and the relation to enlarged collateral vein lumen size measurements was analysed. This analysis did not show a statistical significant difference for the azygos or hemiazygos vein $(P=0.48, P=0.67$, respectively).

\section{Collateral vein lumen size measurements and occlusion of stents}

The collateral vein lumen size measurements were subdivided into categories to analyze its relation to stent occlusion. Table 7.2 shows the collateral vein lumen size measurements of the azygos vein related to stent patency. A collateral vein lumen size measurement of $23 \mathrm{~mm}^{2}$ (diameter $>7.3 \mathrm{~mm}$ ) was shown as the cut-off point related to higher occlusion rates $(P<0.001)$.

Table 7.2 Azygos ROI and diameter measurements related to stent patency.

\begin{tabular}{lcc}
\hline ROI (diam) azygos & Patent $(\mathrm{N}, \%)$ & Occluded $(\mathrm{N}, \%)$ \\
\hline$<15(<4.8)$ & $51(94)$ & $3(6)$ \\
$15-20(4.8-6.4)$ & $43(94)$ & $3(6)$ \\
$21-32(6.5-10.2)$ & $40(74)$ & $14(26)$ \\
$>32(>10.2)$ & $24(53)$ & $21(47)$ \\
\hline
\end{tabular}

Table 7.3 shows the collateral vein lumen size measurements of the hemiazygos vein related to stent patency. A collateral vein lumen size measurement of 40 (diameter $>12.7)$ was shown as the cut-off point related to higher occlusion rates $(P=0.008)$.

Table 7.3 Hemiazygos ROI and diameter measurements related to stent patency.

\begin{tabular}{lcc}
\hline ROI (diam) hemiazygos & Patent $(\mathrm{N}, \%)$ & Occluded $(\mathrm{N}, \%)$ \\
\hline$<21(<6.7)$ & $47(87)$ & $7(13)$ \\
$21-33(6.7-10.5)$ & $41(89)$ & $5(11)$ \\
$34-50(10.6-15.9)$ & $40(77)$ & $12(23)$ \\
$>50(>15.9)$ & $30(64)$ & $17(36)$ \\
\hline
\end{tabular}

A ROC analysiswas performed to discriminate between the occurrence of stent occlusion. For the azygos vein, an area under the curve (AUC) of $77 \%(95 \% \mathrm{Cl}$ $0.69-0.84$ ) (Figure 7.2 ) and for the hemiazygos vein, an AUC of $67 \%$ (95\% Cl 0.57-0.77) were found (Figure 7.3).

Univariate analysis of variance on occlusion rate and azygos collateral vein lumen size showed a significant effect when controlled for BMI, age, gender and coagulopathy $(P<0.001)$. When controlled for hemiazygos collateral vein lumen size, the effect of azygos size still remained. No significant effect of hemiazygos collateral vein lumen size and stent occlusion was found when controlled for BMI, age, gender risk of thrombosis $(P=0.78)$. 


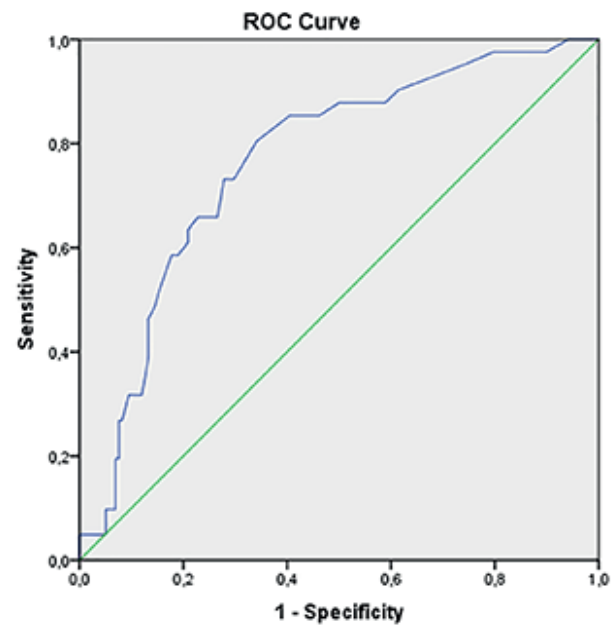

Figure 7.2 A ROC analysis for occurrence of stent occlusion and ROI of the azygos vein. An area under the curve (AUC) of $77 \%(95 \% \mathrm{Cl} 0.69-0.84)$, with increasing azygos vein ROI, a higher number of stent occlusions is seen. ROI: Region of Interest.

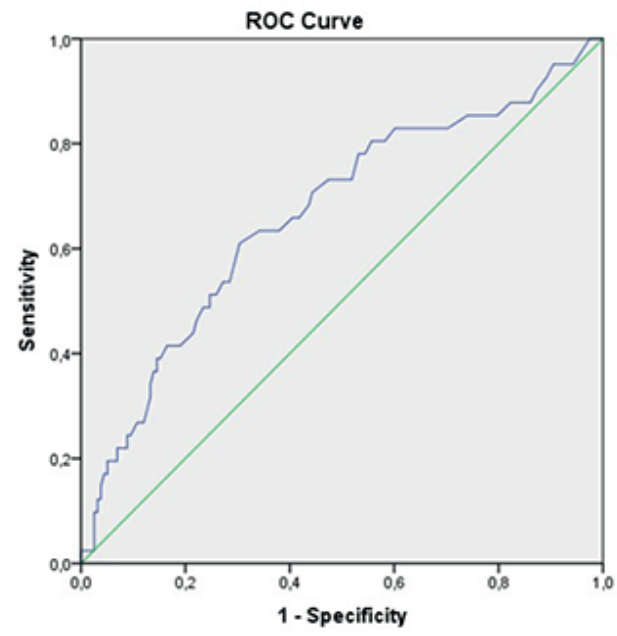

Figure 7.3 A ROC analysis for occurrence of stent occlusion and ROI of the hemiazygos vein. An area under the curve (AUC) of $67 \%(95 \% \mathrm{Cl}$ 0.57-0.77) with increasing hemiazygos vein ROI, a higher number of stent occlusions is seen. ROI: Region of Interest.

\section{Type of obstruction and occlusion of stents}

In order to analyze whether there was a relation between the extent of the postthrombotic changes and the risk of stent occlusion, a Pearson correlation test was 
performed. In $88 \%$ of the patients, a scale 1 was found indicating post-thrombotic changes up to and thus excluding the vena cava. The risk of stent occlusion was not statistically significant related to the extent of post-thrombotic changes $(P=0.89)$. Furthermore, when the number of DVTs was dichotomized into one or multiple events, this was not significantly related to the occurrence of stent occlusion $(P=0.41)$.

\section{Discussion}

Recanalization after a DVT results in the development of intraluminal synechiae or fibrotic vessels. ${ }^{6}$ Therefore, the veins are incapable of normal expansion and thus limited in transporting blood especially when volumes increase during exercise. Although collateral veins function as detours, they seem to be an inferior conduit and are less able to adopt the function of non-diseased veins. ${ }^{21}$ Subsequently, the main goal of venous stenting is to restore the original pre-thrombotic venous anatomy as to transport blood more effectively and reduce clinical complaints. It is expected that the main volume of flow should pass through the stented segments. However, competitive outflow routes might diverge the blood away from the stents and as a consequence increase the risk of thrombus formation and thrombotic stent occlusion. This fact has been described by Neglen and Raju explaining that the presence of collaterals could be an indicator of more severe stenosis, but significant stenosis can also be apparent without collateral formation. ${ }^{22}$ Since thrombotic stent occlusion may result in the recurrence or worsening of clinical complaints, the aim was to search for a predictor of stent occlusion in order to provide more realistic expectations for physicians and patients. As a consequence, the relation between stent patency and the collateral vein lumen size of the azygos and hemiazygos veins were evaluated. The results presented, show that patients with a larger collateral vein lumen size were more prone to stent occlusion.

According to the literature, the azygos and hemiazygos veins are responsible for venous blood flow towards the heart in patients with a caval obstruction. ${ }^{7,8}$ Accordingly, the results in this article show the importance of the azygos and hemiazygos veins when properly quantified according to their lumen size. This means that physicians could focus on the analysis of particular collateral pathways before contemplating interventional treatment. As a consequence, the chance of stent occlusion might be predicted and provide a more realistic expectation for patients. The probability of stent occlusion based on the azygos and hemiazygos collateral vein sizes is shown in the ROC curves with an AUC of $74 \%$ and $67 \%$, respectively. To make a precise discrimination, a high sensitivity and specificity would be ideal. Consequently, a cut off value with a high sensitivity should be determined to select those patients at risk for a stent occlusion. With the results of this study, a clear cut-off factor could not 
be pointed out, as there are subjects with high ROIs without occlusion and subjects with low ROls with thrombotic stent occlusions. However, a significant increase in stent occlusion was seen in azygos collateral vein lumen size measurements above $23 \mathrm{~mm}^{2}$ (diameter $>7.3 \mathrm{~mm}$ ) and hemiazygos measurements above $40 \mathrm{~mm}^{2}$ (diameter>12.7).

As stent failure after deep venous reconstruction seems to be a multifactorial process, some other factors were analyzed to exclude them as contributing factors in thrombotic stent occlusion. To examine whether the recovery time after a DVT had a significant effect on the collateral vein lumen size measurements, a correlation analysis was performed. Since no statistical significant difference was found, it is fair to conclude that the collateral vein lumen size is not correlated with timespan between initial DVT and treatment. Furthermore, the number of DVTs did not seem to be related to higher collateral vein lumen size measurements. Moreover, with a preinterventional DVT recurrence of $27 \%$ it may be suggested that these patients had a higher predisposition for thrombotic stent occlusion. ${ }^{23}$ However, no significant difference in stent failure after multiple DVTs could be found $(P=0.41)$.

Previous literature describes that longer stented tracts are more prone to stent occlusion. ${ }^{23-25}$ This might be caused by a limited flow in the venous system combined with a high thrombogenicity to stent material. However, in this study, no significant evidence $(P=0.89)$ was found for the rate of stent occlusion and extent of diseased vein segments.

The results in this study might postulate about implications for pre-procedural imaging, treatment strategies and post-procedural strategies. Currently, crosssectional imaging is used to determine the extent and severity of deep venous obstruction. Moreover, it might distinguish patients fit for a simple endovascular procedure and those who are more likely to be favored by a more extensive intervention. However, based on the results that indicate a probable role for collateral vein diameter and success of deep venous intervention, future research should focus on this item into more detail.

Although it was not possible to determine a single cut-off value above that all interventions would fail, a trend could be shown. In practice, it should be wondered, when not treating a patient due to the high risk of thrombotic stent occlusion outweighs possible intervention related complications. Furthermore, it should be questioned whether patients with higher ROIs should be treated in a different manner than patients with smaller ROls. Theoretically, covered stents, overlaying at the orifice of the prominent collaterals, might be an adequate alternative to force the flow through the stents. Furthermore, several other options like embolization of large collaterals or rheological endeavours might seem effective as well, although occluding the collaterals might as a consequence increase the clinical complaint after stent occlusions due to other causes. Therefore, this research does not support any such procedure as long as the exact pathophysiological mechanisms are scrutinized. 
Furthermore, with stent occlusion being such a multifactorial process, multiple probable confounding factors should be eliminated as much as possible. Although the extent of post-thrombotic changes did not relate to statistically significant differences in stent patency, this should be included in future trials.

\section{Study limitations}

Due to the retrospective analysis of this research, some factors cannot be taken into account. One of which, the anticoagulation is an important issue especially during the first few weeks as to prevent thrombotic stent occlusion. In the Netherlands, specialized institutes are responsible for the regulation of INR levels. Since specialized thrombosis institutes regulate the INR levels, INR fluctuations between follow up visits are not detected by the treating physician. A cohort analysis is currently started to focus on this limitation and examine the fluctuations in relation to stent occlusion.

Besides, there are few stent occlusions in this cohort which may increase some bias. Other potential confounding factors like in- and outflow, iliac confluence stenting technique, venous pressure measurements, diameter of stents, pelvic collaterals and technical aspects, should be taken into account in a prospective trial. Additionally, MR evaluation after stenting should be performed to follow collateral vein lumen sizes after stenting.

Likewise, the diversion and percentage of blood flow to guarantee patent stents, remain a difficult part to analyze since a quantified parameter is still lacking. If these parameters are quantified and compared before, during and after stenting they should provide valuable information about the future outcome. Since these factors are currently unavailable, additional images techniques do show the importance of collaterals as presented in this article and should be focused into more detail.

\section{Conclusion}

The collateral vein lumen size of major venous collateral pathways seems to be related to stent occlusion in deep venous interventions. This preliminary observation might have a significant impact on pre-interventional imaging, treatment strategies and post-interventional protocols. Moreover, these findings may guide future research into the significance of collaterals in deep venous obstructions. 


\section{References}

1. Cohen AT, Agnelli G, Anderson FA, Arcelus JI, Bergqvist D, Brecht JG, et al. Venous thromboembolism (VTE) in Europe. The number of VTE events and associated morbidity and mortality. Thromb Haemost. 2007;98(4):756-64.

2. Naess IA, Christiansen SC, Romundstad P, Cannegieter SC, Rosendaal FR, Hammerstrom J. Incidence and mortality of venous thrombosis: a population-based study. J Thromb Haemost. 2007;5(4):692-9.

3. Mohr DN, Silverstein MD, Heit JA, Petterson TM, O'Fallon WM, Melton LJ. The venous stasis syndrome after deep venous thrombosis or pulmonary embolism: a population-based study. Mayo Clin Proc. 2000;75(12):1249-56.

4. Rabinovich A, Kahn SR. The postthrombotic syndrome: current evidence and future challenges. J Thromb Haemost. 2017 Feb; 15 (2): 230-41.

5. Carman TL. Prevention of the Post-Thrombotic Syndrome. Curr Treat Options Cardiovasc Med. 2016;18(8):51.

6. Comerota AJ, Oostra C, Fayad Z, Gunning W, Henke P, Luke C, et al. A histological and functional description of the tissue causing chronic postthrombotic venous obstruction. Thromb Res. 2015;135(5):882-7.

7. Piciucchi S, Barone D, Sanna S, Dubini A, Goodman LR, Oboldi D, et al. The azygos vein pathway: an overview from anatomical variations to pathological changes. Insights Imaging. 2014;5(5):619-28.

8. Umeoka S, Koyama T, Togashi K, Kobayashi H, Akuta K. Vascular dilatation in the pelvis: identification with CT and MR imaging. Radiographics. 2004;24(1):193-208.

9. Labropoulos N, Borge M, Pierce K, Pappas PJ. Criteria for defining significant central vein stenosis with duplex ultrasound. J Vasc Surg. 2007;46(1):101-7.

10. Arnoldussen $\mathrm{CW}$, de Graaf $\mathrm{R}$, Wittens $\mathrm{CH}$, de Haan MW. Value of magnetic resonance venography and computed tomographic venography in lower extremity chronic venous disease. Phlebology. 2013;28 Suppl 1:169-75.

11. Arnoldussen $\mathrm{CW}$, Toonder I, Wittens $\mathrm{CH}$. A novel scoring system for lower-extremity venous pathology analysed using magnetic resonance venography and duplex ultrasound. Phlebology. 2012;27 Suppl 1:163-70.

12. Marston W, Fish D, Unger J, Keagy B. Incidence of and risk factors for iliocaval venous obstruction in patients with active or healed venous leg ulcers. J Vasc Surg. 2011;53(5):1303-8.

13. Tick LW, Kramer MH, Rosendaal FR, Faber WR, Doggen CJ. Risk factors for post-thrombotic syndrome in patients with a first deep venous thrombosis. J Thromb Haemost. 2008;6(12):2075-81.

14. Akesson H, Brudin L, Dahlstrom JA, Eklof B, Ohlin P, Plate G. Venous function assessed during a 5 year period after acute ilio-femoral venous thrombosis treated with anticoagulation. Eur J Vasc Surg. 1990;4(1):43-8.

15. Wittens C, Davies AH, Baekgaard N, Broholm R, Cavezzi A, Chastanet S, et al. Editor's Choice Management of Chronic Venous Disease: Clinical Practice Guidelines of the European Society for Vascular Surgery (ESVS). Eur J Vasc Endovasc Surg. 2015;49(6):678-737.

16. Wen-da W, Yu Z, Yue-Xin C. Stenting for chronic obstructive venous disease: A current comprehensive meta-analysis and systematic review. Phlebology. 2016;31(6):376-89.

17. Murphy EH, Johns B, Varney E, Raju S. Endovascular management of chronic total occlusions of the inferior vena cava and iliac veins. J Vasc Surg Venous Lymphat Disord. 2017;5(1):47-59.

18. van Vuuren $T$, de Wolf MAF, Arnoldussen C, Kurstjens RLM, van Laanen JHH, Jalaie $H$, et al. Reconstruction of the femoro-ilio-caval outflow by percutaneous and hybrid interventions in symptomatic deep venous obstruction. Eur J Vasc Endovasc Surg. 2017.Oct; 54 (4): 495-503.

19. Kurstjens RL, van Vuuren TM, de Wolf MA, de Graaf R, Arnoldussen CW, Wittens $\mathrm{CH}$. Abdominal and pubic collateral veins as indicators of deep venous obstruction. J Vasc Surg Venous Lymphat Disord. 2016;4(4):426-33.

20. Markel A, Meissner M, Manzo RA, Bergelin RO, Strandness DE, Jr. Deep venous thrombosis: rate of spontaneous lysis and thrombus extension. Int Angiol. 2003;22(4):376-82.

21. Lurie F. Invited commentary. Journal of Vascular Surgery: Venous and Lymphatic Disorders.5(1):95. 
22. Neglen P, Raju S. Intravascular ultrasound scan evaluation of the obstructed vein. J Vasc Surg. 2002;35(4):694-700.

23. Neglen P, Raju S. In-stent recurrent stenosis in stents placed in the lower extremity venous outflow tract. J Vasc Surg. 2004;39(1):181-7.

24. Neglen P, Hollis KC, Olivier J, Raju S. Stenting of the venous outflow in chronic venous disease: longterm stent-related outcome, clinical, and hemodynamic result. J Vasc Surg. 2007;46(5):979-90.

25. Neglen P, Tackett TP, Jr., Raju S. Venous stenting across the inguinal ligament. J Vasc Surg. 2008;48(5):1255-61. 


\section{Chapter 8}

Illusory angiographic signs of significant iliac vein compression in healthy volunteers. Venous angiography in healthy volunteers 


\section{Abstract}

\section{Objective}

Iliac vein compression syndrome can cause severe leg symptoms. In clinical practice, it remains a challenge to differentiate which compression is clinically relevant. The aim of the current study was to assess the general treatment indications and the prevalence of angiographic signs of iliac vein compression in a group of healthy participants.

Design

Prospective cohort study

\section{Materials and methods}

A total of 20 healthy volunteers (median age 21 (20-22)) were recruited through advertisement and underwent angiography of the iliac veins. When no compression signs were present, a balloon occlusion was performed. Additionally, a 10-item survey was developed and sent to specialists treating patients with deep venous obstruction.

\section{Results}

In $16(80 \%)$ participants, at least two signs indicative of May-Thurner compression were seen. In $3(15 \%)$ subjects, narrowing of the CIV without collaterals was shown and $1(5 \%)$ did not show any signs of obstruction. A survey regarding indications for venous stenting was performed among 30 vascular specialists. In $23(70 \%)$ of the responders, collaterals were found to be the most typical sign indicative of significant venous obstruction. An angiographic sign of $>50 \%$ compression was found to be an indication to stent in $55 \%$ of responders.

\section{Conclusion}

This study demonstrates a remarkable high percentage of generally accepted signs of significant iliac vein obstruction (May-Thurner compression) on venography in healthy young subjects. Diagnosis of true iliac vein obstruction remains a major challenge, which mostly leans on improvement of clinical symptoms rather than imaging findings. Treating the patient rather than treating the picture seems to be a valid principle all the more. 


\section{Introduction}

May-Thurner anatomy is described as a left-sided common iliac vein compression (LCIV) by the right common iliac artery (RCIA) against the vertebral column. ${ }^{1}$ If this outflow obstruction gives rise to hemodynamically significant changes in blood flow, patients can experience symptoms like oedema, pain or venous skin changes, known as the May-Turner syndrome. ${ }^{2}$ When a unilateral oedema up to the upper leg is present in combination with radiographic findings, May-Thurner syndrome (MTS) is present. These radiographic signs indicative of iliac vein compression have previously been described as $>50 \%$ compression of the common iliac vein, lumen deformity of the common iliac vein or translucency, i.e. visual impression of an overriding common iliac artery on multiplane angiography. ${ }^{3}$ One of the most prominent signs of significant obstruction is the presence of collateral veins on angiography and is often referred to as an indication for treatment. ${ }^{4}$

When radiographic signs and clinical complaints are apparent, a balloon dilatation and stenting can be performed and is becoming an accepted treatment for this group of patients. ${ }^{5-7}$ However, in clinical practice, it remains difficult to predict which patients will benefit from interventional therapy.

This brings us to the question, which degree of compression is significant and which clinical signs are truly indicative of a significant compression. A compressed LCIV by the RCIA is described as an anatomic variant with prevalence up to $22-32 \%$ in cadaver research. ${ }^{8,9}$ However, the natural frequency in the general population and the clinical significance in asymptomatic subjects are still unknown. ${ }^{9-11}$

The aim of the current study was to assess the prevalence of angiographic signs of iliac vein compression in a group of healthy participants. Moreover the opinions on treatment indications for iliac vein compression was analyzed in a selected group of clinicians.

\section{Materials and methods}

This was a diagnostic study in healthy subjects to assess the prevalence of angiographic signs of iliac vein compression syndrome and assess the prevalence of collaterals. Furthermore, clinicians treating deep venous pathology (that is vascular surgeons and interventional radiologists), with varying degrees of experience were asked which radiographic signs would lead as treatment indications.

\section{Healthy participants}

A total of 20 participants were voluntary included between January 2016 and February 2017. All subjects were healthy individuals, between the ages of 18- and 45 
years, recruited by local advertisement at the University of Maastricht. Due to the higher prevalence of May-Thurner compression in previous research, a higher number of females were included in this study.

Before inclusion, a screening was performed. Subjects were screened for venous disease based on the scoring of highest $C$ of CEAP classification and Venous Clinical Severity Score (VCSS). A CEAP score of C2 or higher and a VCSS score of 2 or higher were regarded as exclusion criterium. Other exclusion criteria were arterial disease, bleeding or clotting disorders, history of deep venous thrombosis or surgery of the groin, lower limb or abdomen. Active malignancy or allergy to lidocaine or contrast agents were deemed exclusion criteria as well. To exclude pregnancy, all female participants were tested before intervention.

\section{Angiography}

Firstly, the common femoral vein of the left side was analyzed using a duplex ultrasound (MyLab Alpha, Esaote, Genoa, Italy; ProSound Alpha 7 Premier machine, Hitachi Aloka, Tokyo, Japan). When the common femoral vein and more central veins were free of post thrombotic changes, angiography images were made.

Participants received $10 \mathrm{ml} 1 \%$ local lidocaine anaesthesia around the common femoral vein area. With ultrasound guidance, the common femoral vein was punctured and a 6 Fr or 8 Fr $11 \mathrm{~cm}$ long Prelude sheat (Merit Medical, South Jordan, USA) was introduced with radiographic assistance (AlluraClarity, Philips Medical Systems, Eindhoven, the Netherlands). Access to the CFV was chosen with the tip of the sheath positioned just below the level of the inguinal ligament. Next, a guidewire was passed through this sheath into the external and common iliac vein up to the inferior vena cava. Then, $5 \mathrm{ml}$ iodinated contrast Ultravist 300 (Bayer HealthCare LLC, Whippany, New Jersey) in a solution of $5 \mathrm{ml}$ saline was injected(via the introducer sheath) in the common femoral vein and an angiography was performed with a run of 5 seconds. The injection rate was similar to our protocol and previous venous interventions with a volume of $10 \mathrm{ml}$ and flow of $5 \mathrm{ml} /$ second.

The common iliac vein was evaluated for signs indicative for May Thurner compression. Specific attention was given to analyse whether collateral flow was present on angiography images. Secondly, it was analysed whether $>50 \%$ compression of the common iliac vein (1 item), lumen deformity of the common iliac vein (1 item) or translucency, i.e. visual impression of an overriding common iliac artery (1 item) was present on multiplane angiography. The compression of the common iliac vein was estimated based on the closest proximal and closest distal point of the common iliac vein and was compared to the contralateral side whenever necessary.

When only one of these factors were present, an adequately sized balloon $(20 \mathrm{~mm}$ $22 \mathrm{~mm} \times 40 \mathrm{~mm}$, MaxiLD, Cordis, Johnson \& Johnson, Diegem, Belgium) was inflated to occlude the CIV and a second angiography was performed with $5 \mathrm{ml}$ iodinated 
contrast solution dilated with $5 \mathrm{ml}$ saline and a run of 5 seconds. The balloon was sized according to the experience of the performing radiologist and needed to create a complete occlusion of the common iliac vein. Specific attention was given to analyse whether previously mentioned factors, indicative of May-Thurner compression, were apparent when occluding the CIV. All angiographic images were analyzed after an anterior-posterior and lateral run. With balloon occlusion, an additional third run was performed in anterior-posterior projection.

After this evaluation, the balloon and sheath were removed and the insertion site in the groin was compressed for 5 minutes to obtain hemostasis. A bandage was placed and the subject was observed for 2 hours.

\section{Survey}

As to gain insight in signs which are generally believed related to deep venous obstruction (DVO), a 10-item survey was developed and sent to specialists treating patients with DVO.(Appendix 8.1) Questions regarding prominent signs of significant iliac vein compression were asked. Also, venous specialists were questioned what specific characteristics seen on angiography or IVUS were indicative to deploy a stent. This research was approved by the medical ethical committee of the Maastricht University Medical Center ( reference number 152043 (SL_2015_356)). All participants gave written consent before participation.

\section{Statistics}

The Shapiro-Wilk test was used to test the normality of the distribution. All normally distributed continuous data are presented as average with their standard deviation. Non-normally distributed data are presented as median values with inter quartile ranges. Categorical data are shown as frequencies and percentages.

Statistical analysis was performed with IBM SPSS version 23.0 software for Windows (IBM Corporation, Armonk, NY, USA).

\section{Results}

\section{Participants}

The median age of all included healthy participants was 21 years (20-22). Most of them were female $(n=16,80 \%)$ and the median BMI was $22.5(20.9-24.2) \mathrm{kg} / \mathrm{m}^{2}$

Four of the included subjects had a CEAP C1, other subjects had no signs of venous disease. None of the included females had a pregnancy in the past. Table 8.1 shows the demographics of all subjects. 
Table 8.1 Demographics of included subjects.

\begin{tabular}{lc}
\hline Age, yr (median, IQR) & $21(20-22)$ \\
Gender & $16(80)$ \\
Females (n,\%) & $4(20)$ \\
Males (n,\%) & $0(0)$ \\
Venous history(n,\%) & $0(0)$ \\
Smoking (n,\%) & $0(0)$ \\
Abdominal wall collateral (n,\%) & \\
CEAP & $16(80)$ \\
CO (n, \%) & $4(20)$ \\
C1 (n,\%) & $0(0)$ \\
$>C 2(n, \%)$ & \\
VCSS & $20(100)$ \\
$0-1(n, \%)$ & $0(0)$ \\
$\geq 2(n, \%)$ &
\end{tabular}

Yr: year, N:number, CEAP: Clinical, Etiological, anatomic, and pathophysiological, VCSS: Venous Clinical Severity Score.

In 16 (80\%) participants, the angiography showed at least two signs indicative of MayThurner compression on angiography (Example as Figure 8.1). In the remaining subjects, 3 (15\%) showed narrowing of the CIV without collaterals and 1 (5\%) did not show any signs of obstruction on angiography.
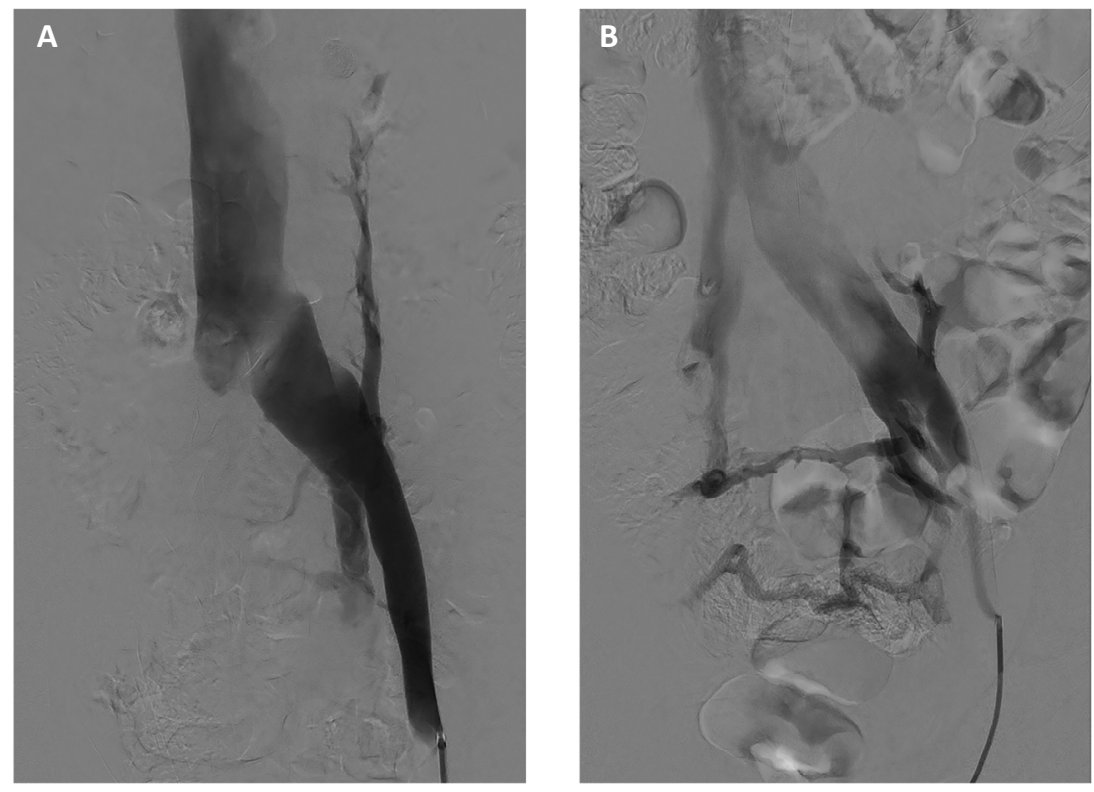

Figure 8.1 A. Venography with typical overriding right common iliac artery with translucency and flattening of the left common iliac vein, as well as paralumbar ascending and internal iliac vein collaterals. B. Venography showing translucency of the left common iliac vein caused by the overriding left common iliac artery with typical crossover collateral internal iliac vein collaterals filling the contralateral iliac veins. 
In these 4 subjects a balloon occlusion was performed with a median occlusion time of 55.5 (21-90) seconds. During the balloon inflation, all showed total crossover of collaterals on angiography (Example as Figure 8.2).

Interesting differences between males and females were noticed.

Although, 15 (94\%) females showed at least two signs of iliac vein obstruction on angiography, all 3 included male participants received additional balloon occlusion as none or one angiographic May-Thurner sign were present.Following this balloon occlusion, all subjects showed collaterals.
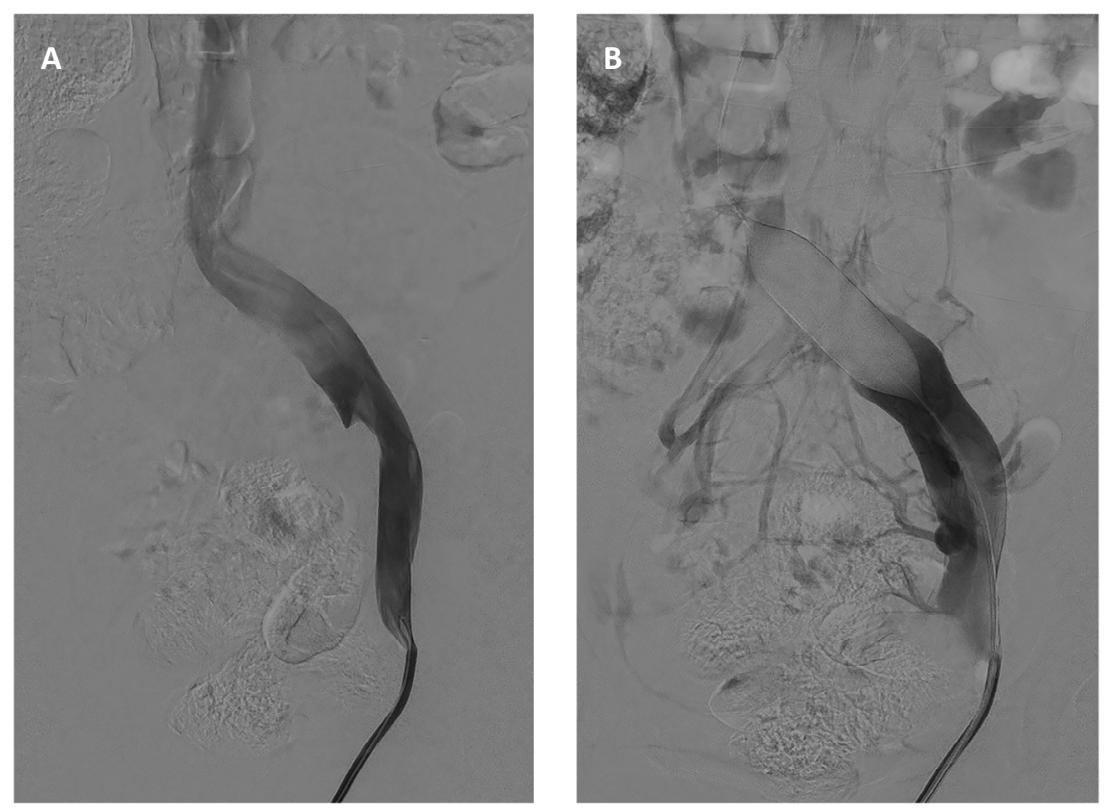

Figure 8.2 A. Venography showing fast flow through the iliac veins without signs of iliac vein compression. B. Venography during balloon occlusion of the common iliac vein showing filling of multiple deep pelvic veins, typically mentioned as collaterals present in DVO patients.

\section{Survey}

A survey regarding indications for venous stenting was performed among 30 vascular specialists with varying experience in deep venous interventions. In $63 \%$ of cases, specialist were not able to use IVUS in their hospital but did answer questions regarding IVUS use and stent indications. In 23 (70\%) of the responders, collaterals were found to be the most typical angiographic sign indicative of significant venous obstruction. An angiographic sign of $>50 \%$ compression of the CIV was found to be an indication to stent in $55 \%$ of responders. In combination with $>50 \%$ compression of 
the CIV on IVUS this was found to be a treatment indication in $69 \%$. When the sole use of IVUS would show a compression of the CIV of $30-50 \%$ it was thought to be an indication to stent in only 1 respondent. Whenever collateral veins were apparent as well, $50 \%$ would opt for stenting a patient.

With apparent lumen deformity of the CIV, $63 \%$ would deploy a stent in symptomatic patients. Moreover was translucency, i.e. visual impression of an overriding common iliac artery on multiplane angiography considered an indication to stent in only $40 \%$.

\section{Discussion}

In young healthy subjects we found a remarkably high percentage of anomalous angiographic examinations of the deep pelvic veins. These angiographic signs are deemed typical for significant deep venous obstruction and a generally accepted indication for treatment, i.e. stenting. Hereby, our results expose a potential imbalance between clinical signs and imaging findings in DVO suspected patients, causing an overtreatment risk, especially for non-thrombotic iliac vein lesions (NIVL). Overtreatment should not be underestimated as previous research already showed that only $63 \%$ of patients treated for NIVL showed a clinical treatment effect. Moreover, $24 \%$ did not show a clinical response to treatment and $14 \%$ demonstrated minor deterioration of symptoms. ${ }^{12}$ Most patients diagnosed with a NIVL are relatively young with a normal life expectancy and stents are to remain in the iliac veins for decades. Maximum follow-up of iliac vein stenting does not exceed 10 years and the long-term stent integrity and consequences of design failures are not known. In the light of the recent attention for improper use of inferior vena cava (IVC) filters, and the associated patient health and medico-legal implications, thorough indication setting for deep venous stenting seems significant.

Others have described iliac vein compression in an asymptomatic patient population before. Kibbe et al. retrospectively examined iliac vein compression on non-dedicated abdominal CT scans in patients with a low risk of deep venous thrombosis. ${ }^{9}$ In this patient population, it was found that $24 \%$ of patients had greater than $50 \%$ compression and $66 \%$ had greater than $25 \%$ compression. The latter finding however has never been associated with significant DVO in the literature. Moreover, clinical signs of DVO were not specifically determined. Again on retrospectively examined CT scans, Nazzal et al. found greater than $50 \%$ stenosis of the CIV in $44.7 \%$ of their patients. ${ }^{10}$ Oguzgurt et al. confirmed that there is a considerable overlap between the degree of compression in patients with iliac vein compression syndrome and control subjects. $^{(11)}$ These studies all used CT imaging to determine the degree of iliac vein stenosis. However, due to its known limitations and lack of prospective validation 
studies CT is not recommended to identify or exclude obstructive iliac vein lesions. ${ }^{13}$ Although venography is considered the gold standard and is used mostly in DVO diagnosis, there have been no reports of the incidence of iliac vein compression assessed with venography. ${ }^{13}$ Several signs of significant venous obstruction have been described and venography has been versatile in indicating significant lesions. One of the most eloquent reports on the pathogenicity of NIVLS was published by Raju and Neglen in 2006. Convincingly, specific findings on angiography were suggested to be associated with significant deep venous obstruction, i.e. iliac vein compression. These included contrast translucency, lumen deformity as well as axial, transpelvic, or ascending lumbar collaterals. ${ }^{3}$ We found at least two of these signs in 16/20 (80\%) of the examined healthy subjects, while another three showed one of the aforementioned signs. Altogether, this means that if only venography is taken into consideration, 19/20 (95\%) of our healthy subjects would be offered an intervention when evaluated by internationally accepted standards. In order to substantiate this assumption, we planned a survey among 30 vascular specialists with varying experience in deep venous interventions. Interestingly, in 23 (70\%) of the responders, collaterals were found to be the most typical angiographic sign indicative of significant venous obstruction. Signs like $>50 \%$ compression on angiography was found to be an indication to stent in $55 \%$ of responders. In combination with IVUS this was found to be a treatment indication in $69 \%$.

Intravascular ultrasound (IVUS) has been suggested to have additional value in the diagnosis and treatment of DVO. Neglen et al. mentioned before that IVUS was capable to identify subtle intravascular changes like webs and spurs that would stay obscure during single plane venography. ${ }^{3}$ In addition, the recent VIDIO study showed that IVUS is superior to multiplane angiography to identify iliac vein stenotic lesions. ${ }^{14}$ IVUS was not used in our study because of additional costs and invasiveness. In any case, percutaneous transfemoral or transpopliteal venography still is the gold standard for evaluating deep venous obstructions. Moreover, IVUS is costly and still not routinely used by interventionalists throughout the world. On the other hand, it would be just as unlikely for IVUS to show a non-significant lesion in case of positive angiographic signs. In the VIDIO study, a "false positive angio" was only seen in $3 \% .{ }^{14}$ The incidence of "true" false positive angiographic examinations remained unclear however, since no correlation to clinical signs of deep venous obstruction was given.

With the uncertainty in diagnostic examinations expressed in our study, clinical evaluation of DVO suspected patients becomes increasingly important. However, clinical scoring and grading systems have also become the focus of criticism lately, mainly because of the lack of validation for DVO. Others scales focus primarily on chronic venous disease in general and include the CEAP (Clinical, Etiological, anatomic, and pathophysiological) classification and Venous Clinical Severity Score (VCSS). These scores are actually developed to evaluate venous insufficiency and are not validated to take DVO into account. In a large number of patients with iliac vein compression, 
the CEAP score is low and will not change, impairing to be able to follow the effect of treatment over time. The VCSS most recently updated by Vasquez et al., combines factors of compression therapy, number of ulcers, and the CEAP scale to assess venous disease. ${ }^{15}$ The VCSS is based on 9 clinical signs and only 1 clinical symptom. ${ }^{16}$ Although reproducibility has been described to be good, this seems to be of varying degree in clinical practice. Besides, with exception to venous ulcers, visible skin changes do not show the seriousness of clinical complaints a patient can experience. Specifically, clinical subjective signs which result into troublesome daily practice should be taken into account. Venous claudication is one such item which should be validated to acquire the impact of complaints on a patients' life. An accurate, reliable and reproducible clinical scoring system should be developed to associate consequences.

In our study protocol, we included a balloon provocation test to exclude significant collateral pathways in subjects with a "false-negative" venography due to the inactive state and supine position. Surprisingly, the one subject with a negative venography showed pre-sacral and ascending paralumbar collaterals while a large balloon was inflated in the left CIV. The significance of this finding remains unclear, however may resemble normal venous collateral pathways in the pelvis.

The current study is limited by its small sample size. However, considering the overwhelming presence of positive angiographic signs in our asymptomatic population, it would not have been ethical to expose additional healthy subjects to this invasive examination. Larger studies with healthy subjects researching the longterm consequences of iliac vein stenosis in both males and females might be interesting though. It has been suggested before that compression of the iliac vein may be a risk factor for acute DVT. Due to the associated detrimental consequences like pulmonary emboli and death it may be postulated that preventative treatment in severe cases of asymptomatic iliac vein compression is acceptable. In fact, Raju et al. quite early suggested that NIVLs in an asymptomatic population may be permissive of future development of DVO. ${ }^{3}$ Based upon our results, pursuing this assumption without further knowledge into this entity might be premature to say the least. This should be included in current guidelines \{Wittens, 2015 \#22\} and clinical signs should be assessed in a structured manner in combination with angiographic findings.

\section{Conclusion}

This study showed a remarkable high percentage of generally accepted signs of significant iliac vein obstruction (May-Thurner compression) on venography in healthy young subjects. Diagnosis of true MTS remains to be a major challenge, which mostly leans on improvement of clinical assessment rather than imaging findings. Treating the patient rather than treating the picture seems to be a valid principle all the more. 


\section{References}

1. May R, Thurner J. The cause of the predominantly sinistral occurrence of thrombosis of the pelvic veins. Angiology. 1957;8(5):419-27.

2. Donatella N, Marcello BU, Gaetano V, Massimo P, Massimo M, Giancarlo B. What the Young Physician Should Know About May-Thurner Syndrome. Transl Med UniSa. 2015;12:19-28.

3. Raju S, Neglen P. High prevalence of nonthrombotic iliac vein lesions in chronic venous disease: a permissive role in pathogenicity. J Vasc Surg. 2006;44(1):136-43.

4. Kurstjens RL, de Wolf MA, van Laanen JH, de Haan MW, Wittens $\mathrm{CH}$, de Graaf R. Hemodynamic significance of collateral blood flow in chronic venous obstruction. Phlebology. 2015;30(1 Suppl): 27-34.

5. Wen-da W, Yu Z, Yue-Xin C. Stenting for chronic obstructive venous disease: A current comprehensive meta-analysis and systematic review. Phlebology. 2016;31(6):376-89.

6. Seager MJ, Busuttil A, Dharmarajah B, Davies AH. Editor's Choice-- A Systematic Review of Endovenous Stenting in Chronic Venous Disease Secondary to Iliac Vein Obstruction. Eur J Vasc Endovasc Surg. 2016;51(1):100-20.

7. Razavi MK, Jaff MR, Miller LE. Safety and Effectiveness of Stent Placement for Iliofemoral Venous Outflow Obstruction: Systematic Review and Meta-Analysis. Circ Cardiovasc Interv. 2015;8(10): e002772.

8. Brinegar KN, Sheth RA, Khademhosseini A, Bautista J, Oklu R. Iliac vein compression syndrome: Clinical, imaging and pathologic findings. World J Radiol. 2015;7(11):375-81.

9. Kibbe MR, Ujiki M, Goodwin AL, Eskandari M, Yao J, Matsumura J. Iliac vein compression in an asymptomatic patient population. J Vasc Surg. 2004;39(5):937-43.

10. Nazzal M, El-Fedaly M, Kazan V, Qu W, Renno AW, Al-Natour M, et al. Incidence and clinical significance of iliac vein compression. Vascular. 2015;23(4):337-43.

11. Oguzkurt L, Ozkan U, Ulusan S, Koc Z, Tercan F. Compression of the left common iliac vein in asymptomatic subjects and patients with left iliofemoral deep vein thrombosis. J Vasc Interv Radiol. 2008;19(3):366-70.

12. van Vuuren $T$, de Wolf MAF, Arnoldussen C, Kurstjens RLM, van Laanen JHH, Jalaie $H$, et al. Reconstruction of the femoro-ilio-caval outflow by percutaneous and hybrid interventions in symptomatic deep venous obstruction. Eur J Vasc Endovasc Surg. 2017.

13. Wittens C, Davies AH, Baekgaard N, Broholm R, Cavezzi A, Chastanet S, et al. Editor's Choice Management of Chronic Venous Disease: Clinical Practice Guidelines of the European Society for Vascular Surgery (ESVS). Eur J Vasc Endovasc Surg. 2015;49(6):678-737.

14. Gagne PJ, Tahara RW, Fastabend CP, Dzieciuchowicz L, Marston W, Vedantham S, et al. Venography versus intravascular ultrasound for diagnosing and treating iliofemoral vein obstruction. J Vasc Surg Venous Lymphat Disord. 2017;5(5):678-87.

15. Vasquez MA, Rabe E, McLafferty RB, Shortell CK, Marston WA, Gillespie D, et al. Revision of the venous clinical severity score: venous outcomes consensus statement: special communication of the American Venous Forum Ad Hoc Outcomes Working Group. J Vasc Surg. 2010;52(5):1387-96.

16. Marston WA, Vasquez MA, Lurie F, Wakefield TW, Rabe E, Shortell CK, et al. Multicenter assessment of the repeatability and reproducibility of the revised Venous Clinical Severity Score (rVCSS). J Vasc Surg Venous Lymphat Disord. 2013;1(3):219-24. 


\section{Appendix 8.1}

How many pateints suspective of MTS did you treat the last 12 months?

Do you consider $>50 \%$ compression of CIV on phlebography indication to stent?

Do you considering pancakeing of CIV on phlebography an indication to stent?

Do you consider translucency, i.e. visual impression of an overriding common iliac artery

Do you consider collateral venous flow on phlebography as an indication to stent MTS?

Do you consider $>50 \%$ compression of the common iliac vein on IVUS as an indication to stent MTS?

Do you consider $30-50 \%$ compression of the common iliac vein on IVUS as an indication to stent MTS?

yes

Do you consider 30-50\% compression of the common iliac vein AND collateral venous flow on IVUS as an indication to stent MTS? 


\section{Chapter 9}

The Self-Assessed Venous Severity questionnaire for monitoring symptoms of deep venous obstructions 


\section{Abstract}

\section{Introduction}

Clinical complaints related to deep venous obstructions can be evaluated by several scoring systems. Unfortunately, there is currently no uniform disease specific clinical outcome measurement for the whole spectrum of venous disease. A self-assessed venous severity questionnaire was developed to analyse whether patients were able to score objective and subjective clinical symptoms.

\section{Methods}

This cohort study consisted questions, including a visually guided Villalta scale, VCSS score, the VEINES QOL/Sym, and questions regarding venous claudication (VC). Patients filled out the questionnaire (Q1), and a random subset of participants was asked to complete a second questionnaire within 1-7 days (Q2). The treating physician and a trained researcher performed the same.

\section{Results}

In total, 64 patients were included, and 39 completed a Q2 questionnaire. The VCSS reliability was highest in the physician group with an interclass correlation of $0.75, P<0.01$ and a reliability of $0.36 P=0.008$ in $Q 1$ versus $Q 2$.

The self-assessed Villalta scale showed a reliability of $\mathrm{k} 0.04$ when compared to the physicianreported scales. The patients Q1 and Q2 revealed a Villalta scale of $\kappa$ 0.48, $P<0.01$.

The VEINES/QOL scores and clinical scores showed the highest correlation between Q1 versus Q2. The venous claudication questions did show a very high correlation with the VEINES/QOL of $-0.97 P<0.01$.

\section{Conclusion}

A self-assessed venous severity questionnaire (SAVS-Q) can be a promising basic tool in the evaluation of venous obstruction. The patients' inter-class correlation shows best reproducibility in the evaluation of clinical complaints. A new venous instrument should be developed in order to increase reproducibility. 


\section{Introduction}

During the past decades, there has been a rapid increase in available treatments for venous disease. Nowadays, it is not merely technical success but a combination of technical and patient-experienced outcomes, socioeconomic costs, and the clinical evaluation of complaints that represents the effectiveness of a treatment. This evaluation of clinical complaints related to deep venous pathology can be performed by utilizing various psychometric scoring instruments, both clinical and patientreported. ${ }^{1,2}$ Unfortunately, there is currently no uniform disease-specific outcome measure for the whole spectrum of deep venous pathology. Therefore, we need to rely on the available scoring systems while gaining consensus. So far, the Venous Clinical Severity Score (VCSS) has been described as a well-known scoring item for venous pathology. ${ }^{3,4}$ Additionally, the Villalta scale was recommended by the Committee of International Society on Thrombosis and Haemostasis for patients with a post thrombotic syndrome (PTS). ${ }^{5}$

The main disadvantage of currently available instruments is the fact that they mainly focus on physician-related outcomes. ${ }^{2}$ The patients' experience and quality of life (QOL), which can be comparable to patients with pectoral angina or pulmonary disease, ${ }^{6}$ are not taken into account. Currently, clinical measurements are performed by anamnestic questions filled out by the physician; the interpretation of these outcomes is often colored by the physician's opinion and the limited recollection abilities of the patient. Besides, the physician may undervalue reported complaints, not sensing a patient's suffering. ${ }^{7}$ For this reason, using patient-reported outcomes to analyze the patient's perspective on complaints and the effect of treatment is very important. Ultimately, this is the best way to assess complaints and symptoms during a patient's daily routine.

Thus far, the available scoring systems show conflicting evidence regarding the validity, reliability and reproducibility of longitudinal results. ${ }^{1,8}$ Furthermore, none of the abovementioned scoring systems analyzes venous claudication, which can tremendously affect daily activities.

Ideally, the patient should be trained to score all subjective and objective items in order to increase reproducible and uniform results. In order to address this, we developed a self-assessed venous severity questionnaire evaluating the abovementioned shortcomings. Hence, the aim of this questionnaire was to analyze whether patients were able to score objective and subjective clinical symptoms and signs. Subsequently, we tested whether the data-entry was performed in a consistent and reproducible way. 


\section{Methods}

This cohort study was approved by the ethical committee of the Maastricht University/AzM (METC 16-4-80). All patients were requested to sign an informed consent and they approved participation.

Participants were recruited at the vascular surgery outpatient clinic of the Maastricht University Medical Centre (MUMC). Subjects were eligible for inclusion when visiting the hospital with complaints related to venous pathology. The outpatient clinic visit could be related to a follow up control or a first visit without prior deep venous interventions.

Exclusion criteria were a deep venous thrombosis (DVT) less than 6 months previously or surgery 3 months prior to the visit, as this could influence the interpretation of some questions. Other exclusion criteria were an age of less than 18 years or an inability to read and answer a Dutch questionnaire.

\section{Questionnaire}

The survey included a total of 99 questions. All questions were translated into the Dutch language and understandable patient parlance. Before implementation, the comprehension of all included questions was tested by three non-professionals with different levels of educational background.

The self assessed venous questionnaire (SAVS-Q) included a visually guided Villalta scale $^{5}$ and a venous clinical severity score (VCSS). Additionally, both the VEINES QOL/Sym, SF-36v2 and questions regarding venous claudication (VC) were included. VC was scored as being present when symptoms of pain developed during activity and subsided during rest or when the patient lifted the leg.

Since venous claudication is an important factor in analyzing the influence of venous disease during daily activities, it should be defined accurately. In order to reveal its effect on daily activities and provide precise insight into its presence or absence, venous claudication was evaluated with a subdivision of 4 questions, as presented in appendix 9.1. A negative answer in all questions represented an absence of venous claudication. When a patient did experience complaints but was never hampered during daily routines, this was also scored as an absence of venous claudication.

\section{Q1 and Q2}

Patients were requested to fill out all items of the questionnaire (Q1) when visiting the outpatient clinic. All participants were asked to complete this task before entering the physician's room.

Afterwards, a duplex ultrasound (DUS) was performed to search for post thrombotic scarification, search for venous insufficiency, or analyze stent patency. Subsequently, 
participants entered the physician's room to have their clinical complaints evaluated, undergo a physical examination, and discuss the results of the DUS. The treating physician was blinded to the patients' answers and filled out all Villalta, VCSS, and VC items of the index leg based on the anamnesis and physical examination.

In order to compare the consistency of the physicians' score, a trained vascular researcher performed a separate anamnesis and physical examination during the outpatient visit. Subsequently, this trained researcher was requested to answer the exact same items as the patient and the treating physician, without being aware of their answers.

To analyze the patients' consistency in answering the questions, a random subset of participants was asked to complete a second questionnaire within 1-7 days after visiting the outpatient clinic (Q2). The items in this second survey included the exact same questions as Q1 but in reversed order.

\section{Statistics}

Continuous variables are represented as mean values with their standard deviation when normally distributed. Non-normally distributed variables are represented as medians with their inter quartile range (IQR).

To calculate the consistency of answers in continuous scales, an inter- and intraobserver concordance was calculated by use of the Cohen's kappa. Agreement was expressed by the kappa ( $\mathrm{k}$ ) coefficient with a $95 \%$ confidence interval $(\mathrm{Cl})$ for ordinal scales. A $\mathrm{k}$ value of $>0.80$ represented very good/high concordance, $0.80-0.61$ good concordance, 0.60-0.41 moderate concordance, 0.40-0.21 fair concordance, and $\leq 0.20$ poor concordance.

To calculate the consistency of answers in continuous variables, an interclass correlation was calculated the Cronbach's alpha, in which a score $>0.9$ represented almost perfect correlation and $>0.7$ represented strong correlation. Correlations between the QOL and other clinical scores were analyzed by the Spearman correlation test.

A $P$-value of 0.05 was regarded as being statistically significant. Data were analyzed using IBM SPSS Statistics v23.0 (IBM Corporation, Armonk, NY, USA).

\section{Results}

A total of 78 patients were included in this study. Questionnaires with non-fully completed clinical scores were excluded $(n=14)$, as it was impossible to calculate the VCSS score, VC, or Villalta scale. In total, 64 Q1 questionnaires could be analyzed. A subset of 39 patients filled out the second questionnaire after a period of 1-7 days. Table 9.1 shows baseline characteristics of all included subjects. 
PTS was scored by the grading of the Villalta score. A subdivision was made among Villalta scores of 0-4 indicating no PTS, 5-9 indicating mild PTS, 10-14 moderate PTS and $>15$ severe PTS.

Table 9.1 Demographics of included subjects.

\begin{tabular}{|c|c|}
\hline & Outcome \\
\hline \multicolumn{2}{|l|}{ Diseased side } \\
\hline Left $(\mathrm{N}, \%)$ & $41,64 \%$ \\
\hline Rigth $(\mathrm{N}, \%)$ & $6,9 \%$ \\
\hline Bilateral $(\mathrm{N}, \%)$ & $17,27 \%$ \\
\hline \multicolumn{2}{|l|}{ Gender } \\
\hline Female $(\mathrm{N}, \%)$ & $38,60 \%$ \\
\hline Male $(\mathrm{N}, \%)$ & $26,40 \%$ \\
\hline \multicolumn{2}{|l|}{ Age } \\
\hline$y r \pm S D(\min -\max )$ & $43,9 \pm 16(18-72)$ \\
\hline
\end{tabular}

$\mathrm{N}=$ number, $\mathrm{yr}=$ year, $\mathrm{SD}=$ standard deviation, $\min =$ minimum, $\max =$ maximum .

\section{Scores of patients versus treating physician}

\section{Clinical scores}

Table 9.2 shows the median scores of the self-assessed patient scores and the physician assessed Villalta scale, VCSS, and VC. The total Villalta scores of the patients were 11.3 in Q1 and 10 in Q2. In contrast, the physician and the trained researchers scored a mean of 7 versus 9 respectively. As shown in Table 9.2, the VCSS scores of patients were higher than the VCSS physicians' scores with means of 7.4 versus 10.6 and 5.8 versus 5.9 respectively.

Table 9.2 Total scores of clinical assessment.

\begin{tabular}{lc}
\hline Subscale instrument & Outcome \\
\hline Total Villalta scale Q1 (mean $\pm S D$, min-max) & $11,3 \pm 6,8(0-25)$ \\
Total Villalta scale Q2 (median, IQR) & $10(6,25-15)$ \\
Total Villalta scale Physician 1 (mean $\pm S D$, min, max) & $7 \pm 4.0(1-21)$ \\
Total Villalta scale researcher (mean $\pm S D$, min, max) & $9 \pm 7.8(1-20)$ \\
Total VCSS Q1(mean $\pm S D$, min-max) & $7,4 \pm 6(0-22)$ \\
Total VCSS Q2(mean $\pm S D$, min-max) & $10,6 \pm 5,3(7-22)$ \\
Total VCSS Physician 1(mean $\pm S D$, min-max) & $5,8 \pm 3,1(0-12)$ \\
Total VCSS researcher(mean $\pm S D$, min-max) & $5,9 \pm 2,9(1-13)$ \\
\hline
\end{tabular}

$\mathrm{SD}=$ standard deviation, $\min =$ minimum, $\max =$ maximum .

Table 9.3 compares the physicians' scores to the patients' score showing a VCSS interclass correlation of $0.51, P<0.01$. In addition, a variability in a graded Villalta scale revealed a very low kappa score of 0.04 . 
Table 9.3 Variability of scores.

\begin{tabular}{lccc}
\hline Category & Scale & K-score & $P$-value \\
\hline Physician versus Q1 & VCSS & 0,51 & $<0.01$ \\
& VC & 0,44 & $<0.01$ \\
& Villalta & 0,04 & 0.49 \\
Physician versus researcher & VCSS & 0,75 & $<0.01$ \\
& Villalta & 0,29 & $=0.001$ \\
Q1 versus Q2 & VCSS & 0,36 & 0.008 \\
& VC & 0,95 & $<0.01$ \\
& Villalta & 0,48 & $<0.01$ \\
\hline
\end{tabular}

$\mathrm{Q} 1=$ questionnaire $1, \mathrm{Q} 2=$ questionnaire $2, \mathrm{VCSS}=$ venous clinical severity score, VC= venous claudication.

\section{Quality of life versus clinical scores}

When the VCSS scored by the physician was correlated to the VEINES/QOL, a low correlation of $0.39 P<0.01$ was found. Additionally, the graded Villalta scale scored by the physician revealed a moderate correlation with the VEINES/QOL of $-0.61, P<0.01$.

\section{Physician versus trained researcher}

\section{Clinical scores}

The VCSS reliability was highest in the physician versus trained researcher groups, with an intraclass correlation coefficient of $0.75, P<0.01$. The venous claudication was not scored by the trained researcher and could not be compared to the physician's score.

\section{Scores of patients}

\section{Clinical scores}

Table 9.3 shows that the most reproducible items were scored by the patients, whose venous claudication score revealed a kappa of $0.95, P<0.01$. Additionally, the graded PTS scale was tested, revealing a kappa of $0.48, P<0.01$. The VCSS revealed a reliability of $0.36, P=0.008$ and was least consistently scored by the patients themselves.

\section{Quality of life versus clinical scores}

Some additional analyses were performed to analyze the correlation between the VEINES/QOL and the patients' self-assessed clinical scores. The overall interclass VEINES/QOL correlation between Q1 and Q2 was 0. 95, $P<0,01(\mathrm{~N}=39)$. The Q1 Villalta scale and Q1 VEINES/QOL showed a moderate correlation of $-0.60, P<0,01$. The VCSS of Q1 could be correlated with the VEINES/QOL of Q1 in 52 cases and revealed a 
moderate to weak correlation of $-0.53, P<0,01$. Finally, the venous claudication showed a very high correlation of $-0.97 P<0.01$ with the VEINES/QOL.

Since the differences in Villalta scale were very high, all separate items were tested for consistency in scoring. Table 9.4 shows these results, which indicate that, except for venous ulcera, patients are most consistent in scoring their complaints and clinical signs.

Table 9.4 Individual items of the Villalta scale.

\begin{tabular}{lcccccc}
\hline & \multicolumn{2}{c}{ Q1-physician } & \multicolumn{2}{c}{ physician-researcher } & \multicolumn{2}{c}{$\mathrm{Q} 1-\mathrm{Q} 2$} \\
\hline & $\mathrm{K}$ & $P$-value & $\mathrm{K}$ & $P$-value & $\mathrm{K}$ & $P$-value \\
\hline Pain & 0,2 & 0,004 & 0.11 & 0.11 & 0.39 & $<0.01$ \\
Cramps & 0,31 & $<0,01$ & 0.22 & 0.007 & 0.60 & $<0.01$ \\
Heaviness & 0,15 & 0,03 & 0.36 & $<0.01$ & 0.51 & $<0.01$ \\
Paresthesia & 0,23 & 0,01 & 0.36 & $<0.01$ & 0.60 & $<0.01$ \\
Pruritis & 0,3 & $<0,01$ & 0.44 & $<0.01$ & 0.54 & $<0.01$ \\
Edema & 0,23 & 0,01 & 0.17 & 0.02 & 0.62 & $<0.01$ \\
Skin induration & 0,07 & 0,23 & 0.042 & 0.56 & 0.35 & $<0.01$ \\
Hyperpigmentation & 0,18 & 0.02 & 0.56 & $<0.01$ & 0.76 & $<0.01$ \\
Redness & 0,28 & 0.01 & 0.33 & 0.01 & 0.69 & $<0.01$ \\
Venous ectasia & 0,16 & 0.01 & 0.056 & 0.49 & 0.52 & $<0.01$ \\
Pain on calf compression & 0,17 & 0.02 & 0.26 & 0.004 & 0.61 & $<0.01$ \\
Venous ulcer & 0,25 & $<0,01$ & 1.00 & $<0.01$ & 0.57 & $<0.01$ \\
\hline
\end{tabular}

\section{Discussion}

In this study, a self-assessed venous severity questionnaire was tested to analyze whether patients were able to score objective and subjective clinical symptoms and signs. Subsequently, it was tested whether the data entry was performed in a consistent and reproducible way. As the results of this article demonstrate, patients are best able to reproducibly score symptoms for the Villalta scale and venous claudication. In contrast, physicians tend to underestimate the patients' complaints, a tendency exemplified by the fact that all of the physicians' scores are lower than the patients' scores. Moreover, physicians tend to score in a less reproducible way, as revealed by the very low concordance of kappa results between the physicians' and the trained researchers' assessments.

The VCSS score, Villalta scale, and VC were used because they are able to describe subjective and objective complaints in a way that allows longitudinal follow-up. In previous research, the Villalta scale has been described as demonstrating both a good correlation with QOL and excellent interobserver reliability. ${ }^{9}$ However, some aspects of the scale have not been validated yet, which could result in biased interrater reliability. Moreover, the main disadvantage of the Villalta scale as a scoring system is 
that it contains a total of 12 items in which a subdivision of 5 items regarding symptoms and 7 items regarding clinical signs can be made; both the physician and the patient score part of the items. For this reason, Utne et al developed an alternative questionnaire in which patients scored all items. A kappa of 0.6 was presented when the self-reported Villalta scale was used, and a kappa of 0.82 was presented when the visually assisted form was used. ${ }^{10}$ However, the research of Utne et al. did not take reproducibility of outcomes into account, as the patients filled out the questionnaire only once. Moreover, the patients' symptom score was directly translated into the physician's symptom score, which amounts to a $100 \%$ reproducibility in 5 out of 12 questions. Subsequently, the current research took these biases into account and revealed a Villalta self-assessed kappa of 0.48 .

Next, QOL was correlated to the self-assessed scores which showed a moderate to good correlation of -0.60 , indicating lower QOL with lower Villalta scores. When the QOL was compared to the physicians' Villalta score, a correlation of -0.61 was shown. In other words, both the patient and physician reported scores correlate to the QOL scores in an equal way. Moreover, when the VCSS scores of patients and physicians were correlated to the QOL, a higher correlation was noted in the patient group.

With this self-assessed venous score, an acceptable and reasonably easy-to-use monitoring tool is introduced. Based on the outcomes of this study, we recommend further extension and testing of the self-assessed venous severity score as patients are relatively consistent in scoring subjective and objective symptoms, but interrater correlations are still moderate. Specifically, when the scores are used to analyze treatment outcomes, only reproducible results should be considered.

Although slightly favorable outcomes have been presented, some limitations should be mentioned. As stated in previous research, a bias might be found because parts of the questionnaire have not been validated yet. ${ }^{11}$ For this reason, it is important to extend the results of this pilot and eventually validate the questionnaire as a new venous instrument. Subsequently, it is important to ensure that patients of different educational backgrounds and speaking different languages can fill out the questionnaire consistently. A larger group of subjects should be tested; with current pilot results, it is possible to perform a proper power calculation.

We believe that part of the low concordance between the two scoring physicians can be explained by the fact that the clinical researcher was only trained for a few weeks. A broadening of clinical knowledge could result in higher concordance and more consistency. However, these differences were not based on objective scores alone; the patients' scores proved to be consistent and might be the most reliable. 


\section{Conclusion}

A self-assessed venous severity questionnaire (SAVS-Q) can be a promising basic tool in the evaluation of venous obstruction. The patients' inter-class correlation shows best reproducibility in the evaluation of clinical complaints. A new venous instrument should be developed in order to increase reproducibility. 


\section{References}

1. Catarinella FS, Nieman FH, Wittens $\mathrm{CH}$. An overview of the most commonly used venous quality of life and clinical outcome measurements. J Vasc Surg Venous Lymphat Disord. 2015;3:333-40.

2. Wik HS, Ghanima W, Sandset PM, Kahn SR. Scoring Systems for Postthrombotic Syndrome. Seminars in thrombosis and hemostasis. 2017.

3. Meissner MH, Natiello C, Nicholls SC. Performance characteristics of the venous clinical severity score. J Vasc Surg. 2002;36:889-95.

4. Marston WA, Vasquez MA, Lurie F, Wakefield TW, Rabe E, Shortell CK, Lohr JM, Passman MA, McLafferty RB. Multicenter assessment of the repeatability and reproducibility of the revised Venous Clinical Severity Score (rVCSS). J Vasc Surg Venous Lymphat Disord. 2013;1:219-24.

5. Kahn SR, Partsch H, Vedantham S, Prandoni P, Kearon C. Definition of post-thrombotic syndrome of the leg for use in clinical investigations: a recommendation for standardization. J Thromb Haemost. 2009;7:879-83.

6. Kahn SR, Ducruet T, Lamping DL, Arsenault L, Miron MJ, Roussin A, Desmarais S, Joyal F, Kassis J, Solymoss S, Desjardins L, Johri M, Shrier I. Prospective evaluation of health-related quality of life in patients with deep venous thrombosis. Arch Intern Med. 2005;165:1173-8.

7. Hull R, Butcher P. Value of patient self-assessment in the diagnosis and monitoring of post-thrombotic syndrome: the Patient-Tracked Symptoms questionnaire. Clin Appl Thromb Hemost. 2012;18:345-50.

8. Rodger MA, Kahn SR, Le Gal G, Solymoss S, Chagnon I, Anderson DR, Wells PS, Kovacs MJ. Interobserver reliability of measures to assess the post-thrombotic syndrome. Thromb Haemost. 2008;100:164-6.

9. Catarinella FS, Nieman $\mathrm{FH}$, Wittens $\mathrm{CH}$. The relation between clinical scores and quality-of-life in longterm follow-up. Phlebology. 2016;31:99-105.

10. Utne KK, Ghanima W, Foyn S, Kahn S, Sandset PM, Wik HS. Development and validation of a tool for patient reporting of symptoms and signs of the post-thrombotic syndrome. Thromb Haemost. 2016;115:361-7.

11. Strijkers RH, Wittens CH, Kahn SR. Villalta scale: goals and limitations. Phlebology. 2012;27 Suppl 1:130-5. 


\section{Appendix 9.1 Translated from the Dutch version}

Questions regarding venous claudication (Please answer 1 item per question)

1. Do you experience pain in your leg(s) during excercise (walking, climbing stairs or during sports)?
o never
- hardly ever
o sometimes
o often
- always

2. When will the pain in your leg(s) subside immediately?

o never

o when I stop moving

o when I sit down and elevate my leg(s)

- I do not experience any pain in my leg(s)

3. How may meters/minutes can you mobilize without complaints?
- Less than 100 meter OR less than 2 minutes
- More than 100 meter, but less than 1000 meter OR 2-15 minutes
- More than 1000 meter OR > 15 minutes
- I do not experience any pain when mobilizing

4. Does your pain influence daily activities?
○ never
- hardly ever
o sometimes
o often
○ always 


\section{Chapter 10}

A randomized controlled trial comparing venous stenting with conservative treatment in patients with Deep Venous Obstruction: research protocol 


\section{Abstract}

\section{Introduction}

Deep venous obstruction (DVO) has a great impact on Quality of Life (QoL) comparable to angina pectoris or chronic pulmonary disease. Post thrombotic scar formation and May-Thurner compression syndrome (MTS) are the most common causes of DVO. Conventional treatment of deep venous obstruction focuses on reducing pain or leg swelling by use of (pain) medication and therapeutic elastic stockings. In the past, a venous bypass was offered in severe post thrombotic cases, but this procedure showed bad clinical and patency outcomes. With the introduction of percutaneous angioplasty and dedicated venous stents new opportunities were created. Deep venous stenting has been shown to be effective in retrospective case series. However, there is no prior research in which QoL after interventional treatment is compared to QoL after conventional treatment. Currently, there is a debate about the true additional value of interventional treatment. We investigate whether those patients who are treated with stenting experience a change in SF-36 and the VEINES-QoL/Sym questionnaires compared to conventionally treated patients.

\section{Methods and analysis}

This is a randomized trial comparing conservative deep venous management to interventional treatment. A total of 130 patients with post thrombotic syndrome (PTS) or MTS, eligible for interventional percutaneous treatment, who did not have previous deep venous intervention will be included. Patients will be randomized to conservative treatment or venous stenting and stratified for PTS or MTS subgroup. Conservative treatment consists of either one or a combination of pain medication, manual lymphatic drainage, compression stockings and regular post thrombotic anticoagulants therapy.

The primary outcome is the QoL change after 12 months compared to baseline QoL. Secondary outcomes are QoL changes at 6 weeks, clinical assessment of DVO, recurrence rate of DVT at 6 weeks and 12 months and the total amount of working days loss. Intervention specific outcomes include complications and patency. 


\section{Introduction}

Deep venous obstruction (DVO) is a condition caused by intraluminal or extraluminal obstructions of the veins. ${ }^{1}$ In most cases, an intraluminal obstruction is related to a previous deep venous thrombosis (DVT). Annually, about 1-2 per 1000 people in Western European countries develop a DVT, ${ }^{2,3}$ in which $40 \%$ of cases affect the iliofemoral region. ${ }^{4}$ After a DVT, the blood clot should resolve by natural recanalization of the vessel. ${ }^{5}$ However, in $59 \%$ of patients with iliofemoral DVT, the recanalization process is inadequate which leads to vein scarification and venous outflow obstruction. ${ }^{5-7}$ Subsequently, this may result in debilitating symptoms which are categorized in the Post Thrombotic Syndrome (PTS). ${ }^{8}$ These symptoms may include pain, tired legs, venous claudication, cramps, edema, hyperpigmentation or even venous ulcers. ${ }^{8,9}$

As aforementioned, the second cause of venous outflow obstruction is an extraluminal obstruction, of which the iliac vein compression syndrome is most common. The best known iliac vein compression syndrome is the May-Thurner Syndrome (MTS). ${ }^{1}$ The MTS is generally characterized by a significant compression $(>50 \%)$ of the left common iliac vein between the lumbar vertebral column and the right common iliac artery. ${ }^{10}$ (Figure 10.1) Subsequently, this causes a venous outflow obstruction which leads to venous hypertension and related symptoms like leg swelling and pain. Besides the outflow obstruction, patients with MTS may have an increased risk of developing a DVT and patients with PTS may have an increased risk of developing a recurrent because of anatomical variance and related blood stasis. ${ }^{11}$

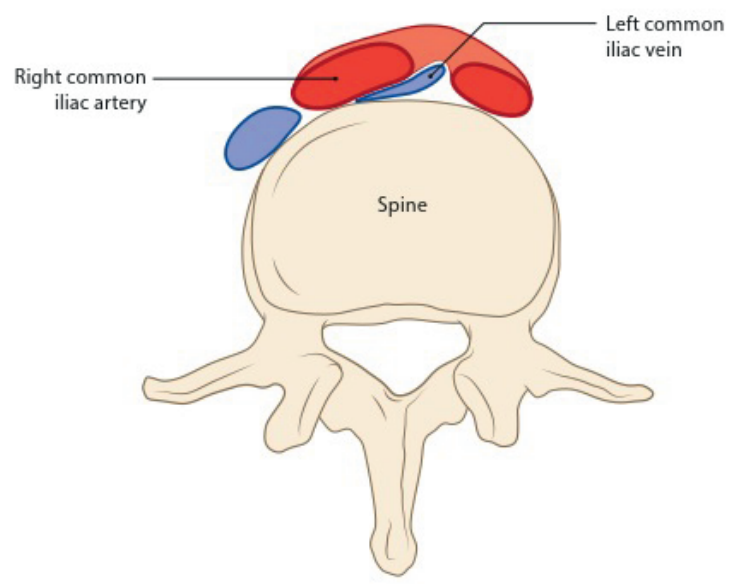

Figure 10.1 Example of May-Thurner compression. 
The component of blood stasis is encompassed in the Virchow's triad in which a hypercoagulable state, vascular wall damage and venous stasis explain the development of a deep vein thrombosis. The pathological pathway of deep venous obstruction is not completely understood, but is possibly related to these altered haemodynamics. Nowadays, the first two causes of Virchow's triad are encountered in the standardised treatment of an acute DVT or chronic post thrombotic symptoms. Accordingly, the conventional treatment of DVO consists of elastic compressions stockings, exercise, lymph drainage therapy and the use of (pain) medication. ${ }^{12}$ For most patients, the physician selects one treatment or a combination of treatment modalities in an attempt to reduce the symptoms. ${ }^{13}$ However, the venous stasis component is not taken into account because until a few years ago no adequate therapy existed. The introduction of percutaneous angioplasty (PTA) and dedicated venous stents gave opportunities to treat this component. This procedure is already performed in various clinics around the world with good results, both on an individual basis as in case series. ${ }^{14-19}$ Although no comparative studies have been performed, guidelines recommend that PTA as a single treatment should not be offered to patients with deep venous obstructions but a combination with stent placement can be considered and may result in resolution of symptoms. ${ }^{20,21}$

Since patients with established DVO can experience a significant impact on the Quality of life ( $\mathrm{QoL}$ ) which is comparable to chronic pulmonary disease or angina pectoris this is an important issue to focus on. ${ }^{22,23}$ In the past, the effect of deep venous treatment on QoL has been investigated in small series. Furthermore, previous research has mainly focused on patency rates and complication outcomes after deep venous stenting. ${ }^{16}$ Unfortunately, the perceived QoL after interventional treatment has never been compared to this perception after conventional treatment. Currently, there is a debate about the true additional QoL value of this interventional treatment. The aim of this study is to analyze the perceived SF-36 and the VEINES-QoL/Sym QoL in patients treated with stenting compared to conventionally treated patients.

\section{Methods and analysis}

\section{Study design}

This prospective randomized controlled, singe-blind study will be performed in the Maastricht University Medical Care Centre (MUMC). This hospital is a tertiary referral care center for deep venous pathology situated in the Netherlands. Patients with PTS or MTS who are referred to the department of venous surgery at the MUMC will be recruited. 


\section{Study population}

The study population includes all patients with PTS or MTS, called DVO, eligible for interventional percutaneous treatment, who did not undergo previous deep venous intervention that visit the venous surgery department at the MUMC and are willing to participate. All patients have received conservative management for 1 year. Patients with a chronic non-ambulatory status are generally not eligible for interventional treatment. Before admission all patients will be screened for undersigned in- and exclusion criteria. Whenever these cannot be met, patients cannot be considered in this study.

\section{Inclusion criteria}

In order to be eligible to participate in this study, a subject must meet all of the following criteria:

- $\quad$ Age $>18$ years

- Meet criteria for

- PTS (debilitating clinical symptoms) with iliofemoral obstruction on radiological work up expected to be treated solely percutaneous (without endophlebectomy and AV-fistula) based on post thrombotic changes till $1 \mathrm{~cm}$ above the femoral/profundal confluence

OR

- MTS on additional imaging (DUS/MRV/CTV) with clinical symptoms

- Life expectancy of more than one year

- Deep venous thrombosis $>1$ year

- $\quad$ Signed informed consent 


\section{Exclusion criteria}

A potential subject who meets any of the following criteria will be excluded from participation in this study:

- Previous intervention of central veins ( inferior vena cava, iliac veins, common femoral vein) on the affected limb

- Known pregnancy

- Inability to answer Dutch QoL questionnaires or limited communication in Dutch ( spoken and written)

- Contra-indication for prolonged anticoagulant therapy

- $\quad$ Recent, <1 year, DVT or pulmonary embolism

- Known contrast allergy

- Known dialysis or renal insufficiency needing additional preparation for injection of contrast

- $\quad$ Uncontrolled or active coagulopathy or known not correctable bleeding diathesis

- Hypersensitivity to nitinol or nickel

- Known to be, or suspected to be unable to comply with the study protocol (e.a. no permanent address, known to be non-compliant or presenting an unstable psychiatric history)

- Legal incapacity and/or other circumstances rendering the subject unable to understand the nature, scope and possible impact of the study

- Subjects in custody by juridical order

- Subjects who do not agree to the transmission of their pseudonymous data within the liability of documentation and notification

- Close affiliation with the investigational site: e.g. close relative of the investigator or a possibly dependent person (e.g. employee or student of investigational site)

\section{Interventions}

After inclusion, patients will be randomized between conventional therapy and interventional treatment. Randomisation will be stratified for the PTS or MTS group. Patients in the intervention group will be scheduled and treated with regular interventional deep venous stenting. For this interventional treatment, patients should be administered to the patient ward for at least 24 hours. The creatinine, haemoglobin and INR levels will be checked before start of the procedure. INR levels above 4 should be treated with lowering the amount of used anticoagulant tablets and can result in postponement of the procedure until an INR level of $<2.5$ has been reached.

The procedure will be performed in an angiosuite after cleansing and sterile draping of the abdomen and leg. Patients with MTS will be treated with local anaesthesia 
(lidocaine, 1\%) and patients with PTS will receive sedation. All patients will receive an intravenous infusion and the sedated patients will receive a urinary tract catheter. Percutaneous venous access will be performed by sonographic guided puncture of the popliteal, femoral, common femoral or jugular vein . After puncture of the vein, a sheet is introduced with radiologic and contrast agent assistance. Secondly, a guide wire is passed along the affected segment and this segment is dilated with a PTA balloon (with $2 \mathrm{~mm}$ overdilation). During the intervention a bolus of $5000 \mathrm{IE}$ of Heparin will be administered. Afterwards, one or multiple dedicated venous stents (Optimed, GmbH, Germany) will be deployed in the vein and post dilatation with a PTA balloon will follow to optimize the geometry of the stent. Lastly, all patients need to lie down for at least 3 hours to ensure optimal closure of the percutaneous vessel puncture. Pneumatic stockings are used to increase the inflow when immobilized. All patients will receive therapeutic anticoagulation after stenting for a minimum of 6 months. In the bridging period low molecular weight heparins will be used until therapeutic anticoagulation levels are reached. After 6 months, the anticoagulation is continued if the patient was already on anticoagulation before the intervention. Also when stent related issues occur, the anticoagulation might be continued.

Conservative treatment consists of either one or a combination of the following items: pain medication, manual lymphatic drainage therapy, compression stockings and regular post thrombotic anticoagulants therapy. The necessity of each treatment modality, except for therapeutic elastic stockings short (till knee) Class II, will be evaluated on an individual basis in interaction with both the patient as well as the treating physician.

In case of conservative management without any indications for prolonged anticoagulant therapy, this will not be started again.

\section{Outcomes}

\section{Quality of Life}

Quality of life can be measured by numerous questionnaires e.g. with specifically general questionnaires and disease specific questionnaires. The SF-36, is a widelyused questionnaire to measure the general quality of life.

This questionnaire is composed of eight dimensions and mainly focuses on the patients' experiences. A high score in all dimensions reflects a good life quality. ${ }^{24}$

The VEINES-QoL/Sym questionnaire is a 100-point disease-specific scoring questionnaire which can be used to evaluate the psychometric properties of venous disease. This census-paper is a valid and reliable instrument which has been used to evaluate outcomes in previous literature. ${ }^{25,26}$ 
Both, the SF-36 and the VEINES-QoL/Sym will be used to evaluate the effect of the treatment.

The primary outcome evaluated in this study, is the change in QoL measured by the VEINESQoL/Sym score in patients with DVO. The scores will be evaluated at baseline and after 12 months of follow up. A comparison will be made between the intervention (stenting) group and the conventional therapy group (individually based management with short, class II elastic compressions stockings, exercise, lymph drainage therapy and the use of (pain) medication).

Secondary outcomes will be QoL change evaluated by the SF-36, EuroQOL-5D, and pain disability index in patients with DVO. Equal to the primary outcome, these scores will be evaluated at baseline and 12 months follow up. A comparison will be made between the intervention group and the conventional therapy group.

\section{Patency}

The stent patency will be evaluated by duplex ultrasound during every follow up visit. Primary patency is defined as flow in the stent lumen without the need for additional interventional procedures due to stenosis or occlusion. Assisted primary patency is defined as flow in the stent lumen after additional stenting or PTA because of a stenosis with related clinical symptoms. Secondary patency is defined as flow in the stent lumen after additional thrombolysis, thrombectomy, creation of an AV fistula (AVF), restenting or PTA because of previous stent occlusion.

\section{Clinical outcomes}

Venous claudication will be scored positive whenever patients experience onset or worsening of pain during (mild) exercise, which subsides during rest, especially when sitting or lifting the leg.

The Venous Clinical Severity Score (VCSS) and the Villalta score are clinical scores which have been validated for post thrombotic syndrome. (27) Both scores will be analysed before and after treatment and changes will be compared between the intervention group and conventional therapy group.

Since patients with the MTS may have a higher chance of a DVT, and patients with PTS of a recurrent DVT, due to the outflow obstruction, the DVT recurrences will be registered.

Lastly, it is important to analyze the burden of working loss, as DVO can cause invalidating symptoms in daily practice. For this reason, the number of working days loss will be registered for both treatment groups in every follow up visit. Furthermore, modifications in the time and type of work will be evaluated. 
All outcomes will be evaluated at baseline, at 6 weeks' follow-up and at 12 months follow-up by an observer who is blinded to treatment assignment. In the intervention group, patency will be assessed at 2 weeks, 6 weeks, 6 months and 12 months. When a stenosis or occlusion of the stent is seen, the date is registered. Further treatment will be offered and dates of additional treatment will be recorded.

\section{Time line}

Like in regular work-up for patients with deep venous pathology, all eligible patients will have imaging of the veins by duplex ultrasound (DUS) and magnetic resonance venography (MRV) or computed tomography venography (CTV). Related to these radiographic images, the extent of the obstruction or occlusion of the veins will be assessed.

Eligible patients will be contacted and offered the opportunity to participate in the study. After gaining informed consent, patients will be randomized to either conservative treatment or stenting with a 1:2 ratio.

At baseline and during all follow up visits, patients will have a full clinical examination to assess the extent of complaints and clinical manifestations of DVO. The severity of complaints is scored using the Venous Clinical Severity Score (VCSS), venous claudication score, highest $\mathrm{C}$ of CEAP and the Villalta scale.

To assess the QoL, the short-form 36v2 (SF-36v2) will be used for generic QoL and the VEINES-QoL/Sym will be used for the disease specific QoL.

In all patients, a baseline as well as 6 weeks and 12 months follow up ethylenediamine tetraacetic acid, serum and citrate blood sample (a total of $20 \mathrm{ml}$ ) will be requested. All of these blood samples will be taken by the diagnostic lab and send to the Biobank in Maastricht. Blood samples will be frozen for 15 years in order to perform possible future examinations.

Questions about having a (paid) job will be asked and registered at baseline and follow up visits. All patients will visit the outpatient clinic at 6 weeks and 12 months after enrolment into the study. The assessment of all clinical scorings and questionnaires will be accompanied by an independent investigator who is blinded for the type of intervention.

Patients in the conservative treatment group will be asked to take off the therapeutic elastic stockings to obtain the blinding.

The patients in the intervention group will have additional visits at 2 weeks, 12 weeks and 6 months to assess the patency of the stents by duplex ultrasound. When a stenosis or occlusion of the stent is seen, further treatment will be offered. In case of an occlusion this additional treatment can consist of either ultra-sound enhanced catheter-directed thrombolysis or thrombectomy with or without endoplebectomy and the creation of an AV-fistula. In case of a significant stenosis, a PTA with or 
without restenting can be performed. Lastly, in both cases a conservative treatment will be discussed whenever clinical complaints are absent or mild.

After 12 months, all patients in the intervention group will receive a CTV to evaluate the stent patency and stent position.

\begin{tabular}{|c|c|c|c|c|c|c|c|c|}
\hline Stent group & Baseline & Admission & Discharge & $\begin{array}{c}2 \pm 1 \\
\text { week }\end{array}$ & $\begin{array}{c}6 \pm 2 \\
\text { week }\end{array}$ & $\begin{array}{l}12 \pm 4 \\
\text { week }\end{array}$ & $\begin{array}{c}6 \pm 1 \\
\text { month }\end{array}$ & $\begin{array}{c}12 \pm 2 \\
\text { month }\end{array}$ \\
\hline Medical history & $x$ & $x$ & & & & & & \\
\hline CEAP, VCSS, Villalta, VC & $x$ & & & $x$ & $x$ & $x$ & $x$ & $x$ \\
\hline QoL & $x$ & & & $\mathrm{x}$ & $\mathrm{x}$ & $x$ & $x$ & $\mathrm{x}$ \\
\hline Days off work & $x$ & & & $x$ & $x$ & $x$ & $x$ & $x$ \\
\hline Anticoagulation & $x$ & $x$ & $x$ & $x$ & $x$ & $x$ & $x$ & $x$ \\
\hline Adverse events & & & & $x$ & $x$ & $x$ & $x$ & $x$ \\
\hline Laboratory & $\mathrm{x}$ & On indication & $x$ & & $\mathrm{x}$ & & & $x$ \\
\hline Duplex US & & & $\mathrm{x}$ & $\mathrm{x}$ & $x$ & $x$ & $x$ & $x$ \\
\hline \multicolumn{9}{|l|}{ MRV } \\
\hline CTV & & & & & & & & $x$ \\
\hline Conservative group & Baseline & $\begin{array}{c}2 \pm 1 \\
\text { week }\end{array}$ & $\begin{array}{c}6 \pm 2 \\
\text { week }\end{array}$ & \multicolumn{2}{|c|}{$12 \pm 4$ week } & $\begin{array}{c}6 \pm 1 \\
\text { month }\end{array}$ & \multicolumn{2}{|c|}{$12 \pm 2$ month } \\
\hline Medical history & $x$ & - & & \multicolumn{2}{|c|}{-} & \multicolumn{2}{|l|}{ - } & \\
\hline CEAP, VCSS, Villalta, VC & $x$ & - & - & \multicolumn{2}{|c|}{-} & \multicolumn{2}{|l|}{-} & $x$ \\
\hline QoL & $x$ & - & $x$ & \multicolumn{2}{|c|}{-} & \multicolumn{2}{|l|}{-} & $x$ \\
\hline Days off work & $x$ & - & $\mathrm{x}$ & \multicolumn{2}{|c|}{ - } & \multicolumn{2}{|l|}{-} & $x$ \\
\hline Anticoagulation & $x$ & - & $x$ & \multicolumn{2}{|c|}{-} & \multicolumn{2}{|l|}{-} & $x$ \\
\hline Adverse event & & - & $\mathrm{x}$ & \multicolumn{2}{|c|}{-} & \multicolumn{2}{|l|}{-} & $x$ \\
\hline Laboratory & $x$ & - & $x$ & \multicolumn{2}{|c|}{-} & \multicolumn{2}{|l|}{-} & $x$ \\
\hline Duplex US & & - & & \multicolumn{2}{|c|}{-} & \multicolumn{3}{|l|}{-} \\
\hline MRV & & - & & \multicolumn{2}{|c|}{-} & \multicolumn{3}{|l|}{-} \\
\hline CTV & & - & & \multicolumn{2}{|c|}{ - } & \multicolumn{3}{|l|}{-} \\
\hline
\end{tabular}

\section{Withdrawal of individual subjects}

Subjects can leave the study at any time for any reason if they wish to do so. This will not have any consequences for further treatment. The investigator can decide to withdraw a subject from the study for urgent medical reasons. In patients who withdraw from the trial, attempts will be made to retrieve data on the primary and secondary outcomes.

\section{Recruitment}

All patients with PTS or MTS who are referred to the department of venous surgery at the MUMC and meet the inclusion criteria will be asked to participate.

The treating physician will inform potential candidates about this study during regular outpatient visits and will provide them with written study information. They will explicitly ask for the patient's permission to pass the patients name, birth date, 
telephone number and address to the research physician. When interested, the patient is contacted and included by the research physician after 7-14 days. All questions will be answered and written informed consent will be obtained. A separate written consent for blood sample storage will be obtained. All participants will be provided with the contact information of the research physician, to enable contact if any study-related problems are encountered.

\section{Assignment of intervention}

The randomization between interventional and conservative management will be performed with random permuted blocks of 3-6 in a 2:1 ratio (2 stented versus 1 conservative). Randomisation will be stratified for MTS or PTS.

A web based randomization programme will be used for randomization (Alea, Release: 2.2 build: 2070 | amc/ALEA). The randomization will be performed by the research physician after obtaining the patient's consent.

Because the treatment involves an invasive therapy, a sham operation would expose participants to unnecessary risks, and therefore the treatment allocation cannot be blinded. The independent researcher who performs the scoring at follow up moments, will be blinded for treatment allocation.

\section{Data collection, management, and analysis}

In accordance with section 10, subsection 4, of the WMO, the sponsor (MUMC) will suspend the study if there is sufficient ground to assume that continuation of the study will jeopardise the subject's health or safety. The sponsor will notify the accredited METC without undue delay of a temporary halt including the reason for such an action. Subsequently, the study will be suspended pending a further positive decision by the accredited METC. The research physician will take care of informing the included subjects.

All adverse events related to the medical device and adverse events, reported spontaneously by the subject or observed by the research physician or his staff, will be recorded.

Serious adverse events (life-threatening or events leading to permanent impairment as well as in-patient hospitalization or prolongation of existing hospitalization, or medical or surgical intervention to prevent life threatening illness) will be brought under the attention of the coordinating research physician and the head of the department. The research physician will report all SAEs to the sponsor without undue delay, but no later than 72 hours, after obtaining knowledge of the events. The following SAE is excluded for this reporting: persistent or significant disability or incapacity due to post thrombotic syndrome when stents occlude or show a stenosis since this is merely a risk of the treatment. 


\section{Follow-up of adverse events}

All (S)AEs will be followed until they have abated, or until a stable situation has been reached. Depending on the event, the follow up may require additional tests or medical procedures, and/or referral to a general physician or a medical specialist.

\section{Handling and storage of data and documents}

All study documents will be stored in locked cabinets in the University of Maastricht for a period of 15 years. Patients will have a unique trial number not related to their names or birth dates. Only the investigators will have an overview of the trial numbers and patient names. Digital data will be stored at a secure drive in the MUMC. All clinical patient data, which is noted as usual in the electronic patient files, can only be viewed by authorized personnel of the MUMC.

The handling of personal data complies with the Dutch Personal Data Protection Act. All blood samples will be stored in the freezer of the Biobank Maastricht in a coded version. This code will be exactly the same as the code for other patient data. Only the investigators will have an overview of trial numbers and patient names. Blood samples will be frozen for 15 years in order to make future examinations in line with this research possible.

\section{Monitoring and Quality Assurance}

Data monitoring will be performed by members of the Clinical Trial Centre Maastricht (CTCM). The CTCM will monitor the course of the trial and provide ongoing oversight of the data entry. The CTCM is independent from the sponsor and does not recall any competing interests.

At 6 months after study initiation, all bleeding complications will be gathered and reviewed

by an independent Data Safety Monitoring Board with the authority to interrupt the study prematurely, based on significant enhanced bleeding risk or excess morbidity/ mortality in the intervention arm of the study population. Following the initial gathering, the safety monitoring board will review all incidences of bleeding on a regular base (at least once every 6 months). No interim analysis will be performed.

\section{Amendments}

Amendments are changes made to the research after a favourable opinion by the accredited METC has been given. All amendments will be notified to the METC that gave a favourable opinion. Whenever these amendments are substantial, trial participants and trial registries will be informed. 


\section{Temporary halt and (prematurely) end of study report}

The sponsor (MUMC) will notify the accredited METC and the competent authority about the end of the study within a period of 8 weeks. The end of the study is defined as the last visit of the last patient.

The sponsor will notify the METC immediately in case of a temporary halt of the study, including the reason of such an action.

Whenever the study ends prematurely, the sponsor will notify the accredited METC and the competent authority within 15 days, including the reasons for the premature termination.

Within one year after the end of the study, the investigator/sponsor will submit a final study report with the results of the study, including any publications/abstracts of the study, to the accredited METC and the Competent Authority.

\section{Statistical analysis and power calculation}

The data will be analyzed by an intention to treat analysis and per protocol analysis. Efforts will be made to minimize the number of missing values. In patients who withdraw from the trial, attempts will be made to retrieve data on at least the primary outcome by asking the subject to fill out the 12 month questionnaires.

\section{Primary study parameter(s)}

The primary endpoint is the change in quality of life at 12 months (on the disease specific Veines Qol/Sym questionnaire) from baseline. Change in Qol from baseline between the randomized groups will be compared and tested for statistical significance using analysis of covariance (ANCOVA). The dependent variable will be the QoL end-score and baseline Qol and group will be entered as covariates.

For evaluation of patency of stents a Kaplan-Meier survival analysis will be used.

The comparability of baseline characteristics between groups will be evaluated. Nominal and categorical data will be presented as absolute numbers and percentages. Continuous data will be presented as mean values with standard deviation or as median values with interquartile range (IQR), depending on normality of distribution. In case of imbalances, linear multivariate regression analysis will be used to adjust for baseline differences. For all analysis, a $\mathrm{p}$-value $\leq .05$ is being used to indicate statistical significance.

\section{Power calculation}

The sample size calculation is based on the consideration that an improvement in Qol (from baseline to 12 months) of 14 points on the disease specific Veines Qol/Sym questionnaire can be considered as minimally clinical relevant. To detect at least 14 points improvement $(\mathrm{sd}=22)$ with a power of $90 \%$ and 2 -sided alpha=5\% and 
randomization ratio of 2:1 (intervention: conservative), A total of 117 patients is required (78 in the intervention group vs 39 in the conservatively treated group). To account for loss to follow-up of $\pm 10 \%$ of patients, a total of 130 patients need to be included ( 86 in the intervention group vs. 44 in the conservatively treated group. We will perform a subgroup analysis (PTS vs. MTS) to evaluate consistency of the effect of intervention across subgroups.

\section{Discussion}

Previous systematic reviews have shown low complication rates $(0-8.7 \%)$ and high technical successes (up to 98\%) for deep venous stenting. ${ }^{14,16}$ Reviews also show a relief of oedema and pain in up to $64-68 \%$ and respectively $82 \%$ of patients. Furthermore, primary patency rates between $32 \%$ and $98.7 \%$ and secondary patency rates of $66 \%$ to $96 \%$ are reported. ${ }^{14}$ Although these reviews show favourable results, the outcomes are mainly based on retrospective, single centre, cohort trials. Besides this, the main important outcome for patients, that is the QoL they experience in relation to their complaints, is not continuously examined.

Therefore, this research emphasizes on the reported QoL of PTS and MTS patients. Moreover, the prospectively randomized design underlines the strength of this study. The outcomes of this study will clarify if the QoL in DVO will improve and to which extent it will change. Furthermore, this study will show if the QoL outcomes are comparable for both interventional treatment and conservative management. Additionally, it is possible to differentiate between outcomes in PTS and MTS. Since PTS and MTS are different entities, this study will emphasize on the effect in both patient groups and show the difference in QoL. We hypothesize that the change in QoL in MTS patients will be different from PTS patients since PTS causes deep venous obstruction as well as venous insufficiency. With deep venous stenting, complaints related to valve reflux will not be treated and thus not all complaints will resolve.

\section{Limitations}

Since this is a randomized trial, it is likely that there will be systematic difference between the patients who are willing to participate in this trial compared to those who are not. This can eventually lead to an outcome that cannot be translated to all patients with DVO. In contrary, the patients who are not willing to participate in this study, may not experience the symptoms in a way that their daily activities are interfered and do not opt for interventional treatment at all. 
Secondly, the MUMC is the most experienced centre in deep venous stenting in the Netherlands. The investigated interventional therapy requires in-depth knowledge and related clinical skills. This may interfere the replication of the interventional treatment and specifically, the related outcomes, across other institutions.

If a similar RCT would be performed in other countries, it would be beneficial to pool all data and perform a meta-analysis. However, the treatment diagnostics and definition of a MTS should be standardized to compare actual outcomes. Especially the definition of MTS may alter the decision to perform a deep venous intervention. ${ }^{28}$ In our centre, treatment is based on a combination of DUS, MRV and phlebography showing a compression of $>50 \%$ and apparent collaterals. Other countries may use an intravascular ultrasound (IVUS) to plan their treatment decisions. The pooling of these data in a meta-analysis may be a challenge. 


\section{References}

1. Meissner MH, Eklof B, Smith PC, Dalsing MC, DePalma RG, Gloviczki P, et al. Secondary chronic venous disorders. J Vasc Surg. 2007;46 Suppl S:68s-83s.

2. Nordstrom M, Lindblad B, Bergqvist D, Kjellstrom T. A prospective study of the incidence of deep-vein thrombosis within a defined urban population. J Intern Med. 1992;232(2):155-60.

3. Johansson $M$, Johansson $L$, Lind $M$. Incidence of venous thromboembolism in northern Sweden (VEINS): a population-based study. Thromb J. 2014;12(1):6.

4. Cogo A, Lensing AW, Prandoni P, Hirsh J. Distribution of thrombosis in patients with symptomatic deep vein thrombosis. Implications for simplifying the diagnostic process with compression ultrasound. Arch Intern Med. 1993;153(24):2777-80.

5. Jezovnik $M K$, Poredos $P$. Factors influencing the recanalisation rate of deep venous thrombosis. Int Angiol. 2012;31(2):169-75.

6. Akesson H, Brudin L, Dahlstrom JA, Eklof B, Ohlin P, Plate G. Venous function assessed during a 5 year period after acute ilio-femoral venous thrombosis treated with anticoagulation. Eur J Vasc Surg. 1990;4(1):43-8.

7. Plate G, Eklof B, Norgren L, Ohlin P, Dahlstrom JA. Venous thrombectomy for iliofemoral vein thrombosis--10-year results of a prospective randomised study. Eur J Vasc Endovasc Surg. 1997;14(5): 367-74.

8. Kahn SR, Shbaklo H, Lamping DL, Holcroft CA, Shrier I, Miron MJ, et al. Determinants of health-related quality of life during the 2 years following deep vein thrombosis. J Thromb Haemost. 2008;6(7):110512.

9. Rabinovich A, Kahn SR. The postthrombotic syndrome: current evidence and future challenges. J Thromb Haemost. 2016.

10. Lugo-Fagundo C, Nance JW, Johnson PT, Fishman EK. May-Thurner syndrome: MDCT findings and clinical correlates. Abdom Radiol (NY). 2016;41(10):2026-30.

11. May R, Thurner J. [A vascular spur in the vena iliaca communis sinistra as a cause of predominantly left-sided thrombosis of the pelvic veins]. Z Kreislaufforsch. 1956;45(23-24):912-22.

12. Kearon C, Akl EA, Ornelas J, Blaivas A, Jimenez D, Bounameaux H, et al. Antithrombotic Therapy for VTE Disease: CHEST Guideline and Expert Panel Report. Chest. 2016;149(2):315-52.

13. Henke PK, Comerota AJ. An update on etiology, prevention, and therapy of postthrombotic syndrome. J Vasc Surg. 2011;53(2):500-9.

14. Seager MJ, Busuttil A, Dharmarajah B, Davies AH. Editor's Choice-- A Systematic Review of Endovenous Stenting in Chronic Venous Disease Secondary to Iliac Vein Obstruction. Eur J Vasc Endovasc Surg. 2016;51(1):100-20.

15. Falcoz MT, Falvo N, Aho-Glele S, Demaistre E, Galland C, Favelier S, et al. Endovascular stent placement for chronic post-thrombotic symptomatic ilio-femoral venous obstructive lesions: a singlecenter study of safety, efficacy and quality-of-life improvement. Quantitative imaging in medicine and surgery. 2016;6(4):342-52.

16. Razavi MK, Jaff MR, Miller LE. Safety and Effectiveness of Stent Placement for lliofemoral Venous Outflow Obstruction: Systematic Review and Meta-Analysis. Circ Cardiovasc Interv. 2015;8(10): e002772.

17. Wen-da W, Yu Z, Yue-Xin C. Stenting for chronic obstructive venous disease: A current comprehensive meta-analysis and systematic review. Phlebology. 2016;31(6):376-89.

18. Raju S. Treatment of iliac-caval outflow obstruction. Semin Vasc Surg. 2015;28(1):47-53.

19. Neglen P, Hollis KC, Olivier J, Raju S. Stenting of the venous outflow in chronic venous disease: longterm stent-related outcome, clinical, and hemodynamic result. J Vasc Surg. 2007;46(5):979-90.

20. Wittens C, Davies AH, Baekgaard N, Broholm R, Cavezzi A, Chastanet S, et al. Editor's Choice Management of Chronic Venous Disease: Clinical Practice Guidelines of the European Society for Vascular Surgery (ESVS). Eur J Vasc Endovasc Surg. 2015;49(6):678-737.

21. Mahnken AH, Thomson K, de Haan M, O'Sullivan GJ. CIRSE standards of practice guidelines on iliocaval stenting. Cardiovasc Interv Radiol. 2014;37(4):889-97. 
22. Kahn SR, Ducruet T, Lamping DL, Arsenault L, Miron MJ, Roussin A, et al. Prospective evaluation of health-related quality of life in patients with deep venous thrombosis. Arch Intern Med. 2005;165(10): 1173-8.

23. Lubberts B, Paulino Pereira NR, Kabrhel C, Kuter DJ, DiGiovanni CW. What is the effect of venous thromboembolism and related complications on patient reported health-related quality of life? A meta-analysis. Thromb Haemost. 2016;116(3):417-31.

24. Jenkinson C, Stewart-Brown S, Petersen S, Paice C. Assessment of the SF-36 version 2 in the United Kingdom. J Epidemiol Community Health. 1999;53(1):46-50.

25. Mean M, Limacher A, Kahn SR, Aujesky D. The VEINES-QOL/Sym questionnaire is a reliable and valid disease-specific quality of life measure for deep vein thrombosis in elderly patients. Qual Life Res. 2014;23(9):2463-71.

26. Kahn SR, Lamping DL, Ducruet T, Arsenault L, Miron MJ, Roussin A, et al. VEINES-QOL/Sym questionnaire was a reliable and valid disease-specific quality of life measure for deep venous thrombosis. J Clin Epidemiol. 2006;59(10):1049-56.

27. Lattimer CR, Kalodiki E, Azzam M, Geroulakos G. Validation of the Villalta scale in assessing postthrombotic syndrome using clinical, duplex, and hemodynamic comparators. J Vasc Surg Venous Lymphat Disord. 2014;2(1):8-14.

28. Hameed M, Onida S, Davies AH. What is pathological May-Thurner syndrome? Phlebology. 2017;32(7):440-2. 



\section{Chapter 11}

General discussion and summary 



\section{General discussion and summary}

Deep venous obstruction is a common condition that has gained increasing attention for its high socioeconomic burden and negative effect on a patients' quality of life (QOL). ${ }^{1-3}$ The obstruction is either a result of chronic trabeculation after a previous deep venous thrombosis (DVT) or extrinsic compression of which the iliac vein compression syndrome (IVCS) is most prevalent. ${ }^{4}$

For years, the primary treatment for DVT focused on initial symptoms and the prevention of long-term complications. As a result, there were only conservative treatment options. In the 1930s, Jobsts noticed fewer detectable symptoms in immobilized patients and subsequently invented the first compression stockings. ${ }^{5}$ In the 1940's, the number of chronic venous insufficiencies dramatically dropped due to the introduction of therapy with anticoagulants. ${ }^{6}$

However, research showed that with conservative treatment, only part of the iliac DVTs dissolved completely. ${ }^{2,7,8}$ The remaining cases experienced post-thrombotic obstruction as a result of inadequate recanalization and valve incompetence, which in turn is caused by the destruction of the valve-closing mechanism. ${ }^{9,10}$ Consequently, patients developed complaints such as pain during ambulation, and cramps or heaviness of the leg, known as venous claudication, related to venous hypertension. $^{11,12}$

Consequently, interventional techniques were rapidly developed to address the need for improvement in primary and secondary therapy. In recent decades, advances in endovascular treatment and imaging techniques have revolutionized the treatment of patients with deep venous obstructions. Before the 1990s, open bypass surgery was performed, but exclusively in cases with severe deep venous pathology. These major surgical interventions did not prove to be a sustainable solution due to the weak to moderate patency rates and moderate clinical outcomes. ${ }^{13,14}$ The following years saw the creation of dedicated venous imaging protocols, improvements in angiographic techniques, and, at a later stage, the introduction of intravascular ultrasound (IVUS). As a result, minimally invasive percutaneous transluminal angioplasty (PTA) and stenting were introduced to substitute the open surgical reconstructions. ${ }^{15,16}$ This led to an overall improvement in the treatment of patients with deep venous obstructions. The endovascular interventions performed in several institutes around the world led to several small case series with good clinical results.

Despite these early successes, it became apparent that venous interventions require different approaches when compared with the mainstream of endovascular arterial interventions. First, veins have thinner media than arteries, and they possess valves that allow blood to flow against gravity. Other components that allow this flow include muscular compression and negative intrathoracic pressure. Next, the thinner media allows for greater distensibility. Specifically, due to anatomical differences, low 
venous pressure and a different underlying pathology, dedicated teams developed in time to evaluate pathology and optimal treatment plans.

In recent decades, several imaging modalities have been used to diagnose and understand venous pathology. With increasing knowledge, it was found that the complete vein system should be analyzed to identify the cause of venous problems. Evaluation was required of not only the leg veins, but also the abdominal and pelvic veins in order to understand the often impaired function of femoral or popliteal vein segments. Pre-interventional evaluation of deep venous pathology by duplex ultrasound (DUS) is one non-invasive option that gives both anatomical and hemodynamical information of the venous system. However, DUS is impaired by operator dependent factors, which require extensive experience, and patient dependent factors such as obesity, uncompressible air in the bowel, ulceration or thickened skin. This led to the development of venous cross-sectional-imaging modalities such as CTV and MRV. ${ }^{17,18}$ CTV can recognize vein obstruction or compression and helps with the recognition of typical collateralization patterns. It is widely available around the world; however, it uses ionizing radiation. Another disadvantage relies in the fact that sufficient and homogeneous opacification remains a challenge. In the 1990s, MRV was used to evaluate venous pathology with the main advantage of visualization of detailed vein wall changes such as webs, spurs and trabeculae. Besides, it does not use radiation and there is no use for iodine contrast. The disadvantages of MRV include non-availability of MR scanners due to its high costs, in several parts around the world and contraindications in patients with claustrophobia or patients with metallic fragments. As an overall result, a combination of imaging modalities should be used to provide as much as information as possible before treatment options can be discussed.

With increasing knowledge and improvements in treatment plans for patients with deep venous pathology, a dedicated venous team was created in the Netherlands in 2012, and deep venous interventions were introduced. Our knowledge regarding this subject, originating from our six-year endovascular treatment experience, has been described in Chapter 2. Based on the underlying pathology and extent of postthrombotic changes detected on dedicated MRV scans and perioperative phlebographies three groups of pathologies were treated. The first group consisted of patients who received percutaneous stenting to treat non-thrombotic iliac vein compression syndrome (IVCS group). The second group consisted of patients with post-thrombotic syndrome (PTS) who were treated by percutaneous stent placement (P-PTS group) and the last group consisted of PTS patients with obstruction involving the veins peripherally to the saphenofemoral junction (SFJ). These patients underwent a hybrid procedure, combining stenting with open surgical desobliteration (H-PTS group). 
Since the primary goal of stent placement is the reduction of clinical complaints, clinical scores were evaluated after 24 months. Results showed an improvement in Villalta scores, specifically in post-thrombotic patients. Moreover, of the 369 treated patients, venous claudication subsided in $90 \%$ of the IVCS group, $82 \%$ of the P-PTS group and $83 \%$ of the H-PTS group. Although former reviews have not been able to pool clinical data in a meta-analysis, some studies have indicated an improvement in clinical outcomes equal to the results presented in our study. For post-thrombotic subjects at the 21 months follow-up, Blanch Alerany et al. have previously shown a significant improvement in both the VCSS from 8 to 2 and the Villalta scale from 15 to $2(P<0.001) .^{19}$

In addition to the clinical outcomes, technical outcomes and related patency scores were analyzed. In our study, this resulted in a primary patency, - assisted primary patency - and secondary patency rate of $90 \%, 100 \%$ and $100 \%$ for the IVCS group and $64 \%, 81 \%$, and $89 \%$, respectively, for the P-PTS group at the 60 month follow-up. In contrast, the H-PTS group, showed patency rates of $37 \%, 62 \%$ and $72 \%$, respectively, at the 36 month follow-up. Interestingly, although different diagnostics, interventional techniques, and stent types were used, the outcomes of percutaneous treated patients were comparable to earlier studies, with primary patency rates between $32 \%$ and $98.7 \%$ and secondary patency rates of $66 \%$ to $96 \%{ }^{20-22}$

In early treatments, stents specifically developed for the arterial system were used. These stents were suboptimal, because the pathology in the venous system exists predominantly due to intraluminal vein scarification and extra luminal compression, distinct from the athero-thrombotic events in arteries. ${ }^{23-25}$ In the venous system it is important to have sufficient crush resistance and outward radial force to withstand external compression. Additionally, anatomical locations with repeatable flexion stress, such as the internal/external iliac vein confluence and at the level of the inguinal ligament, require stents compatible to this range of motion. The stents used in early treatments are less able to allow for adequate radial force, showed in-stent restenosis at vulnerable anatomical locations and are more prone to deployment complications. The often-used balloon expendable Wallstent is comprised of braided Elgiloy. This stent type shows foreshortening of the stent ends during balloon expansion and is specifically difficult to deploy at the caval-iliac confluence. The stent shows sufficient radial force when stent ends are fixed, which may not be the case at the caval-iliac confluence because stents are inserted above the confluence without compromising inflow of the contralateral leg. ${ }^{25-27}$

Because of the shortcomings of early stents, dedicated, self-expandable venous stents were later developed and introduced, with favourable properties such as a higher flexibility and higher radial forces. These stents allow physicians to treat long venous segments. Additionally, these stents are made of nitinol with thermal shape memory and super elasticity which accounts for recovery of the stent shape when external 
load is lowered. ${ }^{26}$ In contrast, balloon expandable stents will irreversibly be deformed if external load exceeds the hoop strength. ${ }^{28}$

Shape recovery of dedicated venous stents is thought to reduce in stent-related complications, such as kinking, fracturing, and tapering. Furthermore, the open-cell design in the dedicated venous stents could result in lower contra lateral DVT incidences. ${ }^{29}$ This result is theorized because the smaller interstices of the more rigid, closed cell stents appear to become lined with neointima more often than those dedicated venous stents, which could allow for less flow and as a result jeopardize the contralateral flow.

Currently, studies comparing the clinical results and patency outcomes of these dedicated venous stents with the more rigid stents are lacking. Nevertheless, favourable results have been described in case series treated with dedicated venous stents. ${ }^{26,30}$ Stuck et al. presented an $85 \%$ primary patency in post-thrombotic and IVCS patients treated with a Sinus Obliquus stent. ${ }^{30}$ Subsequently, a decrease of 6 points and 3 points on the Villalta scale and the VCSS, respectively, were found at the 10 month follow-up. In Chapter 3, we present our investigation of outcomes when a dedicated Sinus Venous stent was used. In a group of 200 patients, the mean Villalta scale decreased from 10.5 to 5.3 at the latest follow-up $(P<0.001)$ and the VCSS score decreased by a total of 3 points $(P<0.001)$. Overall patency scores revealed a primary patency of $68 \%$, an assisted primary patency of $83 \%$, and a secondary patency of $90 \%$ with a median follow-up of 12 (11-12) months.

As clinical scores and short-term patency outcomes seemed comparable for both the dedicated venous stents and the more rigid stents, our attention shifted to the number of related complications. Although previous studies are largely retrospective, single-center cohort trials, they have shown high technical success up to $98 \%$, and low complication rates $(0-8.7 \%)$ in endovenous stenting for chronic venous obstructive disease. ${ }^{20-22}$ By comparison, the results of Chapter 3 of this thesis show, 122 (61\%) patients with no complication during the follow-up period. The most frequent complications seen were in-stent restenosis $(n=23)$ or occlusion $(n=24)$ of the stented tract. Furthermore, we did not find stent-related issues such as kinking, fracturing, or angulation. We did observe that the hybrid procedures displayed the highest complication numbers, adverting to the more extensive pathology and related intervention.

In contrast to published reviews, this thesis presents a large group of hybrid interventions and shows the highest rate of post-interventional complication as $81 \%$ and the highest rate of re-interventions as $59 \%$. These findings are consistent with our expectations, since findings from previous literature have shown that reduced patency and subsequently less favourable clinical outcomes are apparent whenever inflow is inadequate. ${ }^{31-33}$ As a consequence, stent deployment peripherally to the sapheno-femoral junction (SFJ) is not specifically addressed in current guidelines. ${ }^{34}$ 
While treating more patients with deep venous obstructions, it was noticed that a large group of patients with some post-thrombotic changes peripherally to the SFJ had been evaluated at our center. For this reason, we started searching for an interventional therapy to treat patients with post-thrombotic obstructions that also included the vein segments peripherally to the SFJ. The main challenge in doing so was to find patients with a non-fibrotic venous segment into which the stent could be placed. These non-fibrotic venous segments play an indispensable part in giving support to the widely held belief that stenting should be performed "from healthy to healthy". ${ }^{35}$

Because available treatment options were limited, some of our patients had already been treated by both an endophlebectomy of the common femoral vein (CFV), and arterio venous fistula (AVF), that is, by hybrid intervention. This was especially the case for patients with post-thrombotic changes extending into the CFV or for patients in which it was expected that stenting of the CFV would displace intraluminal tissue across the orifices of the femoral vein (FV) and deep femoral vein (DFV). After stent deployment, an AVF was created to generate initial high flow and confine thrombus formation in the recently desobstructed area.

As we noticed that these hybrid procedures were more prone to complications, specifically to stenosis peripherally to the CFV, the geometry of the fistulas was changed. ${ }^{36}$ Initially, it was thought that good inflow in the complete recanalized and operated tract was of main importance; AVFs were thus placed in a straight-shape and caudally from the endophlebectomy area. In time, this resulted in functional lumen obstruction and patency loss due to intimal hyperplasia. Therefore, the geometry of AVFs was changed to a loop-like more cranial position where lumen diameter is larger and therefore should reduce areas of shear stress and diminish functional lumen obstruction. $^{37}$

In evaluating the outcomes of the two geometry techniques, a distinction was made between the two groups, comparing cranially placed fistulas with caudally fistulas. Unfortunately, no statistical significant difference in outcome could be found; however, the risk of occlusion and stent-related complications tended to be in favour of cranially placed fistulas. Consequently, fistulas were created in a loop like fashion and follow up was conducted to evaluate possible short term and mid-term complications. The development of potential peripherally stenosis remained a multifactorial on-going process in which vein wall injury and AVF flow likely played an important role. In addition, the long-term changes caused by an AVF were unknown and might have included on-going intimal hyperplasia ${ }^{37}$ or cardiac overload. In order to limit these complications, a solution was searched and implemented in standard care: As it was theorized that endothelial covering of the stent was present in $86 \%$ of stent struts after 56 days, we evaluated the venous flow 6 weeks to 3 months after the initial procedure to decide whether the AVF should remain patent or whether it was possible to close the AVF. All patients had a scheduled endovascular occlusion of 
the AVF. The contralateral artery was punctured percutaneously and a temporary balloon occlusion of the AVF was performed. Next, a phlebography was performed with contrast administration from the foot veins in the index leg. The contrast washout was evaluated and thought to be sufficient when complete washout was seen within 4 seconds. With sufficient contrast washout, a definite AVF occlusion with an Amplatzer plug was performed. In patients with insufficient contrast washout we evaluated whether stent related issues were the main cause of inadequate flow.

Since we did not find a loss of patency due to kinking or tapering but did notice peripherally stenosis, we evaluated whether stenting peripherally to the CFV was feasible after a primary hybrid intervention. As described in Chapter 4, 24 (38\%) of 64 patients had additional stenting in the FV or DFV. In all subjects, a temporary balloon occlusion of the AVF was performed to evaluate the venous inflow into the stents. When inflow was deemed insufficient, additional stenting was performed in the vessel with the most dominant inflow, and the AVF closure was postponed. The primary, assisted primary, and secondary patency rates were $60 \%, 70 \%$ and $70 \%$ respectively, and the Villalta score was reduced by 6.2 points $(P<0.001)$, and the VCSS by 2.7 points $(P=0.034)$ at a median follow-up of 14 -months. As a result, we showed that in these selected cases, peripherally stenting is possible as a bailout option with a low complication rate and good clinical outcomes.

With these results, compared with the high number of complication rates and reintervention rates after primary hybrid procedures, it was postulated whether it would be possible to select cases in which peripherally stenting could be performed initially. During the selection of patients, we took into consideration that endophlebectomy might be the best option for patients with extensive postthrombotic pathology in the potential target vessels. For example, for patients with extensive pathology in the FV and DFV and with small lumen diameter, the hybrid procedure seems to be the best option. Moreover, we allowed for higher thrombosis risk due to a relatively low flow state in a long-stented tract.

Chapter 5 describes the results of patients who were treated with primary stenting peripherally to the CFV. A total of 14 subjects were treated with primary, assisted primary and secondary patency rates of $92 \%$ at a median follow-up of 481 days. The VCSS decreased from a mean of 8.9 to a mean of $6.4(P=0.03)$. The Villalta scale decreased with a mean of 11.7 to $4.3(P=0.003)$. Consequently, we believe that stenting through the femoral confluence into a dominant inflow vessel, is a viable alternative for a specific group of patients. These results should not be overstated, however, since no comparative studies have been performed by other centers. Additionally there is the possibility that the results shown in Chapter $\mathbf{5}$ are biased, since we can only review the outcomes after treatment. Therefore, further analyses through randomized trials are required.

Future research should focus on the technical optimization of treating deep venous pathologies peripherally to the SFJ. Less invasive treatments could be useful 
specifically for those cases that require a higher inflow. At this time, it is not known what degree of obstruction restricts venous flow significantly and no tests are available to accurately diagnose a hemodynamically significant obstruction. An obstruction of more than $50 \%$ has been chosen as an arbitrary cutoff because favorable results have been presented after stenting in this group of subjects. Imaging techniques such as dedicated duplex protocols and MRI protocols should allow the measurement of flow rates in a reproducible way. With focus on aforementioned general problems it should be more convenient to compare results in different groups.

Based on the results presented in this thesis, it is likely that inflow plays a superior role in stent patency compared with stent length. Future research could focus on this question in order to optimize treatment strategy; Currently, patients wear compression stocking or intermittent pneumatic compression stockings following intervention. Some theories describe nerve stimulation to create muscle contraction and to maintain a constant flow through the stented tract. Small series in healthy subjects have been described with positive effects in flow generation. ${ }^{38}$ All these ideas should be elaborated and tested in clinical practice to optimize treatment plans.

In addition to the insights gained regarding technical optimization, this thesis points to other factors contributing to treatment outcomes. ${ }^{39}$ In this respect, we searched for different items leading to clinical optimization and patient selection. This process started with the basic principles of a first consultation of patients with their physician. During this consultation, a thorough anamnesis and clinical examination is standard care and can provide massive amounts of knowledge in deep venous pathology. To treat patients with deep venous obstructive disease, physicians should however recognize venous clinical signs and relate them to venous pathology. For instance, with clinical examination it should be recognized whether there is a clinically visible prepubic collateral but also what the meaning of this item could be. Although prepubic collaterals have been previously associated with deep venous obstructions, there was no research that thoroughly examines this possible correlation. ${ }^{40}$ Chapter 6 of this thesis, explores the preclinical course of these deep venous obstructive clinical signs, and more specifically, the presence of prepubic collaterals.

Based on the results of this chapter, a referral for additional imaging is warranted in the case of clinically visible prepubic collaterals because it resulted in a positive predictive value of any obstruction at the level of or above the groin in $93 \%$ of cases. A specificity of $86 \%$ was found, indicating few false negative results; however, sensitivity was $53 \%$ for any obstruction at the level of the groin or higher. These results may be biased because the data were extracted from a population with clinical complaints related to leg problems, venous ulceration, or previous deep vein thrombosis. In the general population, in particular, pathology of the liver or pathology of gynecologic origin cannot be excluded and should also be considered as presented by the low 
sensitivity of the prepubic collateral. ${ }^{41,42}$ However, it is recommended that these collaterals not be removed unless deep venous pathology has been ruled out. It is strongly advised to inquire into deep venous pathology, mainly in symptomatic patients, because of the non-invasiveness of additional imaging. When pathology is found, it should be analyzed whether interventional treatment can be an option.

The viability of interventional treatment is dependent on the extensity of the obstruction. The first step, therefore, is to determine how extensive the obstruction is. To this end, patients should be referred to centers with dedicated deep venous knowledge. Dedicated venous duplex protocols, in-depth knowledge regarding deep venous pathology and dedicated additional diagnostics are indispensable when treating these patients. Generally, the first imaging tool should comprise venous evaluation by duplex ultrasound. ${ }^{43}$ This technique has proven to be adequate for both anatomical and hemodynamical evaluation. ${ }^{44}$ However, there are some disadvantages. For less experienced practitioners, for example, it can be difficult to accurately map the abdominal and pelvic veins. Moreover, there are some patientspecific factors, such as obesity, edema, or bowel gas, which can negatively influence diagnostic accuracy. Additional diagnostics can be used to mitigate these factors: Diagnostic 3D tools include computed tomography venography (CTV) and magnetic resonance venography (MRV), which can provide additional information on deep venous stenosis or obstruction in the abdominal or pelvic veins. ${ }^{18,45,46}$ Since the dedicated MRV protocol was introduced a few years ago, multiple aspects of treatment planning have improved. During the analysis of the MR scans, we noticed that certain specific patterns were apparent in post-thrombotic patients. We found alternative drainage pathways that were a result of recanalization after a DVT. Regarding caval vein obstructions specifically, there are studies describing the azygos and hemiazygos, gonadal, spinal or abdominal veins as predominant collaterals. ${ }^{41,47}$

With respect to endovenous interventional treatment, it is hypothesized that developed collateral veins become redundant when the normal physiological route is restored. Former studies have already shown that occlusions of the inferior caval and iliac veins are the most challenging cases to treat. ${ }^{48}$ However, the hemodynamic importance of collateral pathways and their effects on stent patency after an intervention have not yet been investigated. In an effort to explore various explanations for stent failure, we reanalyzed the collateral veins of treated patients on preoperative MRV scans. Chapter 7 has shown that a vena cava occlusion and the surface area measurements of the azygos and hemiazygos vein were positive and statistically significantly related (OR $1.01,95 \% \mathrm{Cl} 1.003-1.019, P=0.004$ and OR 1.007, $95 \% \mathrm{Cl}$ 1.001-1.013, $P=0.004$, respectively). An azygos surface area measurement of $23(P<0.001)$ and a hemiazygos surface area measurement of $40(P=0.008)$ has been shown as a cut-off point for higher occlusion rates.

Interestingly, it has been observed that collateral patterns can be one of the most prominent signs of significant obstruction not only in intraluminal changes ${ }^{49}$ but also 
in extraluminal vein compression. Subsequently, the presence of collateral veins in angiography has been considered a treatment indication. ${ }^{4}$ One of the more intriguing aspects of vein compression syndromes is the fact that the prevalence of (significant) deep vein compression in the general population is still unknown. ${ }^{50,51}$ Additionally, it is still difficult in practice to predict which patients will benefit from interventional treatment. For these reasons, we studied the radiographic signs of an asymptomatic population; the results of this study is presented in Chapter 8. It is noteworthy that we found a remarkably high percentage of anomalous angiographic examinations of the deep pelvic veins in young and healthy subjects. Chapter 8 describes our discovery of a potential imbalance between clinical signs and imaging findings which can lead to overtreatment. It should be noted that although intravascular ultrasound (IVUS) seems a promising tool in the evaluation of subtle intravascular changes, this type of ultrasound was not used due to its invasiveness. Although the recently published VIDIO trial showed IVUS to be superior to conventional phlebography in diagnosing iliac stenotic lesions, ${ }^{52}$ IVUS remains an expensive diagnostic tool that is not routinely used by interventional physicians throughout the world. Moreover, the question remains whether all lesions found through IVUS are of clinical importance and require interventional therapy. Therefore, further research is necessary to analyze which groups of patients benefit from interventional treatment.

The clinical outcomes and post interventional benefits are currently evaluated by clinical examination and follow up of clinical scores. Although we know stenting is a good option for many patients, some show no clinical improvement or even show a worsening of complaints. These findings have been presented in Chapter 2. Given our current knowledge, it is difficult to predict the patients for whom deep venous treatment will elicit improvement. This can be explained in part by the fact that an abnormal scientific route has been taken in recent years.

Deep venous stenting was started several years ago without specific insight into or knowledge of the exact pathophysiologic hemodynamic mechanism. Earlier studies have shown that angioplasty and stenting are safe and effective, but current evidence is weak and mainly based on single-center retrospective studies. Large clinical data with animal models or pressure measurements are lacking. Currently, the results of treated patients are analyzed and translated back to possible explanations based on a basic understanding of deep venous pathology.

Accurate and consistent diagnosis is an important issue before treatment options are discussed. According to earlier studies, PTS occurs in $20 \%-50 \%$ of patients after an iliofemoral DVT. ${ }^{2,7-9}$ The main reasons for this wide range include the different diagnostic tools that were used to assess PTS in these studies; the time interval between acute DVT and PTS assessment and the number of PTS assessments performed heterogeneously in the literature.

With general clinical signs such as pain and edema, PTS has been shown to be a nonspecific syndrome, and other diseases may present with similar symptoms. In practice, 
a former DVT and leg symptoms are thought to be indicative of PTS and are classified as such. Thus far, there is no single objective test that can diagnose PTS. Clinical scores such as VCSS and Villalta are being used to longitudinally describe clinical symptoms, clinical signs and the effect of deep venous stenting. These tools have however various advantages and disadvantages.

First, the VCSS was initially developed to register chronic venous disease signs and symptoms over time. ${ }^{53}$ The main disadvantage of this scoring item lies in the fact that some patients with normal ambulatory venous pressure have received a diagnosis of severe PTS, and clinical signs are taken into account only in a single scoring item. Moreover, this score is difficult to use longitudinally because skin pathology, as included in current scorings, will not change and are very subjective. In general, scores decrease after interventional treatment, when there is relief of edema or, pain or when patients are able to wear stockings to a lesser extent.

Second, the Villalta scale was developed by Villalta et al. and published as an abstract only. The Villalta scale has been described as having a good correlation with QOL and showing an excellent interobserver reliability. However, some aspects of the scale are not yet validated. This could result in a biased inter-rater reliability. Moreover, the Villalta scale contains 12 total items, comprising 5 items related to symptoms and 7 items related to clinical signs. This scoring system's main disadvantage is some of the items must be scored by both the physician and the patient. As a consequence, patients must visit a physician to receive a total Villalta score. To this end, the patientreported Villalta scale was developed, which was shown in previous studies to have promising results. Unfortunately, it has not been evaluated for use by associations with other PTS markers such as health-related quality of life (HRQOL). ${ }^{54}$

In order to address the need for a way to assess the efficacy of treatment over time and by one person, we developed the Self-Assessed Venous Severity-Questionnaire (SAVS-Q). This questionnaire included a visually guided Villalta scale, VCSS score, QoL scale, and questions regarding venous claudication. In this study, two physicians/ researchers scored all items; subsequently, the patient scored all items independently (Q1 and Q2). A total of 64 patients were eligible for inclusion, and a subset of 39 completed a second questionnaire 1-7 days thereafter. The physician-reported VCSS score showed an interclass correlation of $0.51, P<0.01$ when compared to the patients' self-assessed score. The VCSS reliability was highest in the physician and trained researcher group with an interclass correlation of $0.75, P<0.01$. When the patients' self-assessed Villalta scale was compared to the physician-reported scale a reliability of $\mathrm{K} 0.04$ was found. A comparison of the VEINES/QOL scores and clinical scores showed the highest correlation between Q1 and Q2. The venous claudication questions showed a very high correlation with the VEINES/QOL of $-0.97 P<0.01$. We believe that this SAVS-Q can be used as the basis of future venous questionnaires but there are a few limitations which should be analysed in further detail. Specifically, scores should be reproducible in order for them to be usable for follow up of 
treatment. That is, the subjectivity of scoring items should be minimized by describing fixed items contributing to a particular score. For instance, hyperpigmentation is scored as mild, moderate or severe. If we agree to include this item in the final venous questionnaire, a colour scale can be added with a range of colours which can be scored as mild, moderate or severe. This will aid the scoring of colour.

However, not all items can be scored in a fixed manner. Complaints and clinical signs such as edema are dependent on the time of the day and on whether a patient has been highly active and is mobile or immobile. To this point we advise including items that can be easily fixed in specific outcomes. Venous claudication is one such item that should be given more attention and should be related to the impact on QOL and clinical signs. An ideal score should also be able to follow changes over time, and should be reliable, validated, and easy to use. Future research should focus on the patients' experience of complaints and impediments to daily activities. To this end, QOL should be included because it probably best reflects the impact of clinical complaints on a patients' life.

In order to address this, we initiated a randomized controlled trial to evaluate QOL before and after interventional deep venous treatment. Chapter 10 presents the research protocol.

At the present time, physicians face the difficulty of not being able to predict treatment outcome. By analysing $\mathrm{QOL}$ and comparing the non-treated and treated patients, this study aims to provide physicians with better insight into the prediction of treatment outcome.

Current literature is based mainly on single centre retrospective cohort studies in whose results cannot be compared. Available studies use also different interventional techniques which may contribute to differences in stent outcomes. For example, there is a divergence of opinion on where best to land the stent for iliac compression syndromes: some physicians prefer the iliac confluence, whereas others prefer to land the stent into the vena cava. This preference is, off course, also dependant on the stent type physicians are able to use. In literature, different types of stents have been used. In countries with strict government regulation and high out-of-pocket costs, non-venous dedicated stents are used. Outcomes cannot be compared and data cannot be pooled when these differences maintain. There is also lack of consensus in current existing literature about which preoperative imaging modalities should be used. Due to the heterogeneity in preoperative imaging protocols a selection bias can be expected as several imaging modalities show venous pathology in detail, such as IVUS, while others show rough patterns, such as CTV. The clinical importance of the two types of findings is not yet defined and it is important for future trials to help create consensus in preoperative imaging as well as the pathology that should be stented. The goal of present randomized trial is to indicate the conditions that lead to significantly better outcomes for dedicated venous stents, with the larger aim of optimising and standardising treatment plans all over the world. 
We recognize that the great heterogeneity in deep venous obstruction patterns makes challenging the comparison of all outcomes. Further, post interventional confounding factors can play a great role in the ultimate outcome. For example, a patients' mobility can play a possible role in the occurrence of stent stenosis or occlusion. Post interventional anticoagulation may also be a confounder in treatment outcomes and influence patency rates. Finally, we will not be able to analyse whether patients in the conventional treatment group use their compressive stockings on a daily basis, which could bias outcomes in this group.

Altogether, there will be some persisting issues which cannot be excluded due to the heterogeneity of subjects, but by performing this randomized clinical trial, we will show clinical outcomes after interventional treatment compared to conventional treatment in which part of known confounders are fixed in order to minimize bias. 


\section{References}

1. Guanella R, Ducruet T, Johri M, Miron MJ, Roussin A, Desmarais S, et al. Economic burden and cost determinants of deep vein thrombosis during 2 years following diagnosis: a prospective evaluation. J Thromb Haemost. 2011;9(12):2397-405.

2. Kahn SR, Shbaklo H, Lamping DL, Holcroft CA, Shrier I, Miron MJ, et al. Determinants of health-related quality of life during the 2 years following deep vein thrombosis. J Thromb Haemost. 2008;6(7): 1105-12.

3. Lubberts B, Paulino Pereira NR, Kabrhel C, Kuter DJ, DiGiovanni CW. What is the effect of venous thromboembolism and related complications on patient reported health-related quality of life? A meta-analysis. Thromb Haemost. 2016;116(3):417-31.

4. Raju S, Neglen P. High prevalence of nonthrombotic iliac vein lesions in chronic venous disease: a permissive role in pathogenicity. J Vasc Surg. 2006;44(1):136-43.

5. Wittens C, Davies AH, Baekgaard N, Broholm R, Cavezzi A, Chastanet S, et al. Editor's Choice Management of Chronic Venous Disease: Clinical Practice Guidelines of the European Society for Vascular Surgery (ESVS). Eur J Vasc Endovasc Surg. 2015;49(6):678-737.

6. Galanaud JP, Righini M, Quere I. Compression stockings to prevent post-thrombotic syndrome. Lancet (London, England). 2014;384(9938):129.

7. Mohr DN, Silverstein MD, Heit JA, Petterson TM, O'Fallon WM, Melton LJ. The venous stasis syndrome after deep venous thrombosis or pulmonary embolism: a population-based study. Mayo Clin Proc. 2000;75(12):1249-56.

8. Prandoni P, Villalta S, Bagatella P, Rossi L, Marchiori A, Piccioli A, et al. The clinical course of deep-vein thrombosis. Prospective long-term follow-up of 528 symptomatic patients. Haematologica. 1997;82(4):423-8.

9. Kahn SR, Solymoss S, Lamping DL, Abenhaim L. Long-term outcomes after deep vein thrombosis: postphlebitic syndrome and quality of life. J Gen Intern Med. 2000;15(6):425-9.

10. Prandoni P, Lensing AW, Cogo A, Cuppini S, Villalta S, Carta M, et al. The long-term clinical course of acute deep venous thrombosis. Ann Intern Med. 1996;125(1):1-7.

11. Neglen P, Thrasher TL, Raju S. Venous outflow obstruction: An underestimated contributor to chronic venous disease. J Vasc Surg. 2003;38(5):879-85.

12. Kurstjens RL, de Wolf MA, Konijn HW, Toonder IM, Nelemans PJ, de Graaf R, et al. Intravenous pressure changes in patients with postthrombotic deep venous obstruction: results using a treadmill stress test. J Thromb Haemost. 2016;14(6):1163-70.

13. AbuRahma AF, Robinson PA, Boland JP. Clinical, hemodynamic, and anatomic predictors of long-term outcome of lower extremity venovenous bypasses. J Vasc Surg. 1991;14(5):635-44.

14. Palma EC, Esperon R. Vein transplants and grafts in the surgical treatment of the postphlebitic syndrome. J Cardiovasc Surg. 1960;1:94-107.

15. Okrent D, Messersmith R, Buckman J. Transcatheter fibrinolytic therapy and angioplasty for left iliofemoral venous thrombosis. J Vasc Intervent Radiol. 1991;2(2):195-7; discussion 8-200.

16. Neglen P, Berry MA, Raju S. Endovascular surgery in the treatment of chronic primary and postthrombotic iliac vein obstruction. Eur J Vasc Endovasc Surg. 2000;20(6):560-71.

17. Arnoldussen $\mathrm{CW}$, de Graaf R, Wittens $\mathrm{CH}$, de Haan MW. Value of magnetic resonance venography and computed tomographic venography in lower extremity chronic venous disease. Phlebology. 2013;28 Suppl 1:169-75.

18. Arnoldussen CW, Wittens $\mathrm{CH}$. Assessment of the Postthrombotic Syndrome using MR Venography and DUS: The Correlation with Clinical Scoring Systems, VCSS, Villalta, and CEAP. J Vasc Surg Venous Lymphat Disord. 2013;1(1):114.

19. Blanch Alerany M, Izquierdo Lamoca LM, Ramirez Ortega M, Lago Rivas I, Zotta Desboeufs R, Stefanov Kiuri S. Endovascular treatment of iliofemoral chronic post-thrombotic venous flow obstruction. J Vasc Surg Venous Lymphat Disord. 2014;2(1):2-7. 
20. Seager MJ, Busuttil A, Dharmarajah B, Davies AH. Editor's Choice-- A Systematic Review of Endovenous Stenting in Chronic Venous Disease Secondary to Iliac Vein Obstruction. Eur J Vasc Endovasc Surg. 2016;51(1):100-20.

21. Wen-da W, Yu Z, Yue-Xin C. Stenting for chronic obstructive venous disease: A current comprehensive meta-analysis and systematic review. Phlebology. 2016;31(6):376-89.

22. Razavi MK, Jaff MR, Miller LE. Safety and Effectiveness of Stent Placement for lliofemoral Venous Outflow Obstruction: Systematic Review and Meta-Analysis. Circ Cardiovasc Intervent. 2015;8(10):e002772.

23. Comerota AJ, Oostra C, Fayad Z, Gunning W, Henke P, Luke C, et al. A histological and functional description of the tissue causing chronic postthrombotic venous obstruction. Thromb Res. 2015;135(5):882-7.

24. Spronk HMH, Padro T, Siland JE, Prochaska JH, Winters J, van der Wal AC, et al. Atherothrombosis and Thromboembolism: Position Paper from the Second Maastricht Consensus Conference on Thrombosis. Thromb Haemost. 2018;118(2):229-50.

25. Marston WA, Chinubhai A, Kao S, Kalbaugh C, Kouri A. In vivo evaluation of safety and performance of a nitinol venous stent in an ovine iliac venous model. J Vasc Surg Venous Lymphat Disord. 2016;4(1):73-9.

26. Razavi M, Marston W, Black S, Bentley D, Neglen P. The initial report on 1-year outcomes of the feasibility study of the VENITI VICI VENOUS STENT in symptomatic iliofemoral venous obstruction. J Vasc Surg Venous Lymphat Disord. 2018;6(2):192-200.

27. Caliste XA, Clark AL, Doyle AJ, Cullen JP, Gillespie DL. The incidence of contralateral iliac venous thrombosis after stenting across the iliocaval confluence in patients with acute or chronic venous outflow obstruction. J Vasc Surg Venous Lmphat Disord. 2014;2(3):253-9.

28. Duda SH, Wiskirchen J, Tepe G, Bitzer M, Kaulich TW, Stoeckel D, et al. Physical properties of endovascular stents: an experimental comparison. J Vasc Intervent Radiol. 2000;11(5):645-54.

29. Murphy EH, Johns B, Varney E, Buck W, Jayaraj A, Raju S. Deep venous thrombosis associated with caval extension of iliac stents. J Vasc Surg Venous Lymphat Disord. 2017;5(1):8-17.

30. Stuck AK, Kunz S, Baumgartner I, Kucher N. Patency and Clinical Outcomes of a Dedicated, SelfExpanding, Hybrid Oblique Stent Used in the Treatment of Common Iliac Vein Compression. J Endovasc Ther. 2017;24(1):159-66.

31. Vogel D, Comerota AJ, Al-Jabouri M, Assi Zl. Common femoral endovenectomy with iliocaval endoluminal recanalization improves symptoms and quality of life in patients with posthrombotic iliofemoral obstruction. J Vasc Surg. 2012;55(1):129-35.

32. Puggioni A, Kistner RL, Eklof B, Lurie F. Surgical disobliteration of postthrombotic deep veins-endophlebectomy--is feasible. J Vasc Surg. 2004;39(5):1048-52; discussion 52.

33. Comerota AJ, Grewal NK, Thakur S, Assi Z. Endovenectomy of the common femoral vein and intraoperative iliac vein recanalization for chronic iliofemoral venous occlusion. J Vasc Surg. 2010; 52(1):243-7.

34. Mahnken AH, Thomson K, de Haan M, O'Sullivan GJ. CIRSE standards of practice guidelines on iliocaval stenting. Cardiovasc Intervent Radiol. 2014;37(4):889-97.

35. Neglen P, Tackett TP, Jr., Raju S. Venous stenting across the inguinal ligament. J Vasc Surg. 2008; 48(5):1255-61.

36. de Wolf MA, Jalaie H, van Laanen JH, Kurstjens RL, Mensinck MJ, de Geus MJ, et al. Endophlebectomy of the common femoral vein and arteriovenous fistula creation as adjuncts to venous stenting for post-thrombotic syndrome. Br J Surg. 2017;104(6):718-25.

37. Kurstjens RL, de Graaf R, Barbati ME, de Wolf MA, van Laanen JH, Wittens $\mathrm{CH}$, et al. Arteriovenous fistula geometry in hybrid recanalisation of post-thrombotic venous obstruction. Phlebology. 2015;30(1 Suppl):42-9.

38. Williams KJ, Moore HM, Davies AH. Haemodynamic changes with the use of neuromuscular electrical stimulation compared to intermittent pneumatic compression. Phlebology. 2015;30(5):365-72.

39. Jalaie H, Arnoldussen C, Barbati M, Kurstjens R, de Graaf R, Grommes J, et al. What predicts outcome after recanalization of chronic venous obstruction: hemodynamic factors, stent geometry, patient selection, anticoagulation or other factors? Phlebology. 2014;29(1 suppl):97-103. 
40. Wittens $\mathrm{CH}$, Bukkems SF, Toonder IT. Abdominal wall venous collaterals: the latent clinical sign for central chronic venous obstruction. Circulation. 2010;122(20):2089-90.

41. Umeoka S, Koyama T, Togashi K, Kobayashi H, Akuta K. Vascular dilatation in the pelvis: identification with CT and MR imaging. Radiographics. 2004;24(1):193-208.

42. Pillai AK, Andring B, Patel A, Trimmer C, Kalva SP. Portal hypertension: a review of portosystemic collateral pathways and endovascular interventions. Clin Radiol. 2015;70(10):1047-59.

43. Evers EJ, Wuppermann T. [Ultrasound diagnosis in post-thrombotic syndrome. Comparative study with color duplex, cw-Doppler and B-image ultrasound]. Ultraschall Med. 1995;16(6):259-63.

44. Labropoulos N, Borge M, Pierce K, Pappas PJ. Criteria for defining significant central vein stenosis with duplex ultrasound. J Vasc Surg. 2007;46(1):101-7.

45. Ruehm SG, Wiesner W, Debatin JF. Pelvic and lower extremity veins: contrast-enhanced threedimensional MR venography with a dedicated vascular coil-initial experience. Radiology. 2000;215(2): 421-7.

46. Arnoldussen C, Strijkers R, Lambregts D, Lahaye M, de Graaf R, Wittens C. Feasibility of identifying deep vein thrombosis characteristics with contrast enhanced MR-Venography. Phlebology. 2014;29(1 suppl):119-24.

47. Piciucchi S, Barone D, Sanna S, Dubini A, Goodman LR, Oboldi D, et al. The azygos vein pathway: an overview from anatomical variations to pathological changes. Insights Imaging. 2014;5(5):619-28.

48. Murphy EH, Johns B, Varney E, Raju S. Endovascular management of chronic total occlusions of the inferior vena cava and iliac veins. J Vasc Surg Venous Lymphat Disord. 2017;5(1):47-59.

49. Kurstjens RL, de Wolf MA, van Laanen JH, de Haan MW, Wittens $\mathrm{CH}$, de Graaf R. Hemodynamic significance of collateral blood flow in chronic venous obstruction. Phlebology. 2015;30(1 Suppl): 27-34.

50. Kibbe MR, Ujiki M, Goodwin AL, Eskandari M, Yao J, Matsumura J. Iliac vein compression in an asymptomatic patient population. J Vasc Surg. 2004;39(5):937-43.

51. Nazzal M, El-Fedaly M, Kazan V, Qu W, Renno AW, Al-Natour M, et al. Incidence and clinical significance of iliac vein compression. Vascular. 2015;23(4):337-43.

52. Gagne PJ, Tahara RW, Fastabend CP, Dzieciuchowicz L, Marston W, Vedantham S, et al. Venography versus intravascular ultrasound for diagnosing and treating iliofemoral vein obstruction. J Vasc Surg Venous Lymphat Disord. 2017;5(5):678-87.

53. Vasquez MA, Rabe E, McLafferty RB, Shortell CK, Marston WA, Gillespie D, et al. Revision of the venous clinical severity score: venous outcomes consensus statement: special communication of the American Venous Forum Ad Hoc Outcomes Working Group. J Vasc Surg. 2010;52(5):1387-96.

54. Utne KK, Ghanima W, Foyn S, Kahn S, Sandset PM, Wik HS. Development and validation of a tool for patient reporting of symptoms and signs of the post-thrombotic syndrome. Thromb Haemost. 2016;115(2):361-7. 

Nederlandse samenvatting 



\section{Nederlandse samenvatting}

Diep veneuze obstructie is een veel voorkomende aandoening die steeds meer aandacht heeft gekregen vanwege de hoge sociaaleconomische belasting en het negatieve effect op de kwaliteit van leven (QOL) van een patiënt. ${ }^{1-3}$ De obstructie is een gevolg van chronische trabeculatie na een diep veneuze trombose (DVT) of van extrinsieke compressie, waarvan het iliacale veneuze compressiesyndroom (IVCS) het meest frequent voorkomt. ${ }^{4}$

Jarenlang was de primaire behandeling van DVT's gericht op de klinische symptomen en de preventie van complicaties op de lange termijn. Er waren slechts conservatieve behandelingsmogelijkheden. In de jaren 30 merkte Jobsts minder waarneembare symptomen op bij geïmmobiliseerde patiënten, en ontwikkelde de eerste compressiekousen. $^{5}$ In de daarop volgende veertiger jaren, daalde het aantal patiënten met chronische veneuze insufficiëntie dramatisch ten gevolge van de introductie van anticoagulantia. ${ }^{6}$ De onderliggende oorzaak van klinische symptomen bleef bij deze behandelingen nog onderbelicht.

Ondanks deze ontwikkelingen toonde onderzoek aan dat slechts bij een deel van de patiënten met iliacale DVT's de klachten volledig verdwenen. ${ }^{2,7,8}$ Patiënten ontwikkelden klachten gerelateerd aan de persisterende veneuze hypertensie, zoals pijn tijdens mobilisatie, krampen of zwaarte van de onderste extremiteiten, ook bekend als veneuze claudicatio. ${ }^{9,10}$ Deze klachten ontstonden als gevolg van posttrombotische obstructie, waaraan onvoldoende veneuze rekanalisatie ten grondslag lag. ${ }^{11,12}$ Daarnaast bestond er een tweede pathognomonische component die bijdroeg aan het persisteren van klinische symptomen, hetgeen bekend stond als veneuze incompetentie, veroorzaakt door de destructie van het klep sluitingsmechanisme.

Dientengevolge, werden interventionele technieken in hoog tempo ontwikkeld om de primaire en secundaire behandeling van DVT's te verbeteren. In de afgelopen decennia hebben de vorderingen in endovasculaire behandeling en beeldvormingstechnieken een revolutie teweeggebracht. Vóór de jaren 90, werden open bypassoperaties uitgevoerd, uitsluitend in gevallen met ernstige diep-veneuze pathologie. Deze majeure chirurgische interventies bleken geen duurzame oplossing te zijn vanwege de zwakke tot matige patency rates en gematigde klinische resultaten op middellange termijn. ${ }^{13,14}$ In de daaropvolgende jaren werden speciale veneuze beeldvormende protocollen, verbeteringen in angiografische technieken en intravasculaire echografie (IVUS) ontwikkeld. Dientengevolge, konden minimaal invasieve percutane transluminale angioplastiek (PTA) en stenting worden geïntroduceerd als vervanging van de open chirurgische reconstructies. ${ }^{15,16}$ Dit leidde tot een algehele vooruitgang in de behandeling van patiënten met diep veneuze obstructies. De endovasculaire interventies, uitgevoerd in verschillende instituten wereldwijd, leidden tot meerdere kleine case-series met goede klinische resultaten. 
Ondanks deze vroege successen werd het duidelijk dat veneuze interventies een ander benadering vereisen dan de mainstream endovasculaire arteriële interventies, door de verschillen in eigenschappen tussen venen en arteriën. Zo hebben venen een dunnere tunica media dan arteriën met als gevolg hiervan een grotere rekbaarheid. Daarnaast hebben venen kleppen die toestaan dat bloed tegen de zwaartekracht in stroomt. Er bestaan enkele andere mechanismen die deze stroom mogelijk maken, waarvan de spiercompressiepomp en de negatieve intra-thoracale druk de grootste voorbeelden zijn. Omwille van deze anatomische verschillen, lage veneuze druk en verschillen in onderliggend pathologisch mechanisme tussen veneuze en arteriële problematiek, werden speciale teams ontwikkeld om de diep veneuze pathologie in groter detail te kunnen bestuderen en om optimale behandelplannen te ontwikkelen. In de afgelopen decennia zijn, naast ontwikkelingen in interventies, ook verscheidene beeldvormingsmodaliteiten gebruikt om een diagnose te stellen en de diep veneuze pathologie beter te kunnen begrijpen. Met het vorderen van de kennis kwam men tot het inzicht dat het veneuze systeem ook anders geanalyseerd en gediagnosticeerd dient te worden dan het arteriële stelsel. Zo werd het evident dat de evaluatie van de onderste extremiteit gecombineerd moet worden met het verloop en pathologie van abdominale venen of iliacale venen, om de (frequent) verminderde functie van femorale of popliteale veneuze segmenten te kunnen begrijpen. Meerdere beeldvormingsmodaliteiten zijn in de loop der jaren de revue gepasseerd om dit te bewerkstelligen. De eerste pre-interventionele evaluatie van diepe veneuze pathologie door middel van duplex echografie (DUS) is een niet-invasieve techniek die zowel anatomische als hemodynamische inzichten in het veneuze systeem verschaft. De uitkomst van deze techniek is echter afhankelijk van de echografist, waarbij uitgebreide ervaring de grootste rol speelt. Daarnaast zijn er patiënt afhankelijke factoren waarvan obesitas, niet-samenpersbare lucht in de darm, veneuze ulceratie of een verdikte huid enkele voorbeelden zijn. De nadelige factoren binnen de DUStechniek leidde tot de ontwikkeling van veneuze cross-sectionele beeldvorming modaliteiten zoals Computer Tomografie venografie (CTV) en Magnetische resonantie venografie (MRV). ${ }^{17,18}$ CTV is in staat veneuze obstructie of veneuze compressie te identificeren en helpt bij de herkenning van typische collateralisatiepatronen. Deze techniek is overal ter wereld beschikbaar maar maakt gebruik van ioniserende straling. Bovendien blijft het een uitdaging om voldoende homogene opacificatie te verkrijgen. In de jaren 90 werd MRV gebruikt om veneuze pathologie te evalueren met als belangrijkste voordeel de mogelijkheid tot visualisatie van gedetailleerde veranderingen in de veneuze wand zoals webs, spurs en trabeculae. Daarnaast wordt er bij deze techniek geen gebruik gemaakt van ioniserende straling of jodiumhoudend contrast.

In tegenstelling tot de CTV, zijn MRI-scans echter niet overal ter wereld beschikbaar vanwege de hoge kosten die aan deze modaliteit verbonden zijn. Tevens bestaan er enkele contra-indicaties waarvan claustrofobie of patiënten met metaalfragmenten 
de meest voorkomende voorbeelden zijn. Concluderend kan men stellen dat een combinatie van echografie en MRV of echografie en CTV moeten worden gebruikt om zoveel mogelijk informatie te verschaffen voordat behandelopties kunnen worden besproken.

Met toenemende kennis en verbetering in behandelmogelijkheden voor patiënten met diepe veneuze pathologie werd in 2012 een toegewijd veneus team in Nederland opgericht en namen de diep veneuze interventies hun intrede. Onze kennis betreffende dit onderwerp, evenals de resultaten van onze zes jaar durende ervaring met endovasculaire behandeling, wordt beschreven in hoofdstuk 2.

In de beschreven studie werden drie groepen behandeld, ingedeeld op basis van de onderliggende pathologie en de omvang van post-trombotische veranderingen gedetecteerd op speciale MRV-scans en perioperatieve flebografieën. De eerste groep bestond uit patiënten met een non-thrombotische iliacale veneuze compressie die een percutane stent kregen (IVCS-groep). De tweede groep bestond uit patiënten met een post-trombotisch syndroom (PTS) die werden behandeld door middel van percutane stentplaatsing (P-PTS-groep). De laatste groep bestond uit PTS-patiënten met obstructieve veranderingen van de venen perifeer van de saphenofemorale junctie (SFJ) die een hybride procedure ondergingen, waarbij stenting wordt gecombineerd met open chirurgische desobliteratie (H-PTS groep).

Het primaire doel van stentplaatsing was het verminderen van klinische symptomen. Na 24 maanden werden daarom klinische scores geëvalueerd middels de Villalta score en Venous Clinical Severity Score (VCSS).Resultaten toonden een verbetering in Villalta score, met name bij de post-trombotische patiënten (P-PTS groep). In totaal werden 369 patiënten behandeld waarbij veneuze claudicatie in $90 \%$ van de IVCSgroep, $82 \%$ van de P-PTS groep en $83 \%$ van de H-PTS-groep verdween. Hoewel eerdere reviews niet in staat waren klinische uitkomstmaten te poolen in een metaanalyse vanwege grotere heterogeniteit, lieten sommige studies een verbetering in klinische uitkomsten zien identiek aan de resultaten gepresenteerd in onze studie. Blanch Alerany et al. toonden namelijk een significante verbetering voor posttrombotische proefpersonen in zowel de VCSS van 8 naar 2, als de Villalta-schaal van 15 naar $2(P<0,001)$, bij een follow-up van 21 maanden. ${ }^{19}$

Naast de klinische uitkomsten werden bijbehorende patency rates geanalyseerd. In onze studie resulteerde dit in een primaire doorgankelijkheid (primary patency), assisted primary patency en secundaire patency rate van $90 \%, 100 \%$ en $100 \%$ voor de IVCS-groep en 64\%, 81\% en $89 \%$ respectievelijk voor de P-PTS-groep bij een follow-up van 60 maanden. Daarentegen toonde de H-PTS-groep, een patency van respectievelijk $37 \%$, $62 \%$ en $72 \%$, bij de 36 maanden follow-up. Interessant is dat, hoewel verschillende diagnostische en interventionele technieken en stenttypen werden gebruikt, de uitkomsten van percutaan behandeld patiënten vergelijkbaar waren met eerdere studies, waarbij primaire patency tussen $32 \%$ en $98,7 \%$ en secundaire patencies van $66 \%$ tot $96 \%$ werden beschreven. ${ }^{20-22}$ 
De initiële diep veneuze behandelingen werden verricht met stents die speciaal voor het arteriële systeem ontwikkeld waren. Met het vorderen van de tijd werd het inzichtelijk dat deze stents niet optimaal waren voor gebruik in het veneuze systeem, omdat de pathologie in het veneuze systeem voornamelijk als gevolg van intraluminale veneuze verlittekening en extra luminale compressie ontstaat. Dit staat in contrast tot de athero-trombotische calcificaties die optreden in het arteriele stelsel. ${ }^{23-25}$ In het veneuze systeem is het van belang om voldoende uitwendige radiale kracht te hebben om de krachten van externe compressie tegen te gaan. Daarnaast behoeven anatomische locaties met herhaaldelijke flexie-stress, zoals de vena iliaca interna en externa, stents die compatibel zijn met dit bewegingsbereik. De stents die in de literatuur beschreven zijn en in de beginjaren van behandelde diep veneuze pathologie gebruikt werden zijn minder in staat om voldoende radiale kracht toe te staan, met als gevolg in-stent restenosering op kwetsbare anatomische locaties. De vaak gebruikte balloon-expandable Wallstent, bestaat uit gevlochten non-magnetic Cobalt-Chromium-Nickel-Molybdenum legering (Elgiloy). Dit type stent veroorzaakt een verkorting van de stent tijdens de ballon ontplooiing en is moeilijk te plaatsen bij de overgang van de vena iliaca naar de vena cava. De stent toont namelijk pas voldoende radiale kracht wanneer de uiteinden van de stent bevestigd zijn in de veneuze wand. Dit is niet het geval is bij ontplooiing ter plaatse van de confluence, omdat de stents geplaatst moeten worden zonder de veneuze instroom van de contralaterale zijde te compromitteren. ${ }^{25-27}$

Vanwege de tekortkomingen van de initieel geplaatste stents, werden speciale, zelf ontplooibare veneuze stents ontwikkeld en geïntroduceerd. Deze stents hebben een hogere flexibiliteit, kunnen hogere radiale krachten overwinnen en kunnen in lange veneuze segmenten worden geplaatst. Bovendien zijn deze stents gemaakt van nitinol, een product met thermisch vormgeheugen en superelasticiteit die rekening houdt met herstel van de stentvorm wanneer de uitwendig druk wordt verlaagd. ${ }^{(26)} \mathrm{Dit}$ in tegenstelling tot de ballonexpandeerbare stents, die onomkeerbaar worden vervormd als de externe load de ringsterkte (hoop strength) overschrijdt. ${ }^{28}$

Vormherstel van speciale veneuze stents moet bijdragen aan het verminderen van in stent-gerelateerd complicaties, zoals knikken, breken en taps toelopen (kinking, fracturing and tapering). Daarnaast hebben de ontwikkelde stents een open-cel design dat zou kunnen resulteren in een lagere contralaterale DVT incidentie. ${ }^{29}$ Dit resultaat is vooralsnog puur theoretisch ondersteund, doordat de kleinere tussenruimten van de meer rigide, gesloten-cel stents vaker met neointima bekleed lijken te zijn dan de speciale veneuze stents. Dientengevolge kunnen de gesloten-cel stents een gereduceerde veneuze doorstroming geven en als gevolg daarvan tevens de contralaterale veneuze instroom belemmeren.

Momenteel ontbreken onderzoeken die de klinische resultaten en resultaten op het gebied van open- en gesloten- cel designs vergelijken. Desalniettemin zijn er al gunstige resultaten beschreven in casussenreeksen van patiënten die behandeld 
werden met speciale veneuze stents. ${ }^{26,30}$ Stuck et al. presenteerde een $85 \%$ primaire doorgankelijkheid in post-trombotische en IVCS patiënten behandeld met een Sinus Obliquus-stent. ${ }^{30}$ Daarnaast werd een afname van 6 punten op de Villalta schaal en 3 punten op de VCSS, gevonden bij een follow up van 10 maanden.

In hoofdstuk 3 presenteren we ons onderzoek naar uitkomsten bij gebruik van de speciale Sinus Venous-stent. In een groep van 200 patiënten, nam de gemiddelde Villalta schaal af van 10,5 naar 5,3 ( $P<0,001)$ en de VCSS-score verminderde met een totaal van 3 punten $(P<0,001)$. Algehele patency rates onthulden een primaire patency van $68 \%$, een geassisteerde primaire patency van $83 \%$ en een secundaire doorgankelijkheid van 90\% met een mediane follow-up van 12 (11-12) maanden. Omdat klinische scores en korte termijn patency scores vergelijkbaar leken tussen de veneuze stents en de meer rigide stents, werd onze aandacht verschoven naar het aantal interventie-gerelateerde complicaties. Eerdere single centre cohort studies, die grotendeels retrospectief zijn, laten een hoog technisch succes ratio zien tot $98 \%$ met lage complicatie ratio's $(0-8,7 \%) .{ }^{20-22}$ Ter vergelijking, toont hoofdstuk 3 van dit proefschrift dat er 122 (61\%) patiënten werden behandeld zonder complicaties gedurende de follow-up periode. De meest voorkomende waargenomen complicaties waren in-stent restenose $(n=23)$ of occlusie $(n=24)$ van het gestente traject. Bovendien hebben we geen stent gerelateerde problemen gevonden zoals kinking, fracturing of tapering. We hebben geconstateerd dat de hybride procedures het grootst aantal complicaties veroorzaakte, hetgeen gerelateerd is aan de uitgebreidere pathologie en aanverwante interventie.

In tegenstelling tot eerdere gepubliceerde studies presenteert dit proefschrift een grote groep hybride interventies en toont aan dat het complicatierisico na een dergelijke ingreep hoog is met percentages tot $81 \%$. Daarnaast is het risico op een herinterventie met een percentage van $59 \%$ redelijk groot. Deze bevindingen komen overeen met onze verwachtingen, aangezien bevindingen uit eerdere literatuur hebben aangetoond dat de uitgebreidheid van het post thrombotische segment gerelateerd is aan de hoogte van patency rates en als gevolg hiervan de ernst van klinische uitkomsten. ${ }^{31-33}$ Als gevolg daarvan, wordt de plaatsing van veneuze stents perifeer van de sapheno-femorale junctie (SFJ) wordt dan ook niet specifiek behandeld in de huidige richtlijnen. ${ }^{34}$

Tijdens het vorderen van onze kennis en kunde werd opgemerkt dat wij een grote groep patiënten met post-trombotische veranderingen perifeer van de SFJ poliklinisch hadden geëvalueerd. Om deze reden zijn we op zoek gegaan naar een interventionele therapieën om patiënten met post trombotische obstructies, perifeer en nabij de SFJ, te verbeteren. De grootste uitdaging bestond uit het selecteren van patiënten met een niet-fibrotisch veneus segment, waarin de stent zou kunnen worden geplaatst. De wijdverspreide overtuiging dat stents moeten worden geplaatst "van gezond naar gezond" weefsel ligt hieraan ten grondslag. ${ }^{35}$ Omdat eerdere beschikbare behandelopties beperkt waren, werden sommige van onze patiënten reeds behandeld 
middels een endoflebectomie van de vena femoralis communis (CFV) gevolgd door aanleg van een arterio veneuze fistel (AVF), beter bekend als de hybride interventie. Dit was voornamelijk mogelijk bij patiënten waarbij de post-trombotische veranderingen zich uitstrekten tot de CFV of bij patiënten waarbij verwacht werd dat het stenten van de CFV intraluminaal weefsel zou verdringen over de openingen van de vena femoralis (FV) en diepe vena femoralis profunda (DFV). Na het ontplooien van de stents, werd een AVF gecreëerd om initiële hoge flow te genereren en thrombusvorming te beperken in het recent geopereerde gebied.

Aangezien opgemerkt werd dat deze hybride procedures meer vatbaar waren voor complicaties, en meer specifiek voor een stenose perifeer van de CFV, werd de geometrie van de fistels veranderd. ${ }^{36}$ Aanvankelijk dacht men dat een goede instroom in het volledig gerekanaliseerde en geëxploiteerd veneuze segment van het grootste belang was. Als gevolg hiervan werden AVF's in een rechte vorm geplaatst en perifeer vaan het endoflebectomie gebied aangelegd. Na verloop van tijd resulteerde dit in functionele lumenobstructies en patency loss ten gevolge van intimale hyperplasie. Dit leidde tot verandering van geometrie van AVF's naar een lusachtige meer craniale positie waarbij de lumendiameter groter was en minder shear stress zou optreden, met als gevolg meer behoud van functioneel lumen. ${ }^{37} \mathrm{Bij}$ het evalueren van de uitkomsten van de twee geometrie-technieken werd een onderscheid gemaakt tussen de twee groepen, craniaal geplaatste fistels in vergelijking tot de caudaal geplaatste fistels. Helaas kon er geen statistisch significant verschil in uitkomst worden gevonden maar het risico op occlusie en stent-gerelateerde complicaties was echter in het voordeel van craniaal geplaatste fistels. Als gevolg hiervan werden fistels op een lusachtige manier aangelegd en follow-up werd uitgevoerd om mogelijke korte en middellange termijn complicaties te evalueren. De ontwikkeling van potentiële perifere stenosering bleef een multifactorieel proces waarbij schade aan de veneuze wand en AVF-flow waarschijnlijk een belangrijke rol spelen. Naast deze voortschrijdende problematiek bleven de lange termijn veranderingen veroorzaakt door een AVF onbekend. Zo zouden intima-hyperplasie ${ }^{37}$ of cardiale overbelasting in de toekomst kunnen optreden. Omwille van deze mogelijke complicaties was er nog geen sprake van een optimaal behandelplan en werd verder gezocht naar een alternatieve behandelingsmodaliteit.

Aangezien de algemene hypothese bestond dat endotheliale bedekking van de stent aanwezig was in $86 \%$ van stent stuts na een periode van 56 dagen, evalueerden we in alle patiënten de veneuze flow 6 weken tot 3 maanden na de primaire procedure om te beslissen of de AVF patent moest blijven of dat de AVF geoccludeerd kon worden. Gedurende deze procedure werd de contralaterale arterie percutaan aangeprikt en via deze route een ballon geplaatst in de ipsilaterale arterie. Deze zorgde voor een tijdelijk occlusie van de arteriele lus van de AVF. Vervolgens werd een flebografie uitgevoerd met contrast toediening vanuit het veneuze stelsel in de voet. De contrast 
uitwas werd geëvalueerd en een volledige contrast uitwas binnen 4 seconden werd als voldoende beschouwd. Indien er sprake was van voldoende contrast uitspoeling, werd een definitieve AVF-occlusie met een Amplatzer-plug uitgevoerd. Bij patiënten met onvoldoende contrast uitwas werd beoordeeld of stent gerelateerde problemen de hoofdoorzaak waren van onvoldoende flow. Aangezien we geen verlies van patency als gevolg van kinking of tapering hadden geconstateerd, maar wel perifere stenosering zagen, werd geëvalueerd of stenting perifeer naar de CFV haalbaar was na een primaire hybride interventie.

Zoals beschreven in hoofdstuk 4, werd in 24 (38\%) van de 64 patiënten een additionele stenting in de FV of DFV verricht. Wanneer de veneuze instroom onvoldoende werd geacht, werd er extra stenting uitgevoerd in het segment met de meest dominante instroom, en werd de AVF-occlusie uitgesteld. De primaire, geassisteerde primaire en secundaire patency rates waren $60 \%, 70 \%$ en $70 \%$ respectievelijk. De Villalta-score werd verlaagd met 6,2 punten $(P<0,001)$ en de VCSS met 2,7 punten $(P=0,034)$ bij een mediane follow-up van 14 maanden. Deze studie toonde dat in geselecteerde gevallen perifere stenting mogelijk is als een bailout-optie met een lage complicatiegraad en goede klinische resultaten. Met deze resultaten in het achterhoofd, werd er gepostuleerd of het mogelijk zou zijn om gevallen te selecteren waarin perifere stenting primair zou kunnen worden uitgevoerd. Op deze manier kon het hoge aantal complicaties en herinterventies gereduceerd worden. Tijdens de selectie van patiënten hebben we in overweging genomen dat het verrichten van een endoflebectomie wellicht superieur is voor patiënten met uitgebreide post-trombotische pathologie in de potentiële target venen. Zo lijkt voor patiënten met uitgebreide pathologie in de FV en DFV en met een kleine lumendiameter, de hybride procedure de beste optie te zijn. Daarnaast bestaat het gevaar van primaire stenting op een hoger tromboserisico door een relatief lage veneuze druk in een langgerekt segment.

Hoofdstuk 5 beschrijft de resultaten van patiënten die werden behandeld met primaire stenting perifeer van de CFV. In totaal werden 14 proefpersonen behandeld met primaire, geassisteerde primaire en secundaire patency rates van $92 \%$ bij een mediane follow-up van 481 dagen. De VCSS nam af van een gemiddelde van 8,9 naar een gemiddelde van $6,4(P=0,03)$. De Villalta score nam af met een gemiddelde van 11,7 naar $4,3(P=0,003)$. Omwille hiervan is stenting perifeer van de femorale overgang in een dominant instroomvat, naar onze mening, een mogelijk alternatief voor een specifieke geselecteerde groep patiënten. Echter, deze resultaten mogen niet worden overschat, omdat er geen vergelijkende studies zijn uitgevoerd door andere centra. Daarnaast bestaat de mogelijkheid dat de resultaten in hoofdstuk 5 vooringenomen zijn, omdat we de resultaten alleen na deze specifieke behandeling kunnen beoordelen en niet kunnen voorspellen hoe de resultaten geweest zouden zijn na een hybride ingreep. Derhalve zijn verdere analyses met behulp van 
gerandomiseerde studies zijn vereist. Toekomstig onderzoek moet zich richten op de technische optimalisatie van de behandeling van diep veneus pathologieën perifeer van de SFJ. Op dit moment is het niet bekend welke mate van obstructie de veneuze stroming aanzienlijk beperkt. Tevens zijn er geen tests beschikbaar die een hemodynamisch significante obstructie nauwkeurig kunnen diagnosticeren. Een obstructie van meer dan $50 \%$ is gekozen als een willekeurig afkappunt omdat gunstige resultaten zijn gepresenteerd na stenting in deze groep patiënten. Beeldvormende technieken zoals speciale duplexprotocollen en MRI-protocollen moeten het mogelijk maken om stroomsnelheden op een reproduceerbare manier te meten. Met de focus op eerder genoemde algemene problemen, moet het aan de hand hiervan beter mogelijk zijn om resultaten te vergelijken. Op basis van de resultaten die in dit proefschrift worden gepresenteerd, is het aannemelijk dat de veneuze instroom een superieur speelt rol in de doorgankelijkheid van de stent, vergeleken met de lengte van de stent. Toekomstig onderzoek zou hierop kunnen focussen om de behandelstrategie te optimaliseren; Momenteel dragen patiënten compressiekousen of intermitterende pneumatische compressiekousen post-interventieel. Sommige theorieën beschrijven een techniek waarbij continue zenuwstimulatie behulpzaam zou kunnen zijn in het creëren van spiercontracties met als gevolg een constante bloedstroom door het gestente traject. Kleine case series beschrijven positieve effecten in het genereren van veneuze flow in gezonde personen. ${ }^{38}$ Al deze ideeën moet in de klinische praktijk worden uitgewerkt en getest om behandelingsplannen te optimaliseren.

Naast de inzichten die zijn opgedaan met betrekking tot technische optimalisatie, verwijst dit proefschrift naar andere factoren die bijdragen aan de behandelingsresultaten. ${ }^{39}$ In dit opzicht hebben we gezocht naar verschillende items die leiden tot klinische optimalisatie en patiënten selectie. Dit proces begon door de basisprincipes van een eerste consultatie van patiënten met hun arts te evalueren. Tijdens dit consult is een grondige anamnese en klinisch onderzoek standaard zorg en kan een enorme hoeveelheid aan kennis in diepe veneuze pathologie bieden. Om patiënten met een diepe veneuze obstructieve aandoening te kunnen behandelen, moeten artsen erkennen dat er veneuze klinische symptomen bestaan en zullen deze gerelateerd moeten worden aan veneuze pathologie. Bijvoorbeeld: tijdens het klinisch onderzoek kan specifiek gezocht worden naar een zichtbare prepubische collateraal, maar hier zal ook een de betekenis aan moeten worden erkend. Hoewel prepubische collateralen eerder geassocieerd werden met diep veneuze obstructies, was geen onderzoek dat deze mogelijke correlatie grondig heeft vastgelegd. ${ }^{40}$ Hoofdstuk 6 van dit proefschrift, verkent het preklinische verloop van deze diep-veneuze obstructieve klinische tekenen en meer specifiek de aanwezigheid van prepubische collateralen. Op basis van de resultaten van dit hoofdstuk is een verwijzing van een patiënt met prepubische collateralen naar een gespecialiseerd 
centrum voor aanvullende beeldvorming gerechtvaardigd. De aanwezigheid van prepubische collateralen hebben namelijk een positief voorspellende waarde van $93 \%$ op het aantonen van elke obstructie op of boven het niveau de inguinale regio. Een specificiteit van $86 \%$ werd gevonden, hetgeen wijst op enkele fout-negatieve resultaten; echter de sensitiviteit was $53 \%$ voor elke obstructie op het niveau van de lies of hoger. Deze resultaten kunnen enigszins gebiased zijn omdat de gegevens werden geëxtraheerd uit een populatie met klinische klachten gerelateerd aan problemen in de onderste extremiteiten, veneuze ulceratie of een eerdere diep veneuze trombose. In de algemene bevolking kan andere pathologie, in het bijzonder pathologie van de lever of pathologie van gynaecologische oorsprong, niet worden uitgesloten, en moet ook worden uitgesloten gezien de lage sensitiviteit in dit onderzoek. ${ }^{41,42}$ Het wordt aanbevolen deze collateralen niet te verwijderen tenzij diep veneuze pathologie is uitgesloten. Het wordt sterk aanbevolen om te informeren naar diep-veneuze pathologie, voornamelijk in symptomatisch patiënten, aangezien aanvullende beeldvorming niet-invasief is. Wanneer pathologische obstructies gevonden worden, moet worden geanalyseerd of interventionele behandeling een optie kan zijn.

De voorspelde uitkomsten van een interventionele behandelingen zijn afhankelijk van de ernst van de obstructie. De eerste stap is daarom om te bepalen hoe uitgebreid de obstructie is. Hiertoe moeten patiënten worden doorverwezen naar centra met gespecialiseerde kennis op diep veneuze gebied. Toegewijde veneuze duplexprotocollen, diepgaande kennis over diep veneuze pathologie en speciale aanvullende diagnostiek zijn een onmisbare spil in het behandelen van deze patiënten. Over het algemeen zal men in eerste instantie een evaluatie van het diep veneuze stelsel verrichten met behulp van een duplex-echografie. ${ }^{43}$ Deze techniek is zowel geschikt voor anatomische evaluatie als voor hemodynamische evaluatie. ${ }^{(44)} \mathrm{Er}$ zijn er echter enkele nadelen. Voor minder ervaren duplexlaboranten, kan het een uitdaging zijn om de abdominale- en iliacale venen nauwkeurig in kaart brengen. Bovendien zijn er enkele patiënt specifieke factoren, zoals obesitas, oedeem of darmgas, die een negatieve invloed kunnen hebben diagnostische nauwkeurigheid van een duplex onderzoek. Aanvullende diagnostiek kan worden gebruikt om deze factoren te verminderen: Diagnostische 3D-hulpmiddelen omvatten computertomografie venografie (CTV) en magnetisch resonantie venografie (MRV), die aanvullende informatie over diep veneuze stenoses of obstructie in de abdominale- of iliacale venen kan geven. ${ }^{18,45,46}$ Sinds de introductie van dedicated MRV-protocollen, zijn meerdere aspecten in het plannen van een behandeling verbeterd. Tijdens de analyse van de MR-scans hebben we gemerkt dat bepaalde specifieke collateralisatie patronen aanwezig waren bij post-trombotische patiënten. Er werden alternatieve drainagepaden ontdekt die het resultaat waren van rekanalisatie na een DVT. Specifiek voor obstructies in de vena cava, zijn er studies die de azygos en hemiazygos, 
gonadale, spinale of abdominale aderen als overheersende collateralen beschrijven. $^{41,47}$

Met betrekking tot endoveneuze interventionele behandeling, is de hypothese dat deze ontwikkelde collaterale venen overbodig worden wanneer de normale fysiologische route is hersteld. Eerdere studies hebben al aangetoond dat occlusies van de vena cava inferior en iliacale venen het meest uitdagend zijn om te behandelen. ${ }^{48}$ Echter, het hemodynamische belang van de collaterale pathways, en hun effecten op de doorgankelijkheid van de stent na een interventie, is nog niet eerder onderzocht. In een poging om verschillende verklaringen te vinden voor het falen van stent therapie, hebben we de collaterale venen van behandelde patiënten opnieuw geanalyseerd op basis van pre-operatieve MRV-scans. Hoofdstuk 7 heeft aangetoond dat een vena cava occlusie en de oppervlaktemetingen van de vena azygos en vena hemiazygos positief en statistisch significant gerelateerd waren (OR 1.01, 95\% Cl 1.003-1.019, $P=0.004$ en OF 1.007, 95\% Cl 1.001-1.013, $P=0.004$, respectievelijk). Een azygos oppervlaktemeting van $23(P<0,001)$ en een hemiazygosoppervlaktemeting van $40 \quad(P=0,008)$ werd als een afkappunt voor hogere occlusiepercentages gevonden. Interessant is dat men dacht dat de collaterale patronen een van de meest voorkomende en meest prominente tekenen kunnen zijn van significante obstructie in intraluminale ${ }^{49}$ en extraluminale veneuze compressie. Als gevolg van deze gedachte werd de aanwezigheid van veneuze collateralen op angiografische beeldvorming beschouwd als een behandelindicatie. Echter, een van de meest intrigerende aspecten van het veneuze compressiesyndromen, bestaat uit het feit dat de prevalentie van (significant) diepe veneuze compressie in de algemene populatie nog onbekend is. ${ }^{50,51}$ Bovendien is het in de praktijk immer een uitdaging om te voorspellen welke patiënten baat zullen hebben bij een interventionele behandeling en welke patiënten hier geen baat bij zullen hebben. Om deze redenen hebben we de radiografische tekenen van een asymptomatische groep proefpersonen onderzocht en bestudeerd; de resultaten van deze studie worden gepresenteerd in hoofdstuk 8.

Het is merkwaardig dat we een opmerkelijk hoog percentage angiografische kenmerken passende bij een veneuze obstructie van het iliacale veneuze systeem bij jonge en gezonde proefpersonen vonden. Hoofdstuk 8 beschrijft onze ontdekking van een mogelijk disbalans tussen klinische symptomen en bevindingen op beeldvormende technieken die kunnen leiden tot overbehandeling van patiënten. Opgemerkt moet worden, dat hoewel intravasculaire echografie (IVUS) een veelbelovend hulpmiddel lijkt, bij de evaluatie van subtiele intravasculaire veranderingen, dit type echografie niet werd gebruikt vanwege zijn invasiviteit. Hoewel de onlangs gepubliceerd VIDIO-trial aantoonde dat IVUS superieur is aan conventionele flebografie bij het diagnosticeren van iliacale stenotische laesies ${ }^{52}$, blijft IVUS een duur diagnostisch instrument dat niet routinematig wordt gebruikt door professionals. Bovendien, blijft de vraag bestaan of alle laesies, gevonden via IVUS, 
van klinisch belang zijn en interventionele therapie vereisen. Er is verder onderzoek nodig om te analyseren welke groepen patiënten profiteren van een interventionele behandeling.

De klinische uitkomsten en post-interventionele voordelen worden op dit moment geëvalueerd door klinisch onderzoek en follow-up van klinische scores. Hoewel we weten dat stenting een goede optie is voor veel patiënten, vertonen sommigen geen klinische verbetering of laten zelfs een verslechtering van klachten zien. Deze bevindingen zijn gepresenteerd in hoofdstuk 2. Gezien onze huidige kennis, is het moeilijk om te voorspellen bij welke patiënten een behandeling verbetering teweeg zal brengen. Dit kan gedeeltelijk worden verklaard door het feit dat een abnormale wetenschappelijke route is genomen in de afgelopen jaren. Het plaatsen van een diepe veneuze stent werd enkele jaren geleden gestart zonder specifiek inzicht in of kennis van het exacte pathofysiologische hemodynamische mechanisme. Eerdere studies hebben aangetoond dat angioplastiek en stenting veilig en effectief zijn, maar het actuele bewijs is zwak en voornamelijk gebaseerd op single-center retrospectieve studies. Grote klinische gegevens met diermodellen of drukmetingen ontbreken. Momenteel worden de resultaten van behandelde patiënten geanalyseerd en terug vertaald naar mogelijke verklaringen die teruggaan naar de basis van van diepveneuze pathologie.

Nauwkeurige en consistente diagnostisering is een belangrijke issue die uitgezocht moet zijn voordat de behandelopties besproken worden. Volgens eerdere studies komt PTS voor bij 20-50\% van de patiënten na een iliofemoral DVT. ${ }^{2,71}$ De belangrijkste redenen voor deze brede range zijn de verschillende diagnostische hulpmiddelen die werden gebruikt om PTS in deze studies te beoordelen; het tijdsinterval tussen acute DVT- en de PTS-beoordeling en het aantal poliklinische evaluaties zijn erg heterogeen in de bestaande literatuur.

Met algemene klinische verschijnselen zoals pijn en oedeem, is aangetoond dat PTS een niet-specifiek syndroom is en moeizaam onderscheiden kan worden van andere ziekten. In praktijk, wordt verondersteld dat een eerdere DVT en klachten van de onderste extremiteit, indicatief zijn voor PTS. Tot op heden is er geen enkele objectieve test die PTS kan diagnosticeren. Klinische scores zoals VCSS en Villalta worden gebruikt om longitudinale klinische symptomen te beschrijven, klinische verschijnselen te vervolgen en het effect van het plaatsen van diepe veneuze stents te evalueren. Deze tools hebben echter verscheidene voor- en nadelen. Ten eerste werd de VCSS initieel ontwikkeld om chronische veneuze ziekten te registreren en symptomen in de loop van de tijd te vervolgen. ${ }^{53}$ Het belangrijkste nadeel van dit scoringsitem is dat de hoogte van de score niet overeenkomt met de veneuze druk. Zo kunnen patiënten met een normale ambulante veneuze druk de diagnose ernstige PTS hebben op basis van het scoringssysteem. Daarnaast worden klinische symptomen enkel en alleen in rekening gebracht in één scorend item. Bovendien is het moeilijk deze score longitudinaal te vervolgen omdat huidpathologie, zoals opgenomen in de 
huidige scoring, niet zal veranderen en zijn zeer subjectief is. Over het algemeen nemen totaalscores af na interventionele behandeling, wanneer er sprake is van verlichting van oedeem of pijn of wanneer patiënten compressie kousen in mindere mate hoeven te dragen.

Ten tweede is de Villalta-schaal ontwikkeld door Villalta et al. en enkel en alleen als een abstract gepubliceerd. De Villalta-schaal toonde in eerdere onderzoeken een goede correlatie met de $\mathrm{QOL}$ en toont een uitstekende interobserver betrouwbaarheid. Sommige aspecten van de schaal zijn echter nog niet gevalideerd. Dit kan resulteren in een gebiasde interobserver betrouwbaarheid. Bovendien bevat de Villalta schaal 12 items, bestaande uit 5 items gerelateerd aan klinische klachten en 7 items met betrekking tot klinische tekenen bij lichamelijk onderzoek. Het belangrijkste nadeel van dit scoringssysteem bestaat uit het feit dat een deel van de items moeten worden gescoord door de arts en een deel van de items moet worden gescoord door de patiënt. Als gevolg hiervan moeten patiënten een arts bezoeken om een totale Villalta-score te krijgen. Om deze reden, werd de patiënt- gerapporteerde Villalta-schaal ontwikkeld, waarvan in eerdere onderzoeken is aangetoond dat ze veelbelovende resultaten hebben. Helaas is het niet geëvalueerd in combinatie met andere PTS-markers zoals gezondheid gerelateerde kwaliteit van leven (HRQOL). ${ }^{(54)} \mathrm{Om}$ tegemoet te komen aan de behoefte om het effect van de behandeling in de loop van de tijd te beoordelen, en dit te laten doen door één persoon, ontwikkelden we de Self-Assessed Venous Severity-Questionnaire (SAVS-Q). Deze vragenlijst omvat een visueel geassisteerde Villalta-schaal, VCSS-score, QoL-schaal en vragen met betrekking tot veneuze claudicatio. In deze studie, scoorden twee artsen / onderzoekers alle items; vervolgens scoorde de patiënt alle items op onafhankelijke wijze (Q1 en Q2). In totaal kwamen 64 patiënten in aanmerking voor analysering en voltooide een subgroep van 39 patiënten een tweede vragenlijst 1-7 dagen na het eerste bezoek. De door de arts gerapporteerde VCSS- score toonde een interklassecorrelatie van $0,51, P<0,01$ in vergelijking met de zelfbeoordeling van de score van de patiënt. De VCSS-betrouwbaarheid was het hoogst in de arts en getrainde onderzoeksgroep met een interklassecorrelatie van $0,75, P<0,01$. Wanneer de zelf beoordeelde Villalta-schaal van de patiënt werd vergeleken met de door de arts gerapporteerde schaal werd er een betrouwbaarheid van $\mathrm{k} 0,04$ gevonden. Een vergelijking van de scores van VEINES / QOL en klinische scores vertoonden de hoogste correlatie tussen Q1 en Q2. De veneuze claudicatio vragen vertoonden een zeer hoge correlatie met de VEINES / QOL van -0,97 $P<0,01$. Wij zijn van mening dat deze SAVS-Q kan worden gebruikt als basis voor toekomstige veneuze vragenlijsten, maar er zijn enkele beperkingen die nader moeten worden geanalyseerd. In het bijzonder, scores moeten reproduceerbaar zijn om bruikbaar te zijn voor de opvolging van behandeling. Dat wil zeggen, de subjectiviteit van scorende items moet worden geminimaliseerd door te beschrijven vaste items die bijdragen aan een bepaalde score. Hyperpigmentatie wordt bijvoorbeeld gescoord als mild, matig of ernstig. Als 
we ermee instemmen dit item in de uiteindelijke veneuze vragenlijst op te nemen, kan bijvoorbeeld een kleurenschaal worden toegevoegd met een reeks kleuren die mild, matig of ernstig aangeven. Echter, niet alle items kunnen op een vaste manier worden gescoord. Klachten en klinische symptomen zoals oedeem zijn afhankelijk van het tijdstip van de dag en van de vraag of een patiënt zeer actief of onbeweeglijk is geweest. Het advies is daarom om alleen items toe te voegen die makkelijk te specificeren zijn. Veneuze claudicatio is zo'n item dat meer aandacht moet krijgen en verband moet leggen met de impact op QOL en klinische symptomen. Een ideale score moet ook in de loop van de tijd veranderingen kunnen volgen, en moet betrouwbaar, gevalideerd en gebruiksvriendelijk zijn. Toekomstig onderzoek moet zich richten op de belemmeringen die patiënten ervaren in dagelijkse activiteiten. Daartoe, zou QOL moeten worden opgenomen in de vragenlijst omdat het waarschijnlijk de impact van klinische klachten op het leven van een patiënt het best weerspiegelt. Om dit aan te pakken, hebben we een gerandomiseerde gecontroleerde studie gestart om QOL te evalueren voor en na een interventionele diepe veneuze behandeling. Hoofdstuk 10 presenteert het onderzoeksprotocol van deze studie.

Op dit moment worden artsen geconfronteerd met het onvermogen om de uitkomst van de behandeling te kunnen voorspellen. Deze studie heeft als doel artsen meer inzicht te geven in de voorspelling van de uitkomst van de behandeling door QOL te analyseren tussen de niet-behandelde en behandelde groepen patiënten. De huidige literatuur is voornamelijk gebaseerd op retrospectieve single-center cohortstudies waarin de resultaten niet met elkaar te vergelijken zijn. Beschikbare studies gebruiken ook verschillende interventionele technieken die kunnen bijdragen aan verschillen in stentuitkomsten. Bijvoorbeeld: er is een menging van meningen over de anatomische locatie voor het landen van een stent voor iliacale compressie syndromen: sommige artsen geven de voorkeur aan de iliacale confluence, terwijl anderen het liefst in de vena cava landen. Deze voorkeur is natuurlijk ook afhankelijk van de stent typen die artsen kunnen gebruiken. In de literatuur zijn verschillende soorten stents gebruikt. In landen met strenge overheidsregulering en hoge out-of-pocket kosten, worden nietdedicated veneuze stents gebruikt. Uitkomsten kunnen niet worden vergeleken en gegevens kunnen niet worden samengevoegd als deze verschillen behouden blijven. In de huidige bestaande literatuur bestaat er tevens een gebrek aan consensus over welke preoperatieve beeldvormingsmodaliteiten gebruikt zouden moeten worden. Als gevolg van de heterogeniteit in preoperatieve beeldvormingsprotocollen kan een selectiebias optreden aangezien verschillende beeldvormingsmodaliteiten veneuze pathologie in detail weergeven, zoals IVUS, terwijl anderen ruwe patronen reproduceren, zoals CTV. Het klinische belang van de twee soorten bevindingen is nog niet gedefinieerd en het is van belang dat toekomstige studies helpen consensus in preoperatieve beeldvorming te creëren aangaande de pathologie die zou moeten worden gestent. Het doel van de huidige gerandomiseerde studie is om de condities aan te duiden die leiden tot significant betere resultaten voor dedicated veneuze 
stents, met het grotere doel om behandelplannen over de hele wereld te optimaliseren en standaardiseren. We erkennen dat de grote heterogeniteit in diep veneuze obstructiepatronen het vergelijken van uitkomsten zeer moeizaam maakt. Daarnaast bestaan er enkele factoren die na een interventie als verstorend kunnen werken en een grote rol kunnen spelen in het uiteindelijke resultaat. Als voorbeeld noemen wij de mobiliteit van patiënten die een mogelijke rol spelen bij het optreden van stent stenoses of stent occlusie. Post-interventionele antistolling kan ook een confounder zijn in het vergelijk van uitkomsten aangezien het van invloed kan zijn op de stent doorgankelijkheid. Ten slotte zullen we niet kunnen analyseren of patiënten in de conventionele behandelingsgroep hun compressiekousen op een dagelijkse basis gebruiken, hetgeen de resultaten in deze groep kan beïnvloeden.

Samenvattend zullen er enkele aanhoudende problemen zijn die niet kunnen worden uitgesloten vanwege de heterogeniteit aan casussen, door dit gerandomiseerde klinische onderzoek uit te voeren, zullen we klinische resultaten na een interventionele behandeling in vergelijking met conventionele behandeling laten zien. Hierbij is een belangrijk deel van de bekende confounders gefixeerd om vertekening te minimaliseren. 


\section{References}

1. Guanella R, Ducruet T, Johri M, Miron MJ, Roussin A, Desmarais S, et al. Economic burden and cost determinants of deep vein thrombosis during 2 years following diagnosis: a prospective evaluation. J Thromb Haemost. 2011;9(12):2397-405.

2. Kahn SR, Shbaklo H, Lamping DL, Holcroft CA, Shrier I, Miron MJ, et al. Determinants of health-related quality of life during the 2 years following deep vein thrombosis. J Thromb Haemost. 2008;6(7): 1105-12.

3. Lubberts B, Paulino Pereira NR, Kabrhel C, Kuter DJ, DiGiovanni CW. What is the effect of venous thromboembolism and related complications on patient reported health-related quality of life? A meta-analysis. Thromb Haemost. 2016;116(3):417-31.

4. Raju S, Neglen P. High prevalence of nonthrombotic iliac vein lesions in chronic venous disease: a permissive role in pathogenicity. J Vasc Surg. 2006;44(1):136-43; discussion 44.

5. Wittens C, Davies AH, Baekgaard N, Broholm R, Cavezzi A, Chastanet S, et al. Editor's Choice Management of Chronic Venous Disease: Clinical Practice Guidelines of the European Society for Vascular Surgery (ESVS). Eur J Vasc Endovasc Surg. 2015;49(6):678-737.

6. Galanaud JP, Righini M, Quere I. Compression stockings to prevent post-thrombotic syndrome. Lancet. 2014;384(9938):129.

7. Mohr DN, Silverstein MD, Heit JA, Petterson TM, O'Fallon WM, Melton LJ. The venous stasis syndrome after deep venous thrombosis or pulmonary embolism: a population-based study. Mayo Clin Proc. 2000;75(12):1249-56.

8. Prandoni P, Villalta S, Bagatella P, Rossi L, Marchiori A, Piccioli A, et al. The clinical course of deep-vein thrombosis. Prospective long-term follow-up of 528 symptomatic patients. Haematologica. 1997;82(4):423-8.

9. Neglen P, Thrasher TL, Raju S. Venous outflow obstruction: An underestimated contributor to chronic venous disease. J Vasc Surg. 2003;38(5):879-85.

10. Kurstjens RL, de Wolf MA, Konijn HW, Toonder IM, Nelemans PJ, de Graaf R, et al. Intravenous pressure changes in patients with postthrombotic deep venous obstruction: results using a treadmill stress test. J Thromb Haemost. 2016;14(6):1163-70.

11. Kahn SR, Solymoss S, Lamping DL, Abenhaim L. Long-term outcomes after deep vein thrombosis: postphlebitic syndrome and quality of life. J Gen Intern Med. 2000;15(6):425-9.

12. Prandoni $P$, Lensing AW, Cogo A, Cuppini S, Villalta S, Carta $M$, et al. The long-term clinical course of acute deep venous thrombosis. Ann Intern Med. 1996;125(1):1-7.

13. AbuRahma AF, Robinson PA, Boland JP. Clinical, hemodynamic, and anatomic predictors of long-term outcome of lower extremity venovenous bypasses. J Vasc Surg. 1991;14(5):635-44.

14. Palma EC, Esperon R. Vein transplants and grafts in the surgical treatment of the postphlebitic syndrome. J Cardiovasc Surg (Torino). 1960;1:94-107.

15. Okrent D, Messersmith R, Buckman J. Transcatheter fibrinolytic therapy and angioplasty for left iliofemoral venous thrombosis. J Vasc Interv Radiol. 1991;2(2):195-7; discussion 8-200.

16. Neglen P, Berry MA, Raju S. Endovascular surgery in the treatment of chronic primary and postthrombotic iliac vein obstruction. Eur J Vasc Endovasc Surg. 2000;20(6):560-71.

17. Arnoldussen $\mathrm{CW}$, de Graaf $\mathrm{R}$, Wittens $\mathrm{CH}$, de Haan $\mathrm{MW}$. Value of magnetic resonance venography and computed tomographic venography in lower extremity chronic venous disease. Phlebology. 2013;28 Suppl 1:169-75.

18. Arnoldussen CW, Wittens CH. Assessment of the Postthrombotic Syndrome using MR Venography and DUS: The Correlation with Clinical Scoring Systems, VCSS, Villalta, and CEAP. J Vasc Surg Venous Lymphat Disord. 2013;1(1):114.

19. Blanch Alerany M, Izquierdo Lamoca LM, Ramirez Ortega M, Lago Rivas I, Zotta Desboeufs R, Stefanov Kiuri S. Endovascular treatment of iliofemoral chronic post-thrombotic venous flow obstruction. J Vasc Surg Venous Lymphat Disord. 2014;2(1):2-7. 
20. Seager MJ, Busuttil A, Dharmarajah B, Davies AH. Editor's Choice-- A Systematic Review of Endovenous Stenting in Chronic Venous Disease Secondary to Iliac Vein Obstruction. Eur J Vasc Endovasc Surg. 2016;51(1):100-20.

21. Wen-da W, Yu Z, Yue-Xin C. Stenting for chronic obstructive venous disease: A current comprehensive meta-analysis and systematic review. Phlebology. 2016;31(6):376-89.

22. Razavi MK, Jaff MR, Miller LE. Safety and Effectiveness of Stent Placement for lliofemoral Venous Outflow Obstruction: Systematic Review and Meta-Analysis. Circ Cardiovasc Interv. 2015;8(10): e002772.

23. Comerota AJ, Oostra C, Fayad Z, Gunning W, Henke P, Luke C, et al. A histological and functional description of the tissue causing chronic postthrombotic venous obstruction. Thromb Res. 2015; 135(5):882-7.

24. Spronk HMH, Padro T, Siland JE, Prochaska JH, Winters J, van der Wal AC, et al. Atherothrombosis and Thromboembolism: Position Paper from the Second Maastricht Consensus Conference on Thrombosis. Thromb Haemost. 2018;118(2):229-50.

25. Marston WA, Chinubhai A, Kao S, Kalbaugh C, Kouri A. In vivo evaluation of safety and performance of a nitinol venous stent in an ovine iliac venous model. J Vasc Surg Venous Lymphat Disord. 2016;4(1):73-9.

26. Razavi M, Marston W, Black S, Bentley D, Neglen P. The initial report on 1-year outcomes of the feasibility study of the VENITI VICI VENOUS STENT in symptomatic iliofemoral venous obstruction. J Vasc Surg Venous Lymphat Disord. 2018;6(2):192-200.

27. Caliste XA, Clark AL, Doyle AJ, Cullen JP, Gillespie DL. The incidence of contralateral iliac venous thrombosis after stenting across the iliocaval confluence in patients with acute or chronic venous outflow obstruction. J Vasc Surg Venous Lymphat Disord. 2014;2(3):253-9.

28. Duda SH, Wiskirchen J, Tepe G, Bitzer M, Kaulich TW, Stoeckel D, et al. Physical properties of endovascular stents: an experimental comparison. J Vasc Interv Radiol. 2000;11(5):645-54.

29. Murphy EH, Johns B, Varney E, Buck W, Jayaraj A, Raju S. Deep venous thrombosis associated with caval extension of iliac stents. J Vasc Surg Venous Lymphat Disord. 2017;5(1):8-17.

30. Stuck AK, Kunz S, Baumgartner I, Kucher N. Patency and Clinical Outcomes of a Dedicated, SelfExpanding, Hybrid Oblique Stent Used in the Treatment of Common Iliac Vein Compression. J Endovasc Ther. 2017;24(1):159-66.

31. Vogel D, Comerota AJ, Al-Jabouri M, Assi Zl. Common femoral endovenectomy with iliocaval endoluminal recanalization improves symptoms and quality of life in patients with posthrombotic iliofemoral obstruction. J Vasc Surg. 2012;55(1):129-35.

32. Puggioni A, Kistner RL, Eklof B, Lurie F. Surgical disobliteration of postthrombotic deep veins-endophlebectomy--is feasible. J Vasc Surg. 2004;39(5):1048-52; discussion 52.

33. Comerota AJ, Grewal NK, Thakur S, Assi Z. Endovenectomy of the common femoral vein and intraoperative iliac vein recanalization for chronic iliofemoral venous occlusion. J Vasc Surg. 2010; 52(1):243-7.

34. Mahnken AH, Thomson K, de Haan M, O'Sullivan GJ. CIRSE standards of practice guidelines on iliocaval stenting. Cardiovasc Intervent Radiol. 2014;37(4):889-97.

35. Neglen P, Tackett TP, Jr., Raju S. Venous stenting across the inguinal ligament. J Vasc Surg. 2008;48(5):1255-61.

36. de Wolf MA, Jalaie H, van Laanen JH, Kurstjens RL, Mensinck MJ, de Geus MJ, et al. Endophlebectomy of the common femoral vein and arteriovenous fistula creation as adjuncts to venous stenting for post-thrombotic syndrome. Br J Surg. 2017;104(6):718-25.

37. Kurstjens RL, de Graaf R, Barbati ME, de Wolf MA, van Laanen JH, Wittens $\mathrm{CH}$, et al. Arteriovenous fistula geometry in hybrid recanalisation of post-thrombotic venous obstruction. Phlebology. 2015;30(1 Suppl):42-9.

38. Williams KJ, Moore HM, Davies AH. Haemodynamic changes with the use of neuromuscular electrical stimulation compared to intermittent pneumatic compression. Phlebology. 2015;30(5):365-72.

39. Jalaie H, Arnoldussen C, Barbati M, Kurstjens R, de Graaf R, Grommes J, et al. What predicts outcome after recanalization of chronic venous obstruction: hemodynamic factors, stent geometry, patient selection, anticoagulation or other factors? Phlebology. 2014;29(1 suppl):97-103. 
40. Wittens $\mathrm{CH}$, Bukkems SF, Toonder IT. Abdominal wall venous collaterals: the latent clinical sign for central chronic venous obstruction. Circulation. 2010;122(20):2089-90.

41. Umeoka S, Koyama T, Togashi K, Kobayashi H, Akuta K. Vascular dilatation in the pelvis: identification with CT and MR imaging. Radiographics. 2004;24(1):193-208.

42. Pillai AK, Andring B, Patel A, Trimmer C, Kalva SP. Portal hypertension: a review of portosystemic collateral pathways and endovascular interventions. Clin Radiol. 2015;70(10):1047-59.

43. Evers EJ, Wuppermann T. [Ultrasound diagnosis in post-thrombotic syndrome. Comparative study with color duplex, cw-Doppler and B-image ultrasound]. Ultraschall Med. 1995;16(6):259-63.

44. Labropoulos N, Borge M, Pierce K, Pappas PJ. Criteria for defining significant central vein stenosis with duplex ultrasound. J Vasc Surg. 2007;46(1):101-7.

45. Ruehm SG, Wiesner W, Debatin JF. Pelvic and lower extremity veins: contrast-enhanced threedimensional MR venography with a dedicated vascular coil-initial experience. Radiology. 2000;215(2):421-7.

46. Arnoldussen C, Strijkers R, Lambregts D, Lahaye M, de Graaf R, Wittens C. Feasibility of identifying deep vein thrombosis characteristics with contrast enhanced MR-Venography. Phlebology. 2014;29(1 suppl):119-24.

47. Piciucchi S, Barone D, Sanna S, Dubini A, Goodman LR, Oboldi D, et al. The azygos vein pathway: an overview from anatomical variations to pathological changes. Insights Imaging. 2014;5(5):619-28.

48. Murphy EH, Johns B, Varney E, Raju S. Endovascular management of chronic total occlusions of the inferior vena cava and iliac veins. J Vasc Surg Venous Lymphat Disord. 2017;5(1):47-59.

49. Kurstjens RL, de Wolf MA, van Laanen JH, de Haan MW, Wittens $\mathrm{CH}$, de Graaf R. Hemodynamic significance of collateral blood flow in chronic venous obstruction. Phlebology. 2015;30(1 Suppl): 27-34.

50. Kibbe MR, Ujiki M, Goodwin AL, Eskandari M, Yao J, Matsumura J. Iliac vein compression in an asymptomatic patient population. J Vasc Surg. 2004;39(5):937-43.

51. Nazzal M, El-Fedaly M, Kazan V, Qu W, Renno AW, Al-Natour M, et al. Incidence and clinical significance of iliac vein compression. Vascular. 2015;23(4):337-43.

52. Gagne PJ, Tahara RW, Fastabend CP, Dzieciuchowicz L, Marston W, Vedantham S, et al. Venography versus intravascular ultrasound for diagnosing and treating iliofemoral vein obstruction. J Vasc Surg Venous Lymphat Disord. 2017;5(5):678-87.

53. Vasquez MA, Rabe E, McLafferty RB, Shortell CK, Marston WA, Gillespie D, et al. Revision of the venous clinical severity score: venous outcomes consensus statement: special communication of the American Venous Forum Ad Hoc Outcomes Working Group. J Vasc Surg. 2010;52(5):1387-96.

54. Utne KK, Ghanima W, Foyn S, Kahn S, Sandset PM, Wik HS. Development and validation of a tool for patient reporting of symptoms and signs of the post-thrombotic syndrome. Thromb Haemost. 2016;115(2):361-7. 



\section{Valorisation addendum}





\section{Valorisation addendum}

\section{Socioeconomic relevance}

This thesis describes the results of treated patients with chronic deep venous obstruction. With a deep vein thrombosis incidence of 1-2 per 1000 per year and resulting $20-50 \%$ inadequate recanalization, there is an ample socioeconomic burden. Specifically, when the iliofemoral tract is involved, collateral potential is poor and complaints can be more severe. On top of that, there is a group of patients with clinical relevant iliac vein compression syndromes which should be included as a contributing factor to the socioeconomic load.

Due to the venous outflow obstruction and related debilitating complaints, patients are frequently restricted in participating the working process and are less able to join in social events. As a consequence, this creates a detrimental effect on a patients' quality of life.

Former studies did show that both medical costs and total socioeconomic costs were higher for patients who developed PTS during the follow up after a DVT. In developed countries, healthcare costs due to chronic venous obstruction are estimated at $1-2 \%$ of the national health care budget. In order to reduce this, it would be beneficial to be able to treat patients with deep venous obstructions in a highly efficient way. Former decades have made major improvements in the development of treatment options and endovascular interventions have shown promising results.

The results of interventional treated patients, presented in this thesis, provide a way to reduce clinical complaints. The reduction in venous claudication seems to be the most promising result. As a consequence, patients are able to participate in social events and can take part in the working life again.

With the refinement of diagnostic tools and improving knowledge regarding deep venous interventions it was pointed out that patient selection and individualized treatment should account for better results. As a result, current and future research should proceed and focus on less invasive ways in treating patients with deep venous obstruction and preventing major post interventional morbidity. With the introduction of the stenting technique below the sapheno-femoral junction an opening was created.

\section{Target population}

Outside the scientific community, there are various target groups involved in analyzing deep venous pathology and treatment of patients with venous obstructions. First of all, the general knowledge regarding the pathophysiologic mechanism should be brought under the attention. During decades, it was believed that venous reflux accounted for most problems after a deep venous thrombosis. With the current 
knowledge of the deep venous obstructive component, physicians should know that there are other treatment options next to the conservative management.

Moreover, it was established that in patients with lower extremity complaints the presence of abdominal or pubic collaterals should be considered as a referral indication. Specifically, this remains an important topic to reach general practitioners or dermatologists and those involved in the evaluation of patients after a deep venous thrombosis, such as doctors at the internal medicine department.

The remainder chapters in this thesis contribute to educational development of those physicians involved in the interventional treatment of patients with deep venous obstruction. First, developments and refinement of diagnostic techniques have made major contributions to the treatment one can offer nowadays. Due to the evolution of dedicated venous protocols it is possible to manufacture an individual treatment plan. As a consequence, potential percutaneous or surgical hybrid procedures can be estimated.

Although improvements have been made, there are still some persisting gaps to close. These gaps create an exceptional opportunity for medical industries to contribute to health care improvement. As we know that inflow seems to play an important role in stent patency it would be feasible to manufacture medical devices which could contribute to generate flow without the need for a surgical intervention. In the meantime there should be focus on development of analyzing system which can predict venous flow in order to predict the amount of blood flow necessary to maintain stent patency. Once this is introduced, we can inform a patient about the results that can be expected and we can further analyze which treatment strategies are cost-effective.

\section{Activities and innovation as a result of this thesis}

This thesis describes a large group of patients with extensive post thrombotic pathology. At first, hybrid procedures were performed in order to reduce clinical complaints. With refinement of techniques and while gaining more experience, we introduced percutaneous endovascular techniques. In this way, both patients and community paying health care costs can be reduced due to the lower complication rate and lesser medical costs.

With the introduction of a self-assessed score there are a few advantages in the follow up of venous patients; first of all are scorings of clinical signs and symptoms subjective and superior when provided by only one person. Second, are longitudinal scorings more reliable when performed by a single person. Due to the use of a selfassessed scoring system it is made possible to reduce the number of clinician visits and are less health care costs expected. 
Because of the start of a randomized clinical trial we will be able to show the clinical changes and more specifically the changes in quality of life after interventional treatment. Once this is realized, a tailored treatment approach can be implied. 

List of publications 



\section{List of publications}

T.M.A.J. van Vuuren, R.L.M. Kurstjens, C.H.A. Wittens, J.H.H.van Laanen, R. de Graaf: "Illusory Angiographic Signs of Significant Iliac Vein Compression in Healthy Volunteers" Eur J Vasc Endovasc Surg. 2018 Aug 29.

T.M.A.J. van Vuuren, C.H.A. Wittens, R. de Graaf: "Stent Extension Below the Common Femoral Vein in extensive chronic iliofemoral venous obstructions" J Vasc Interv Radiol. 2018 Aug;29(8):1142-1147.

T.M.A.J. van Vuuren*, S Doganci*, I.M. Toonder, R. de Graaf, C.H. Wittens: "Venous stent patency may be affected by collateral vein lumen size" Phlebology. 2018 Jan 1:268355518755959.

M.E. Barbati, A. Gombert, I. Toonder, T.M.A.J. van Vuuren, K. Schleimer, J. Grommes, C.H. Wittens, H. Jalaie: "Detecting stent geometry changes after venous recanalization using duplex ultrasound" Phlebology. 2018 Jan 1:268355518757240.

T.M.A.J. van Vuuren, S Doganci, C.H.A. Wittens: "Patency rates and clinical outcomes in a cohort of 200 patients treated with a dedicated venous stent" J Vasc Surg Venous Lymphat Disord. 2018 Jan 29.

T.M.A.J. van Vuuren, R.L. Kurstjens, M.A. de Wolf, J.H. van Laanen, C.H. Wittens, R. de Graaf: "Stent extension into a single inflow vessel is a valuable option after endophlebectomy" Phlebology. 2017 Jan 1:268355517739766.

T.M.A.J. van Vuuren, J.H.H. van Laanen, M.J.de Geus, P. Nelemans, R. de Graaf, C.H.A. Wittens: "A randomized controlled trial comparing venous stenting with conservative treatment in patients with Deep Venous Obstruction: research protocol" BMJ Open. 2017 Sep 11;7(9).

T.M.A.J. van Vuuren*, M.A.F. de Wolf*, C.W.K.P Arnoldussen, R.L.M. Kurstjens, J.H.H. van Laanen, H.Jalaie, R. de Graaf, C.H.A. Wittens: Editor's choice: "Reconstruction of the femoro-ilio-caval outflow by percutaneous and hybrid interventions in symptomatic deep venous obstruction" Eur J Vasc Endovasc Surg. 2017 Oct;54(4):495503.

T.M.A.J. van Vuuren, R.L.M. Kurstjens, C. Van Zandvoort, R. De Graaf, C.H.A. Wittens, J.H.H. Van Laanen: "Endovascular treatment of a reno-caval arteriovenous fistula induced Inferior Vena Cava aneurysm" Ann Vasc Surg. 2017 Nov;45:269. 
T.M.A.J. van Vuuren, C. Van Zandvoort, S. Doganci, R.L.M. Kurstjens, I. Zwiers, A. TenCate, C.H.A. Wittens: "Prediction of venous wound healing with Laser Speckle imaging" Phlebology. 2017 Dec;32(10):658-664.

R.L.M. Kurstjens, T.M.A.J. van Vuuren, M.A.F. de Wolf, R. de Graaf, C.W.K.P. Arnoldussen, C.H.A. Wittens: "Abdominal and pubic collateral veins as indicators of deep vein obstruction: an observational study" J Vasc Surg Venous Lymphat Disord. Oct 2016;4(4):426-33.

J.A. Wegdam, C.S. Andeweg, T.M.A.J. van Vuuren, T.S.D.V. Reilingh, H.J.V. ZaagLoonen, H. van Goor: "Disease Course of Right- and Left-sided Diverticulitis in a Western Population" J Gastrointest Dig Syst. April 2016; 6:421.

T.M.A.J. van Vuuren, E.R.M. van Haaren, Th.J. vd Kar, J.W. Kortleve, C.I.E. Scheeren: "Patient satisfaction and complication rate after mastectomy with immediate twostage breast reconstruction as compared to mastectomy without immediate breast reconstruction" Surgical practice. Aug 2015; 120-127 (19).

T.M.A.J. van Vuuren, J.B. Sintenie, M. Guijt. Casus: "presentatie na hondenbeet" Medisch contact rubriek wat ziet u?/Gezien. Nr. 12 - 19 maart 2014.

T.M.A.J. van Vuuren, J.A. Wegdam, M.C.A.Wegdam-Blans: "Darmperforatie door Salmonella typhimurium" Ned Tijdschr Geneeskd. 2013;157(24):A6161. 
Dankwoord 



\section{Dankwoord}

Promoveren doe je niet alleen.... Samenwerken is de sleutel tot succes.

Tal volle mensen hebben, direct of indirect, een waardevolle bijdrage geleverd aan de totstandkoming van dit proefschrift. Een aantal van hen wil ik graag in het bijzonder bedanken. Aangezien ik mij besef dat dit deel van mijn proefschrift wellicht het meest gelezen zal worden heb ik hier uiterste zorg aan besteed.

Prof. dr. Wittens, Beste Cees, daar staan we dan. Met trots kunnen wij zeggen dat ik de eerste vrouw ben in jouw reeks van promovendi. Ik wil je bedanken voor het vertrouwen en voor het feit dat je mij deze kans hebt gegeven. Niet alleen in mijn ontwikkeling als promovendus en arts heb jij een bijdrage geleverd maar ook bij het verkrijgen van mijn nieuwe baan heb jij een grote rol gespeeld. Buiten de discussies en vergaderingen op werkgebied ben ik blij deel uitgemaakt te hebben van het veneuze team. Regelmatig heb jij ons ook uitgenodigd voor een borrel of etentje hetgeen ons team alleen maar hechter heeft gemaakt.

Dr. de Graaf, beste Rick, ook al hebben wij regelmatig enige discussie gehad over mijn schrijfstijl in vergelijk met die van jouw, het is toch gelukt om er een boek van te maken! Bedankt voor al je ideeën, tips en adviezen en het begeleiden van mij in mijn promotietraject. Met veel plezier heb ik mijn onderzoeken samen met jouw verricht en in het bijzonder onze ballonproef procedures mee kunnen doen in de avonduren of zaterdagen. Ik wens je enorm veel succes in Deutschland en wie weet komen we elkaar in de toekomst nog tegen.

De beoordelingscommissie bestaande uit prof. dr. Steylen, prof. dr. De Haan, prof. dr. Wisselink, dr. Rennenberg en dr. Wiersema wil ik bedanken voor hun tijd en het beoordelen van dit proefschrift.

Lieve Jorinde, jij bent degene die mij introduceerde bij Cees en mij voorstelde aan het zogeheten veneuze team. Als assistent binnen het vaatteam hadden wij al veel samen gewerkt maar hierna werd dat nog intensiever. Ik bewonder je manier van werken en het feit dat je altijd klaar staat voor patiënten en collegae. Ik hoop dat voor jouw studies binnen korte tijd alle inclusies voltooid zijn zodat jouw promotie de volgende gaat zijn!

Carina, onmisbaar in het veneuze team. Bedankt voor de gezellige samenwerking, kopjes koffie, ulcusspreekuren en ons wekelijks klaaguurtje. Ik ben blij dat jij een vaste spil was in het veneuze team want stel je voor dat ik al die tijd geen lunchmaatje had gehad! Ik wens je veel geluk voor de toekomst en succes met het afronden van jouw promotietraject. 
Dear Suat, I would like to thank you for helping me with all the articles we wrote together. I liked the discussions we had together which eventually led to a few publications in the short period we worked as real partners. I wish you and your family all the best and maybe we will meet again in future work or conferences.

Ineke, dank voor jouw prettige samenwerking en ontzettende hulp bij mijn eerste statistiekopdrachten. Uiteindelijke kwamen we er samen wel uit (met name door jouw aantekeningen). Dank voor de koffiemomentjes en het luisterend oor dat jij bood als ik met een of andere issue zat. Ik ben blij dat je nu eindelijk ontzettend geniet van je vrije tijd en hoop dat we elkaar hier en daar nog wel eens tegen gaan komen.

Irwin, in beginsel leek het er op dat jij minder betrokken zou zijn bij mijn onderzoeken maar niets bleek minder waar. Door de discussies die wij samen met Suat hadden kwam er altijd weer een nieuw idee naar boven. Dank voor je bijdrage, expertise en kritische blik bij het schrijven van mijn stukken. Ik kijk uit naar jouw boekje en ideeën die daar in verwerkt zijn.

Dames van het oude CAVA-team Irma en Margriet, de gezelligheid in onze oude MDO kamer was niet hetzelfde als jullie een dagje vrij waren. Ook met jullie heb ik de menige kopjes koffie genuttigd! Margriet bedankt voor je kennis en kunde en adviezen die jij mij hebt gegeven bij het opzetten van onze veneuze studies. Tegen naderende eind van mijn carrière als veneuze PhD'er is ook jouw nieuwe collega, Chantal, zeer betrokken geweest in het rollende houden van onze RCT!

Heren van het veneuze team; leden van de Wittens boyband. Zo werden jullie nog altijd gezien toen ik mijn intrede nam in het team. Mark, dank dat jij als voorganger een deel van de studies opgezet hebt en een weg voor mij hebt vrijgemaakt. Toen ik jouw boekje zag was duidelijk dat er overeenkomsten in manier van denken zaten. Ik wens je veel succes met het afronden van je opleiding tot radioloog.

Ralph, onze samenwerking was kort maar krachtig! Ik ben blij dat jij nog achterbleef als enige lid van de boyband. Bedankt voor de fijne samenwerking en ik wens je heel veel succes met de start van je opleiding.

Rob, initieel leek het er op dat wij niet samen zouden gaan werken maar daar kwam vlot verandering in. $\mathrm{lk}$ wil je bedanken voor het mede verzamelen van mijn studiedata en wens je ontzettend veel succes in de toekomst!

Fabio en Yee lai, met jullie heb ik op onderzoeksgebied minder te maken gehad maar tijdens congressen en besprekingen werd er altijd voor de nodige onrust gezorgd. Ik 
wens jullie beiden succes bij het afronden van jullie proefschrift en jullie toekomstige loopbaan.

Pascale, eindelijk een vrouw die toegevoegd werd na enkele eenzame maanden op mijn kamer! Wat was ik blij dat jij mijn nieuwe collega werd en dat we dat voorlopig nog blijven! Mede dankzij jou ben ik nu waar ik ben en daar kan ik je niet genoeg voor bedanken. Je hebt bewezen volhardend te zijn en het gaat je lukken om je boekje in een korte tijd klaar te maken! Ik hoop dat we in de toekomst collega's blijven binnen de plastische chirurgie en wens je veel geluk en succes voor de komende maanden.

En dan mag de rest van het veneuze team uiteraard niet ontbreken. Jolanda, Carsten, Wijnand, Sandra en studenten, Simone, Britt en Philip aan jullie ook veel dank voor de hulp tijdens mijn researchperiode.

Ik wil er mijn vingers niet aan branden om diegenen die ik niet persoonlijk genoemd heb te vergeten en uit daarom mijn dank aan alle co-auteurs en andere mensen die hebben bijgedragen aan het ontstaan van mijn artikelen. In het bijzonder Fred und meine Deutsche Kollegen mochte ich gerne danken: Houman, Mohammed und Alexander.

Gabrielle, jij nam de taak als secretaresse over in een drukke periode. Jouw interesse en betrokkenheid bij de patiëntenzorg maakte dat het op vele momenten een zorg (en vele telefoontjes) minder was voor ons allen! Stella en Magda, de gezelligheid op de kamer van de 3 musketiers ontbrak nooit! Dat is dan ook de reden dat ik langs jullie kamer liep om mezelf hierna weer te motiveren verder te gaan met het bijhouden van een database of schrijven van een artikel.

Daarnaast wil ik alle medewerkers van de vaatkamer, de chirurgische afdelingen en de operatiekamer danken voor hun hulp en bijdrage, met name tijdens de weekenddagen en vrijdagmiddagen waarop wij, zonder enig probleem, onze studies konden uitvoeren. Babs, ook jouw naam mag niet ontbreken in dit rijtje aangezien de fantastische titel van dit boek mede door jou tot stand gekomen is!

Uiteraard was het niet mogelijk geweest om deze klinische studies uit te voeren zonder hulp van de mensen die dagelijks met de klinische zorg bezig zijn. Daarom zou ik graag alle chirurgen en assistenten van het MUMC bedanken voor de prettige samenwerking en begeleiding tijdens mijn klinische periode maar vooral ook tijdens mijn research periode. Dank voor jullie bijdrage en hulp bij het uitvoeren van onze onderzoeken. Ik ben blij dat ik mijn onderzoeksperiode mocht afsluiten met een fantastische WBS 2018! 
Daarnaast wil ik een woord richten aan de maatschap chirurgie van het Elkerliek ziekenhuis in Helmond aangezien ik daar geleerd heb wat het dokter zijn eigenlijk inhoud. Na enkele jaren ben ik dan ook terug gegaan naar dit ziekenhuis voor het opdoen van klinische ervaring binnen de plastische chirurgie. Ik wil de plastische chirurgen en PA bedanken voor het hartelijke ontvangst en de periode dat ik de fijne greepjes van dit prachtige vak heb mogen ervaren en leren.

Deze klinische periode zet ik nu voort in het Canisius Ziekenhuis Nijmegen. Dr. Schreinemachers, Dr. Heine, Dr. Fabre en dr. Wynberg en collega Inge dank voor jullie vertrouwen en alles wat ik in deze korte periode al geleerd heb bij jullie! Het blijft een afstand iedere dag maar de hartelijke ontvangst en gezelligheid op de polikliniek, OK en behandelcentrum maken het allemaal waard!

Zonder ontspanning in drukke tijden lukt het niet om dit werk tot een succesvol einde te brengen. Daarom, mijn allerliefste vriendjes en vriendinnetjes. Sommigen van jullie ken ik al jaren, anderen zijn later bijgesloten, maar jullie zijn allen even belangrijk.

Jullie kennen het allemaal.... Stundje op stap, dit, dat, zus, zo.....

Kennie en Bart, jullie ken ik het langst van iedereen. Timmehhh en Kenny dat was het vroeger, voor iedereen die South Park fan was. Schuimparty's in de Pepp, kamperen in Valkenburg en in een later stadium vrijgezellenfeest, bruiloft en babyshower! Ik hoop dat we nog lang onze vriendschap mogen voortzetten en kunnen genieten van jullie Bente!

Dank aan alle vriendjes voor de etentjes, filmavonden, winterbbqs, weekendjes weg, mooie carnavalsavonturen, vakanties, drankjes en dansjes die we samen hebben gedaan. Soms (en de laatste tijd wat vaker) kwam er een traantje aan te pas maar dat had dan ook altijd een goede reden. Voor geen goud had ik alle party's met jullie willen missen. Ik hoop dat we nog veel festivalletjes gaan bezoeken en nieuwe kampeeravonturen gaan beleven. Jullie zijn allen een onvoorwaardelijke steun geweest voor mij!

Jen en Nil, the married couple, wat een bijzonder moment was dat. Alle voorbereidingen zijn het waard geweest! Jeffie thanks voor je mooie verhalen over onze gedeelde passie en interesse, we gaan er vast nog veel meemaken!

Patrick en Lot, ge-wel-dig dat wij al die mooie feestjes in jullie huis mochten vieren! Ik wens jullie alle succes toe met jullie winkel en ben super benieuwd naar het eindresultaat van de verbouwing in jullie huis! 
Yenni, Mayen, bedankt voor de hilarious momentjes. Lekker dicht bij ons in de buurt! Ik wens je alle liefde toe voor de toekomst! OK DOEI

Ing, lieve schat, bedankt voor jouw gezelligheid en op zijn tijd een serieus gesprek. Ik zal je spraakberichten voor altijd onthouden (en bewaren...)!

Sven $\mathrm{H}$ en Gladys, buurtjes van de kling. Good old chillings hebben we gehad bij jullie tot over de $100 \%$ bechilling! Op de kling zetten we die tradities uiteraard voort met een stundje hottub of een avondje bankhangen!

Jannie met je aanstekelijke lach die niemand kan ontgaan. Lieve Kim ook onze zumbauurtjes (ook al hebben we die niet heel lang volgehouden) hebben bijgedragen aan ontspanning!

Ian en Kyle, my lovely mannen, super leuk dat jullie de beste feestjes van de partij zijn. Altijd wordt er gelachen om het een of (om iemand) ander(s)........

De mannen Sven G, Levi, Tom, Keoma, altijd van de partij op de juiste momenten! Geen feestelijk moment wordt gemist. Ik moet bekennen dat jullie dance-moves toch de beste zijn.

Lovely gurlsss Domi, Mieka, Mandy, Anouk en Renee, thanks voor al jullie knuffels en de goede (soms ook minder goede) roseetjes die wij samen genuttigd hebben. Ook al is het soms moeilijk om een gezamenlijke date te plannen, ik heb genoten van de dates die wij gehad hebben lieffies.

Buurvrouw Lois een speciale dank voor alle lieve woorden en kaartjes als het even tegen zat. Perry, aan jou moet ik in het kader van dit project een apart woord richten aangezien ik altijd binnen no-time bij je terecht kon als ik weer eens een photo-shop issue had. Zonder jou waren de plaatjes zeker niet zo mooi geworden.

Makaaaatje en Manuel, wat is een stapavond zonder jullie. Tot het bittere eind wordt volgehouden! Bedankt voor de lieve kaartjes en appjes als ik ze nodig had. We gaan nog vele mooie momenten beleven want ook $30+$ ers kunnen dat!

Timmie en Noeka, ouwe partygangers, topkoppel! Een drankje met jullie Yenni, Maka of Levi moet altijd goed over nagedacht worden, want je kent het wel.... Er zijn van die mensen waarbij je op sommige dingen niet kunt vertrouwen...het houdt namelijk zeer ernstig vrij zelden al op bij eentje. Maar dat is geen probleem dan kunnen we namelijk weer mooie foto's maken van de allergaafste gadgets die Noeka op de kop tikt! Dank voor alle afleiding en fantastische kaartjes en het feit dat jullie altijd klaarstaan. 
Mijn hele familie, oma's, ooms en tantes, neefjes, nichtjes en alle aanhang maar in het bijzonder mijn schoonfamilie/schwieger familie wil ik danken voor de afleiding en gezelligheid tijdens etentjes, familiedagen en uitstapjes. Ik kon altijd bij jullie terecht en jullie hebben mij in alles gesteund. Nooit werd er geklaagd of een opmerking gemaakt als ik later aansloot. Opa en oma, mijn hele Brazilië avontuur heb ik toch echt aan jullie te danken. Wat een supergave ervaring was dat!

Dan mijn Paranimfen...

Martine, Mart, mijn mattie. Ik ken je eigenlijk pas een aantal jaren maar wij begrijpen elkaar. Ik ben dan ook blij dat ik deze dag mag vieren met jou als paranimf aan mijn zijde. Vele memorabele stapavonden, skireizen, solar avonturen en de vrijdagavond carnaval kunnen allemaal in de boeken, ze waren stuk voor stuk goud waard en we gingen tot het gaatje. Ik wens jou een fantastische toekomst toe als chirurg I.O. (die mag met dikke vette hoofdletters!), hoop dat we nog lang vriendinnen zijn en dat jouw boekje binnen enkele maanden ook klaar is voor de drukker!

Arnee en Mark, lieve sis en schoonbroertje, en de kleine Loewie natuurlijk. Dank dat jullie ook altijd een luisterend oor hebben geboden en voor plezier en ontspanning zorgden in de vrije uurtjes. Ik vind het bijzonder dat jullie zo makkelijk aansluiting zochten tijdens de gezellige carnavalsuurtjes en kampeerterrein in Roermond, ondanks dat het weer (en gesleep met de spullen) niet altijd even mee zat. Arnee, super trots bin ich dats doe mien zuske bis. Ik ben blij dat we niet alleen op persoonlijk vlak maar ook werkvlak gebeurtenissen met elkaar kunnen delen en bespreken. Het feit dat jij vandaag als paranimf aan mijn zijde staat maakt dit nog mooier.

Pap en mam, en Bixie dankjewel voor alle mogelijkheden en vrijheid die jullie mij altijd hebben geboden. Niets was te veel en altijd kon ik op jullie rekenen. Ik ben dankbaar voor zo een lieve schatten als jullie. Jullie rol in de totstandkoming van dit promotieboekje is misschien niet op het eerste gezicht zichtbaar maar ik ben de persoon geworden die ik nu ben door jullie opvoeding en steun. Lieve mama altijd een hart onder de riem als ik het nodig had en uiterst bijzonder is het dat mijn cover design door jou is gemaakt pap. Ik ben trots op jullie als ouders en wij zullen ook altijd klaarstaan voor jullie.

Lieve Jor, mijn steun en toeverlaat, mijn mattie en hubbie. Jij verdient het eindwoord in dit alles want zonder jou was het nooit gelukt. Ik wil je danken voor je liefde, steunende woorden, verassingen als ik het nodig had en het feit dat je nooit geklaagd hebt of ook maar enige tegenspraak hebt gegeven als ik iets werk gerelateerd moest regelen. Altijd kon ik bij je terecht, op persoonlijk vlak maar ook als ik stukken had die ik graag door iemand wilde laten lezen en vooral om excel foefjes toe te kunnen passen. Want, mijn god, dat heeft mij ontiegelijk veel tijd bespaard! In die zin ben jij 
eigenlijk mede-auteur van alle stukken in dit boek. Al meer dan 13 jaar zijn we soulmates, delen we lief en leed en hebben de afgelopen jaren veel meegemaakt. We gaan samen een mooie toekomst tegemoet want alles is mooier met jou om mij heen. Lieverd, ik hou van jou! 



\section{Curriculum Vitae}





\section{Curriculum Vitae}

Timme M.A.J. van Vuuren was born on July $14^{\text {th }}, 1988$ in Nuth, The Netherlands. In 2006 she graduated from the high school Sint Jans college in Hoensbroek. Thereafter she attended medical school at the Maastricht University and obtained her medical degree in June 2012. As a medical student she went abroad to Teofilo Otoni, Minas Gerais, Brazil for an international internship. During her senior year she performed a clinical elective at the Department of Surgery of the Atrium Medical Center in Heerlen. Furthermore she followed a research elective at this department in collaboration with the Plastic and Reconstructive Surgery department of the Atrium Medical Center.

After medical school she worked as a resident not in training (ANIOS) at the Department of Surgery of the Elkerliek hospital in Helmond. Subsequent she worked as a senior house officer at the Department of Surgery of the Maastricht University Medical Center and started her career as a PhD-student under the supervision of prof. dr. C.H.A. Wittens and dr. R. de Graaf at the CARIM school of Cardiovascular Diseases in Maastricht. The work presented here was the basis of the current thesis and was presented at several national and international conferences.

In march 2018 she started as a resident not in training (ANIOS) at the department of Plastic and Reconstructive surgery In the Canisius Wilhemina Hospital in Nijmegen. 
